\title{
Spectrally-Resolved Light Absorption Properties of Cooled Soot From a Methane Flame
}

\author{
ADAM CODERRE \\ A thesis submitted to \\ The Faculty of Graduate Studies and Research \\ in partial fulfillment of the degree requirements of \\ MASTer of Applied SCIENCE in MEChaniCAL ENGineERING \\ Ottawa-Carleton Institute for \\ Mechanical and Aerospace Engineering \\ Department of Mechanical and Aerospace Engineering \\ Carleton University \\ Ottawa, Ontario, Canada
}

May 2009

Copyright (C) 2009 - Adam Coderre 
Library and

Archives Canada

Published Heritage

Branch

395 Wellington Street

Ottawa ON K1A 0N4

Canada
Bibliothèque et

Archives Canada

Direction du

Patrimoine de l'édition

395 , rue Wellington

Ottawa ON K1A 0N4

Canada

Your file Votre référence

ISBN: 978-0-494-52034-5

Our file Notre référence

ISBN: 978-0-494-52034-5

NOTICE:

The author has granted a nonexclusive license allowing Library and Archives Canada to reproduce, publish, archive, preserve, conserve, communicate to the public by telecommunication or on the Internet, loan, distribute and sell theses worldwide, for commercial or noncommercial purposes, in microform, paper, electronic and/or any other formats.

The author retains copyright ownership and moral rights in this thesis. Neither the thesis nor substantial extracts from it may be printed or otherwise reproduced without the author's permission.
AVIS:

L'auteur a accordé une licence non exclusive permettant à la Bibliothèque et Archives Canada de reproduire, publier, archiver, sauvegarder, conserver, transmettre au public par télécommunication ou par l'Internet, prêter, distribuer et vendre des thèses partout dans le monde, à des fins commerciales ou autres, sur support microforme, papier, électronique et/ou autres formats.

L'auteur conserve la propriété du droit d'auteur et des droits moraux qui protège cette thèse. $\mathrm{Ni}$ la thèse ni des extraits substantiels de celle-ci ne doivent être imprimés ou autrement reproduits sans son autorisation.
In compliance with the Canadian

Privacy A'ct some supporting forms may have been removed from this thesis.

While these forms may be included in the document page count, their removal does not represent any loss of content from the thesis.
Conformément à la loi canadienne sur la protection de la vie privée, quelques formulaires secondaires ont été enlevés de cette thèse.

Bien que ces formulaires aient inclus dans la pagination, il n'y aura aucun contenu manquant.

\section{Canadä}




\section{Abstract}

The optical (absorptive) properties of combustion-generated soot, crucial information for quantitative soot emission diagnostics and for climate modelling, have been determined for the particular case of cooled soot from a methane flame. Measurements were performed over a wavelength range of $450-950 \mathrm{~nm}$, by calibrating optical extinction measurements with traditional extractive methods in conjunction with a detailed uncertainty analysis. An inverted-flame burner was developed as a repeatable soot source, and extinction measurements were obtained by spectral line-of-sight attenuation. The absorption component of the total measured extinction was isolated by calculating the expected scattering contribution, according to the Rayleigh-DebyeGans approximation for polydisperse fractal aggregates, based on soot morphological information obtained by extractive sampling and electron microscopy. The actual soot volume fraction was obtained by gravimetric analysis. Results were in good agreement with several previous studies, indicating that fuel type and cooling history have little effect on the resulting optical properties of soot. A consistent trend of non-linear variation of the soot absorption function $E(m)$ with wavelength was observed. 


\section{Acknowledgements}

Hearty thanks go out to all those who have helped me get this far. Thanks to Greg Smallwood and the National Research Council of Canada for the access to facilities and resources. To the NRC combustion technical staff (Reg, Faz, both Dans, Bob, and Roland), thanks for the help setting up optical experiments, taking LII data, and generally teaching me the ropes. Special thanks go to my NRC supervisor, Kevin Thomson, for mentoring me during my stay, and to Dave Snelling, who provided guidance and helped me wrap my head around some of the tougher concepts I encountered.

Professor Johnson, I couldn't have asked for a better supervisor. Thank you for your patience, for your encouragement, and for your tolerance! Your optimism and energy are contagious. I thank you for motivating me and pushing me to go the extra mile, and for providing me the many opportunities that you have.

Thanks to my fellow students, particularly my office-mates Patrizio and Chen, for your friendship and conversation over the years. You made my time here that much more enjoyable.

Lastly, I could not have done this without the love and support of my family. To my wife Jenn, I cannot thank you enough for supporting me in this and all of my other efforts. You're the best. 


\section{Contents}

1 Introduction 1

1.1 Soot Morphology . . . . . . . . . . . . . . . 2

1.1.1 Soot Particle Agglomeration . . . . . . . . . . . . . 4

1.1.2 Aggregate Size Probability Distributions . . . . . . . . . 5

1.2 Methods of Soot Emission Measurement . . . . . . . . . . . 7

1.2.1 Gravimetric Analysis . . . . . . . . . . . 8

1.2.2 Electron Microscopy . . . . . . . . . . . . . . 9

1.2.3 Laser-Induced Incandescence . . . . . . . . . . . . . . . . 10

1.2.3.1 Effects of Laser Fluence . . . . . . . . . . . . 12

1.2.4 Line of Sight Attenuation (LOSA) . . . . . . . . . . . 13

1.3 Current Knowledge of Soot Optical Properties . . . . . . . . . . . 14

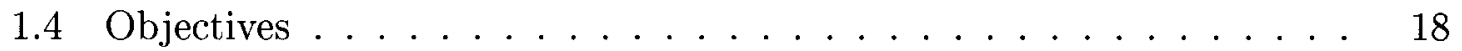

2 Light-Particle Interactions 22

2.1 Scattering and Absorption of Light by Particles . . . . . . . . . . . 22

2.2 Rayleigh Theory . . . . . . . . . . . . . . . . . 24

2.3 Mie Theory . . . . . . . . . . . . . . . . 25

2.4 RDG-PFA Approximation . . . . . . . . . . . . . 27

2.4.1 Scattering Regimes and the Structure Factor . . . . . . . . . 29 
2.4.2 Explicit Form of the Structure Factor . . . . . . . . . . . 32

2.4.3 Polydisperse Populations and Probability Distributions . . . . 33

2.4 .4 Scatter-to-Absorption Ratio . . . . . . . . . . . . . . . 35

2.4.5 Evaluation of RDG-PFA Theory . . . . . . . . . . . 36

3 Experimental Setup $\quad 38$

3.1 Inverted Flame Soot Generator . . . . . . . . . . . . . . . 39

3.1 .1 Burner Conditions . . . . . . . . . . . . . . . . 43

3.2 Gravimetric Analysis . . . . . . . . . . . . . . . . . 43

3.2 .1 Isokinetic Sampling System $\ldots \ldots \ldots \ldots$. . . . . . . 46

3.3 Soot Characterization . . . . . . . . . . . . . . 47

3.3 .1 TEM sampling $\ldots \ldots \ldots \ldots \ldots \ldots \ldots$

$3.3 .2 \quad$ SEM sampling . . . . . . . . . . . . . . . . . . 48

3.3 .3 SEM Image Analysis . . . . . . . . . . . . . . . . . 50

3.3.4 Soot Aggregate Characterization $\ldots \ldots \ldots \ldots$

3.3.5 Fitting Data to Probability Distributions . . . . . . . . 57

3.4 Numerical Calculation of $\rho_{s a} \ldots \ldots \ldots \ldots \ldots \ldots$

3.5 Spectral Line of Sight Attenuation . . . . . . . . . . . . 62

3.5 .1 Spec-LOSA Analysis . . . . . . . . . . . . . . . 65

3.6 Laser-Induced Incandescence (LII) . . . . . . . . . . . . 68

3.7 Uncertainty Analysis . . . . . . . . . . . . . . . . 69

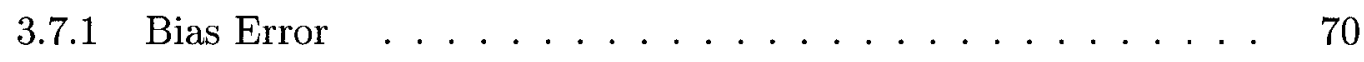

$3.7 .2 \quad$ Precision Error . . . . . . . . . . . . . . . 71

4 Results $\quad 73$

4.1 Gravimetric Analysis . . . . . . . . . . . . . . . 73

4.2 Spectral Line of Sight Attenuation . . . . . . . . . . 76 
CONTENTS

4.3 Determination of Scatter to Absorption Ratio . . . . . . . . . . . 80

4.3.1 Primary Particle Diameter from TEM . . . . . . . . . . 81

4.3.2 Soot Aggregate Geometric Parameters from SEM . . . . . . . 82

4.3.2.1 Fractal Parameters . . . . . . . . . . . 82

4.3.2.2 Aggregate Size Distribution .......... 83

4.3.3 Calculating $\rho_{s a}$ Results . . . . . . . . . . . . 84

4.4 Soot Absorption Function . . . . . . . . . . . . . . 90

4.5 LII Soot Volume Fractions . . . . . . . . . . . . . . . . . . 95

5 Discussion $\quad 98$

5.1 Effect of Distribution Choice . . . . . . . . . . . . . . 98

5.2 Comparison Among Conditions . . . . . . . . . . . . . . . 98

5.2.1 Variation with Wavelength . . . . . . . . . . . 100

5.3 Dimensionless Extinction . . . . . . . . . . . . . 102

5.4 Mass Absorption Cross-section . . . . . . . . . . . . . . . . . . 104

5.5 Comparison to Literature . . . . . . . . . . . . . . . 106

5.6 Implications . . . . . . . . . . . . . . . . . . . 109

6 Conclusions and Recommendations 113

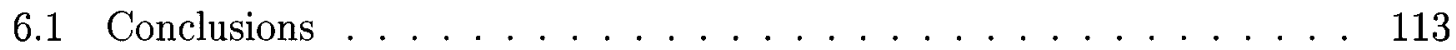

6.2 Recommendations . . . . . . . . . . . . . . . . . 115

$\begin{array}{ll}\text { References } & 117\end{array}$

$\begin{array}{ll}\text { A Burner Repeatability } & 127\end{array}$

B Mathcad Analysis Files $\quad 129$

B.1 Fitting Aggregate Size Distributions . . . . . . . . . . . . . . . 129 
B.2 Scatter-to-Absorption Ratio . . . . . . . . . . . 136

B.2.1 Reference Sheet . . . . . . . . . . . . . 136

B.2.2 Calculation of $\rho_{s a, \lambda} \ldots \ldots \ldots \ldots$

B.3 LOSA Analysis . . . . . . . . . . . . . . . . . . 145

C Correlations in the Results $\quad 161$

D Correlations in Optical and Fuel Properties $\quad 167$ 


\section{List of Tables}

1.1 Relevant studies of soot optical properties . . . . . . . . . . 18

3.1 Summary of Burner Conditions . . . . . . . . . . . . . . 44

4.1 Gravimetric soot volume fractions, in parts per billion (ppb) . . . . 73

4.2 Values of the mass density of soot from literature . . . . . . . . . 75

4.3 Primary particle diameters as measured by TEM . . . . . . . . . 81

4.4 Fractal properties as measured by SEM . . . . . . . . . . . 82

4.5 Soot aggregate size distribution parameters for log-normal $\left(N_{g}, \sigma_{2 g}\right)$ and self-preserving $\left(M_{1}, \tau\right)$ distributions . . . . . . . . . 83

4.6 The $95 \%$ confidence limits of $\rho_{s a}$ parameters for case C3 . . . . 90

5.1 Summary of condition-averaged results . . . . . . . . . . . 106

viii 


\section{List of Figures}

1.1 Effect of laser fluence on reported soot volume fraction for in-flame and ambient soot $[$ Smallwood, 2007] . . . . . . . . . . . . . 12

1.2 A comparison of $E(m)_{\lambda}$ values from literature. Samples for all ex-situ measurements were collected from within a flame. . . . . . . . .

2.1 Angular scattering $\left(I_{s c a}\right)$ diagrams for polystyrene spheres in water, with diameters of (a) $50 \mathrm{~nm}$, (b) $500 \mathrm{~nm}$, and (c) $2000 \mathrm{~nm}$. Laser light at $632.8 \mathrm{~nm}$ is incident on the left-hand side, and scatter angle is measured counter-clockwise from the forward direction [Morrison and Ross, 2002]. . . . . . . . . . . . . . . .

3.1 (a) Layout of the burner system and (b) cross-section of the burner head. Dimensions are in millimeters. . . . . . . . . . . . . . .

3.2 The combustion chamber of the inverted burner in (a) a solid model and (b) a photograph ..................... 41

3.3 Photograph of the burner setup . . . . . . . . . . . . . . . 42

3.4 (a) Solid model rendering and (b) schematic of the isokinetic sampling

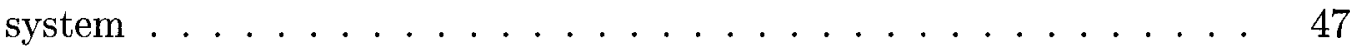

3.5 Typical TEM image used for $d_{p}$ measurement . . . . . . . . . . . 49

3.6 SEM image (a) before and (b) after application of the flatten filter . . 52 ix 
3.7 SEM image after application of the median filters . . . . . . . . . 52

3.8 Aggregate in original SEM micrograph image . . . . . . . . . . 53

3.9 Result of thresholding an unprocessed SEM micrograph . . . . . . . . 54

3.10 Aggregate in processed SEM micrograph . . . . . . . . . . . 54

3.11 Aggregate as measured overlaid on original . . . . . . . . . . . 55

3.12 Misrepresented aggregate in (a) original micrograph, and (b) overlaid

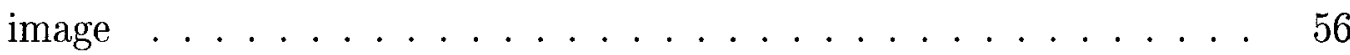

3.13 Experimental measurements and determinable parameters. Note that the overlap coefficient $C_{o v}$ and the surface area $S_{a}$ were not used in the present analysis. . . . . . . . . . . . . . . 58

3.14 Number of primary particles versus aggregate length (condition A1) . 58

3.15 Fits of (a) log-normal and (b) self-preserving distributions to the aggregate size data . . . . . . . . . . . . . . 60

3.16 Schematic of Spec-LOSA experiment . . . . . . . . . . . . . 64

3.17 Photograph of LOSA setup . . . . . . . . . . . . . . . . 64

3.18 Typical unfiltered (low-wavelength set) Spec-LOSA image . . . . . 65

3.19 Normalized transmissivity profile across plume (condition D3) . . . . 66

3.20 Smoothed transmissivity profile from centreline (condition D3) . . . 67

3.21 Radial profile found by tomographic inversion (condition D3). The vertical dashed line indicates the edge of the burner exit nozzle. . . . 67

3.22 Schematic of LII system [Snelling et al., 2005] . . . . . . . . . . 69

4.1 Specular extinction coefficients for condition A1 . . . . . . . . 77

4.2 $K_{\text {ext, } \lambda}$ data comparing dilution rates, with the C-series being the most diluted and $\mathrm{E}$ the least . . . . . . . . . . . . . . 78 
4.3 Comparison graph of all extinction data (refer to Table 3.1 on page 44 for further description of the burner conditions) $\ldots \ldots 79$

4.4 Normalized $\ln \left(\tau_{\lambda}\right)$ data for condition $C 2 \ldots \ldots$. . . . . . . . 80

4.5 Comparison of bias, precision, and combined uncertainties in $\ln \left(\tau_{\lambda}\right)$ measurements for condition $\mathrm{C}_{2} \ldots \ldots \ldots$. . . . . . . . . 81

4.6 Scatter-to-absorption ratio for condition $\mathrm{A} 1 \ldots \ldots . \ldots 8$

4.7 Scatter-to-absorption ratio data comparing dilution rates . . . . . . . 87

4.8 Scatter-to-absorption ratio results for all conditions, calculated using the log-normal distribution (refer to Table 3.1 on page 44 for further description of the burner conditions) $\ldots \ldots \ldots \ldots$

4.9 Scatter-to-absorption ratio results for all conditions, calculated using the self-preserving distribution (refer to Table 3.1 on page 44 for further description of the burner conditions) . . . . . . . . .

4.10 Soot absorption function results for condition A1 . . . . . . . . 91

4.11 Soot absorption function results, comparing dilution rates $\ldots$. . . . 92

4.12 Soot absorption function results for all conditions, with $\rho_{s a, \lambda}$ calculated using log-normal distribution (refer to Table 3.1 on page 44 for further description of the burner conditions) .............

4.13 Soot absorption function results for all conditions, with $\rho_{s a, \lambda}$ calculated using self-preserving distribution (refer to Table 3.1 on page 44 for further description of the burner conditions) . . . . . . . . . . . 94

4.14 LII soot volume fraction versus laser fluence, using flat $E(m)$ of 0.4 (refer to Table 3.1 on page 44 for further description of the burner

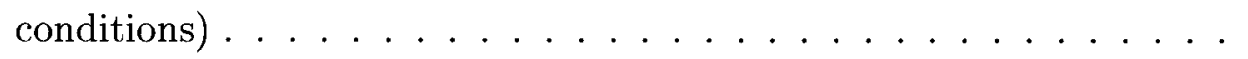


4.15 LII soot volume fraction versus laser fluence, using $E(m)_{\lambda}$ values from the present work (refer to Table 3.1 on page 44 for further description of the burner conditions) . . . . . . . . . . . . .

4.16 Comparison of peak soot volume fractions found by LII and gravimetric analysis

$5.1 E(m)$ results, treating the various conditions as repeated measurements 100

$5.2 \tilde{E}(m)$ results for all conditions, each wavelength range normalized by (a) the maximum value in that set and (b) the value corresponding to a wavelength of $700 \mathrm{~nm}$ in that set (refer to Table 3.1 on page 44 for further description of the burner conditions) . . . . . . . . . 103

5.3 Dimensionless extinction constant versus wavelength for all conditions (refer to Table 3.1 on page 44 for further description of the burner conditions) . . . . . . . . . . . . . . 105

5.4 Mass absorption cross-sections for all conditions (refer to Table 3.1 on page 44 for further description of the burner conditions) . . . . . 107

5.5 Condition-averaged $E(m)_{\lambda}$ data plotted alongside literature values. Samples for all ex-situ measurements were collected from within a flame.108

5.6 Comparison of present MAC results to the literature values reviewed and adjusted by Bond and Bergstrom [2006] . . . . . . . . . . 110

5.7 Comparison of peak soot volume fractions found by LII and gravimetric analysis ............................... 111

A.1 Repeated SMPS measurements of a singe condition . . . . . . . . . 128

C.1 Plot of $E(m)$ results versus gravimetric soot volume fraction, $f_{v} \ldots \ldots 162$

C.2 Plot of $E(m)$ results versus primary particle diameter, $d_{p} \ldots \ldots$. . . 162 
C.3 Plot of $E(m)$ results versus fractal dimension, $D_{f} \ldots \ldots \ldots \ldots$

C.4 Plot of $E(m)$ results versus fractal prefactor, $k_{g} \ldots \ldots \ldots \ldots$

C.5 Plot of $E(m)$ results versus radius of gyration, $R_{g} \ldots \ldots \ldots \ldots$

C.6 Plot of $E(m)$ results versus mean value of $N \ldots \ldots \ldots \ldots$

C.7 Plot of $E(m)$ results versus exhaust residence time $\ldots \ldots \ldots \ldots$

C.8 Plot of $E(m)$ results versus characteristic aggregation time, $t_{c} \ldots \ldots 165$

C.9 Plot of $E(m)$ results versus dilution ratio, $D R \ldots \ldots \ldots \ldots$

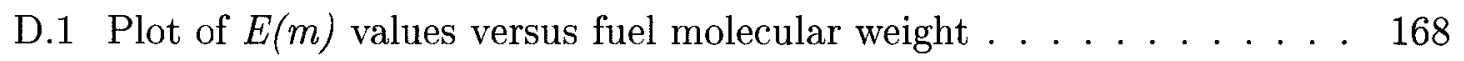

D.2 Plot of $E(m)$ values versus number of carbon atoms per molecule of fuel168

D.3 Plot of $E(m)$ values versus number of hydrogen atoms per molecule of fuel . . . . . . . . . . . . . . . . . . . . . . . . 169

D.4 Plot of $E(m)$ values versus a ratio of carbon to hydrogen $\ldots \ldots \ldots$ 


\title{
Nomenclature
}

\author{
Variables \\ $A_{a} \quad$ Aggregate projected area \\ $A_{p} \quad$ Primary particle projected area \\ $D_{f} \quad$ Fractal dimension \\ $E(m)$ Absorption refractive index function \\ $\tilde{E}(m)$ Relative $E(m)$ : spectral variation in $E(m)$ relative to a maximum value \\ $F(m)$ Scattering refractive index function \\ $\Gamma \quad$ The Gamma function, $\Gamma(z)=\int_{0}^{\infty} t^{z-1} e^{-t} d t$ \\ I Intensity of light \\ $K \quad$ Aggregation kernel \\ $K_{e} \quad$ Dimensionless extinction constant \\ $K_{a b s} \quad$ Volumetric absorption coefficient \\ $K_{\text {ext }} \quad$ Volumetric extinction coefficient \\ $K_{\text {sca }} \quad$ Volumetric scattering coefficient \\ $L \quad$ Optical path length through a medium \\ $M_{p} \quad$ Moment of a distribution; the subscript denotes the order of the moment \\ $N \quad$ Number of primary particles per aggregate \\ $\Omega \quad$ Solid angle \\ $P(N)$ Probability distribution function for $N$ \\ $R_{g} \quad$ Radius of gyration
}

xiv 
$S(q)$ The structure factor

$T$ Temperature

$U_{x} \quad$ Uncertainty limits of variable $x$

$V \quad$ Volume of exhaust gas

$c \quad$ The speed of light

$d_{p} \quad$ Primary particle diameter

$\mu \quad$ Dynamic viscosity

$f_{v} \quad$ Volume fraction (of soot)

$g(r)$ The density autocorrelation function

$h \quad$ The Planck constant

$k \quad$ The Boltzmann constant

$k_{f} \quad$ Fractal prefactor, defined using the primary particle radius

$k_{g} \quad$ Fractal prefactor, defined using the primary particle diameter

$l \quad$ Spacing between particles in a medium

$\lambda \quad$ Wavelength of light

$m \quad m=n+\imath k$, the complex index of refraction

$m_{s} \quad$ Mass of soot

$n \quad$ Aggregate number concentration per unit volume

$\phi \quad$ Azimuth angle, flame equivalence ratio

$q \quad$ The scattering wave vector

$\rho_{s} \quad$ Soot mass density

$\rho_{s a} \quad$ Ratio of scattering to absorption

$r_{i} \quad$ Position vector of particle $i$

$s \quad$ Position along the optical path

$s_{p} \quad$ Mean value of $N$, the subscript denoting what order of moment was used in its calculation 
$\sigma_{a b s} \quad$ Absorption cross-section

$\sigma_{e x t} \quad$ Extinction cross-section

$\sigma_{\text {sca }} \quad$ Scattering cross-section

$\tau \quad$ Optical transmissivity

$t_{c} \quad$ Characteristic time for coagulation

$\theta \quad$ Scattering angle

$x_{p} \quad$ Non-dimensional particle size parameter

Subscripts and Superscripts

abs Refers to absorption properties

agg Refers to aggregate properties

ext Refers to extinction properties

$\lambda \quad$ Denotes wavelength dependence

$p \quad$ Refers to individual primary particle properties

sca Refers to scattering properties

\section{Abbreviations}

CAC Criteria Air Contaminant

LII Laser-Induced Incandescence

MAC Mass absorption cross-section

MSC Mass scattering cross-section

PM Particulate matter

RDG-PFA Rayleigh-Debye-Gans theory for Polyfractal Aggregates

SFE Simple forcing efficiency

ppb Parts per billion

ppm Parts per million

DR Dilution ratio, $D R=Q_{d i l} / Q_{f u e l}+Q_{a i r}$ 


\section{Chapter 1}

\section{Introduction}

Global use of fossil fuels continues to increase, and there is an ever-growing need to regulate and mitigate the harmful emissions from their use. It is estimated that over $90 \%$ of the world's energy demands (roughly $460 \mathrm{EJ}$, or $4.6 \cdot 10^{20} \mathrm{~J}$, in 2004) are met by the combustion of various fuels [IEA, 2006], resulting in vast quantities of pollutants being released into the air. Many nations, including Canada, have passed legislation as a first step towards mitigation of the negative impact these substances have on the environment, such as the Canadian Environmental Protection Act, 1999 (CEPA 1999) [CEPA, 1999]. This act requires stationary sources to report the quantities of released Criteria Air Contaminants (CACs) to Environment Canada's National Pollutant Release Inventory (NPRI). These CACs include carbon monoxide (CO), oxides of nitrogen (NOx), oxides of sulphur (SOx), volatile organic compounds (VOCs), and particulate matter (PM).

Particulate matter has been shown to cause adverse health effects in humans and other animals [Pope III et al., 2002], and is therefore listed as a CAC. In general, to reflect the importance of size of PM in determining health impacts, emissions of PM are separated into four groups: total PM with diameter less than 100 microns, coarse 
PM with diameter less than or equal to 10 microns (PM10), fine PM with diameter less than or equal to 2.5 microns (PM2.5), and ultra-fine PM with diameter less than or equal to 0.1 microns (PM0.1). Carbonaceous soot from combustion processes generally falls into the latter two categories, PM2.5 and PM0.1 [Seinfeld and Pandis, 1998, Xi and Zhong, 2006].

A significant disconnect still exists between the existing requirement to report and any future efforts toward regulation and mitigation of PM emissions: in many cases, the emitted mass of this particulate aerosol has proven to be quite difficult to measure. An example of this is in the flaring of solution gas from oil fields, which is typically done through open-atmosphere non-premixed flames in a crosswind [Johnson et al., 2001]. The remote locations and inaccessible nature of flares have impeded efforts towards quantification of soot emissions; however, they are implicated as a significant source of PM due to their sheer magnitude. Flaring accounts for an estimated 150 billion cubic meters of solution gas burnt per year worldwide [Elvidge et al., 2007], with 0.723 billion cubic meters flared in Alberta alone in 2007 [ERCB, 2008]. While the composition of solution gas varies dramatically, it is predominantly methane. It is for this reason that methane is the chosen fuel for this research.

\subsection{Soot Morphology}

Soot particles consist of many small carbonaceous spherules of near-constant diameter, called primary particles, aggregated together in a branching, chain-like structure. The formation mechanisms of these primary particles are complex and the details are not well understood [e.g. Appel et al., 2000, Bockhorn et al., 2002]. Conceptually, the process consists of four steps: formation of aromatic hydrocarbons, soot particle inception, soot growth and coagulation, and soot oxidation [Bockhorn, 1994]. The 
formation process begins when hydrocarbon fuels degrade during oxidation to produce small hydrocarbon radicals. These radicals combine under fuel-rich conditions to produce small hydrocarbons (particularly acetylene), which, when sufficient carbon atoms are present, eventually combine to form aromatic rings. These rings continue to grow, primarily via the addition of acetylene molecules, resulting in coagulation into soot primary particles. Particle growth continues both by ongoing coagulation and through surface growth, whereby additional molecules are picked up from the gas phase. Particles are destroyed by oxidation in fuel-lean regions, particularly where temperatures exceed 1300 K[Bockhorn, 1994]. Typically, the bulk of the formed soot is consumed in this oxidation process - for example, only a few thousandths of the initial total end up being emitted from diesel engines [Bockhorn et al., 2002].

The physical structure and geometry of soot aggregates are such that they can not be approximated successfully using dense or porous spheres, or any other simple shapes [Filippov et al., 2000]. It has been shown [e.g. Megaridis and Dobbins, 1990, Köylü and Faeth, 1992] that soot aggregates are well-described as mass-fractal-like, where the number of primary particles, $N$, that make up an aggregate, can be related to the radius of gyration, $R_{g}$, and the primary particle diameter, $d_{p}$, by Equation (1.1), where $D_{f}$ is the fractal dimension and $k_{g}$ is the fractal prefactor ${ }^{1}$ :

$$
N=k_{g}\left(\frac{R_{g}}{d_{p}}\right)^{D_{f}}
$$

Typical values of the fractal parameters for soot are $7<k_{g}<9$ and $1.6<D_{f}<1.9$,

\footnotetext{
${ }^{1}$ Some researchers [e.g. Brasil et al., 1999, Filippov et al., 2000, Sorensen, 2001] adopt an alternate definition using the primary particle radius, $a$, in place of the diameter, which results in different values for the fractal prefactor. One might argue that this definition is more consistent in that it contains a ratio of radii; however, the diameter is the measured quantity and it is desirable to use that value directly. To avoid confusion, we will adopt the term $k_{g}$ as the prefactor defined using the diameter as in Equation (1.1) and $k_{f}$ as the prefactor when using the radius instead. The relationship between the two is $k_{f}=k_{g}(1 / 2)^{D_{f}}$.
} 
with average values generally taken to be $k_{g}=8$ and $D_{f}=1.8$ [Modest, 2003, and references therein]. Equation (1.1) is useful because it relates $N$ and $R_{g}$ using only experimentally-determinable constants. The radius of gyration, which represents the root-mean-squared distance of the primary particles from their aggregate centroid, is

$$
R_{g}^{2}=\frac{1}{N} \sum_{i=1}^{N} r_{i}^{2}
$$

where $r_{i}$ is the radial position of particle $i$ relative to the centroid. $R_{g}$ is an important parameter when considering light scattering, as will be seen in Section 2.4.1.

\subsubsection{Soot Particle Agglomeration}

The agglomeration of aerosols and colloids occurs due to collisions resulting from random (Brownian) motion. In cases where particles stick together with near-unity probability, the rate of diffusive motion of the particles is the limiting factor. This condition is referred to as diffusion-limited cluster aggregation (DCLA), and typically results in aggregates with fractal dimensions of $D_{f} \simeq 1.75-1.8$ [Sorensen, 2001, and references therein]. The agglomeration of soot primary particles into fractal-like clusters is a case of DCLA.

An important parameter in aggregation is the characteristic time for coagulation, denoted $t_{c}$, which is defined as the time taken for the number concentration of aggregates to halve. This parameter is given by [Seinfeld and Pandis, 1998]

$$
t_{c}=\frac{2}{K \cdot n_{0}}
$$

where $n_{0}$ is the initial aggregate number concentration and $K$ is the aggregation 
kernel, given by

$$
K=\frac{8 k T}{3 \mu(T)}
$$

where $k$ is the Boltzmann constant, $T$ is the gas temperature and $\mu$ the gas viscosity. It follows then that the number concentration of aggregates is a function of the initial concentration and the coagulation time. Additionally, the aggregation kernel is directly dependent on temperature and viscosity, which in turn is also a function of temperature. However, since the total number of primary particles remains effectively constant, it follows that aggregate size $N$ is a function of temperature, residence time, and concentration as well. One implication of this is that the soot measured in-flame and shortly post-flame, where temperatures are quite high, is still undergoing rapid aggregation. Thus, size measurements made at these locations may be significantly different than soot emitted from a tailpipe or smokestack, for example, where elevated temperatures and comparatively long residence times can lead to greater degrees of aggregation. Because of this, it is desirable to measure the optical properties of soot that has been allowed to cool in the exhaust stream, to better represent actual soot emissions from such sources.

\subsubsection{Aggregate Size Probability Distributions}

A probability distribution function (PDF), expressed as $P(N ; x, u x)$, whose arguments $x$ and $u x$ control the distribution shape, is used to describe the probability that a randomly selected aggregate contains $N$ primary particles. This distribution, when multiplied by the number concentration of aggregates, $n$, gives the size distribution of aggregates per unit volume. The product $n N$ is called the aggregate size distribution, which describes the number concentration of aggregates as a function of $N$. 
There is some contention over which probability distribution best describes soot populations. The most commonly used is the log-normal distribution, in which the logarithm is normally distributed, and which has the form [Köylü and Faeth, 1992],

$$
P_{L N}\left(N ; N_{g}, \sigma_{2 g}\right)=\frac{1}{N \cdot \ln \left(\sigma_{2 g}\right) \cdot \sqrt{2 \pi}} \exp \left\{-\left[\frac{\ln (N)-\ln \left(N_{g}\right)}{\sqrt{2} \ln \left(\sigma_{2 g}\right)}\right]^{2}\right\}
$$

where $x$ and $u x$ in this case are $N_{g}$ and $\sigma_{2 g}$, the geometric mean and standard deviation of the free variable $N$. It is well-established, however, that the self-preserving, scaling distribution provides a better description of aggregating systems [Friedlander and Wang, 1966, Sorensen, 2001]. As is often the case, the caveat here is that the self-preserving distribution is significantly more complex. The basic form of this distribution is

$$
\begin{aligned}
P_{S P} & =\frac{M_{1}}{s_{p}^{2}} \phi\left(\frac{N}{s_{p}}\right) \\
\text { where } \phi\left(\frac{N}{s_{p}}\right) & =A\left(\frac{N}{s_{p}}\right)^{-\tau} \exp \left(-\alpha \frac{N}{s_{p}}\right)
\end{aligned}
$$

$M_{1}$ is the first moment of the distribution, the general ( $p$-th order) form of which is

$$
M_{p}=\int N^{p} P(N ; x, u x) d N
$$

The factor $A=\alpha^{2-\tau} / \Gamma(2-\tau)$, where $\Gamma$ is the Gamma function, $\tau$ is a measure of the width of the distribution, $\alpha=p-\tau$, and $s_{p}=M_{p} / M_{p-1}$ is a mean value of $N$ based on the $p$-th moment of the distribution. It is worth noting that this form of the distribution is only valid for $N / s_{p}>1$. However, light scattering weights aggregate size heavily, so aggregates with small $N$ (meaning $N / s_{p}<1$ ) can be safely ignored [Sorensen, 2001]. If we define the mean aggregate size using the first moment, $p=1$, then $s_{1}=M_{1} / M_{0}$ 
or simply $s_{1}=M_{1}$ since $M_{0}=1$. Combining and expanding Equations (1.6) and (1.7) using $p=1$, the self-preserving distribution reduces to the following form with only two input variables:

$$
P_{S P}\left(N ; M_{1}, \tau\right)=\frac{1}{M_{1}}\left\{\frac{(1-\tau)^{2-\tau}}{\Gamma(2-\tau)}\left(\frac{N}{M_{1}}\right)^{-\tau} \exp \left[\frac{-N(1-\tau)}{M_{1}}\right]\right\}
$$

According to Sorensen [2001], the more intuitive log-normal distribution is a reasonable approximation for up to the second moment $(p=2)$, but is generally inaccurate for higher moments. A notable exception, where the log-normal distribution is reasonable at moments higher than the second, occurs when the width parameter is $\sigma_{2 g} \approx 2.4$. Since scattering does indeed consider higher moments and typical lognormal widths found by TEM are considerably higher [e.g. Köylü and Faeth, 1994a], the self-preserving distribution provides a more accurate description of the aggregate population. In an effort to make a comparison between the two, both distributions are used in the present analysis and the results compared in Chapter 4.

\subsection{Methods of Soot Emission Measurement}

The current standard for measuring soot emission from flares remains the United States Environmental Protection Agency (EPA) Test Method 9, a human observationbased opacity test [E.P.A., 1990]. This method is lacks both rigor and accuracy, and is one example of why development of accurate soot diagnostics is a subject of worldwide research.

Methods used in the measurement of soot emission can be divided into two broad categories: in-situ and ex-situ measurements. Ex-situ measurements are those that require extractive sampling and are thus limited in temporal resolution. Moreover, 
extractive methods are in general limited to point measurements, and require direct physical access to the soot source for collection. In contrast, in-situ measurements are performed directly on the flame or soot plume, and have several advantages over extractive methods. For example, they are comparatively fast, allowing for some measure of temporal resolution and feedback. Light-based in-situ methods require only optical access to the soot source, which makes remote sensing possible. Despite these advantages, the accuracy of optical methods hinge on accurate knowledge of soot optical properties, which has proven problematic and remains a significant barrier to widespread implementation.

The objective of this research is to measure the optical properties of cooled soot from a methane flame, as an approximation to the soot emitted from solution gas flares and other natural gas diffusion flames. Several relevant methods of measuring soot emissions and properties are outlined in the following sections. Ex-situ methods are discussed first, followed by some popular in-situ methods.

\subsubsection{Gravimetric Analysis}

Gravimetric analysis is an ex-situ technique in which all particles from a known gas volume are collected and weighed to determine the mass of soot collected within a certain time interval. This is typically achieved through filter sampling of the aerosol while monitoring the gas velocity and sampling duration. If the particle density is known, and the total gas volume drawn through the filter is measured, a non-dimensional volume fraction, $f_{v}$, can be obtained that is independent of the optical properties of the particles. For combustion-generated particulates, the volume fraction is generally on the order of parts per million to parts per billion. Methane, the fuel of choice for this work, has the lowest sooting propensity of hydrocarbon fuels, 
so the concentrations encountered in methane exhaust are typically on the lower end of the spectrum, measured in parts per billion.

The gravimetric soot volume fraction can be used to calibrate optical diagnostics by inverting the governing equation to solve for the missing property information rather than the soot volume fraction. This method has been used in this way in the past, notably by Choi et al. [1995] and Krishnan et al. [2000].

\subsubsection{Electron Microscopy}

The physical structure of soot can be found by extractive sampling and observation via electron microscopy. Studies have found that three-dimensional properties of aggregates can be inferred from two-dimensional projected images [e.g. Köylü et al., 1995a], and were reviewed by Brasil et al. [1999] who outline a "recipe" for aggregate characterization. Details of the Brasil recipe can be found in Section 3.3.4. Transmission electron microscopy (TEM) is the current standard method used to determine soot morphology [e.g. Köylü et al., 1995b,a]. Soot is usually collected on carboncoated copper TEM grids, which are oriented parallel to the flow and rapidly inserted into the soot stream. The sampling duration is found by trial-and-error to yield an appropriate level of surface coverage, generally considered to be less than $10 \%$ [e.g. Wu et al., 1997]. Higher coverage levels mean that aggregates are more likely to overlap and be counted as one, whereas too little coverage has the disadvantage of an increased number of images required to obtain a representative sample.

The key mechanism for this method of soot collection is thermophoresis (also known as thermodiffusion or the Soret effect), which is the mechanism whereby particles migrate from a hot region to a cold one [Duhr and Braun, 2006]. It has been previously shown [Dobbins and Megaridis, 1987] that thermophoretic sampling col- 
lects a representative sample, meaning that it is not biased with respect to particle size, making it the method of choice for sampling soot from hot gases. However, in the current case of a cooled exhaust stream, the thermal gradient between the exhaust gases and the TEM grid at ambient temperature is small, resulting in a weak thermophoretic force. This called into question the unbiased nature of this method, and ultimately led to the conclusion that thermophoretic sampling is not appropriate for finding the aggregate size distribution in this case. Instead, filter sampling and subsequent analysis by scanning electron microscope (SEM) was performed.

However, TEM was used to determine the mean primary particle diameter, which is assumed to be relatively constant for all aggregates in a sample. For this purpose, the possibility of biased sampling for TEM is irrelevant since the primary particle diameter is independent of aggregate size and can be measured from any sample of aggregates.

\subsubsection{Laser-Induced Incandescence}

The first in-situ measurement discussed here is laser-induced incandescence (LII), an optical diagnostic that has gained worldwide popularity. LII works on the principle of measuring the incandescence of soot that has been rapidly heated by a laser pulse. By taking the ratio of intensities at two or more distinct wavelengths, the mean temperature of the particles can be found by pyrometry, and the temporal decay of the particle temperature can be used to infer particle size. Snelling et al. [2005] describe the theory behind a self-calibrating LII system, to which the reader is referred for further information; the theory and full analysis of LII is complex, and is given only a brief treatment here.

The soot volume fraction, $f_{v}$, found by LII is given by Equation (1.10) [Snelling 
et al., 2005]:

$$
f_{v}=\frac{V_{e x p}}{\eta w_{b} G_{E X P} \frac{12 \pi c^{2} h}{\lambda^{6}} E(m)_{\lambda}\left[\exp \left(\frac{h c}{k \lambda T_{p}}\right)-1\right]^{-1}}
$$

where $c$ is the speed of light, $h$ is the Planck constant, $k$ is the Boltzmann constant, $V_{E X P}$ is the experimental voltage, $\eta$ is a calibration factor, $w_{b}$ is the laser sheet thickness, $G_{E X P}$ is the experimental detector gain, and $T_{p}$ is the particle temperature, to be determined experimentally by taking a ratio of the incandescence intensities, discussed below. The term $E(m)_{\lambda}$ is called the absorption refractive index function, where $m=n-\imath k$ is the complex index of refraction of soot, and the subscript $\lambda$ indicates the wavelength at which to evaluate the function. $E(m)$ is a key property for absorption-based soot optical diagnostics such as LII, and is defined, along with its counterpart, the scattering refractive index function $F(m)_{\lambda}$, as follows:

$$
\begin{aligned}
& E(m)_{\lambda}=\Im\left(\frac{m_{\lambda}^{2}-1}{m_{\lambda}^{2}+2}\right) \\
& F(m)_{\lambda}=\left|\frac{m_{\lambda}^{2}-1}{m_{\lambda}^{2}+2}\right|^{2}
\end{aligned}
$$

The particle temperature is determined by taking a ratio of the incandescence intensities at two wavelengths, resulting in the following expression where only $T_{p}$ is unknown:

$$
\frac{\lambda_{2}^{6} E(m)_{\lambda_{2}}}{\lambda_{1}^{6} E(m)_{\lambda_{1}}} \exp \left[-\frac{h c}{k T_{p}}\left(\frac{1}{\lambda_{1}}-\frac{1}{\lambda_{2}}\right)\right]=\frac{V_{E X P, 1}}{V_{E X P, 2}} \frac{\eta_{2}}{\eta_{1}} \frac{G_{E X P, 2}}{G_{E X P, 1}}
$$

It can be seen from Equation (1.10) that the reported soot volume fraction found by LII is inversely proportional to the value of $E(m)$, and by Equation (1.13) that the particle temperature relies on knowledge of the spectral variation of $E(m)$, sometimes referred to as the relative $E(m)$. The uncertain knowledge of both the absolute and relative values of $E(m)$ is the dominant source of uncertainty for an LII diagnostic. 


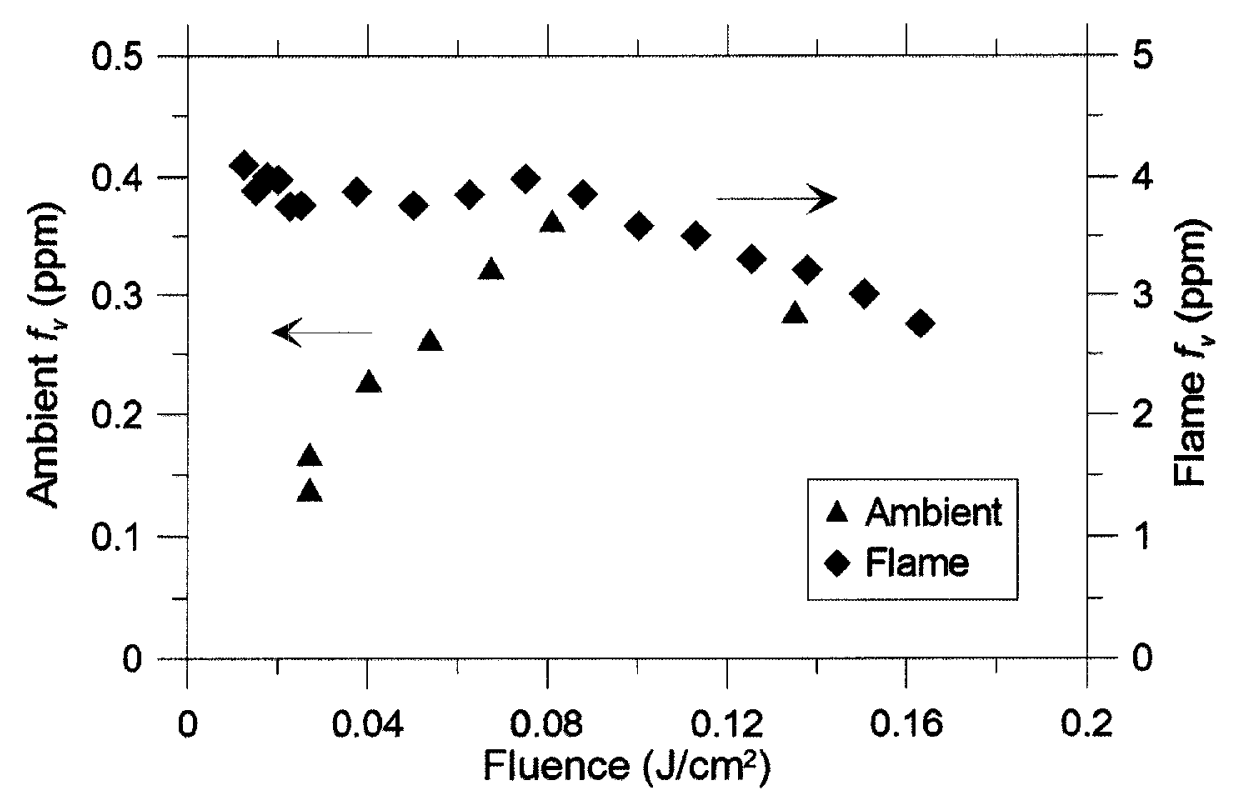

Figure 1.1: Effect of laser fluence on reported soot volume fraction for in-flame and ambient soot [Smallwood, 2007]

\subsubsection{Effects of Laser Fluence}

One particularly attractive feature of two-colour LII is the relative insensitivity of the diagnostic to the laser fluence, or energy per unit area. That is, the reported soot volume fraction is reasonably constant over a significant range of laser fluences - for in-flame soot [Vander Wal and Jensen, 1998]. For soot of ambient temperature, this is no longer the case; instead, the reported $f_{v}$ increases with increasing laser fluence, until sublimation temperatures are reached and the reported soot volume fraction drops off again; this trend is illustrated in Figure 1.1. It is therefore postulated that there is some temperature dependence on soot optical properties when the particles are allowed to cool in the exhaust stream. 


\subsubsection{Line of Sight Attenuation (LOSA)}

Line-of-sight attenuation is a robust optical extinction method that is used to determine the volumetric extinction coefficient via a transmissivity measurement. As discussed further in Chapter 2, the volumetric extinction coefficient can be related to the soot volume fraction by Equation (2.29), where the resulting soot volume fraction is again proportional to the value of $E(m)$. Transmissivity, $\tau$, is defined as the ratio of the intensities of transmitted light $\left(I_{\lambda}\right)$ to incident light $\left(I_{\lambda 0}\right)$, and relates to the extinction coefficient $K_{\text {ext }}$ using the Beer-Lambert Law [Siegell and Howell, 1981, Thomson et al., 2008b]:

$$
\tau_{\lambda}=\frac{I_{\lambda}}{I_{\lambda 0}}=\exp \left(-\int_{-\infty}^{\infty} K_{e x t, \lambda}(s) d s\right)
$$

where $s$ is the position along the optical path length through the medium, $L$. This means that the total integrated extinction coefficient along the optical path through the medium is simply a measurement of how much of the incident light did not make it to the detector. The validity of Equation 1.14 is constrained to low attenuations $\left(\int_{-\infty}^{\infty} K_{e x t, \lambda}(s) d s\right) \ll 1$, though this constraint can be softened when the scattering contribution is small) [Bohren and Huffman, 1983]. It will be shown in Section 3.5 that this condition is easily met in the present research. Spatially-resolved measurements can be made in one or two dimensions, using, for example, a spectrometer [Thomson et al., 2008a] or charge-coupled device (CCD) [Snelling et al., 1999], respectively. For the general case of the local extinction coefficient varying along the path length $s$, as it would for in-flame measurements, it may be possible, given certain assumptions, to invert the data using tomographic reconstruction to obtain a cross-sectional representation of the medium [e.g. Snelling et al., 1999, Ayranci et al., 2007, Thomson et al., 2008b]. However if the medium is uniform, the extinction coefficient loses its spatial 
dependence and comes out of the integral. For such a uniform medium, Equation (1.14) can be rearranged to solve for $K_{\text {ext, } \lambda}$ to obtain

$$
K_{e x t, \lambda}=\frac{-\ln \left(\tau_{\lambda}\right)}{L}
$$

\subsection{Current Knowledge of Soot Optical Properties}

The optical properties of soot have seen much attention in the past, and the methods used to obtain them are as widely varied as the results. It is often the case that values reported in literature are used generically, despite the specific nature of the experiments used to obtain these data. Table 1.1 summarizes several relevant previous studies discussed below, and Figure 1.2 compares the various values of $E(m)_{\lambda}$ found therein.

For the complex index of refraction of soot, $m=n-\imath k$, the commonly-used value of $m=1.57-0.56 \imath$ is often (mis)quoted as that reported in the early work of Dalzell and Sarofim [1969], who performed a polarization reflectance measurement on compacted soot pellets, referred to as "bulk" samples, which were extracted from propane and acetylene flames. In fact, Dalzell and Sarofim found spectrally-resolved values of $m$ for both acetylene and propane over the wavelength range of $435.8 \mathrm{~nm}$ to $10 \mu \mathrm{m}$, and found little variation in the visible wavelengths. According to Smyth and Shaddix [1996], this value was instead chosen by D'Alessio et al. [1973] as one that is "close to the value determined for soot produced by propane-air flames" found by Dalzell et al. [1970]. The spectrally-averaged values reported by Dalzell and Sarofim were $\tilde{m}=1.56-0.46 \imath$ for acetylene and $\tilde{m}=1.57-0.50 \imath$ for propane. Regardless, despite the popularity of these values, the validity of using compacted soot pellets has been questioned: it has been pointed out that the physical structure of the soot is altered 
in the compaction process, and that the void fraction must still be relatively high despite the intense pressure of compaction because it is a powder being compressed. This means that the index of refraction inferred from these measurements was that of the two-phase dispersion of soot and air rather than of the soot itself, and also that the sample surface can not be considered optically smooth at shorter wavelengths, so the use of the Fresnel equations becomes invalid for these conditions [Modest, 2003, p. 400 , and references therein].

Stagg and Charalampopoulos [1993] also used bulk soot samples from a premixed propane-oxygen flame to find the refractive index of soot, pyrolytic graphite, and amorphous carbon, using ellipsometric intensity measurements (a form of reflectivity measurement that detects the change in polarization of reflected light to infer the refractive index of the sample) over a range of temperatures $\left(25-600^{\circ} \mathrm{C}\right)$ and visible wavelengths $(400-700 \mathrm{~nm})$. They found little variation with either temperature or wavelength for these samples. Bulk samples were also used in reflectance measurements by Mullins and Williams [1987], who studied the variation in optical properties of soot from various fuels using both extinction and reflectance measurements. The extinction measurements were done on soot dispersed in glycerol in an ultrasonic bath, which breaks the aggregates into individual primary particles and distributes them evenly though the bath. They found that the extinction method provided more consistent results than the reflectivity method, due to uncertainties in the degree of compaction and surface roughness of the compacted samples. Little difference was found among soot from various fuels.

A dispersion model (dispersion theory predicts the wavelength dependence of $m$ based on the densities of bound and free electrons [Modest, 2003]) was developed by Lee and Tien [1981] and validated using polystyrene and Plexiglas soot data from previous work and from the literature. They found little variation in $m$ across the visible 
wavelengths, and found that at high temperatures there is little effect of temperature on optical properties, but lower temperatures exhibited different behaviour. Also noteworthy is that they found little difference between fuels with various hydrogento-carbon ratios, indicating that soots from different fuels are comparable.

Chang and Charalampopoulos [1990] performed combined scatter/extinction measurements at a single wavelength of $488 \mathrm{~nm}$, along with spectral extinction measurements over a range of wavelengths $(200-6400 \mathrm{~nm})$. The aggregate size distribution (assumed to be log-normal; see Section 1.1.2) was found via photon correlation measurements, allowing integration over the polydisperse population of aggregates as discussed in Section 2.4.3. They employed the Kramers-Krönig relationship [e.g. Roesslor, 1965] to solve for the complex refractive index, which required extrapolation of the experimental data to all wavelengths, introducing associated uncertainties.

Schnaiter et al. [2003] reported $m$ values for diesel soot, found by combined extinction and total scattering measurements at several wavelengths. Aggregate size distribution data were acquired by scanning mobility particle sizing (SMPS), which measures the distribution in terms of electrical mobility equivalent diameter. The conversion from electrical mobility diameter to a physical size parameter such as $R_{g}$ is non-trivial, however, and the use of mobility diameter at face value is in error. Bruce et al. [1991] also studied soot from open combustion of diesel fuel, at a very wide range of wavelengths, from $448 \mathrm{~nm}$ to $8.57 \mathrm{~mm}$, using a range of photoacoustic measurement to determine mass-normalized absorption and extinction coefficients.

In their work on the subject, Köylü and Faeth [1996] performed extinction measurements on soot in the over-fire region of buoyant turbulent diffusion flames burning various gaseous fuels. They employed the Rayleigh-Debye-Gans - Polydisperse Fractal Aggregate (RDG-PFA) approximation (see Section 2.4) to predict extinction coefficients, finding that the values of optical properties in the literature provide a poor 
fit to the experimental data. The best agreement was found with the work of Dalzell and Sarofim [1969].

Dobbins et al. [1994] performed specific extinction measurements on soot from a crude oil pool fire, at wavelengths of 450,630 , and $1000 \mathrm{~nm}$. They also performed an aging experiment, where a portion of the exhaust was drawn off into a chamber where it aged, and measurements were taken at various time intervals. They found no significant change in specific extinction coefficients over time.

Krishnan et al. [2000] studied soot from large buoyant turbulent diffusion flames, burning several gaseous and liquid fuels, using scattering and extinction measurements to determine the optical properties in the wavelength range of $400-800 \mathrm{~nm}$. Of particular interest is the provision of a correlation for the ratio of scattering to absorption refractive index functions $(F(m) / E(m)$, see Section 2.4). They also concluded that there is little variation in optical properties across fuel types and across visible wavelengths.

Snelling et al. [2004] applied optical pyrometry with low-fluence LII to soot within a laminar non-premixed ethylene flame in order to determine soot temperature and cooling rate, using an improved LII heat transfer model to infer values of $E(m)$ and the thermal accommodation coefficient $\alpha$. Soot morphology and size distribution were measured by thermophoretic sampling and transmission electron microscopy. Their models were unable to reproduce the experimental results with $E(m)$ from literature. Using the assumption of constant $E(m)$ with wavelength and that of linear variation with wavelength, they obtained results in the $E(m) \approx 0.4$ range.

To date, most work on soot optical properties has focused on soot sampled or measured in-flame rather than from an exhaust pipe or smoke stack, and, as discussed above, this does not necessarily reflect the latter conditions (nor ambient or atmospheric measurements, for that matter). In fact, Lee and Tien [1981] have shown 
Table 1.1: Relevant studies of soot optical properties

\begin{tabular}{|c|c|c|c|c|}
\hline Study & Fuel & $\lambda[\mathrm{nm}]$ & In/ex-situ & Method \\
\hline \hline Dalzell and Sarofim [1969] & $C_{2} H_{2}, C_{3} H_{6}$ & $436-10000$ & Ex-situ & Reflectance \\
\hline Lee and Tien [1981] & Polystyrene, Plexiglas & Visible & In-situ & Dispersion Model \\
\hline Mullins and Williams [1987] & Various & 450,633 & Ex-situ & Reflectance \\
\hline Chang and Charalampopoulos [1990] & $C_{3} H_{6} / O_{2}$ & $200-6400$ & In-situ & Scatter/extinction \\
\hline Bruce et al. [1991] & Diesel & Various & In-situ & Photoacoustic \\
\hline Stagg and Charalampopoulos [1993] & $C_{3} H_{6} / O_{2}$ & $400-700$ & Ex-situ & Elipsometry \\
\hline Dobbins et al. [1994] & Crude oil & $450-1000$ & In-situ & Extinction \\
\hline Köylü and Faeth [1996] & Various & $200-5200$ & In-situ & Extinction \\
\hline Krishnan et al. [2000] & Various & $351-800$ & In-situ & Scatter/extinction \\
\hline Schnaiter et al. [2003] & Diesel & $450-700$ & In-situ & Scatter/extinction \\
\hline Snelling et al. [2004] & Ethylene & $436-1064$ & In-situ & Pyrometry/LII \\
\hline
\end{tabular}

that, at least theoretically, there could be a significant change in optical properties between flame temperatures and ambient. This is evident in the use of LII, in that the reported soot volume fraction, $f_{v}$, is a strong function of laser energy for cooled soot - but not for in-flame soot. The purpose of this work is to extend the body of knowledge on soot optical properties to include that of cooled, post-flame soot from a methane flame, to better reflect the conditions seen in many applications including solution gas flaring.

\subsection{Objectives}

The accuracy of optical diagnostics is limited by the accurate knowledge of soot optical properties, in particular the absolute value and the spectral variation in the soot absorption refractive index function, $E(m)_{\lambda}$. It is hypothesized that this property varies with temperature or cooling history, suggested by the lack of agreement in experimental results between in-flame (hot) and near-ambient (cool) soot. Since it is often desirable to apply diagnostics to tailpipe emissions and atmospheric particu- 


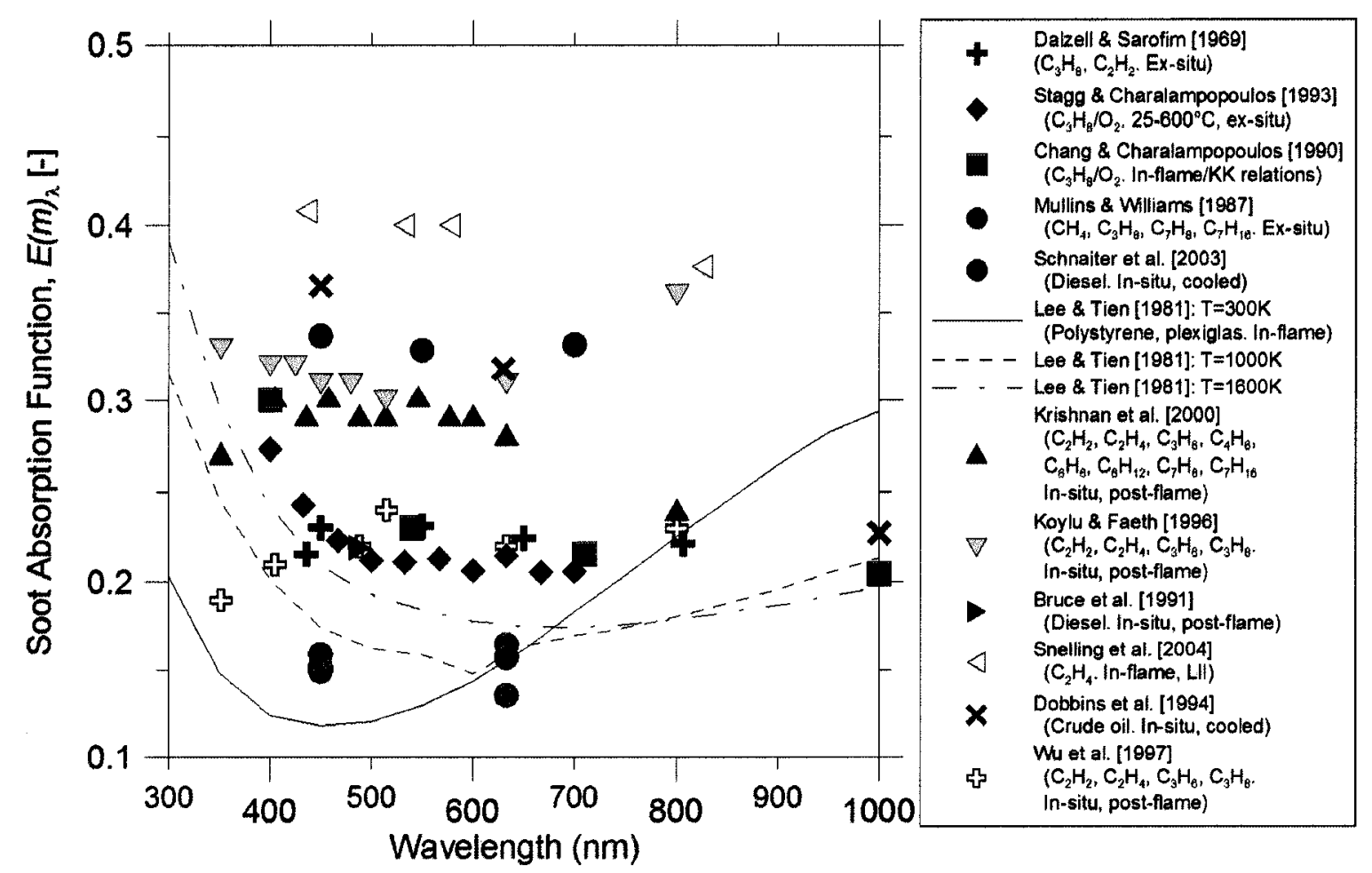

Figure 1.2: A comparison of $E(m)_{\lambda}$ values from literature. Samples for all ex-situ measurements were collected from within a flame. 
lates, measurements of $E(m)$ at ambient conditions are necessary. Soot from methane flames is rarely if ever considered, perhaps due to its low sooting propensity; however, the ubiquity of natural gas combustion warrants a more careful investigation of soot from this fuel. The effects of post-flame cooling history on optical properties are unknown, but experimental evidence (see Figure 1.1) shows an apparent distinction between flame-temperature and room-temperature soot. In fact, a significant change in optical properties with temperature was predicted by the theory of Lee and Tien [1981], yet not confirmed in experimental measurements by Stagg and Charalampopoulos [1993]; however, in the latter study, the bulk soot samples were collected shortly post-flame and later re-heated to make optical measurements at various temperatures. It is therefore postulated that in-stream cooling, and perhaps cooling rate, have an effect on soot optical properties. The goals of this research are therefore to measure the spectrally-resolved absorption function of soot originating from a methane diffusion flame that has been allowed to age and cool in the exhaust stream. To this end, the following goals are presented:

- Design and build the experimental apparatus, including an inverted co-flow diffusion flame soot generator for repeatable generation of particulate matter, and an isokinetic sampling system for filter-based soot extraction.

- Characterize the soot produced by the burner at a range of conditions with varying parameters by performing a range of diagnostics such that values of $E(m)_{\lambda}$ can be determined. From these measurements, attempt to identify effects of the varied parameters on the resulting optical property measurements.

- Conduct a detailed uncertainty analysis to estimate the accuracy of the results.

- Compare results to values in literature in order to identify any apparent trends. 
Chapter 2 outlines the relevant optical theory for the interaction of light with fractal aggregates like soot. Details of the experimental approach, implementation, and data reduction are presented in Chapter 3. Results are presented in Chapter 4, followed by discussion in Chapter 5, and conclusions and recommendations in Chapter 6 . 


\section{Chapter 2}

\section{Light-Particle Interactions}

An understanding of how light interacts with particles is fundamental to the application of optical diagnostics for particulate measurement. This chapter is intended to give an overview of the relevant theory, building up from interactions with individual atoms to the approximate relations used for a population of polydisperse, mass-fractal-like aggregates. The most widely accepted such approximation is the socalled Rayleigh-Debye-Gans approximation for fractal aggregates [Köylü and Faeth, 1992, 1994a, Sorensen, 2001]. This approximation is discussed below, and is the one ultimately used in the data analysis.

\subsection{Scattering and Absorption of Light by Particles}

On an atomic level, light incident on an atom can produce two different phenomena, depending on the wavelength (or photon energy) of the light. The general case is that the light is scattered, meaning that the light may be redirected but is left otherwise unchanged (called elastic scattering). Three mechanisms can contribute to the total directional change: diffraction can change the path of the photon without it actually 
colliding with the particle; reflection changes the direction through a particle surface interaction; and refraction can change the direction of a photon that penetrates the particle [Modest, 2003]. The vast majority of scattering is done elastically, meaning that there is no change in the wavelength or energy of the scattered light, though some depolarization can occur [Young, 1982]. For most engineering applications, nonelastic scattering is negligible and hereafter the term scattering will refer to elastic scattering.

A second type of interaction occurs if the energy of the photon matches that required to move the atom to a higher energy state. In this case, the photon is absorbed. Put another way, absorption occurs when the frequency of the incident light matches a resonant frequency of the atom, causing that atom to absorb the energy of the photon by jumping to an excited state. Every atom has at least one resonant frequency at which it will absorb radiation in this manner [Hecht, 2002]. In this regard, absorption is relatively simple - one simply has to know the wavelength of light and the absorptive properties of the medium.

To quantify the magnitude and direction of scattered light one must consider four parameters [Modest, 2003]:

1. The shape of the particle (generally assumed to be spherical for most particles and cylindrical for long, fibrous particles),

2. The material properties of the particle,

3. The relative size of the particle, and

4. The clearance between particles (ie., the concentration).

In the case of soot, aggregates are composed of primary particles which are assumed to be spherical, and the material properties are encompassed in the complex index of 
refraction, $m$. The relative size of a particle can be expressed as a non-dimensional size parameter, $x_{p}$, by the following expression, where $\lambda$ is the wavelength of light:

$$
x_{p}=\frac{\pi d_{p}}{\lambda}
$$

The spacing between particles can be expressed as a non-dimensional ratio $l / \lambda$, where $l$ is the clearance between particles in the system. In cases where $l_{\lambda}>0.5$ or $f_{v}<0.006$ (6000 parts per million, or ppm), this last parameter is not needed [Modest, 2003].

The amount of light absorbed or scattered by a particle can be described quantitatively by so-called cross-sections, which describe the effective area of the particle as seen by the incident light. The absorption cross-section, $\sigma_{a b s}$, and its counterpart, the scattering cross-section, $\sigma_{s c a}$, combine to describe the total extinction cross-section, $\sigma_{e x t}$, which is simply a sum of the absorptive and scattering contributions:

$$
\sigma_{e x t}=\sigma_{a b s}+\sigma_{s c a}
$$

\subsection{Rayleigh Theory}

In the latter half of the 19th century, British physicist Lord Rayleigh studied the interaction of light with air molecules (which are effectively tiny particles), and found that for very small particles, scattering is uniform in all directions and the scattered intensity is proportional to the inverse fourth power of wavelength $\left(I_{s c a} \propto 1 / \lambda^{4}\right)$. Rayleigh theory also says that absorption is volumetric, which allows the use of absorption measurements to determine the particle volume, from which the concentration and mass can be calculated. This simple theory is valid within the so-called Rayleigh limit $\left(x_{p} \ll 1\right)$, generally taken to be $x_{p}<0.3$ [Siegell and Howell, 1981]. Soot primary 
particles fall within this limit for all but the largest of particles at the smallest of wavelengths, and can be taken to hold, at least approximately, for the general case of soot at visible wavelengths [Modest, 2003]. However, problems arise when soot aggregates contain a large number of primary particles, such that the overall diameter of the aggregate falls outside the Rayleigh limit. For this reason, other scattering theories must be considered.

\subsection{Mie Theory}

The scattering from a single sphere with a diameter on the same order as the wavelength of the incident light is described by Mie theory, named after the work of Gustav Mie in the early 20th century. Mie theory is considerably more complicated than Rayleigh scattering. It is suggested that Mie theory is valid for the size range of $0.3<x_{p}<5$ [Siegell and Howell, 1981]. Larger particles $\left(x_{p} \gg 1\right)$ can be treated as a normal surface, where properties can be found using geometric reflection relations, whereas the small-particle limit of Mie theory approaches the Rayleigh scattering regime when $x_{p} \ll 1$. [Modest, 2003].

Mie scattering varies less with wavelength than Rayleigh scattering, but introduces strong angular dependence on $I_{s c a}$. Scattering is always strongest in the forward direction, which is particularly emphasized for larger particles. This can be seen in Figure 2.1, which compares the magnitude and direction of scattered light from polystyrene spheres of three different diameters, suspended in water with incident laser light coming from the left.

Further complications arise when soot aggregates are considered as a whole, due to particle-particle interaction (multiple scattering) and the complex aggregate structure. Attempts to apply Mie theory to a single sphere of equivalent volume to that 


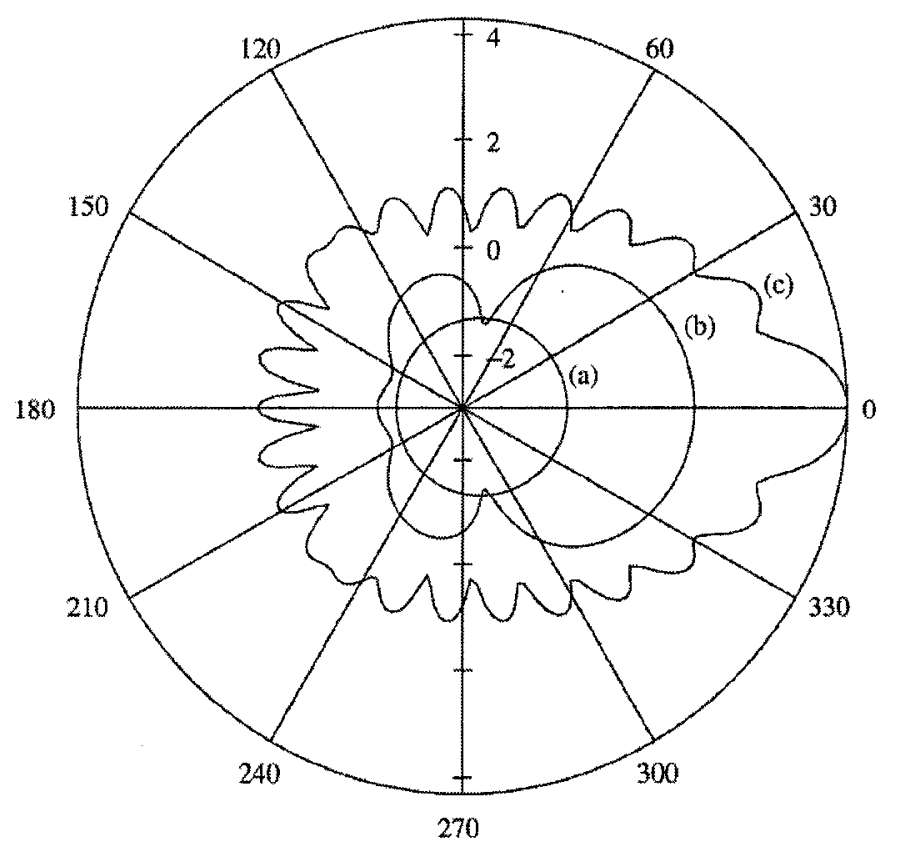

Figure 2.1: Angular scattering $\left(I_{s c a}\right)$ diagrams for polystyrene spheres in water, with diameters of (a) $50 \mathrm{~nm}$, (b) $500 \mathrm{~nm}$, and (c) $2000 \mathrm{~nm}$. Laser light at $632.8 \mathrm{~nm}$ is incident on the left-hand side, and scatter angle is measured counter-clockwise from the forward direction [Morrison and Ross, 2002]. 
of an aggregate (called the volume-equivalent sphere method) have resulted in erroneous predictions [Modest, 2003]. The convenient volumetric absorption seen in the Rayleigh regime also becomes invalid in the Mie regime. This results in the Mie theory being inappropriate for describing light-soot interaction, leading researchers to develop approximate relations that better describe how soot aggregates interact with light. Of them, the more rigorous solutions require detailed knowledge of the aggregate structure in order to consider multiple scattering (i.e., light scattered from one particle interacting with another). Such approximations include the volume integral equation formulation for scattering (VIEF) [Iskander et al., 1989] and the discretedipole approximation (DDA) [Draine and Flateau, 1994]. These approximations can be applied to simulated aggregates in numerical models, where the exact structure of

the aggregates are known. However, such a-priori knowledge of aggregate structure is impossible for the experimentalist to obtain, resulting in the need for a simpler approximation for use in the laboratory. The most widely-used such approximation is the Rayleigh-Debye-Gans theory for polydisperse fractal aggregates (RDG-PFA), which is the subject of the following section.

\section{$2.4 \quad$ RDG-PFA Approximation}

When it was found that neither Rayleigh nor Mie theory gave accurate predictions of scattering from soot, an approximate Rayleigh-Debye-Gans scattering model for polydisperse fractal aggregates (RDG-PFA) was developed [Bohren and Huffman, 1983, Dobbins and Megaridis, 1991, Köylü and Faeth, 1994a]. RDG-PFA theory provides a relatively simple method of approximating scattering from polydisperse fractal aggregates. This theory is based on the assumptions that each primary particle acts independently (i.e., there is no multiple scattering), that each particle is equally 
exposed to the incident light (i.e., there is no shielding of particles by other particles), and that the primary particles fall within the Rayleigh limit $\left(x_{p} \ll 1\right.$, taken as $\left.x_{p}<0.3\right)$ [Yon et al., 2008]. What follows is a summary of the theory, based heavily on the highly-regarded review written by Sorensen [2001], to which the reader is referred for a full derivation.

The absorption contribution of a particular aggregate is simply the contribution of a single primary particle (it is implicitly assumed that the primary particles in a given aggregate all have the same diameter) multiplied by the number of particles that make up the aggregate, $N$. If $\sigma_{a b s, \lambda}^{p}$ is the absorption cross section of a particle, then the absorption cross-section of an aggregate, $\sigma_{a b s, \lambda}^{a g g}$ is:

$$
\begin{aligned}
\sigma_{a b s, \lambda}^{a g g} & =N \sigma_{a b s, \lambda}^{p} \\
\text { where } \sigma_{a b s, \lambda}^{p} & =\frac{\pi^{2} d_{p}^{3}}{\lambda} E(m)_{\lambda}
\end{aligned}
$$

The subscript $\lambda$ is introduced here to emphasize the wavelength dependence of these variables, which is explored in the present research, and to distinguish these from their equivalent bulk properties (averaged across all wavelengths). Further, it can be noted from Equation (2.4) that absorption is volumetric and depends only on the number of particles, irrespective of aggregate structure so long as it remains within the Rayleigh limit.

Scattering is a more complex phenomenon, and the scattering cross-section of a particle, $\sigma_{s c a, \lambda}^{p}$ is a function not only of the particle size and the wavelength of light, but scattering direction and aggregate structure as well. The aggregate differential scattering cross-section, $d \sigma_{s c a, \lambda}^{a g g}$, by differential solid angle, $d \Omega$ is given by the following:

$$
\frac{d \sigma_{s c a, \lambda}^{a g g}}{d \Omega}=N^{2} \frac{d \sigma_{s c a, \lambda}^{p}}{d \Omega} S(q)
$$




$$
\text { where } \begin{aligned}
\frac{d \sigma_{s c a, \lambda}^{p}}{d \Omega} & =\frac{\pi^{4} d_{p}^{6}}{4 \lambda^{4}} F(m)_{\lambda} \\
d \Omega & =d(\cos \phi) d \theta
\end{aligned}
$$

and $\phi$ is the azimuth angle. The function $S(q)$ is called the structure factor, discussed in the following section. For non-polarized light, the total scattering from an aggregate can then be found by integrating Equation (2.5) over the range of solid angles ( $4 \pi$ steradians);

$$
\sigma_{s c a, \lambda}^{a g g}=\int \frac{d \sigma_{s c a, \lambda}^{a g g}}{d \Omega} d \Omega=\int_{4 \pi} N^{2} \frac{d \sigma_{s c a, \lambda}^{p}}{d \Omega} S(q) d \Omega
$$

This integration of the structure factor looks innocuous enough in Equation (2.8), but it is in fact a tricky one due to the structure factor's dependence on both scattering angle $\theta$ (through the wave vector $q$, discussed in the following section) and the aggregate size as expressed by $R_{g}$. Several authors have reported integrated forms of Equation (2.8) [eg, Dobbins and Megaridis, 1991, Köylï and Faeth, 1994a], which have been successfully applied to specific data but lack generalization, and many of them having singularities at $D_{f}=2$ [Sorensen, 2001]; thus, the integrated forms are not discussed here.

\subsubsection{Scattering Regimes and the Structure Factor}

The structure factor represents information about the size and structure of the scatterer or system of scatterers, as it relates to the wavelength of light being scattered [Sorensen, 2001]. The structure factor can take on several forms, depending on which of the so-called scattering regimes best describes the scenario. As mentioned above, scattering from a sphere is a function of particle size, scattering direction, and wavelength of light. The latter two are related by the scattering wave vector, $\vec{q}$, which 
represents the vector difference between the wave vectors of an undeflected ray of light $\left(\overrightarrow{k_{i}}\right)$ and that same incident ray scattered at some angle $\theta, \overrightarrow{k_{s}}$ :

$$
\vec{q}=\overrightarrow{k_{i}}-\overrightarrow{k_{s}}
$$

Assuming elastic light scattering, the magnitudes of the scattered and incident wave vectors are equal, and can be written as:

$$
\left|\overrightarrow{k_{i}}\right|=\left|\overrightarrow{k_{s}}\right|=\frac{2 \pi}{\lambda}
$$

The magnitude of $\vec{q}$ can then be found by trigonometry,

$$
q=\frac{4 \pi}{\lambda} \sin \left(\frac{\theta}{2}\right)
$$

and the phase change at the detector of the scattered light is $e^{\imath \vec{q} r}$. The scattering wave vector is a useful parameter because its inverse, $q^{-1}$, has units of length and represents the length scale, or probe length, of the scattering experiment. The scattered intensity at the detector is a function of $q$ :

$$
I(q)=\left|\sum_{i=1}^{N} e^{\imath \vec{q} \vec{r}_{i}}\right|^{2}
$$

where $\vec{r}_{i}$ is the position vector of particle $i$. By inspection of Equation (2.12), one can see that if $q^{-1}$ changes significantly compared to $r$, then the scattering field will also change significantly. On the other hand, if $q^{-1}$ does not change much relative to $r$, the scattering field will not be altered dramatically. The structure factor represents 
the normalization of this intensity by $N^{-2}$,

$$
S(q)=\frac{1}{N^{2}}\left|\sum_{i=1}^{N} e^{\imath \vec{q} \vec{r}_{i}}\right|^{2}
$$

This means that the length $q^{-1}$ can then be compared to the radius of the scattering particle to determine which scattering regime is appropriate. For the case of fractal aggregates, the radius used here is the radius of gyration, given by Equation (1.2). The product $q R_{g}$ is then a non-dimensional parameter that determines what form of the structure factor should be used. There are three such regimes, the first being the power-law regime, valid for $q R_{g} \gg 1$, the second is the Guinier regime, valid for $q R_{g} \lesssim 1$, and the third is the Rayleigh regime, valid for $q R_{g} \ll 1$. The Rayleigh regime is then the small $q R_{g}$ limit of the Guinier regime. The corresponding structure factors are written as follows [Sorensen, 2001]:

$$
S(q)= \begin{cases}1 & q R_{g} \ll 1 \\ 1-\frac{1}{3} q^{2} R_{g}^{2} & q R_{g} \lesssim 1 \\ C\left(q R_{g}\right)^{-D_{f}} & q R_{g} \gg 1\end{cases}
$$

The factor $C$ in the power-law regime structure factor can take many forms, having been found by empirical correlation in several studies. These correlations are evaluated and compared by Sorensen [2001], who recommends a value of $C=1.0 \pm 0.05$ for aggregates with $1.7<D_{f}<2.2$. For a numerical implementation of this calculation, however, a more rigorous form of the structure function must be obtained, one capable of handling the transition between regimes without discontinuity. 


\subsubsection{Explicit Form of the Structure Factor}

The structure factor represents the spatial Fourier transform of the aggregate density auto-correlation function, which has the form

$$
g(r)=A r^{D_{f}-d} h(r / \xi)
$$

where $d$ is the spatial dimension, $A$ is a constant, $\xi$ is a characteristic length parameter, and $h(r / \xi)=e^{-(r / \xi)^{\beta}}$ is a cutoff function that describes the perimeter of the aggregate, where $\beta$ is the cutoff exponent. Sorensen et al. [1992a] suggest that the so-called Gaussian value of $\beta=2$ provides the best fit to the data, compared to the exponential value $(\beta=1)$ as well as those found by experiment [Hurd and Flower, 1988], by simulation [Lin et al., 1990, Mountain and Mulholland, 1988], and by empirical fits of $S(q)$ to data [Dobbins and Megaridis, 1991]. Using the Gaussian cutoff of $h(r / \xi)=$ $e^{-(r / \xi)^{2}}$ with Equation (2.15) and taking the Fourier transform, results in the structure factor having the form [Sorensen et al., 1992a]

$$
S(q)=e^{-\left(q R_{g}\right)^{2} / D_{f}}{ }_{1} F_{1}\left[\frac{3-D}{2}, \frac{3}{2}, \frac{\left(q R_{g}\right)^{2}}{D_{f}}\right]
$$

where ${ }_{1} F_{1}$ is the confluent hyper-geometric series, and the characteristic length is

$$
\xi^{2}=\frac{4}{D_{f}} R_{g}^{2}
$$

This explicit form of the structure function allows calculation of the structure function for all values of $q R_{g}$, asymptotically approaching the power law form at high $q R_{g}$, with the factor $C$ in Equation (2.14) described using the Gamma function, $\Gamma(z)=$ 
$\int_{0}^{\infty} t^{z-1} e^{-t} d t$, as

$$
C=D_{f}^{D_{f} / 2} \frac{\Gamma(3 / 2)}{\Gamma\left(\frac{3-D_{f}}{2}\right)}
$$

With both the scattering and absorption cross-sections defined for a single aggregate, we can apply them to some polydisperse population through an aggregate size distribution function.

\subsubsection{Polydisperse Populations and Probability Distributions}

In a polydisperse population of soot aggregates, the bulk scattering cross-section, comprised of the total of all aggregates per unit volume, is given by Equation (2.19). This bulk property is also known as the scattering coefficient, $K_{\text {sca }}$, which describes the fraction of light scattered per unit length traveled through the medium.

$$
\begin{aligned}
K_{s c a, \lambda} & =\int \frac{d \sigma_{s c a}^{p}}{d \Omega} \int_{1}^{\infty} n P(N ; x, u x) N^{2} S\left[q(\theta), R_{g}(N)\right] d N d \Omega \\
& =\iint \frac{\pi^{4} d_{p}^{6}}{4 \lambda^{4}} F(m)_{\lambda} \int_{1}^{\infty} n P(N ; x, u x) N^{2} S\left[q(\theta), R_{g}(N)\right] d N d(\cos \phi) d \theta
\end{aligned}
$$

For non-polarized incident light, the dependence on azimuth angle disappears, resulting in the following expression, [e.g. Köylü and Faeth, 1994a]

$$
K_{s c a, \lambda}=2 \pi \int_{0}^{\pi} \frac{\pi^{4} d_{p}^{6}}{4 \lambda^{4}} F(m)_{\lambda} \int_{1}^{\infty} n P(N ; x, u x) N^{2} S\left[q(\theta), R_{g}(N)\right] d N \frac{1+\cos ^{2}(\theta)}{2} \sin (\theta) d \theta
$$


Similarly, the bulk absorption cross-section is the absorption coefficient, $K_{a b s}$, given by Equation (2.22), and is equivalent to the summation of all contributing particles:

$$
\begin{aligned}
K_{a b s, \lambda} & =\sigma_{a b s, \lambda}^{p} \int n P(N ; x, u x) N d N \\
& =\frac{\pi^{2} d_{p}^{3}}{\lambda} E(m)_{\lambda} \int n P(N ; x, u x) N d N
\end{aligned}
$$

A bulk extinction coefficient, $K_{\text {ext }}$, can be defined in a similar way to Equation (2.2), as

$$
K_{e x t}=K_{a b s}+K_{s c a}
$$

The volume fraction, $f_{v}$, of the particles can be found by finding the volume of a single primary particle $\left(\frac{\pi}{6} d_{p}^{3}\right)$, multiplied by the number of primary particles per unit volume, which requires integration over the probability distribution:

$$
f_{v}=\int \frac{\pi d_{p}^{3}}{6} n P(N ; x, u x) d N
$$

This can be substituted into Equation (2.23) to produce a simpler form of the absorption coefficient,

$$
K_{a b s, \lambda}=\frac{6 \pi E(m)_{\lambda}}{\lambda} f_{v}
$$

This surprisingly simple relation is important because it shows that the absorption coefficient is proportional to the soot volume fraction, given the wavelength of light used and the absorption properties of soot. For the common experimental measurement of total extinction, the absorption coefficient can be substituted out using Equation 
(2.24) to give

$$
\begin{aligned}
K_{e x t, \lambda}-K_{s c a, \lambda} & =\frac{6 \pi E(m)_{\lambda}}{\lambda} f_{v} \\
\text { or } K_{e x t, \lambda} & =\frac{6 \pi\left(1+\rho_{s a}\right) E(m)_{\lambda}}{\lambda} f_{v}
\end{aligned}
$$

where $\rho_{s a, \lambda}=K_{s c a, \lambda} / K_{a b s, \lambda}$ is the ratio of scattering to absorption coefficients. Equation (2.28) can be used as the governing equation for optical extinction measurements of particles within the Rayleigh limit. To support the goal of the present work, this can be rewritten to solve for $E(m)_{\lambda}$ as:

$$
E(m)_{\lambda}=\frac{K_{e x t, \lambda} \lambda}{6 \pi\left(1+\rho_{s a, \lambda}\right) f_{v}}
$$

Thus, to determine $E(m)_{\lambda}$ we require measurements of the spectrally-resolved extinction coefficient, the wavelength of light, the soot volume fraction, and the spectrallyresolved ratio of scatter to absorption.

\subsubsection{Scatter-to-Absorption Ratio}

As mentioned above, the goal of this research is to determine $E(m)_{\lambda}$ using Equation (2.29). The term $\rho_{s a, \lambda}$ is simply a ratio of the scattering to absorption coefficients, given above:

$$
\begin{aligned}
\rho_{s a, \lambda} & =\frac{2 \pi \int_{0}^{\pi} \frac{\pi^{4} d_{p}^{6}}{4 \lambda^{4}} F(m)_{\lambda}\left[\int_{1}^{\infty} n P(N ; x, u x) N^{2} S\left[q(\theta), R_{g}(N)\right] d N\right] \frac{1+\cos ^{2}(\theta)}{2} \sin (\theta) d \theta}{\frac{\pi^{2} d_{p}^{3}}{\lambda} E(m)_{\lambda} \int n P(N ; x, u x) N d N} \\
& =\frac{\pi^{3} d_{p}^{3}}{2 \lambda^{3}} \frac{F(m)_{\lambda}}{E(m)_{\lambda}} \frac{\int_{0}^{\pi} \int_{1}^{\infty} P(N ; x, u x) N^{2} S\left[q(\theta), R_{g}(N)\right] d N \frac{1+\cos ^{2}(\theta)}{2} \sin (\theta) d \theta}{\int P(N ; x, u x) N d N}(2.31)
\end{aligned}
$$


Some interesting features of Equation (2.31) are that $F(m)_{\lambda}$ and $E(m)_{\lambda}$ appear only as a ratio, and that the number concentration of aggregates cancels out. Thus $\rho_{s a, \lambda}$ is a function of the soot morphology (aggregate size probability distribution and its parameters, $P(N ; x, u x)$, the fractal parameters $D_{f}$ and $k_{g}$, and the primary particle diameter $d_{p}$ ), the wavelength of light in question, $\lambda$, and the ratio of scattering to absorption refractive index functions, $F(m)_{\lambda} / E(m)_{\lambda}$.

\subsubsection{Evaluation of RDG-PFA Theory}

The performance of RDG-PFA theory has been evaluated by several groups, finding encouraging agreement between RDG-PFA predictions and experimental data [e.g. Köylü and Faeth, 1994a,b, Sorensen et al., 1992b], as well as with the more rigorous computational models discussed above [e.g. Ku and Shim, 1992, Van-Hulle et al., 2002].

The volume integral equation formulation for scattering (VIEF) is considered the most elegant and theoretically sound numerical method, accounting for multiple scattering and self-interaction without the need for assumptions. Farias et al. [1996] compared the predictions of the VIEF and RDG-PFA approximations on a multitude of numerically-simulated mass fractal aggregates with widely varied parameters, encompassing the ranges typically found of in-flame soot. Results were presented in the $x_{p}|m-1|$ domain, which represents the phase difference between a ray of light passing through the particle and that of a ray passing through the surrounding medium. The RDG-PFA approximation is taken to be valid for aggregates with $2 x_{p}|m-1| \ll 1$. The results of the comparison show that the RDG-PFA approximation is:

- insensitive to morphology, with the effect of the fractal dimension found to be particularly insignificant, and 
- valid to within $10 \%$, given that $|m-1| \approx 1$ and that the primary particles are within the Rayleigh limit $\left(x_{p} \ll 1\right)$.

It is, however, worth noting that this study considered aggregates with $N=16-256$, and that the $10 \%$ error contours tend to shrink with increasing $N$. In the current case of post-flame soot, a portion of aggregates have values of $N$ that significantly exceed this range. More recently, however, Liu and Snelling [2008] have shown that the results of the RDG approximation are within $10 \%$ of the generalized multi-sphere Mie solution for simulated monodisperse aggregates even for large values of $N$. Thus, for the purpose of this research it can be concluded that the RDG-PFA approximation is generally good to approximately $10 \%$. 


\section{Chapter 3}

\section{Experimental Setup}

The objective of this research was to use Equation (2.29) to determine values of the spectrally-resolved soot absorptive refractive index function, $E(m)_{\lambda}$. This required determination of three separate experimental quantities: the soot volume fraction $f_{v}$, the scatter-to-absorption ratio, $\rho_{s a, \lambda}$, and the spectral extinction coefficient $K_{e x t, \lambda}$. Soot volume fraction was determined by gravimetric analysis, as described in Section 3.2. Determination of the scatter-to-absorption ratio first required knowledge of the soot morphology, which was found by electron microscopy as discussed in Section 3.3. This morphological information $\left(d_{p}, N, D_{f}, k_{g}\right.$, and aggregate size distribution) was then used to support numerical calculation of $\rho_{s a, \lambda}$ according to Equation (2.31), as discussed in Section 3.4. Finally, $K_{e x t, \lambda}$ was determined by a spectral line-of-sight attenuation measurement described in Section 3.5. However, the soot under study must first be generated, which is the topic of the following section. 


\subsection{Inverted Flame Soot Generator}

An inverted co-flow diffusion flame burner was developed as a means of generating soot in a wide range of sizes and concentrations, using the work of Stipe et al. [2005] as a starting point. The repeatability of the burner was verified using a scanning mobility particle sizer (SMPS), discussed in Appendix A. The layout of the burner and a cross-sectional view of the burner head can be seen in Figure 3.1. This burner was designed to be modular for ease of assembly and modification, and consists of three main sections: the burner head, the combustion chamber, and the exhaust tube.

The aluminum head of the burner takes in the fuel and co-flow air and passes them to the combustion chamber. The fuel enters the head through Station 1 in Figure 3.1(a) and continues through to the combustion chamber, as shown by the vertical tube in Figure 3.1(b). Co-flow air enters a plenum in the head through Station 2, where it is distributed and straightened by glass beads and a $13 \mathrm{~mm}$ section of sintered metal foam before it enters the combustion chamber. Also shown in Figure 3.1(b) is a bluff-body obstruction on the nozzle of the fuel tube which stabilizes the flame [Stipe et al., 2005]. Acting as this bluff. body is an unmodified 3/4" union tube fitting (SwageLok, SS-1210-6), compression-fitted onto the end of the fuel tube.

The combustion chamber consists of mating flanges and a quartz tube, which is approximately $480 \mathrm{~mm}$ in exposed length, and through which the operator can visually monitor the flame. The quartz tube is sealed to the upper flange by means of a high-temperature (Viton) o-ring, seen in Figure 3.1(b), and both ends are seated on a layer of ceramic cloth gasket to prevent direct contact between the quartz tube and the aluminum flanges. Four vertical struts attach the tube to the upper and lower flanges, which in turn connect the combustion chamber to the head and the exhaust tube, respectively. The assembled sections can be seen in both the model and the 


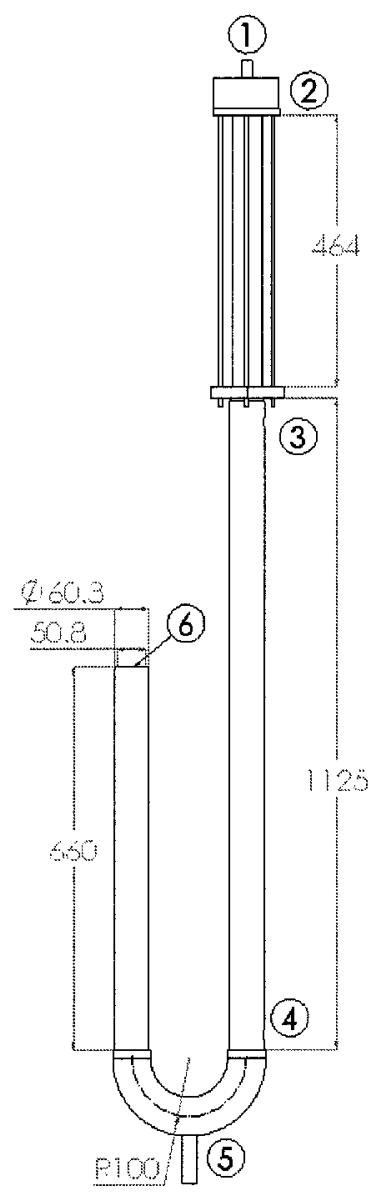

(a)

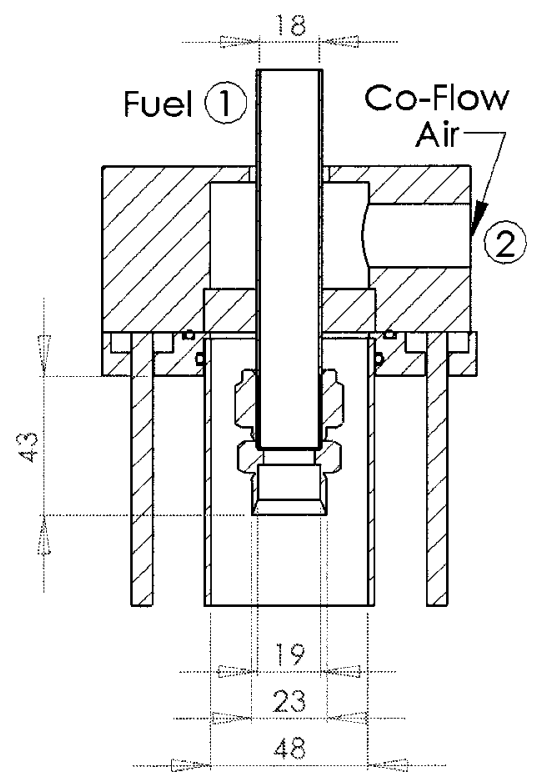

(b)

Figure 3.1: (a) Layout of the burner system and (b) cross-section of the burner head. Dimensions are in millimeters. 


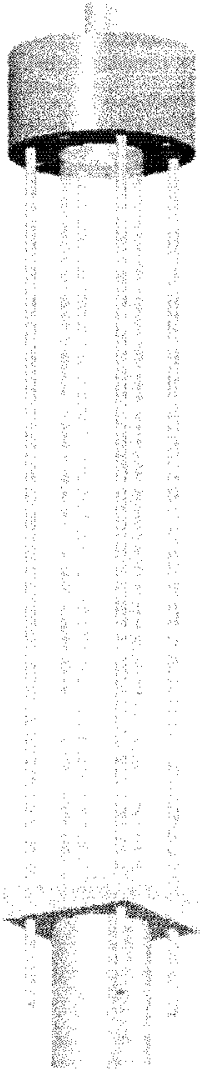

(a)

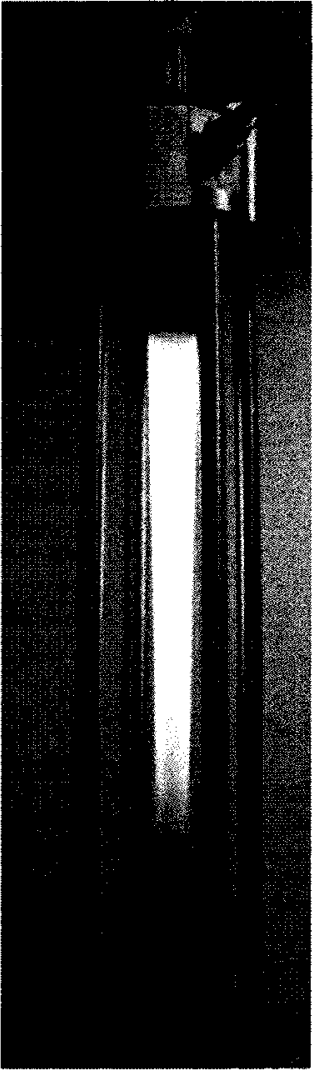

(b)

Figure 3.2: The combustion chamber of the inverted burner in (a) a solid model and (b) a photograph

photo of Figure 3.2 .

The burner exhaust tube consists of a stainless-steel pipe in which the flow mixes and cools, followed by a section of ABS tubing that turns and exhausts the flow. The steel tube is approximately $1150 \mathrm{~mm}$ in length and features a port near each end. The top port, Station 3 in Figure 3.1(a), serves as an inlet for dilution air flow. The lower port (Station 4) is an auxiliary sampling port. The ABS tube then turns the exhaust stream through $180^{\circ}$ and drains any condensed water from Station 5 in Figure 3.1(a). Note that the drain outlet is kept below water level to prevent mixing of the exhaust gas and open air, and that condensation is only a startup issue since temperatures 


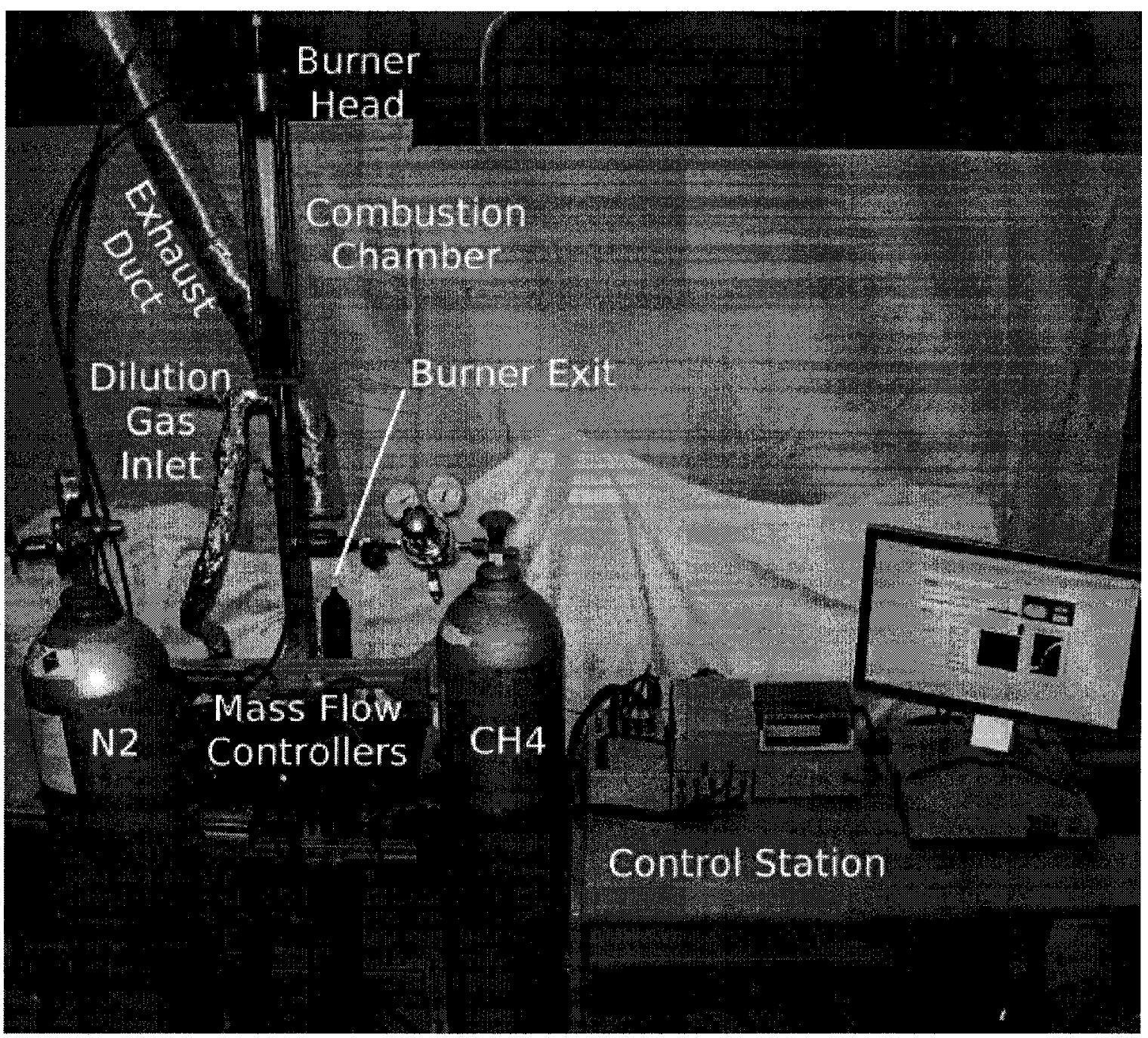

Figure 3.3: Photograph of the burner setup

are high enough to avoid condensation under normal operating conditions. Finally, the ABS tube carries the flow upward and exhausts it at a convenient height (Station 6), where soot diagnostics are performed. A photograph of the entire setup can be seen in Figure 3.3.

Flow rates of all gases (fuel, co-flow air, and diluent) are controlled by thermal mass flow controllers (Brooks, SMART series), the input and output of which are managed by a LabVIEW virtual instrument. 


\subsubsection{Burner Conditions}

A total of twelve burner conditions were studied, with three main variables of interest: dilution ratio, dilution temperature, and dilution gas. Three different dilution rates were sampled for three different dilution air temperatures each. One of those conditions was also selected to be run with nitrogen as the diluent rather than air to investigate post-flame oxidation effects. Finally, a slightly globally-rich (underventilated) condition was run with both air and nitrogen diluents, so that no excess oxygen was present in the exhaust stream and thus no post-flame soot oxidation could occur. The twelve burner conditions are summarized in Table 3.1. Global equivalence ratios were determined assuming complete (ideal) combustion of $\mathrm{CH}_{4}$ with air consisting of $21 \%$ oxygen, according to $\phi=2 Q_{\text {fuel }} / 0.21 Q_{a i r}$, and dilution ratio $(D R)$ was defined as the volumetric ratio of dilution gas to combustion products, which for methane can be expressed as $D R=Q_{d i l} /\left(Q_{f u e l}+Q_{a i r}\right)$. The labelling scheme is somewhat arbitrary, though it does carry some meaning: series C, D, and E indicate the varying dilution rate, and each numerical suffix refers to a different dilution inlet temperature (e.g. C1 indicates 50 SLPM of dilution air at 100 degrees, D2 indicates 40 SLPM dilution at 60 degrees, etc.). The $\mathrm{B}$ series is the underventilated condition, the first with air as the diluent and the second with nitrogen. A1 corresponds to condition C3, only with nitrogen as the diluent.

\subsection{Gravimetric Analysis}

Gravimetric analysis was used to obtain a measurement of soot concentration that was independent of optical properties. To do this, soot was collected on a filter from some known volume of exhaust gas, $V$, and weighed to determine the mass of soot, $m_{s}$. Assuming a soot density, $\rho_{s}$, from literature, the soot volume could then be found. 
Table 3.1: Summary of Burner Conditions

\begin{tabular}{|c|c|c|c|c|c|c|c|}
\hline Label & $\begin{array}{c}Q_{\text {fuel }} \\
{[\text { SLPM] }]}\end{array}$ & $\begin{array}{c}Q_{\text {air }} \\
{[\text { SLPM }]}\end{array}$ & $\begin{array}{c}Q_{\text {dil }} \\
{[\text { SLPM }]}\end{array}$ & $\begin{array}{c}\text { Dil. gas } \\
\left(N_{2} / \text { air }\right)\end{array}$ & $\begin{array}{c}T_{\text {dil }} \\
{\left[{ }^{\circ} \mathrm{C}\right]}\end{array}$ & $\begin{array}{c}\phi \\
\text { (global) }\end{array}$ & $\begin{array}{c}D R \\
(\text { vol. })\end{array}$ \\
\hline \hline A1 & 1.2 & 15 & 50 & $N_{2}$ & 25 & 0.76 & 3.1 \\
\hline B1 & 1.4 & 13 & 50 & air & 25 & 1.03 & 3.5 \\
\hline B2 & 1.4 & 13 & 50 & $N_{2}$ & 25 & 1.03 & 3.5 \\
\hline C1 & 1.2 & 15 & 50 & air & 100 & 0.76 & 3.1 \\
\hline C2 & 1.2 & 15 & 50 & air & 60 & 0.76 & 3.1 \\
\hline C3 & 1.2 & 15 & 50 & air & 25 & 0.76 & 3.1 \\
\hline D1 & 1.2 & 15 & 40 & air & 100 & 0.76 & 2.5 \\
\hline D2 & 1.2 & 15 & 40 & air & 60 & 0.76 & 2.5 \\
\hline D3 & 1.2 & 15 & 40 & air & 25 & 0.76 & 2.5 \\
\hline E1 & 1.2 & 15 & 30 & air & 100 & 0.76 & 1.9 \\
\hline E2 & 1.2 & 15 & 30 & air & 60 & 0.76 & 1.9 \\
\hline E3 & 1.2 & 15 & 30 & air & 25 & 0.76 & 1.9 \\
\hline
\end{tabular}

The soot volume fraction was then simply the ratio of the sampled soot volume to the sampled gas volume, corrected for temperature change if necessary; mathematically, this is

$$
f_{v}=\frac{m_{s}}{\rho_{s} V} \frac{T_{s}}{T_{\infty}}
$$

where $T_{s}$ is the soot/exhaust gas temperature at the burner exit and $T_{\infty}$ is the gas temperature at the point of measurement (ambient).

Soot was sampled from the exhaust plume using an isokinetic sampling system, described below, which matches the velocity of the gas being sampled with that of the surrounding exhaust stream. The exhaust velocity profile was measured by a pitot-tube traversed at the outlet, and average velocity found by continuity from the total input flow rate, corrected for temperature. Membrane filters (Whatman, Anodisc $25 \mathrm{~mm}$ diameter) with a pore size of $0.02 \mu \mathrm{m}$ were used for soot collection, and weight measurements were made with an electronic micro-balance (Mettler Toledo, UMX2) both before and after soot was collected. The volume of exhaust gas from 
which the soot was collected was measured with a thermal mass flow meter (Brooks, SMART series), and the exhaust gas temperature was monitored with a thermocouple. These data were acquired by the LabVIEW virtual instrument at a rate of $10 \mathrm{~Hz}$ (hardware-timed). In addition to the uncertainties related to these measurements, there are several sources of uncertainty in the procedure, including vibration of the balance, electrostatic charges, the mass of contaminants retained in the filter along with the soot (e.g. moisture), and buoyancy effects due to fluctuations in the temperature and humidity of the filter conditioning environment [Taylor, 2006]. Balance vibration was addressed by isolating the balance with an air suspension isolation table (Kinetic Systems Inc., Vibraplane). It has been shown [e.g. Tsai et al., 2002, Taylor, 2006] that the accuracy of the weight measurement can be improved significantly through use of controlled environmental conditions to reduce buoyancy and moisture effects, and improved further by neutralizing electrostatic charges on the samples prior to weighing. The filter material used in the present work (alumina) was not one of the materials tested; however, both measures were nonetheless taken: electrostatic charges were reduced by neutralizing the samples with an alpha-radiation neutralizer (Staticmaster, 2U500), and moisture and buoyancy issues were addressed by conditioning the samples in a climate-controlled clean room at $25^{\circ} \mathrm{C}$ and $50 \%$ relative humidity for a minimum of 24 hours prior to weighing.

A nominal value of forty samples were made for each of the twelve conditions, although a small number were discarded due to flaws, breakage, or contamination. Included in this number were four control filters per condition, which were handled in the same way as the samples, only without collecting any soot. The controls were used to detect any drifting of the balance, and for each condition the recorded masses are adjusted by the mean deviation of the controls, which were typically less than one percent. For each condition, ten of the forty samples were taken for each of four 
durations, $60,80,100$, and 120 seconds, to ensure linearity of the measured soot volume fraction with sample time. Repeatability tests were performed to measure the sample-by-sample variability in the balance measurements, which was found to be significantly greater than the quoted precision of the balance. A complete uncertainty analysis is presented alongside the results in Chapter 4.

\subsubsection{Isokinetic Sampling System}

An isokinetic sampling system was designed to extract a portion of the exhaust stream while matching the sample gas velocity to the surrounding exhaust gas velocity, to minimize any potential bias in the soot collection from particle momentum effects. The exhaust gas velocity profile was measured by pitot tube traverse, and gas velocity was estimated using the total volumetric input to the burner and correcting for temperature at the burner exit (measured by a thermocouple) via the ideal gas law. The membrane filters used to collect soot produce a substantial pressure drop, limiting the achievable flow rate through the filter. To compensate for this, a cubic-contoured diffuser was designed to decrease the cross-sectional area and thus, by continuity, increase the sampling velocity. The system consists of the following components, shown in Figure 3.4: the diffuser, the filter holder, the mass flow controller, the vacuum pump, and the control and measurement system - the same LabVIEW virtual instrument that controls the burner. Filters were mounted by removing the threaded diffuser and placing the clean filter on the holder. The diffuser was sealed to the filter holder by means of an o-ring, which compressed against the outer edge of the filter support ring and the body of the filter holder. 


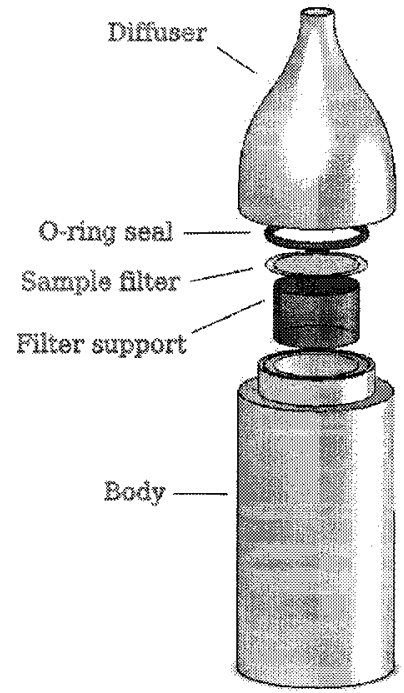

(a)

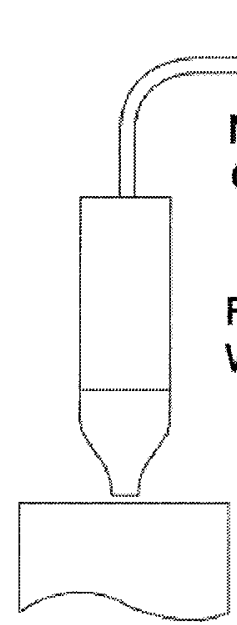

Burner Exhaust

Figure 3.4: (a) Solid model rendering and (b) schematic of the isokinetic sampling system

\subsection{Soot Characterization}

The extinction measurements needed to be corrected for light scatter, which is heavily dependent on the size of the particles. Thus, it was important to have an accurate estimate of the soot morphology ( $d_{p}$, fractal properties, size distribution) to correct for scatter when making extinction measurements. As discussed in Section 1.2.2, thermophoretic sampling and analysis by transmission electron microscopy (TS-TEM) is the standard method of soot extraction for imaging, but the advantage of non-biased aggregate collection of this method relies on a large temperature gradient between the gas and the probe, which does not exist in the present case of a cooled exhaust stream. Thus, in this case, TS-TEM sampling is only suitable for measurement of the primary particle diameter, which is independent of aggregate size, and is unsuitable for determining the aggregate size distribution. Instead, soot was collected on filters 
and imaged using a scanning electron microscope (SEM). However, the nature of SEM requires the sample to be electrically conductive, so in cases such as this one where the sample itself is not conductive, a thin coating (e.g. $10 \mathrm{~nm}$ ) of conductive material (e.g. gold) must be applied. This introduces some additional uncertainty in the projected area measurements taken from these images: for a soot aggregate several hundreds of nanometers in diameter, the error introduced by the conductive layer is small. However for a primary particle only $30 \mathrm{~nm}$ in diameter, this error could be significant. Therefore, TEM imaging is used to obtain $d_{p}$, which is approximately uniform [e.g. Köylü et al., 1995a] and therefore not affected by collection bias.

\subsubsection{TEM sampling}

Sampling was performed using 3-mm carbon-coated copper grids (Electron Microscopy Sciences, Cat. no. 71150), manually held in the exhaust stream for approximately one minute. Samples were imaged with a TEM (Philips, CM20) at a magnification of $18000 \mathrm{X}$, and 80-100 measurements of $d_{p}$ were made for each sampling condition, using the commercial Image Pro Plus 4.5.1 software (Media Cybernetics). The results are summarized in Table 4.3, and a sample TEM micrograph can be seen in Figure 3.5. Measurements were generally made on thinner aggregate branches, so that individual primary particles were more easily distinguishable.

\subsubsection{SEM sampling}

As discussed in Section 1.2.2, for the conditions being studied, the lack of a strong temperature gradient between the exhaust gas and a thermophoretic TEM sampling probe calls into question the un-biased nature of TS-TEM sampling for measuring aggregate size distributions. These data were instead determined through filter sampling 


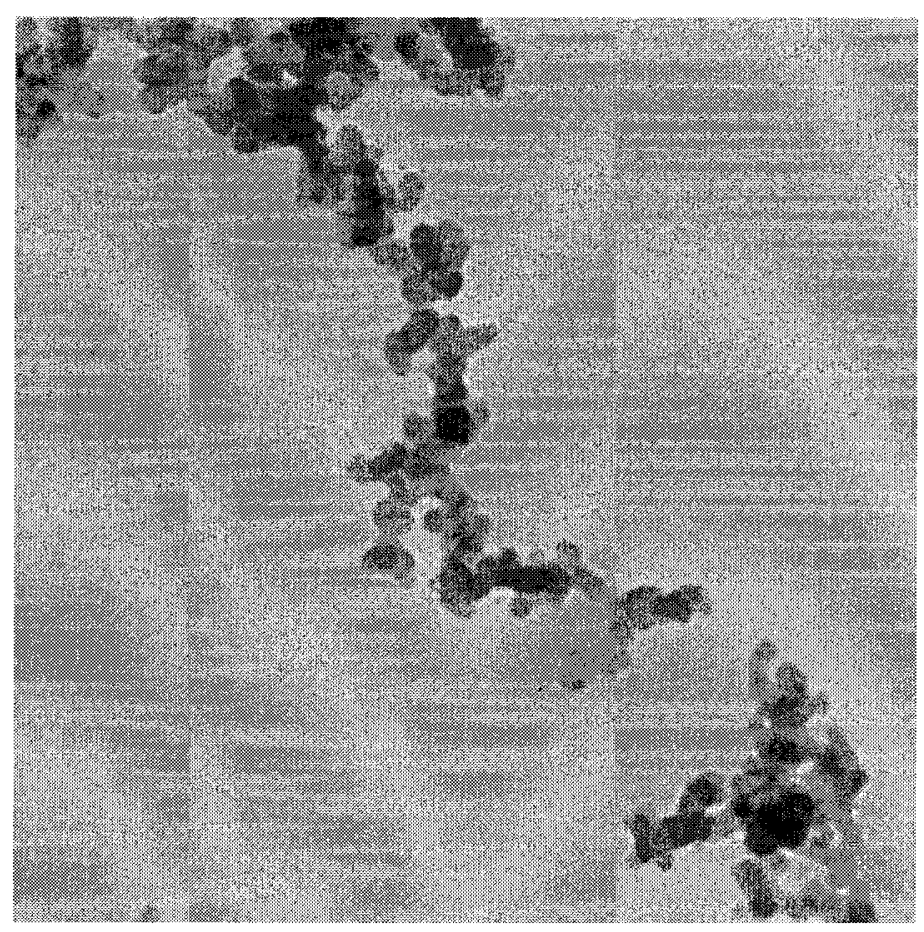

Figure 3.5: Typical TEM image used for $d_{p}$ measurement

of the exhaust plume and imaging via SEM (JEOL, 840A). The sampling apparatus is identical to that described above for the gravimetric analysis, only the sampling time was reduced to approximately 1 second (including pump ramp up and ramp down time), which was found by trial and error to yield an appropriate surface coverage (approximately 10\% and consistent with that used by Köylü and Faeth [1992]). The samples were coated with gold by sputter deposition with a thickness of $10 \mathrm{~nm}$. The SEM microphotographs were analyzed according to the procedure discussed in the following section, using the commercial Image-Pro Plus 4.5.1 software from Media Cybernetics. Aggregate characterization largely followed the method of Brasil et al. [1999]. 


\subsubsection{SEM Image Analysis}

The analysis of SEM images was considerably more difficult than for the standard TEM images, due primarily to the added complexity of separating aggregates from the background features. This is generally easy to do by eye, though it has proven difficult to do by algorithm. The SEM images capture an element of the three-dimensional structure of the aggregates, which shows up as variations in the brightness, or intensity, with the brightest being the furthest from the filter surface. A typical aggregate as seen on an SEM image is shown in Figure 3.8.

The object counting algorithms in Image Pro Plus separate objects from the background using a threshold filter, which binarizes an image (sets to either black or white for a grayscale image) according to a chosen intensity threshold. As seen in Figure 3.8 , the difficulty arises from portions of the aggregate that have a lower intensity than some portions of the background. Thresholding the unmodified image would introduce extra area from the background (false positives) in some regions, while subtracting legitimate aggregate area (false negatives) in other regions. An example of this is given in Figure 3.9 below. Simply relying on thresholding to separate objects is clearly inadequate.

There exist myriad processing filters designed to deal with such issues. These filters are grouped in the software under the following categories: enhancement, edge, and morphological. Enhancement, or convolution, filters perform operations on local neighbourhoods of pixels, called kernels. Edge detection is done by finding significant local changes in pixel intensity, detected through some measurement of the intensity gradient; the various edge detection algorithms differ in the way this gradient is calculated [Acharya and Ray, 2005]. Unfortunately, the background intensity variations in the SEM images are such that edge detecting algorithms can not distinguish be- 
tween aggregate edges and filter pore edges, limiting their applicability in this work. Morphological filters identify regions within images, and perform operations on those regions. The primary goal of many morphological filters is to simplify the image for easier segmentation into regions [Salembier, 1994].

A useful sequence of image filters was found through extensive trial-and error testing, the goals being to even out the background and reduce the intensity variation in the aggregates such that the software could accurately separate the aggregate from the filter background, while minimizing any distortion of the aggregate shape (and hence maximizing the accuracy of the measurement). The following sequence of filters was applied to all processed images: flatten (dark background, 40-pixel features), median ( $7 \times 7$ pixel square kernel, 2 passes), median (5x5 pixel square kernel, 2 passes), median (3x3 pixel square kernel, 2 passes), and close (morphological, 11x11 pixel octagonal kernel, 1 pass). These three filters, all built into the software, are discussed briefly below.

The flatten filter removes intensity variations in the background of the image, its argument being the size in pixels of the objects in the foreground. Essentially this is a background normalization filter, which was useful in reducing background intensity gradients. This can be seen in Figure 3.6, where the left-to-right intensity gradient that exists in the raw image (a) is removed from the processed image (b). There are two arguments to the function: the first is whether the background is darker or lighter than the objects; and the second is the approximate size (in pixels) of the objects themselves. Figure 3.6(b) shows that background intensity fluctuations that are on the same size order as the objects (seen as a wavy texture) are not removed.

The median filter is a non-linear image enhancement filter that replaces the input pixel with the median of the pixels contained in the kernel. The kernel is an appropriately-sized array of neighbouring pixels, the number of which is typically odd 


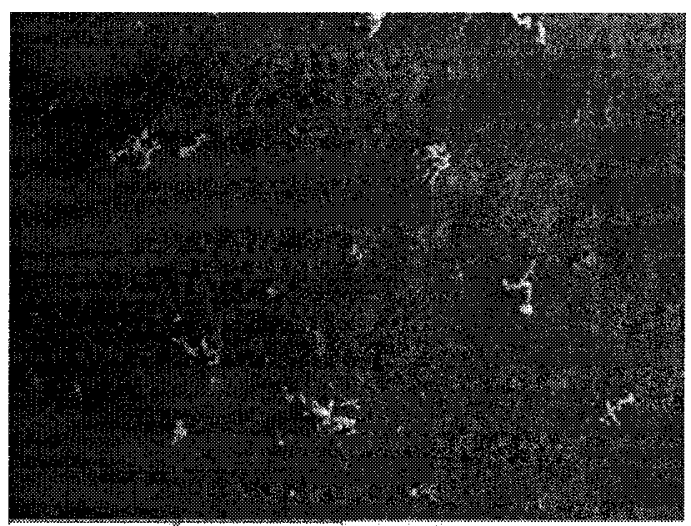

(a)

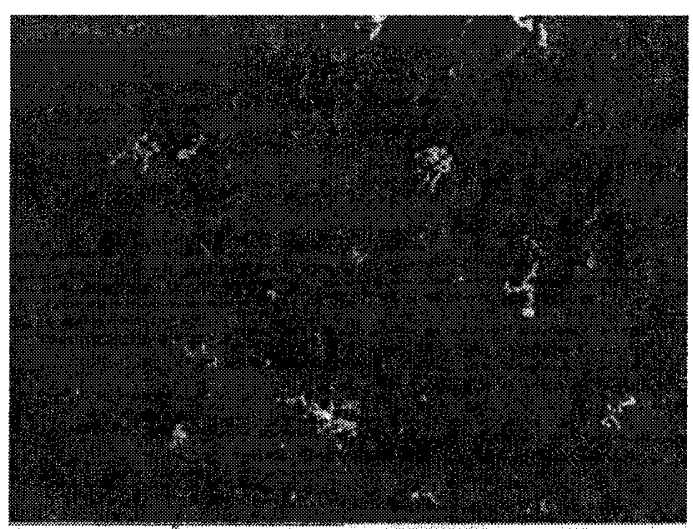

(b)

Figure 3.6: SEM image (a) before and (b) after application of the flatten filter

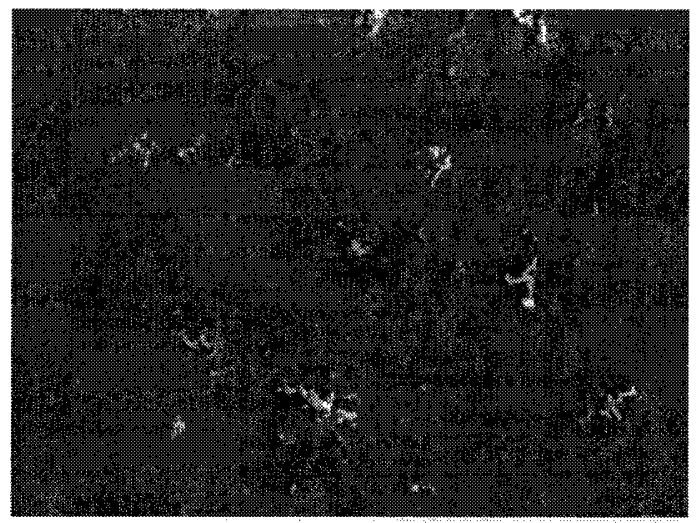

Figure 3.7: SEM image after application of the median filters

so that the median is a well-defined value corresponding to an individual pixel. The median filter is useful in removing noise while preserving spatial resolution [Acharya and Ray, 2005]. The arguments to this filter are the kernel size and shape, and number of passes. Figure 3.7 shows the result of the median filtering applied to the previous SEM image.

The close filter is a morphological filter that simplifies the signal by removing the dark (background) components that do not fit within the object [Salembier, 1994]. The result is that protrusions are enlarged and gaps between neighbouring objects are filled, connecting regions that are close together [Media Cybernetics, 2002]. The 


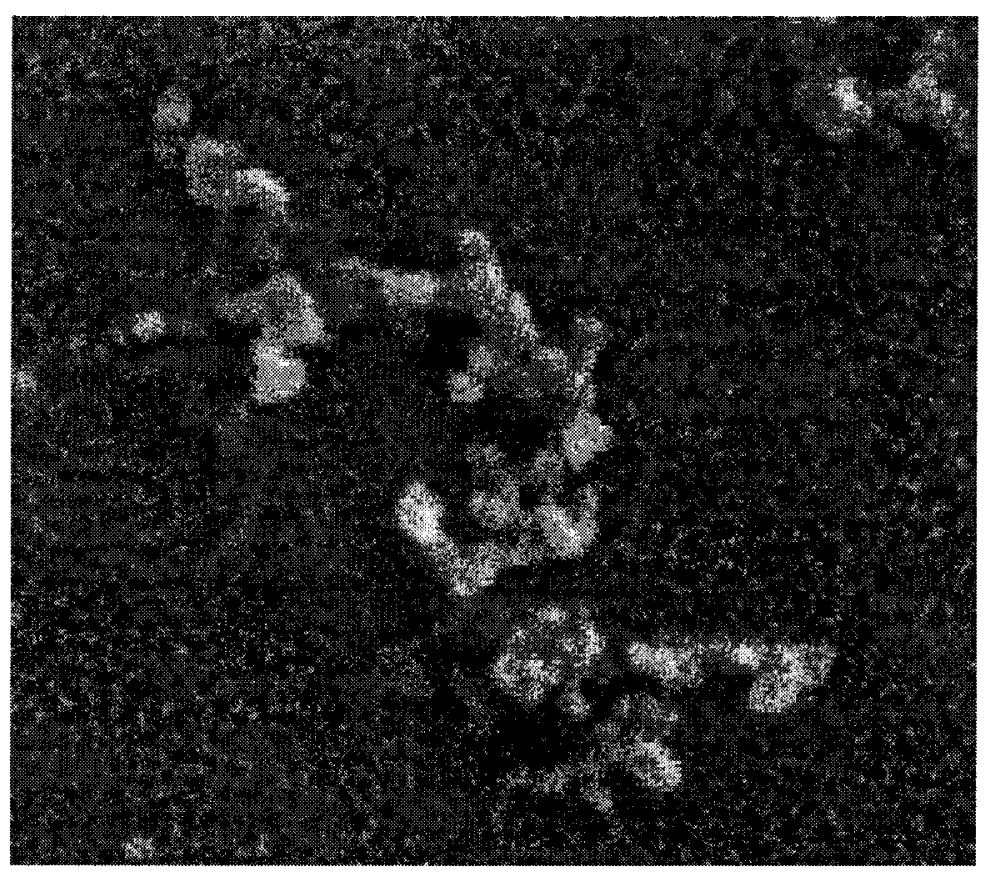

Figure 3.8: Aggregate in original SEM micrograph image

arguments are kernel size and shape, as well as the number of passes to perform.

The result of this processing is shown in Figure 3.10. For comparison with the unfiltered aggregate, Figure 3.11 shows an aggregate as measured overlaid on its unfiltered image. It is difficult to quantify the accuracy of this measurement, though it appears good according to manual inspection. The competing effects of added area from the gold coating and lost area from dark portions of the aggregate cancel each other out to some extent as well. This uncertainty is not accounted for directly, but is rolled in with the estimated uncertainty of the overall analysis, presented in Section 4.3 .

The object counting function in the software was then applied to the processed images. As mentioned above, this procedure relies on thresholding to separate objects from the background. The chosen intensity threshold was adjusted manually for each image to maximize accuracy, and object filters were applied to automatically 


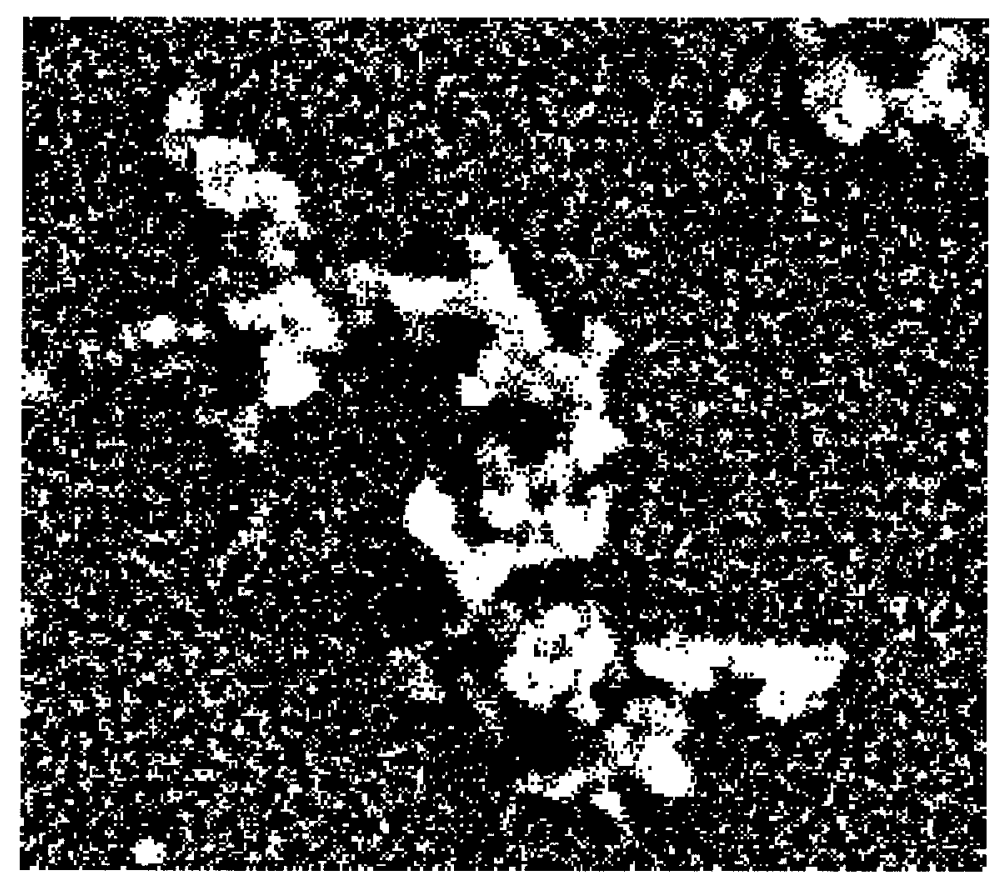

Figure 3.9: Result of thresholding an unprocessed SEM micrograph

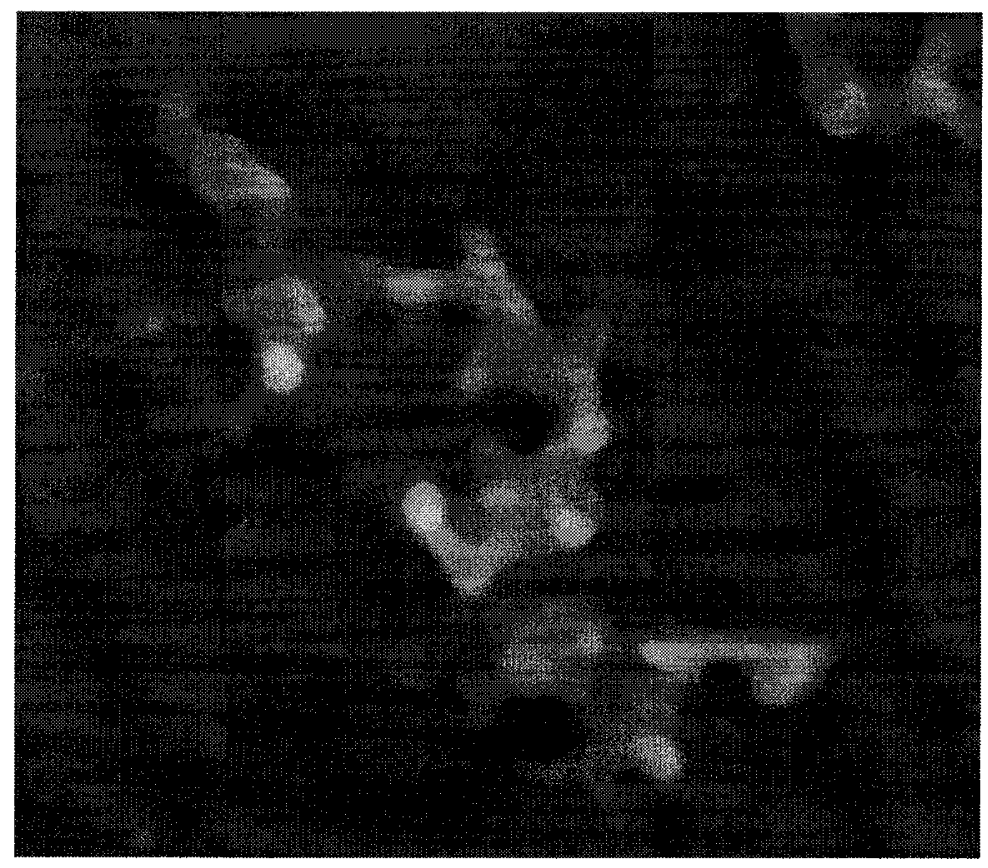

Figure 3.10: Aggregate in processed SEM micrograph 


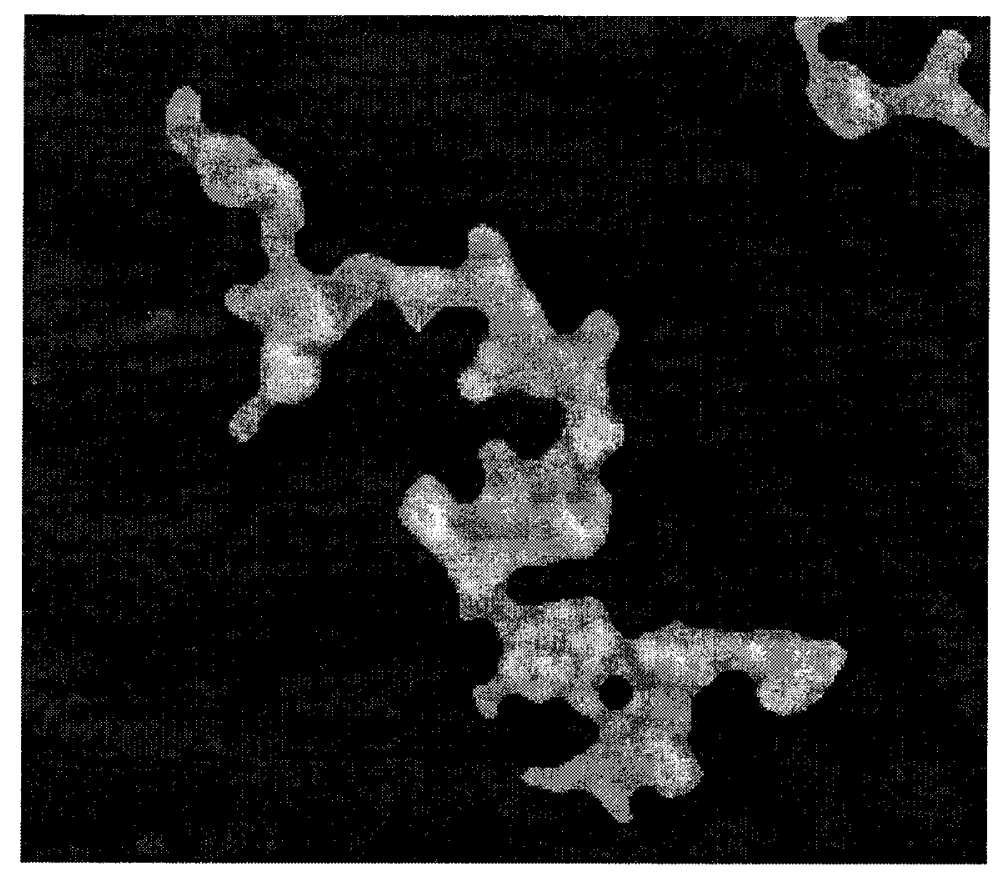

Figure 3.11: Aggregate as measured overlaid on original

discard non-soot objects (for example, objects with an area smaller than that of a single primary particle). In addition, each processed image with counted aggregates was manually inspected, and data for misrepresented particles (for example, a large aggregate being counted as several smaller aggregates) was discarded. An example of such a misrepresented aggregate is given in Figure 3.12. A statistically-significant number of aggregates (550-850) were measured for each condition in an effort to reduce uncertainty. The key dimensions measured in this analysis were the aggregate projected area and the maximum calliper length, also known as the Feret diameter. These measurements were used to determine key morphological parameters, as discussed in the following section. 


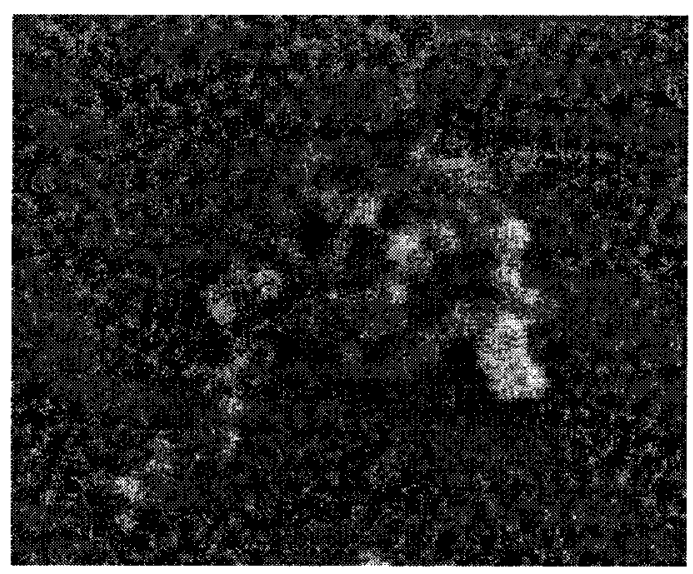

(a)

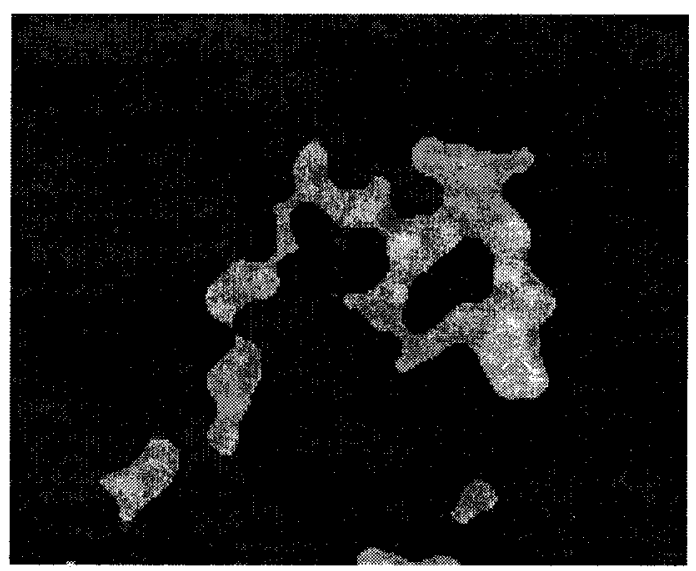

(b)

Figure 3.12: Misrepresented aggregate in (a) original micrograph, and (b) overlaid image

\subsubsection{Soot Aggregate Characterization}

As discussed in Section 1.1, soot aggregates can be characterized as mass fractals, where the number of primary particles per aggregate, $N$, can be related to the radius of gyration of the aggregate, $R_{g}$, by Equation (1.1). The method used to characterize our soot aggregates is based on the "recipe" outlined by Brasil et al. [1999], using the projected area method to determine the fractal parameters. Figure 3.13 from [Brasil et al., 1999] depicts the experimental measurements from the projected area and the three-dimensional properties that can be inferred (note that not all of these quantities were used in the present work).

The first step was to estimate $N$ using the ratio of projected areas of the aggregate and primary particles, as in the following relation:

$$
N=k_{a}\left(\frac{A_{a}}{A_{p}}\right)^{\alpha_{a}}
$$

where $A_{a}$ and $A_{p}$ are the measured projected areas of the aggregate and primary 
particle, respectively, and $k_{a}$ and $\alpha_{a}$ are empirical constants. The values of $k_{a}=1.16$ and $\alpha_{a}=1.10$ were used in this analysis, given by Köylü et al. [1995a] and found by analyzing random projections of simulated, soot-like fractal aggregates.

With $N$ estimated, the next step was to determine the fractal dimension. The following equation, again suggested by Köylü et al. [1995a], was used to fit the data,

$$
N=k_{L}\left(\frac{L}{d_{p}}\right)^{D_{f}}
$$

where $L$ is the measured maximum Feret diameter (maximum caliper length), $d_{p}$ is the primary particle diameter, $D_{f}$ is the fractal dimension and $k_{L}$ is a correlation prefactor. On a plot of $N$ versus $L / d_{p}$, as seen in Figure 3.14, the slope of the best-fit line returns the exponent and the intercept returns the prefactor. Finally, to convert this correlation prefactor to the desired fractal prefactor, the following relation was used [Brasil et al., 1999, Köylü et al., 1995b]:

$$
k_{g}=k_{L}\left(\frac{D_{f}+2}{D_{f}}\right)^{D_{f} / 2}
$$

With that, the fractal description of the aggregates is complete. The remaining soot characterization parameters to be found are those that describe the aggregate size distribution, which are discussed in the following section.

\subsubsection{Fitting Data to Probability Distributions}

As discussed in Section 1.1.2, both log-normal and self-preserving distributions of soot aggregate sizes were considered in this analysis. Normalized histograms, with log-based bin widths, were plotted for the aggregate size data from the SEM analysis discussed above. The fitting functions corresponded to Equations (1.5) and (1.9), 


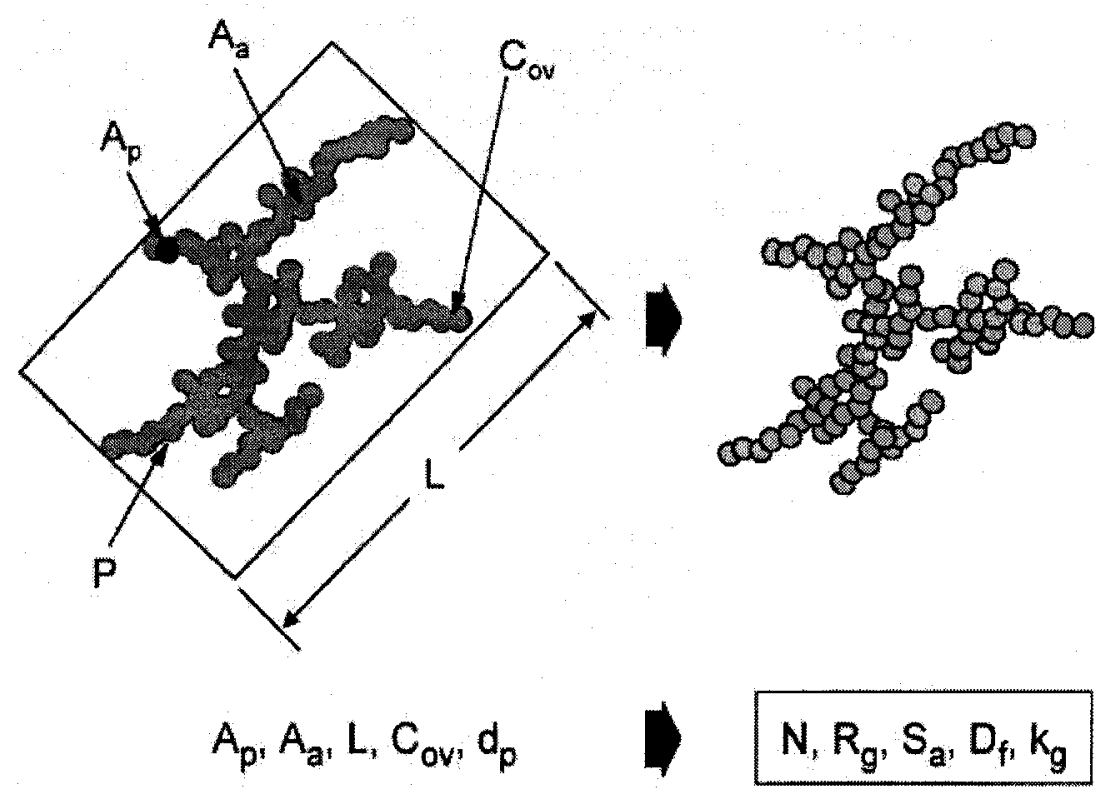

Figure 3.13: Experimental measurements and determinable parameters. Note that the overlap coefficient $C_{o v}$ and the surface area $S_{a}$ were not used in the present analysis.

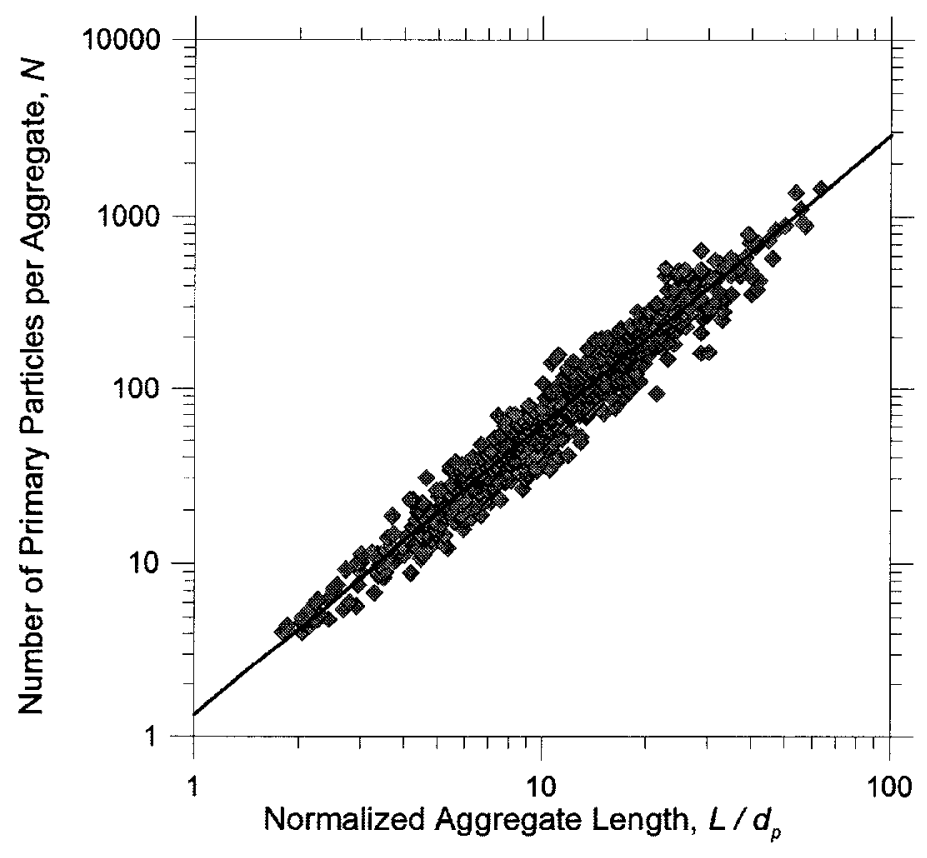

Figure 3.14: Number of primary particles versus aggregate length (condition A1) 
and size data were constrained to the range of $5<N<500$, where the histogram frequencies were of statistical significance (with the exception of conditions E2 and E3, which required modification to $20<N<800$ to converge with meaningful results). The fits were done using the Mathcad 14 LeastSquaresFit function, part of the Mathcad Data Analysis Extension Pack, which returns confidence limits on the resulting fits to the data. Mathcad analysis files are available in Appendix B. Figure 3.15 shows fits with $95 \%$ confidence limits for both distribution types, plotted on a $\log -\log$ scale. Unfortunately, in some cases the confidence limits were quite wide for both distributions, introducing a significant amount of uncertainty, and it was unclear at this point which one provided the best fit to the data.

These fits provided $x$ and $u x$ in the probability distribution $P(N ; x, u x)$, thus completing the morphological description of the soot. This information could then be used to calculate $\rho_{s a, \lambda}$, as discussed in the following section.

\subsection{Numerical Calculation of $\rho_{s a}$}

The numerical implementation of the scattering theory discussed in Chapter 2 is derived from the Mathcad analysis routines developed by Dr. Snelling of the combustion group at the National Research Council Institute for Chemical Process and Environmental Technology (NRC-ICPET). The relevant Mathcad analysis files are included in Appendix B.

As discussed in Section 2.4.4, $\rho_{s a, \lambda}$ is a function of the soot morphology, the wavelength of light, and the ratio of scattering to absorption refractive index functions, $F(m)_{\lambda} / E(m)_{\lambda}$. The latter value is the only remaining unknown, but fortunately this ratio has been reported in the literature. In particular, Krishnan et al. [2000] report a correlation for the wavelength range of $351-633 \mathrm{~nm}$, which they found through 


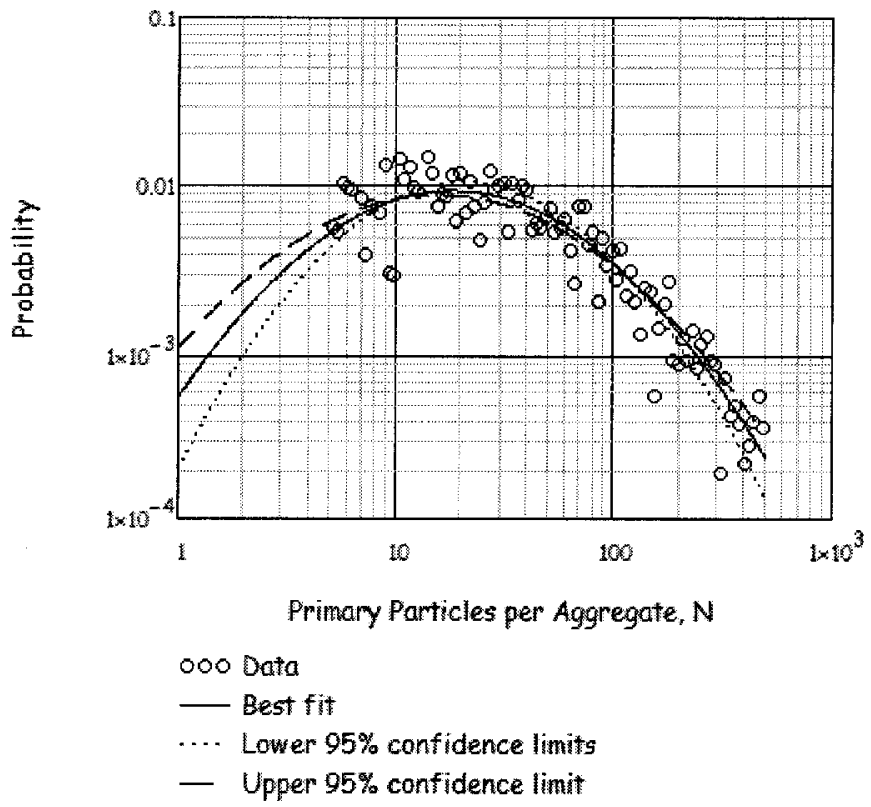

(a)

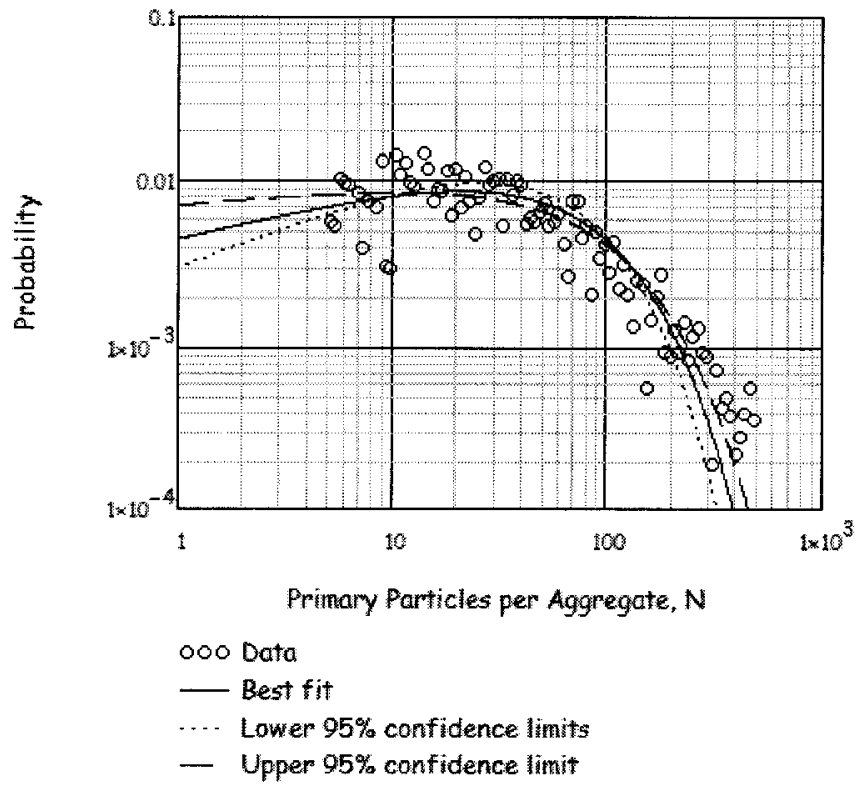

(b)

Figure 3.15: Fits of (a) log-normal and (b) self-preserving distributions to the aggregate size data 
their combined scattering/extinction measurements, gravimetric analysis, TEM, and reported values of $k_{g}$ from the previous work of Köylü et al. [1995a]. They validated their findings by correcting the previous extinction measurements of Köylü and Faeth [1996] with their current $\rho_{s a}$ values to find $E(m)$, and then applying their $F(m)_{\lambda} / E(m)_{\lambda}$ correlation to determine $F(m)$. They found that their experimentally-determined $E(m)$ and $F(m)$ and those extended from the work of Köylü and Faeth [1996] agreed quite well for wavelengths above $400 \mathrm{~nm}$.

The correlation of Krishnan et al. [2000] was curve-fitted to a power-law relationship in order to extrapolate to the higher wavelengths considered here. The expression used in the calculations is as follows:

$$
\left(\frac{F(m)}{E(m)}\right)_{\lambda}=0.9118 \cdot \ln (\lambda)-4.7198
$$

The use of this curve-fit extrapolation likely introduces further uncertainty in this calculation; however, no other reasonable alternative was found. Of those studies listed in Section 1.3 with sufficient data to determine a correlation, most have been questioned in the past for one reason or another [e.g. Köylü and Faeth, 1996, Krishnan et al., 2000]. For example, the ex-situ measurements made by Dalzell and Sarofim [1969] and Stagg and Charalampopoulos [1993] were performed on compressed soot pellets, which have been criticized for potentially altering the physical properties of soot. In addition, the earlier in-situ methods [e.g. Lee and Tien, 1981, Chang and Charalampopoulos, 1990] typically neglected characterization of soot morphology [Köylü and Faeth, 1996] and employed Rayleigh or Mie scattering theories, which have been found to be inappropriate for soot, as discussed in Chapter 2. Thus, the extrapolated correlation of Krishnan et al. [2000] was deemed the most appropriate choice for this work. 
The inputs to the Mathcad sheet are the morphological parameters discussed in the previous sections: primary particle diameter, fractal dimension and prefactor, and aggregate size distribution parameters for both log-normal and self-preserving distributions. The scatter-to-absorption ratio was calculated by numerical integration of Equation (2.31) at 25-nm increments to correspond to the extinction measurements, which are discussed in the following section. The transition between the explicit form of the structure factor given by Equation (2.16) to the power law form with coefficient $C$ given by Equation (2.18) occurred at $\frac{\left(q R_{g}\right)^{2}}{D_{f}}=42$, an empirical value found to work well in previous work at the NRC [Snelling, 2008]. Uncertainty was propagated by repeating the calculation using the lower and upper confidence limits of all the input parameters to observe the effect on the results. The outputs of the Mathcad sheet were tables of calculated $\rho_{s a, \lambda}$ values with fractional uncertainty limits (95\% confidence) for each wavelength bin.

\subsection{Spectral Line of Sight Attenuation}

Line of sight attenuation is an extinction measurement where the incident $\left(I_{\lambda 0}\right)$ and transmitted light $\left(I_{\lambda}\right)$ are ratioed to obtain the transmissivity, $\tau$, given by Equation (1.14). In this research, an imaging spectrometer (American Holographic, cat. no. SP00298-00) and CCD (Princeton Instruments, 7386-0001) were used to capture spectrally-resolved extinction coefficients over the range of visible and near-infrared wavelengths of $450-950 \mathrm{~nm}$. Since the spectral intensity of the lamp decreased toward the upper wavelengths, sensitivity of the diagnostic at higher wavelengths was reduced. To mitigate this effect, it was necessary to break measurements into two data sets, the first to capture the range of $450-750 \mathrm{~nm}$, and the second to capture $675-950 \mathrm{~nm}$. The higher range was filtered to eliminate light below $650 \mathrm{~nm}$, which 
increased the sensitivity in that range while eliminating so-called second-order effects. Second-order effects occur when radiation at a wavelength $\lambda$ is imaged at the same location as radiation at $2 \lambda$, inducing optical contamination that is proportional to the intensity ratio between the two wavelengths [Thomson et al., 2008a]. Without this filtering approach, this mechanism contaminated the range of $700-950 \mathrm{~nm}$ due to the relatively strong incident radiation at $350-475 \mathrm{~nm}$.

The system is shown schematically in Figure 3.16, and as a photograph in Figure 3.17. The light source was a simple consumer-grade $12-\mathrm{V}, 50-\mathrm{W}$ DC halogen bulb (Noma), that was fed into an integrating sphere, which produced uniform, diffuse light at its outlet. The halogen bulb was powered by a bench top power supply to ensure constant input power. The use of diffuse light to make low-noise transmissivity measurements is discussed in detail in [Thomson et al., 2008b]. A typical low-wavelength image taken by the system can be seen in Figure 3.18, where the horizontal axis is spatial, representing a horizontal line through the exhaust plume, and the vertical axis is spectral (higher wavelengths toward the top). Three images were collected for each measurement, which were referred to as plume, lamp, and dark. The dark image was taken with the light source off and the plume blocked (the exhaust gases were redirected through the water drain, and the burner exit was sealed with a layer of waxed paper), to measure ambient light and so-called dark counts on the CCD. The lamp image was taken with the lamp on and the plume blocked, to measure the incident light. Finally, the plume image was taken with the lamp on and the plume flowing, to measure the transmitted light. The transmissivity of the plume was then found according to the relation

$$
\tau_{\lambda}=\frac{p l u m e-d a r k}{l a m p-d a r k}=\frac{I_{\lambda}}{I_{\lambda 0}}
$$




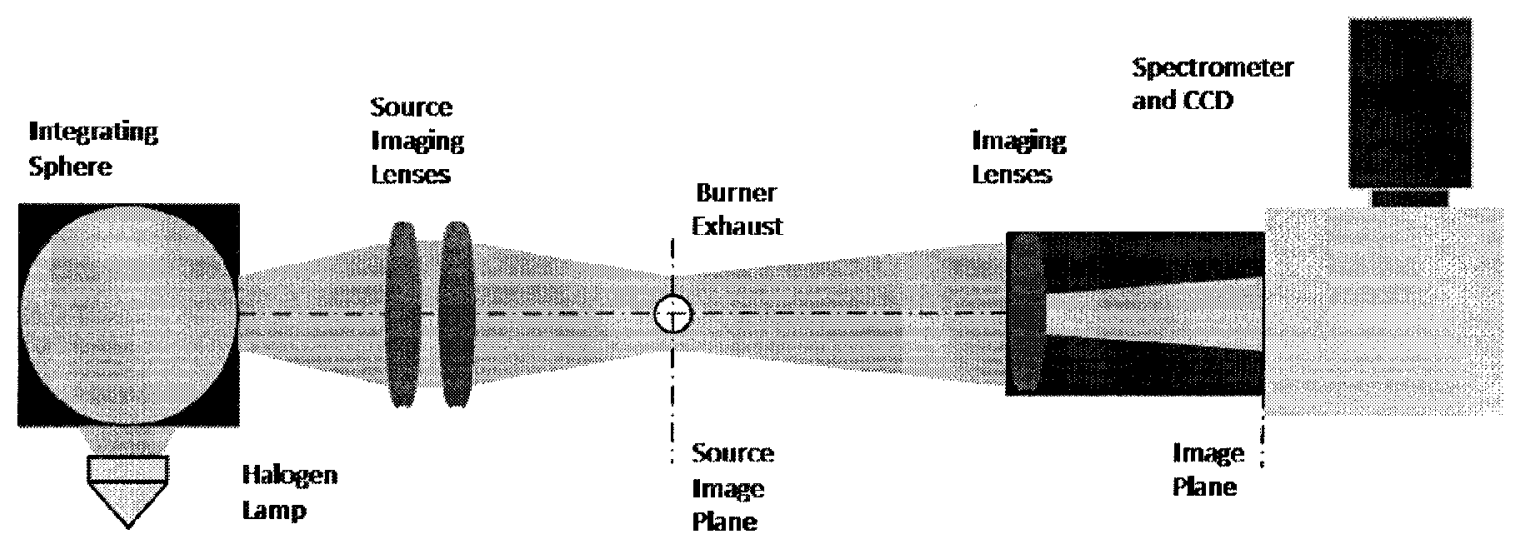

Figure 3.16: Schematic of Spec-LOSA experiment

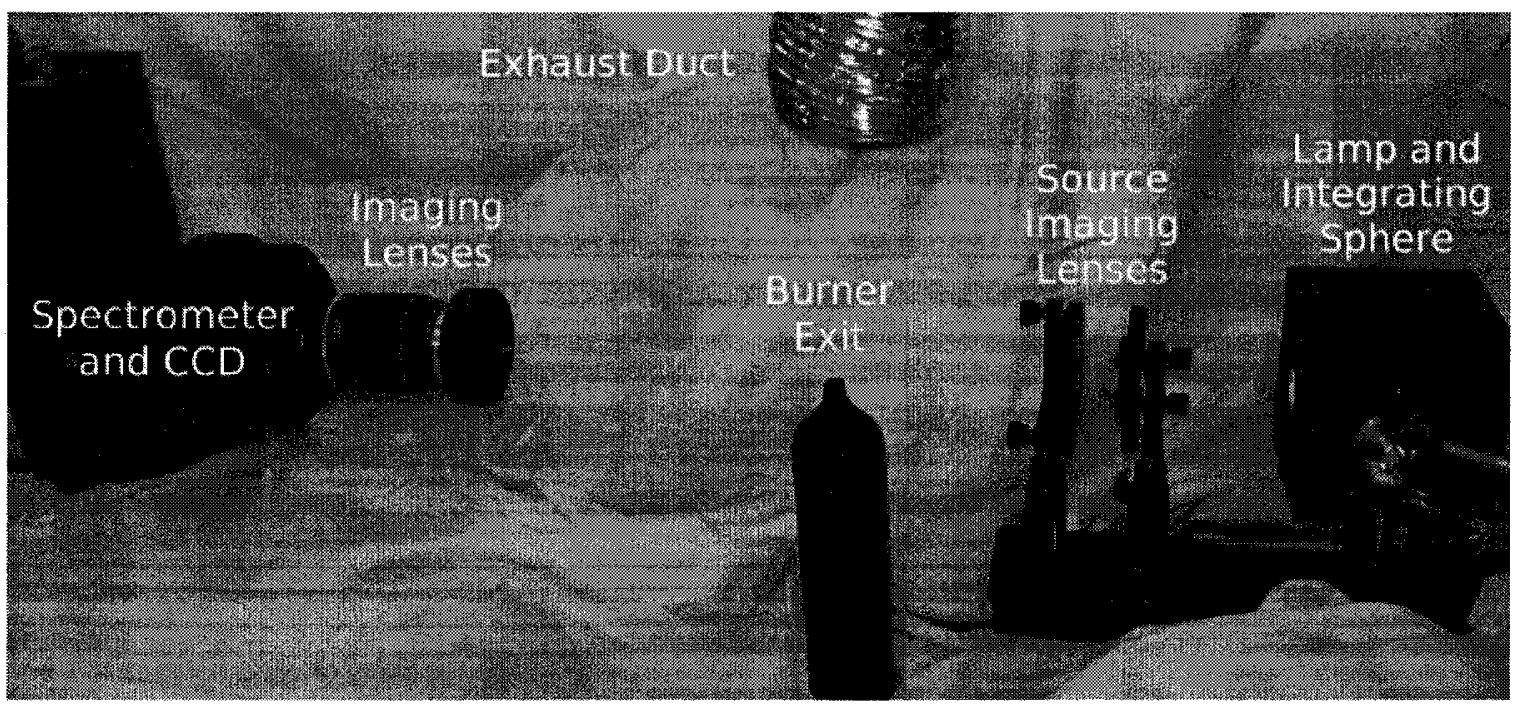

Figure 3.17: Photograph of LOSA setup 


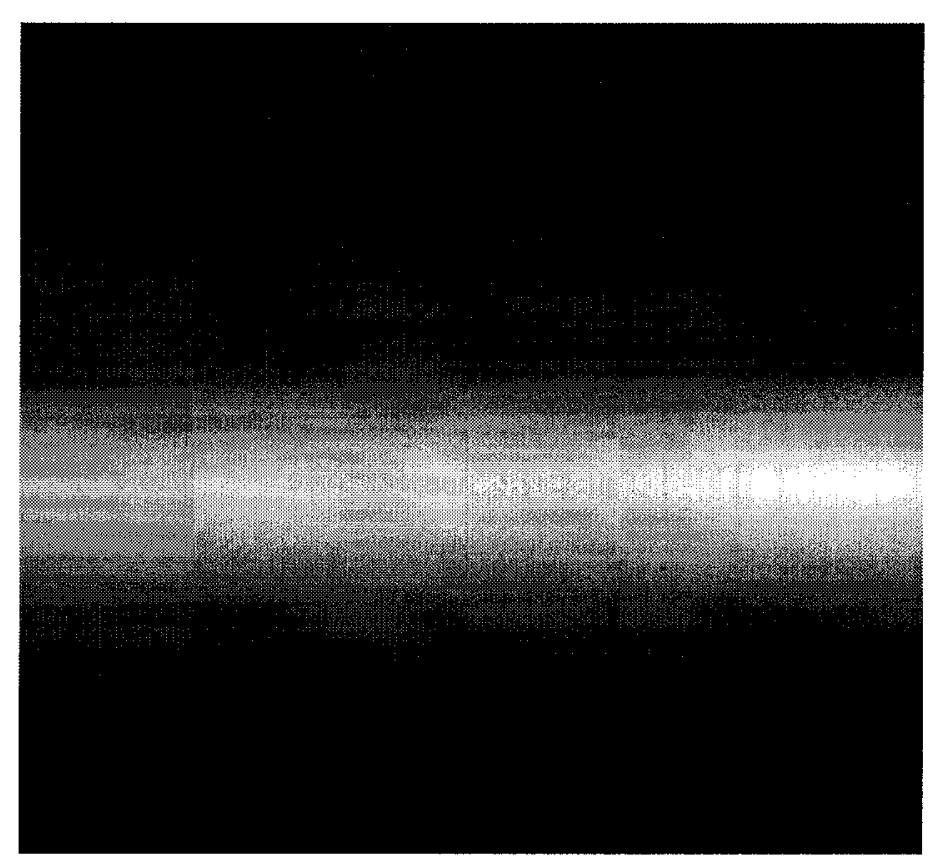

Figure 3.18: Typical unfiltered (low-wavelength set) Spec-LOSA image

\subsubsection{Spec-LOSA Analysis}

Raw image data from the CCD was binned in both vertical wavelength increments of $25 \mathrm{~nm}$ and to a horizontal spatial resolution of approximately $0.1 \mathrm{~mm}$ (3 pixels). The transmissivity of each bin was then found by Equation (3.6), and the resulting data were normalized so that the transmissivity of the unattenuated portion of the image was equal to unity, as seen in Figure 3.19. As expected, the transmissivity was proportional to the length of the optical path through the attenuating medium, which in this case was the chord length through the circular plume. If the diameter of the circular cross section was well-known, this data could have been used immediately by dividing the total transmissivity by the chord length as in Equation (1.15). However, since the plume was unconfined at the location of measurement, some dispersion and entrainment of air was bound to occur, which would result in a non-uniform extinction coefficient near the edges of the plume. This issue was addressed through the use of 


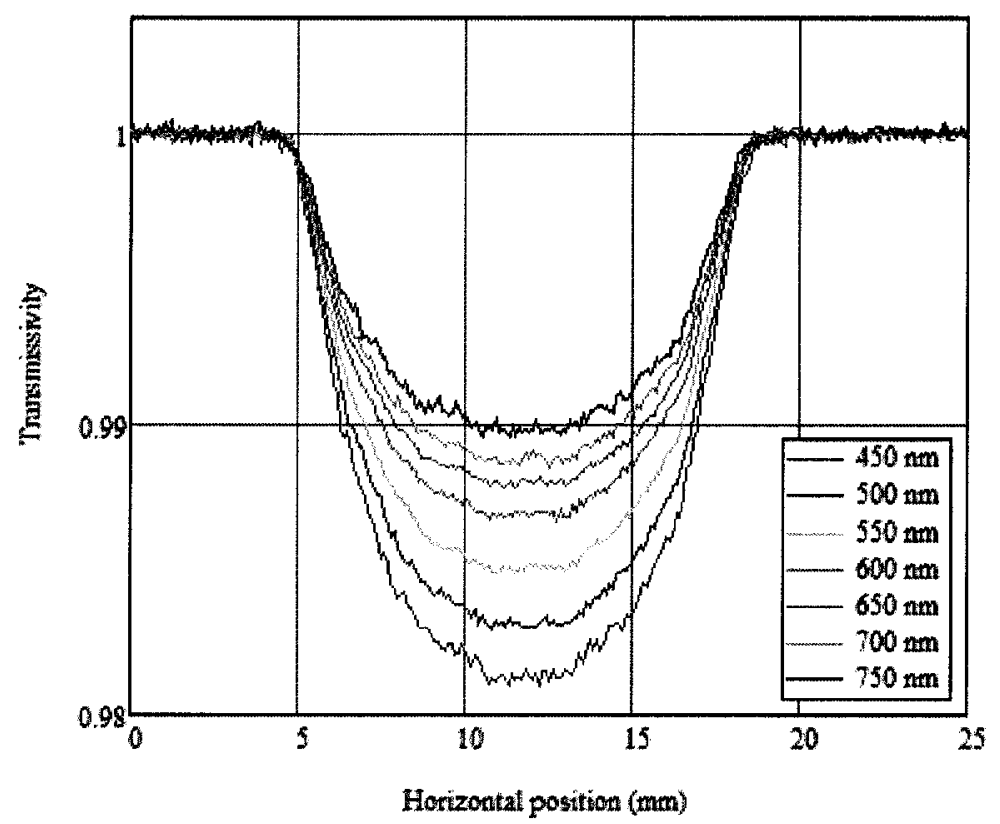

Figure 3.19: Normalized transmissivity profile across plume (condition D3)

1-D tomography to invert the data and obtain a radial transmissivity profile.

A three-point Abel inversion method was employed to deconvolve the projected transmissivity data into a radial profile. This method has in the past been successfully used in flame emission measurements [Ayranci et al., 2007] and 2-D LOSA measurements [Snelling et al., 1999], and was the method recommended by Dasch [1992] for its low noise, speed, and robustness in a comparison of Abel inversion, onion-peeling, and filtered backprojection methods. Details of this method can be found in Dasch [1992]. Since inversion algorithms amplify noise, the data were first smoothed using the Mathcad loess function for least-squares fitting, the result of which can be seen in Figure 3.20.

The uniformity in the radial transmissivity seen in Figure 3.21 confirms the assumption that the extinction coefficient is spatially uniform in the plume. To avoid entrainment effects, the extinction coefficient was determined from the data in the 


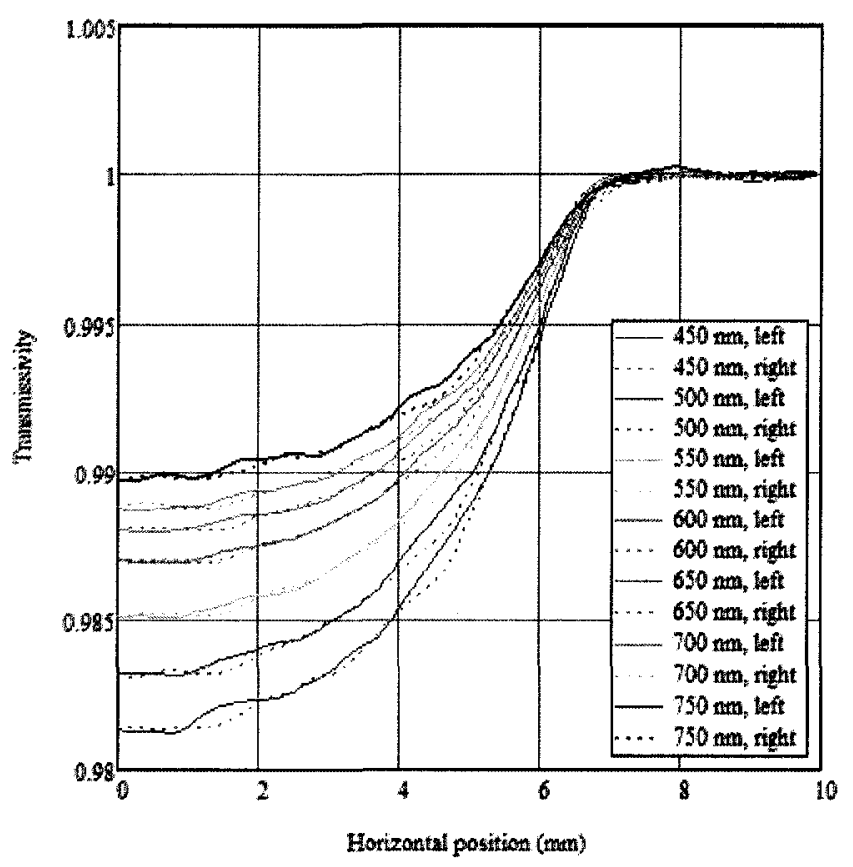

Figure 3.20: Smoothed transmissivity profile from centreline (condition D3)

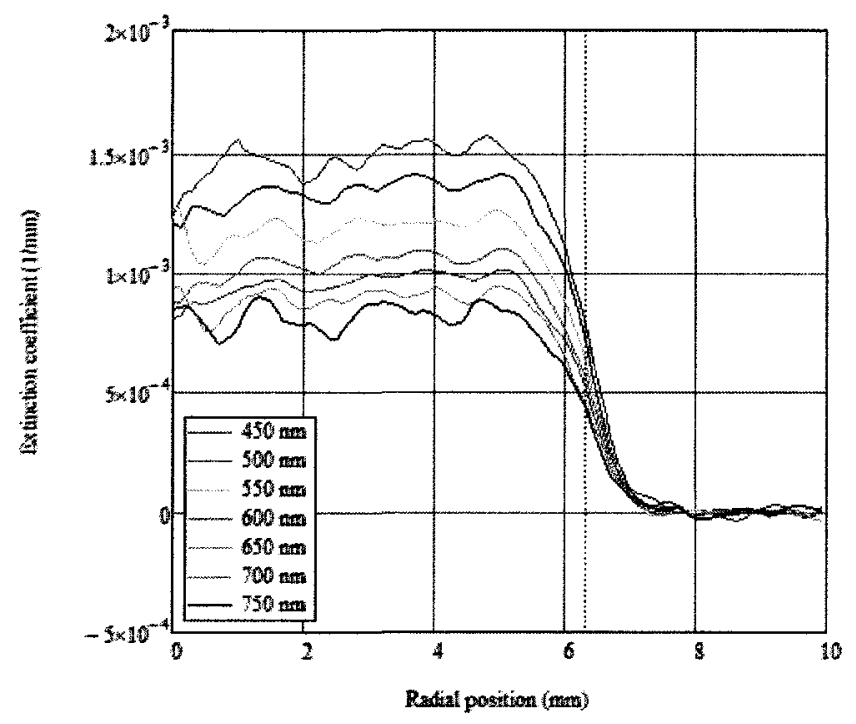

Figure 3.21: Radial profile found by tomographic inversion (condition D3). The vertical dashed line indicates the edge of the burner exit nozzle. 
central region with radii of 0 to $5 \mathrm{~mm}$ only.

\subsection{Laser-Induced Incandescence (LII)}

The LII apparatus used for the present research was nearly identical to that described by Snelling et al. [2005]. A schematic of that system can be seen in Figure 3.22. The key difference between that system and the one used here is that in the present system, the detection optics were fiber coupled to the detection package rather than directly coupled; the systems are equivalent if the time taken for the signal to travel the length of the fiber is taken into account. The light source was a frequency-doubled, pulsed Nd:YAG laser with output at $532 \mathrm{~nm}$ at $20 \mathrm{~Hz}$. Output energy was controlled by a half-wave plate and thin-film polarizer. The laser beam was shaped into a rectangle by means of a vertical slit and a spherical lens to obtain a quasi-uniform intensity profile at the probe volume. LII radiation was transmitted by a fiber to the receiving optics, where it was split into wavelength components by beam splitters, passed through narrow-band interference filters with centre wavelengths of $444.919 \mathrm{~nm}$ and $782.756 \mathrm{~nm}$, and directed onto photomultiplier tube detectors (PMTs). The signal from the PMTs are digitized by a digital oscilloscope and recorded by a LabVIEW virtual instrument. The reader is referred to Snelling et al. [2005] for a more detailed description of the experimental apparatus and the theory behind it.

Data were obtained for the twelve burner conditions, each over a range of laser energies $\left(2-6 \frac{\mathrm{mJ}}{\text { pulse }}\right.$, or fluences of $\left.0.55-1.65 \frac{\mathrm{mJ}}{\mathrm{mm}^{2}}\right)$. Each data point was the averaged result of 300 individual measurements (three runs of 100 measurements each), collected over the course of several days.

Soot volume fraction was determined via Equation (1.10), given the temperature found by Equation (1.13). Both parameters depended on values of $E(m)_{\lambda}$ for the 


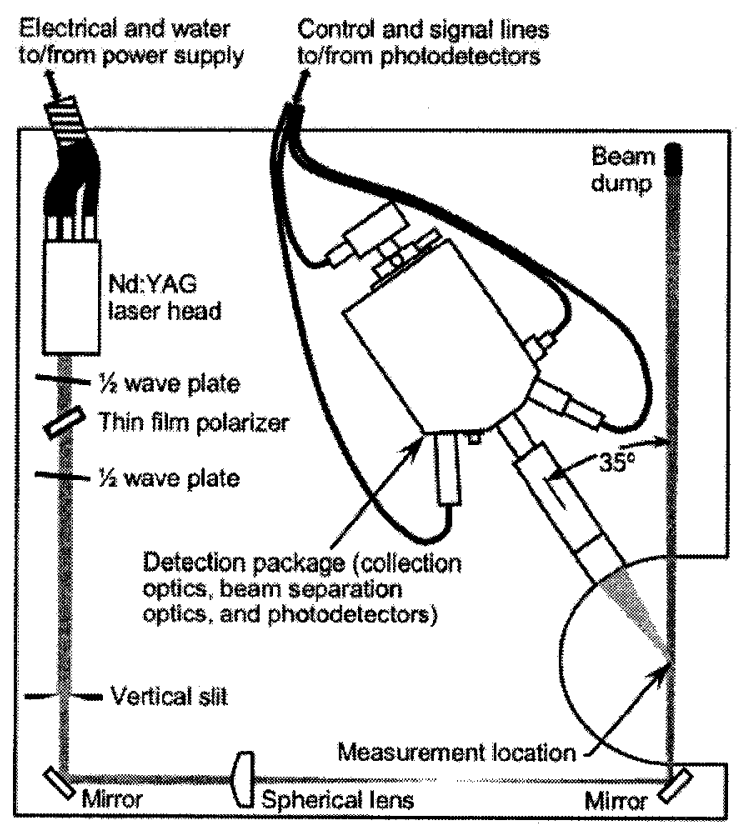

Figure 3.22: Schematic of LII system [Snelling et al., 2005]

two centre wavelengths given above. An initial analysis was done using the values of Snelling et al. [2004] (i.e. a wavelength-independent value of 0.4), and the data were re-analyzed using the $E(m)_{\lambda}$ results of the present study for comparison. The results are presented in Section 4.5.

\subsection{Uncertainty Analysis}

Experimental results are somewhat meaningless without an idea of the accuracy of the measurements. The uncertainties associated with the values obtained were estimated based on the method outlined in [ASME, 1985]. The total uncertainty consists of two distinct parts, the bias error and the precision error. Bias error occurs due to the inaccuracy of the measuring equipment, whereas the precision error occurs due to random scatter of the data. These two types of uncertainty are evaluated individually and then combined to find an estimate of the total uncertainty. 


\subsubsection{Bias Error}

The bias error consists of the inherent measurement uncertainties of the instruments doing the measuring. Since no measurement system can get perfectly accurate values, it is impossible to precisely determine the so-called bias limit of an instrument - it must instead be estimated. In the propagation of bias error, it is assumed that there are roughly as many positive errors as negative ones, and the net result can be estimated by combining the individual bias limits in quadrature. However, this assumption becomes less valid when the number of bias limits being combined is small, since they can actually add constructively to produce a larger error. Since in this research there are myriad sources of uncertainty, it was deemed quite reasonable to sum individual bias limits in quadrature.

In general terms, the bias error of some function $f\left(x_{1}, x_{2}, \ldots x_{n}\right)$ propagates forward according to Equation (3.7), where $U_{f}$ is the propagated uncertainty on $f$, and $U_{1}, U_{2}, \ldots, U_{n}$ are the bias limits of $x_{1}, x_{2}, \ldots, x_{n}$, respectively.

$$
U_{f}=\sqrt{U_{1}^{2}+U_{2}^{2}+\cdots+U_{n}^{2}}
$$

These bias limits are in turn calculated using the derivative method, as follows:

$$
U_{1}=\frac{\partial f\left(x_{1}, x_{2}, \ldots, x_{n}\right)}{\partial x_{1}} U_{x_{1}}
$$

where $U_{x_{1}}$ is the bias limit of the variable $x_{1}$.

For simple cases where the formula for $f$ consists only of multiplication or division

of its variables (with no exponents), Equation (3.7) can be simplified into fractional 
or percentage uncertainties, as follows in Equation (3.9):

$$
\frac{U_{f}}{f}=\sqrt{\left(\frac{U_{x_{1}}}{x_{1}}\right)^{2}+\left(\frac{U_{x_{2}}}{x_{2}}\right)^{2}+\cdots+\left(\frac{U_{x_{n}}}{x_{n}}\right)^{2}}
$$

\subsubsection{Precision Error}

The precision error of a measurement describes the scatter in the results of repeated measurement of the same data point. The average of all measurements is reported, and the spread in data is described by the precision error, typically in the form of 95\% confidence limits. The confidence limits are determined according to Student's $t$-test, which accounts for the number of measurements taken and the fact that the standard deviation, $\sigma$, of the sample population may not be the same as that for the overall population. The $t$ distribution has only one parameter, called the number of degrees of freedom $(d f)$, which is simply $n-1$ for cases such as this. Note that the $t$ distribution approaches a normal distribution as $d f \rightarrow \infty$ [Devore, 2004].

The $100(1-\alpha) \%$ confidence limits associated with a mean value, $\bar{x}$, is determined by the following formula:

$$
\bar{x} \pm t_{\frac{\alpha}{2}, n-1} \frac{\sigma}{\sqrt{n}}
$$

where the term $t_{\frac{\alpha}{2}, n-1} \frac{\sigma}{\sqrt{n}}$ represents the precision error. The term $\frac{\sigma}{\sqrt{N}}$ is called the standard error of the mean, or just the standard error.

The total uncertainty on a measurement due to both bias and precision errors is found by combining these two contributions in quadrature, as in

$$
\frac{U_{x, \text { total }}}{x}=\sqrt{\left(\frac{U_{x, b i a s}}{x}\right)^{2}+\left(\frac{U_{x, \text { precision }}}{x}\right)^{2}}
$$

Results of the uncertainty analysis will be presented alongside the measurement re- 
sults in the following Chapter. 


\section{Chapter 4}

\section{Results}

\subsection{Gravimetric Analysis}

Gravimetric analysis was performed for the twelve conditions described in the previous Chapter in Table 3.1. Filter masses were on the order of $50 \mathrm{mg}$, and the masses of the collected soot samples were two orders of magnitude less, typically $150-300 \mu \mathrm{g}$. The results of the analysis are tabulated in Table 4.1, which shows the measured mean soot volume fraction, along with the $95 \%$ confidence interval found by Equation (3.10).

Table 4.1: Gravimetric soot volume fractions, in parts per billion (ppb)

\begin{tabular}{|c|c|c|c|c|c|c|c|c|c|c|c|c|}
\hline$[\mathrm{ppb}]$ & $\mathrm{A} 1$ & B1 & B2 & C1 & C2 & C3 & D1 & D2 & D3 & E1 & E2 & E3 \\
\hline \hline$f_{v}$ & 64.7 & 106 & 103 & 61.5 & 63.4 & 54.9 & 72.2 & 75.5 & 76.5 & 87.1 & 87.7 & 91.8 \\
\hline $95 \%$ CI & 3.2 & 5.1 & 4.8 & 2.9 & 3.0 & 2.6 & 3.4 & 3.5 & 3.7 & 4.1 & 4.3 & 4.2 \\
\hline
\end{tabular}

\section{Uncertainty}

The soot volume fraction determined by gravimetric analysis is dependent on the mass of the collected soot, the volume of gas that was filtered, the ambient and gas temperatures, and the mass density of soot. The remaining uncertainty on the mass 
of soot is captured in the repeatability of the balance, which was found by taking repeated measurements of a single sample, both sequentially (sooty and non-sooty samples, 41 measurements each with standard deviations of $0.001 \mathrm{mg}$ ) and over the course of about eight weeks (sooty filter, 31 samples with a standard deviation of $0.002 \mathrm{mg}$ ). The variability was found to be an order of magnitude greater than the quoted accuracy of the balance, which is $0.0001 \mathrm{mg}$. Since the mass of soot determined is the result of two separate mass measurements, the uncertainty was taken to be $\sqrt{2} \cdot 0.002 \approx 0.003 \mathrm{mg}$.

The gas volume flow rate was monitored using a Brooks thermal mass flow controller (MFC), hardware timed to acquire data at $10 \mathrm{~Hz}$. The total volume was found by multiplying the flow rate by the time interval at each time step and summing. The uncertainty associated with this measurement corresponded to the volume measured in two time steps, combined in quadrature with the quoted uncertainty of the MFC ( $\pm 0.2 \%$ of full scale $\pm 0.7 \%$ of reading). Ambient temperature and exhaust gas temperature were measured with K-type thermocouples (Omega) with reading uncertainties of $2.2 \mathrm{~K}$. However, the ambient measurement was made in the room rather than in the sampled stream, and it was assumed that the gas had fully cooled to ambient temperature at the point of measurement by the MFC. It was noticed while cleaning the thermocouple probe that the exhaust temperature measurement was slightly sensitive to soot buildup (though this is less of an issue in the present case of steady-state measurements, where the wall and gas temperatures are similar). To account for these additional sources of error, the thermocouple uncertainties were inflated to $5 \mathrm{~K}$ each. The uncertainty on the mass density of soot corresponds to the spread of the values reported in the literature (95\% confidence interval), which are listed in Table 4.2 .

For the example case of a single gravimetric sample of condition C3, the bias limit 
Table 4.2: Values of the mass density of soot from literature

\begin{tabular}{|c|c|c|}
\hline Source & Fuel & $\rho_{s}\left[\frac{\mathrm{g}}{\mathrm{cm}^{3}}\right]$ \\
\hline \hline Le Chatelier (1926)* & Acetylene black & 1.95 \\
\hline Rossman and Smith (1943)* $^{*}$ & Acetylene black & 1.84 \\
\hline Janzen (1980)* & Carbon black & 1.84 \\
\hline Roessler and Faxvog (1980)* & Acetylene flame & 2.05 \\
\hline Nishida and Mukohava (1983)* & Propane flame & 1.95 \\
\hline Nelson (1989)* & Acetylene flame & 1.90 \\
\hline Choi et al. [1994] & Acetylene/air (premixed) & 1.84 \\
\hline Choi et al. [1995] & Acetylene/air (premixed) & 1.74 \\
\hline Wu et al. [1997] & Various & 1.89 \\
\hline & Mean: & 1.89 \\
\cline { 2 - 3 } & $95 \%$ CI: & 0.07 \\
\cline { 2 - 3 }
\end{tabular}

* Values from data summary given by Dobbins et al. [1994]

was then (where $U_{x}$ is the uncertainty in $x$ )

$$
\begin{aligned}
\frac{U_{f_{v}}}{f} & =\sqrt{\left(\frac{U_{m_{s}}}{m_{s}}\right)^{2}+\left(\frac{U_{V}}{V}\right)^{2}+\left(\frac{U_{T_{a}}}{T_{a}}\right)^{2}+\left(\frac{U_{T_{s}}}{T_{s}}\right)^{2}+\left(\frac{U_{\rho_{s}}}{\rho_{s}}\right)^{2}} \\
& =\sqrt{\left(\frac{0.003 \mathrm{mg}}{0.172 \mathrm{mg}}\right)^{2}+\left(\frac{0.015 \mathrm{~L}}{1.497 \mathrm{~L}}\right)^{2}+\left(\frac{5 \mathrm{~K}}{298 \mathrm{~K}}\right)^{2}+\left(\frac{5 \mathrm{~K}}{331 \mathrm{~K}}\right)^{2}+\left(\frac{70 \mathrm{~kg} / \mathrm{m}^{3}}{1890 \mathrm{~kg} / \mathrm{m}^{3}}\right)^{2}} \\
& =4.8 \%
\end{aligned}
$$

The average bias error for all 34 samples from condition C3 was found to be $4.6 \%$. The average soot volume fraction was 54.9 parts per billion (ppb) with a standard deviation of $2.0 \mathrm{ppb}$ or $3.7 \%$. The precision error for $95 \%$ confidence was, from Equation (3.10),

$$
t_{2.5,33} \frac{\sigma_{f_{v}}}{\sqrt{n}}=2.035 \frac{3.7 \%}{\sqrt{34}}=1.3 \%
$$

Thus, the total uncertainty on the gravimetric soot volume fraction for condition C3 was then:

$$
\sqrt{(4.6)^{2}+(1.3)^{2}}=4.8 \%=2.6 \mathrm{ppb}
$$




\subsection{Spectral Line of Sight Attenuation}

The spectral line-of-sight attenuation diagnostic discussed in Section 3.5 was also applied to the twelve burner conditions listed in Table 3.1, with and without the coloured filter to block out the lower wavelengths. As mentioned in the previous chapter, two data sets were taken for each burner condition; four wavelength bins (675, 700, 725, and $750 \mathrm{~nm}$ ) were common to both sets. In all conditions, error bars from these common wavelengths overlap, indicating that the slight differences that do exist are likely a result of measurement noise. These data could arguably be averaged to obtain one continuous function of $K_{e x t, \lambda}$ versus wavelength; however, they are kept distinct to preserve data integrity and to acknowledge that the data do come from two distinct measurements. These two wavelength ranges for the same condition are simply concatenated, including overlap, to make up one single, full set; unless otherwise indicated, no further distinction will be made between data from the upper and lower wavelength sets.

Figure 4.1 shows the full data set for condition A1 (chosen arbitrarily); the trends apparent in this condition are representative of all conditions. Of particular note is the monotonic decrease of $K_{\text {ext, } \lambda}$ with increasing wavelength. Figure 4.2 illustrates the effect of dilution rate on the measured extinction; recall from Table 3.1 that the "C" series has a dilution rate of 50 SLPM (or dilution ratio of 3.1), the "D" series a dilution rate of 40 SLPM (2.5) and the "E" series a dilution rate of 30 SLPM (1.9). The differences seen in the extinction coefficient between dilution rates are predictable, as this parameter scales the soot concentration.

Figure 4.3 displays the full range of extinction measurements made. Note that the ordinate axis is stretched and truncated in an effort to distinguish data sets. An interesting feature is that all room-temperature data sets (3-series and A1) are distin- 


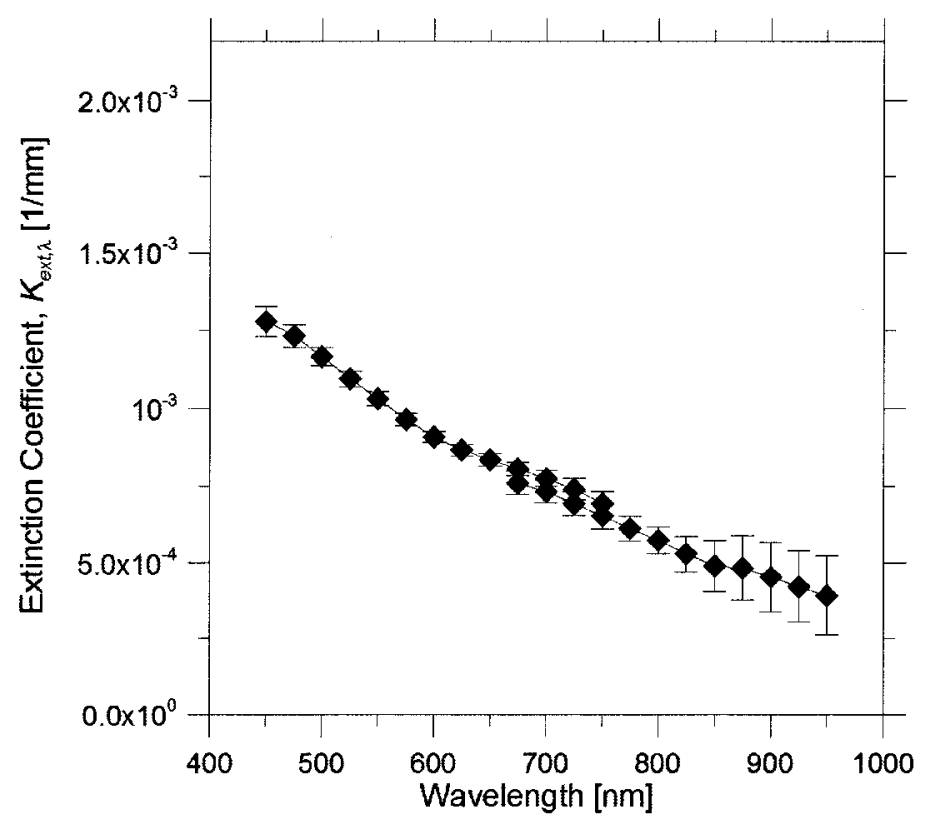

Figure 4.1: Specular extinction coefficients for condition A1

guished from their higher-temperature counterparts (1- and 2-series). Although the differences are minor, this does reinforce the conceptual argument that temperature history does play some role in the resulting optical properties of soot.

\section{Uncertainty}

Each wavelength-dependent extinction coefficient is an average value determined from 30 individual measurements. The precision uncertainty was determined by Equation (3.10), where the $t$-value for 29 degrees of freedom at a $95 \%$ confidence limit is 2.045 . The lamp intensity was strongest at the middle wavelengths, increasing the signalto-noise ratio, which was apparent in the uncertainty data. The reported extinction coefficient was found by Equation (1.15) and was spatially uniform, so the choice of $L$ was somewhat arbitrary. The bias limits could therefore be expressed as

$$
\frac{U_{K_{e x t, \lambda}}}{K_{e x t, \lambda}}=\left|\frac{U_{\ln \left(\tau_{\lambda}\right)}}{\ln \left(\tau_{\lambda}\right)}\right|
$$




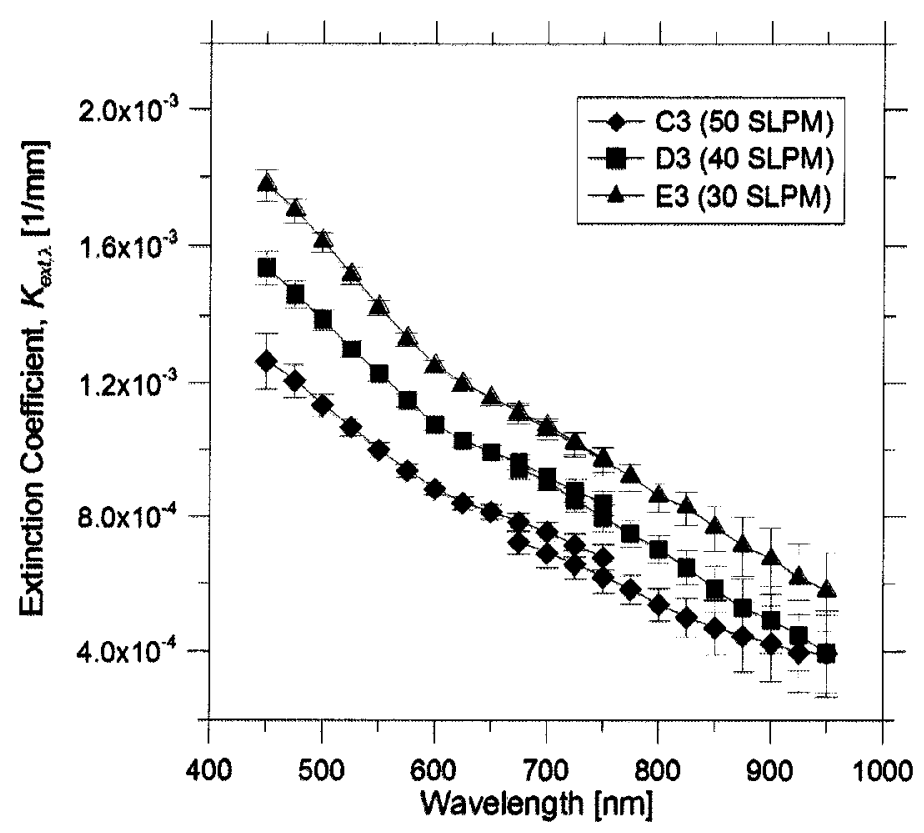

Figure 4.2: $K_{\text {ext, } \lambda}$ data comparing dilution rates, with the C-series being the most diluted and $E$ the least

$U_{\ln \left(\tau_{\lambda}\right)}$ was estimated by examining the symmetry about a vertical axis (perpendicular to the optical axis) in the normalized transmissivity data. Recall from the previous Chapter that LOSA measurements involve taking the ratio of intensities at corresponding locations of the 'plume' and 'lamp' images. The exhaust plume attenuates the lamp light in the central portion of the image; the edge regions of the image are unattenuated and therefore have unity transmissivity. Data were normalized using the unattenuated portion on the left-hand side of the images. Therefore, any deviations from unity transmissivity appearing in the unattenuated portion on the right-hand side were representative of a systematic (bias) error in the measurement of $\ln \left(\tau_{\lambda}\right)$. These small deviations can be seen, particularly at the upper wavelengths, in Figure 4.4 for condition C2, which had the lowest values of $K_{e x t, \lambda}$ and is thus the most prone to this type of measurement error. Values for $U_{\ln \left(\tau_{\lambda}\right)}$ were found by averaging the noise on the right side of the plume (x-positions 200-250 in the figure), and bias 


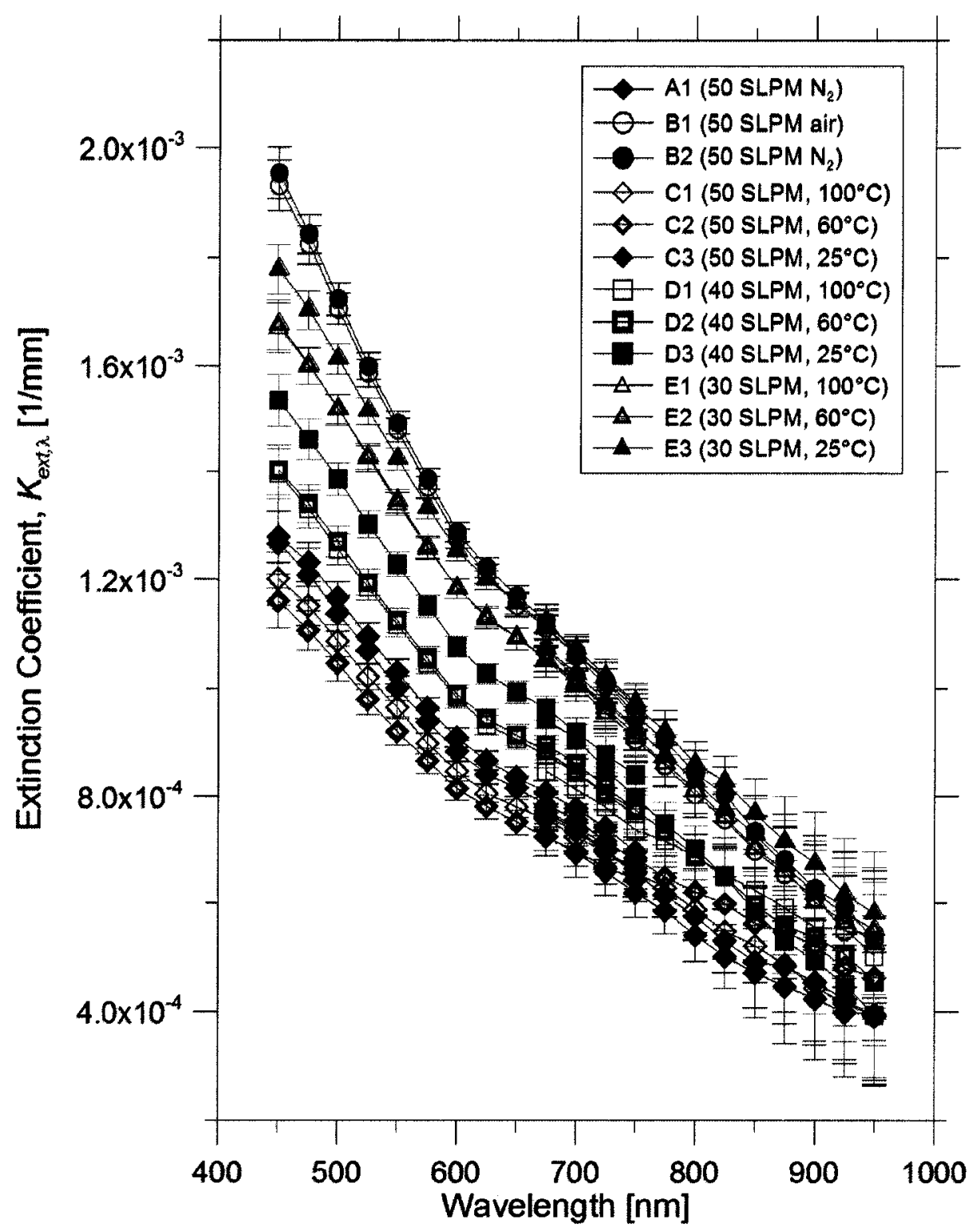

Figure 4.3: Comparison graph of all extinction data (refer to Table 3.1 on page 44 for further description of the burner conditions) 


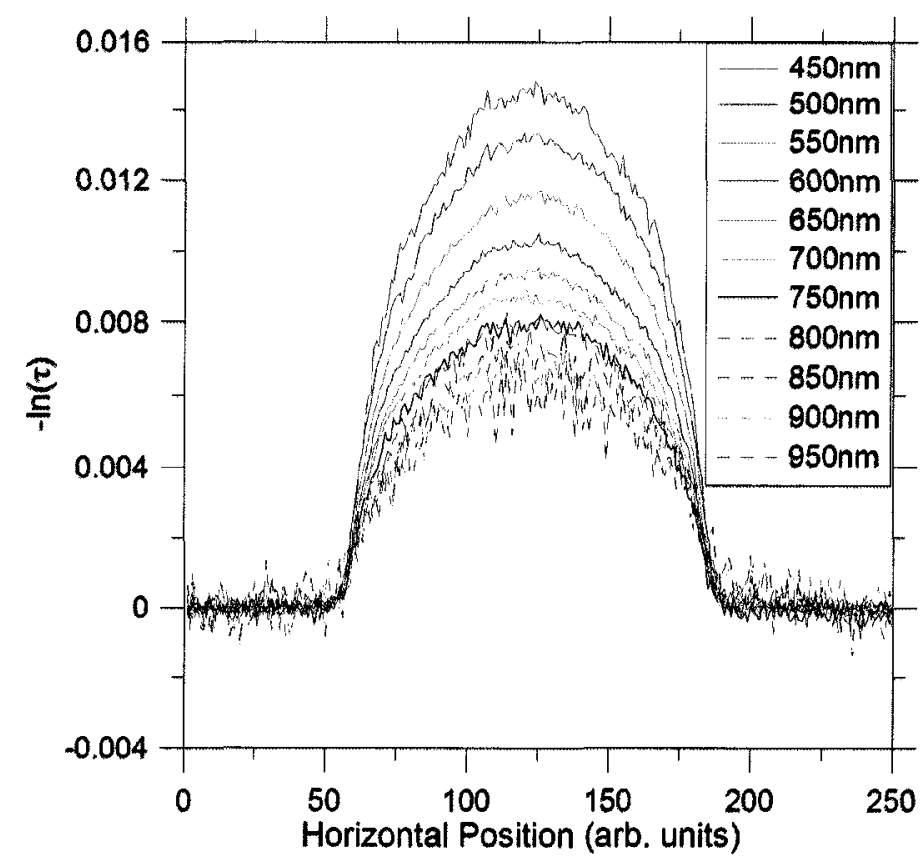

Figure 4.4: Normalized $\ln \left(\tau_{\lambda}\right)$ data for condition $\mathrm{C} 2$.

limits were found by dividing that by the corresponding peak values of $\ln \left(\tau_{\lambda}\right)$. Figure 4.5 shows the bias, precision, and combined uncertainties (summed in quadrature) for condition C2. The contribution of the bias uncertainty to the total uncertainty is negligible. Thus, only the precision uncertainty was calculated for the remaining conditions.

\subsection{Determination of Scatter to Absorption Ratio}

The scatter to absorption ratio was used to correct the extinction measurements for scatter in order to isolate the absorption component. The calculation of this parameter required soot morphology information, which was found through the exsitu diagnostics discussed in the following sections. 


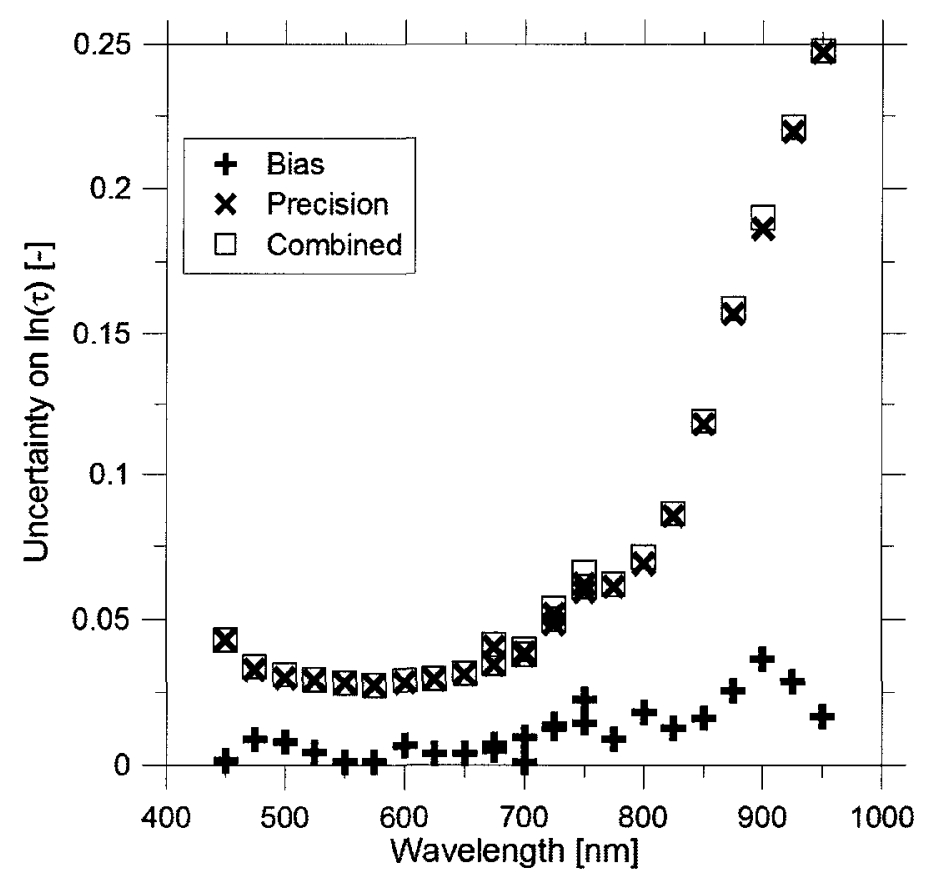

Figure 4.5: Comparison of bias, precision, and combined uncertainties in $\ln \left(\tau_{\lambda}\right)$ measurements for condition $\mathrm{C} 2$

\subsubsection{Primary Particle Diameter from TEM}

Primary particle diameter, $d_{p}$, was found by transmission electron microscopy for the twelve burner conditions given in Table 3.1. The results of this analysis are the mean primary particle diameter and the $95 \%$ confidence interval of the measurement, given in Table 4.3 .

Table 4.3: Primary particle diameters as measured by TEM

\begin{tabular}{|c|c|c|c|c|c|c|c|c|c|c|c|c|}
\hline$[\mathrm{nm}]$ & A1 & B1 & B2 & C1 & C2 & C3 & D1 & D2 & D3 & E1 & E2 & E3 \\
\hline \hline$d_{p}$ & 38.7 & 42.6 & 44.3 & 39.8 & 40.1 & 33.1 & 45.3 & 38.9 & 39.4 & 41.1 & 43.0 & 43.3 \\
\hline $95 \%$ CI & 2.2 & 2.7 & 2.5 & 2.4 & 2.4 & 2.2 & 2.9 & 2.5 & 2.4 & 2.6 & 2.4 & 2.5 \\
\hline
\end{tabular}

\section{Uncertainty}

The uncertainty calculation for the TEM results comprised bias and precision errors. Bias error was estimated based on the spatial resolution of the images. At a magnifi- 
cation of $18000 \mathrm{X}$, the spatial calibration was $0.777778 \mathrm{~mm} /$ pixel, and the accuracy of the location of particle edges was estimated at \pm 2 pixels. That error occurs twice, finding both edges of the particle, so the resulting bias error was $2 \sqrt{2} \cdot 0.777778 \approx 2.2 \mathrm{~nm}$. Precision error was found via Equation (3.10). Using the values from condition D2 as an example, a $95 \%$ confidence interval of $1.1 \mathrm{~nm}$ was obtained. Combining these contributions in quadrature resulted in a total uncertainty of $\sqrt{2.2^{2}+1.1^{2}}=2.5 \mathrm{~nm}$.

\subsubsection{Soot Aggregate Geometric Parameters from SEM}

The SEM image analysis described in Section 3.3.4 was performed on the twelve burner conditions to determine the fractal parameters and the aggregate size distributions.

\subsubsection{Fractal Parameters}

The fractal dimension and prefactor were found using the projected area method for aggregate characterization, discussed in Section 3.3.4. The results of this analysis are presented in Table 4.4 .

Table 4.4: Fractal properties as measured by SEM

\begin{tabular}{|c|c|c|c|c|c|c|c|c|c|c|c|c|}
\cline { 2 - 14 } \multicolumn{1}{c|}{} & A1 & B1 & B2 & C1 & C2 & C3 & D1 & D2 & D3 & E1 & E2 & E3 \\
\hline$D_{f}$ & 1.67 & 1.68 & 1.64 & 1.66 & 1.67 & 1.69 & 1.64 & 1.63 & 1.68 & 1.66 & 1.69 & 1.70 \\
\hline$k_{g}$ & 8.15 & 7.90 & 7.12 & 7.97 & 8.11 & 9.40 & 8.02 & 7.88 & 7.37 & 7.57 & 7.75 & 7.22 \\
\hline
\end{tabular}

\section{Uncertainty}

The uncertainty associated with the fractal parameters was difficult to estimate due to the empirical nature of the analysis. Errors in measurement from the SEM images were obscured in the image analysis, since the images were distorted somewhat during processing (as shown in Chapter 3) and the 'true' measurement was unknown. In 
addition, the use of empirical constants in the projected area analysis $\left(k_{a}\right.$ and $\alpha_{a}$ in Equation 3.2) likely introduced further uncertainty. These errors were further obscured when it was the slope and intercept of a line of best fit to the data that was of significance. Nevertheless, since the slope and intercept were determined from a line of best fit using least-squares regression, the uncertainties on the fractal parameters were assumed to be equivalent to the confidence intervals on the fits. Thus, these estimated uncertainties represent the spread in the data and are therefore estimates of precision error, which was assumed to be the dominant source of error for the sake of this analysis. This assumption may seem questionable, but the reader is reminded that the scatter correction term in the governing extinction equation (Equation 2.29) appears as $\left(1+\rho_{s a}\right)$. For a $\rho_{s a}$ value on the order of 0.3 , a $10 \%$ increase in uncertainty on $\rho_{s a, \lambda}$ results in a contribution of only $3 \%$ to the total uncertainty of $E(m)_{\lambda}$. Also, the additional uncertainty introduced by this assumption should be small in comparison to the overall uncertainty on $\rho_{s a, \lambda}$.

\subsubsection{Aggregate Size Distribution}

Normalized aggregate size distributions were found by fitting both log-normal and self-preserving distributions to histograms of aggregate size data from the SEM image analysis, as discussed in Section 3.3.5. The resulting best-fit parameters are listed in Table 4.5.

Table 4.5: Soot aggregate size distribution parameters for log-normal $\left(N_{g}, \sigma_{2 g}\right)$ and self-preserving $\left(M_{1}, \tau\right)$ distributions

\begin{tabular}{|c|c|c|c|c|c|c|c|c|c|c|c|c|}
\cline { 2 - 13 } \multicolumn{1}{c|}{} & A1 & B1 & B2 & C1 & C2 & C3 & D1 & D2 & D3 & E1 & E2 & E3 \\
\hline$N_{g}$ & 80.9 & 53.2 & 12.8 & 66.1 & 120 & 176 & 67.1 & 42.6 & 35.2 & 48.6 & 77.5 & 107 \\
\hline$\sigma_{2 g}$ & 3.39 & 3.10 & 5.06 & 3.27 & 4.23 & 3.40 & 3.01 & 4.20 & 3.33 & 3.53 & 3.04 & 2.63 \\
\hline$M_{1}$ & 89.9 & 64.7 & 17.3 & 78.2 & 112 & 155 & 77.2 & 57.5 & 46.4 & 62.7 & 75.9 & 121 \\
\hline$\tau$ & -0.31 & -0.31 & 0.75 & -0.27 & -0.14 & -0.58 & -0.48 & 0.24 & 0.01 & -0.02 & 0.58 & 0.13 \\
\hline
\end{tabular}




\section{Uncertainty}

The uncertainty on the distribution parameters is subject to the same difficulties as discussed above for the fractal parameters. In this case, the confidence limits on the fit parameters were much wider, giving further weight to the notion that this representation of the precision error dominates the bias error contribution to the total. Again in this case, the confidence limits given for the fit of the aggregate size histogram to the statistical distributions were used at face value, since they were assumed to be the dominant source of uncertainty on the distribution parameters. The uncertainties on both distributions were carried forward to better understand the effect of distribution choice on $\rho_{s a}$.

\subsubsection{Calculating $\rho_{s a}$ Results}

The calculation of scatter-to-absorption ratio was performed for all twelve conditions and for both log-normal and self-preserving distributions. Figure 4.6 shows results for the single condition A1 calculated using both log-normal and self-preserving distributions. Differences between the results using the two distributions are perhaps significant, although the uncertainty bars do overlap at all wavelengths. Furthermore, it can be seen that the uncertainties when using the self-preserving distribution are narrower than for the log-normal distribution - this difference is attributable to the quality of the data fitting, indicating that the self-preserving distribution provides a better fit to the SEM aggregate size data.

There is a trend of non-linear, though monotonic, decrease of $\rho_{s a, \lambda}$ with increasing wavelength. This trend is shown in Figure 4.6 for condition A1, but it was common to all conditions. Although the uncertainties overlap, scattering to absorption ratios calculated using the self-preserving distribution are slightly lower than those calcu- 


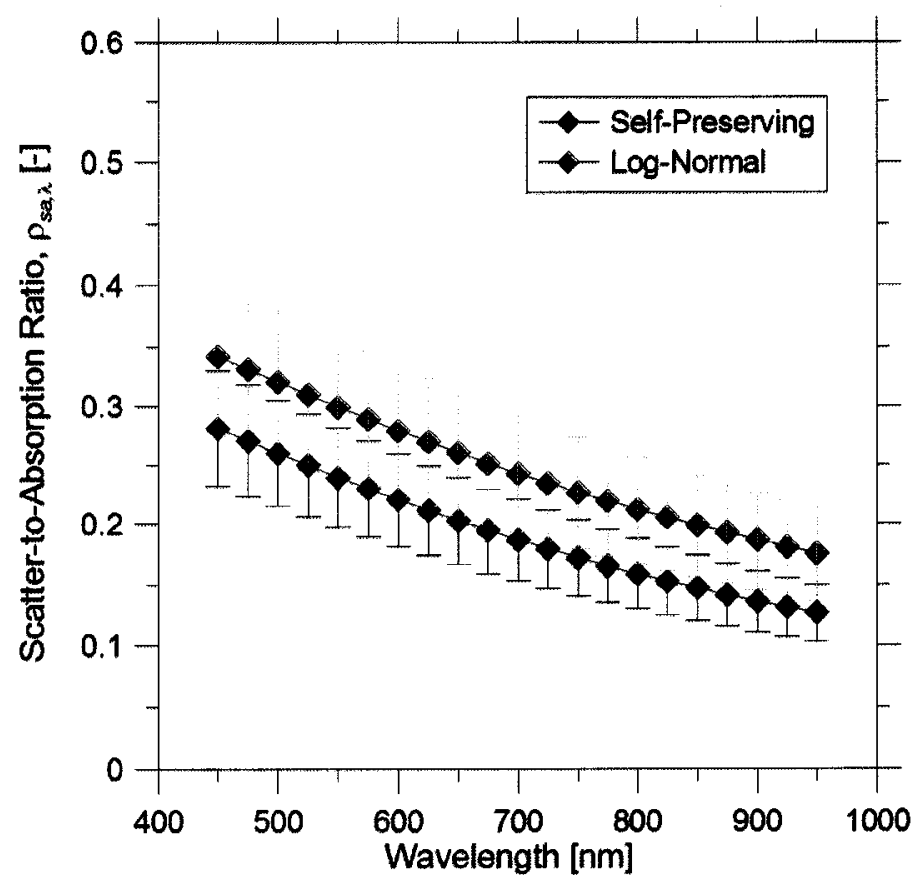

Figure 4.6: Scatter-to-absorption ratio for condition A1

lated with the more commonly used log-normal distribution, and this is consistent for all of the conditions tested.

Figure 4.7 compares the effect of dilution rate on $\rho_{s a, \lambda}$. For both distributions, condition D3 shows lower $\rho_{s a, \lambda}$ values than do conditions C3 and E3, indicating that there is no distinct trend in scattering to absorption ratio with dilution rate. Interestingly, there is also no distinct correlation between $\rho_{s a, \lambda}$ and the distribution parameters, in particular with the mean value of $N$. Since scattering increases with $N^{2}$, and absorption only with $N$, it would be reasonable to expect $\rho_{s a, \lambda}$ to increase proportionally to $N$. However, that trend is not observed here (C3 having the highest mean value of $N$, and D3 the lowest). The absence of correlation is inconclusive, however, since there is a significant amount of uncertainty present in these results.

Figures 4.8 and 4.9 show $\rho_{s a, \lambda}$ results for all conditions calculated using log-normal and self-preserving distributions. For both cases, the spread in data among conditions 
is small compared to the error bars. The overall variability among conditions is similar for both distributions, though the standard deviation among conditions at a given wavelength were $20-25 \%$ smaller for the self-preserving case than for the log-normal case. However, consistency is not expected between soot generated at different burner conditions, so this is not necessarily a good criterion on which to judge the suitability of the distribution. Also, the self-preserving distribution is more cumbersome to apply, so it is not clear from this work whether the additional complexity is warranted.

\section{Uncertainty on $\rho_{s a, \lambda}$}

The scatter to absorption ratio is dependent on many factors, and the form of the dependence is too complex (as outlined in Chapter 2) to apply the bias limit determination method above. Instead, a parametric analysis was performed, where each parameter was varied to its bias limits and the effect on the scatter to absorption ratio was observed. In addition, a blanket value of $10 \%$ uncertainty was added in order to capture uncertainties inherent in the use of the RDG-PFA approximation, as discussed in Section 2.4.5. The experimental parameters that contribute to $\rho_{s a}$ are the fractal parameters $D_{f}$ and $k_{g}$, the primary particle size $d_{p}$, and the fits of size data to statistical distributions $\left(N_{g}\right.$ and $\sigma_{2 g}$ for the log-normal distribution, and $M_{1}$ and $\tau$ for self-preserving). The uncertainties of these parameters are listed in Table 4.6 for sample case $\mathrm{C} 3$. The analysis was repeated for all wavelengths and for fits to both log-normal (LN) and self-preserving (SP) distributions. The total uncertainty was determined by combining the contributions of each parameter in quadrature, as before. 


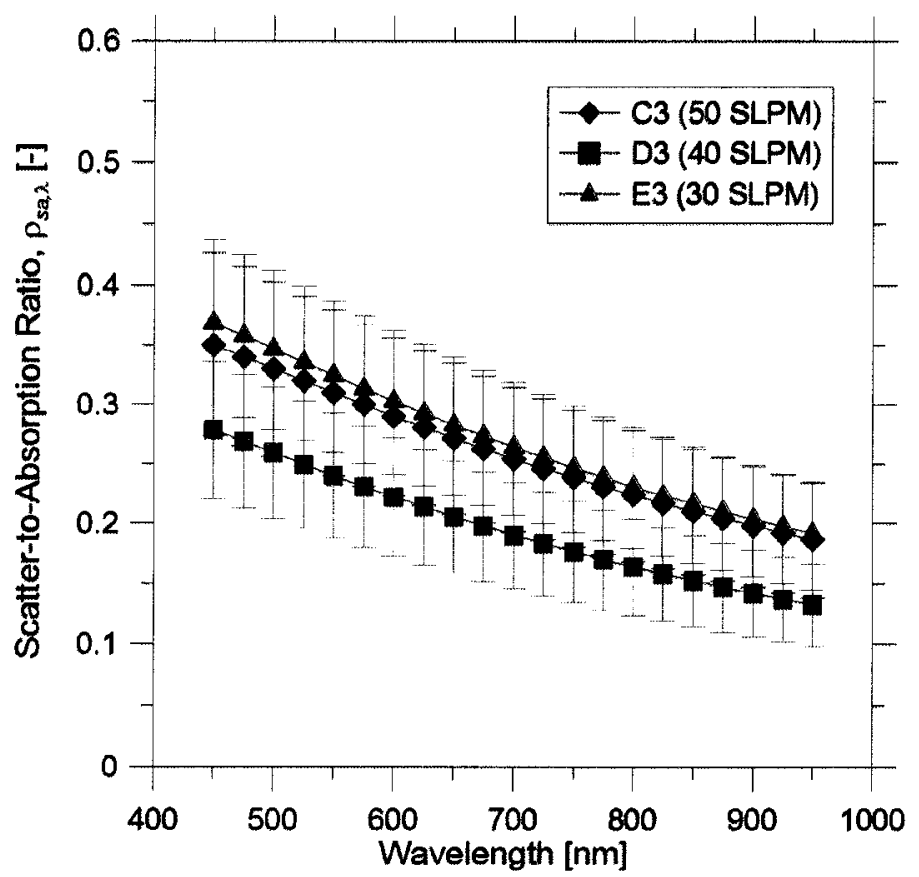

(a) Log-normal distribution

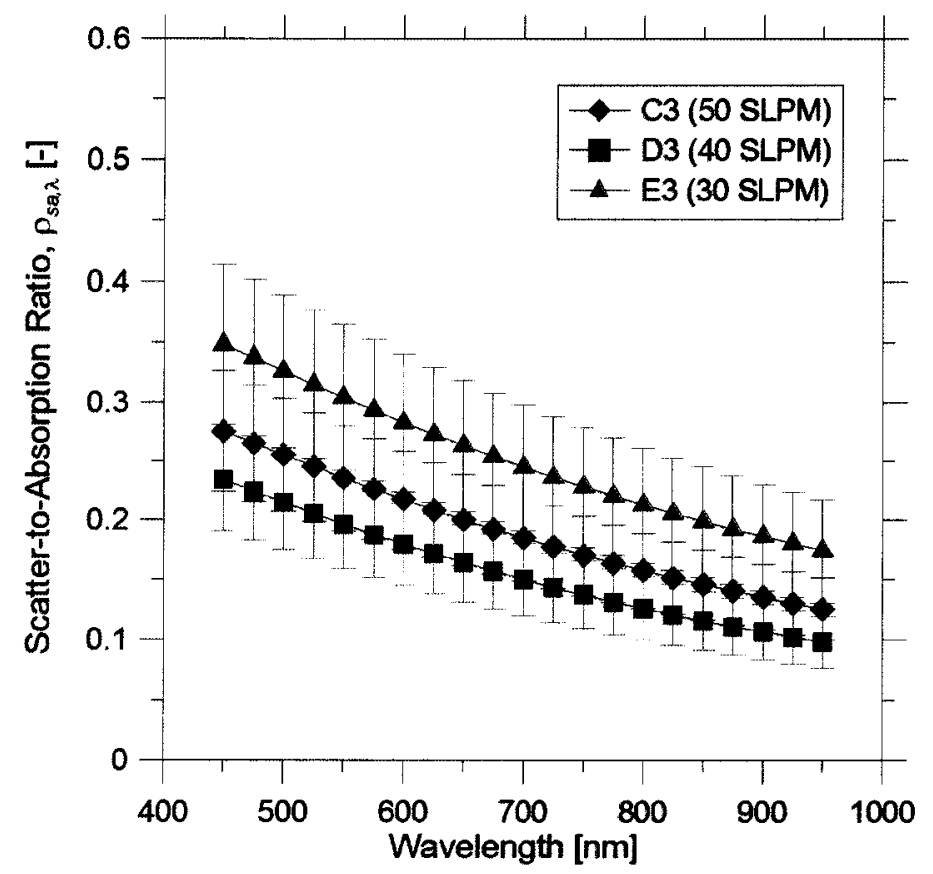

(b) Self-preserving distribution

Figure 4.7: Scatter-to-absorption ratio data comparing dilution rates 


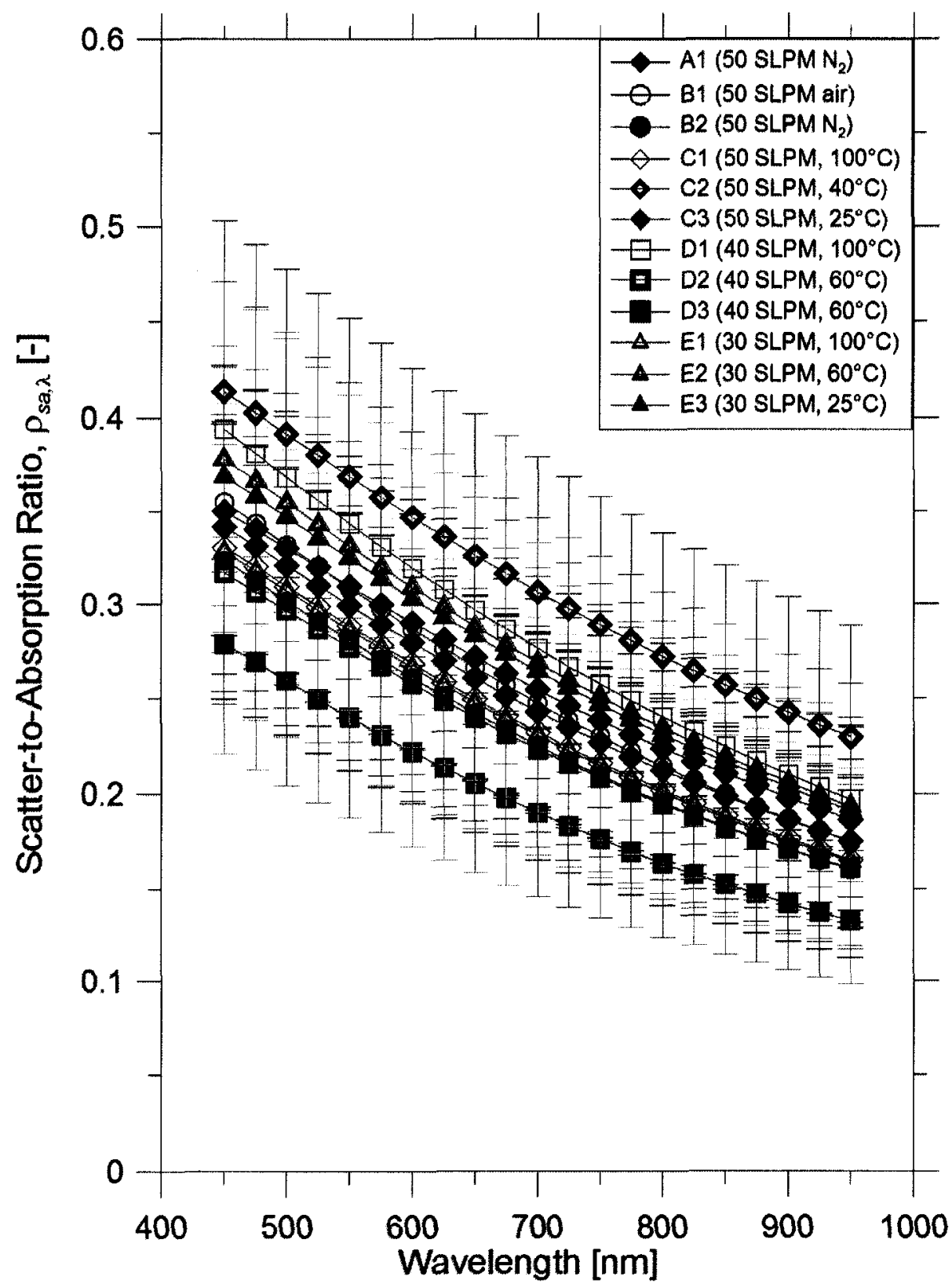

Figure 4.8: Scatter-to-absorption ratio results for all conditions, calculated using the log-normal distribution (refer to Table 3.1 on page 44 for further description of the burner conditions) 


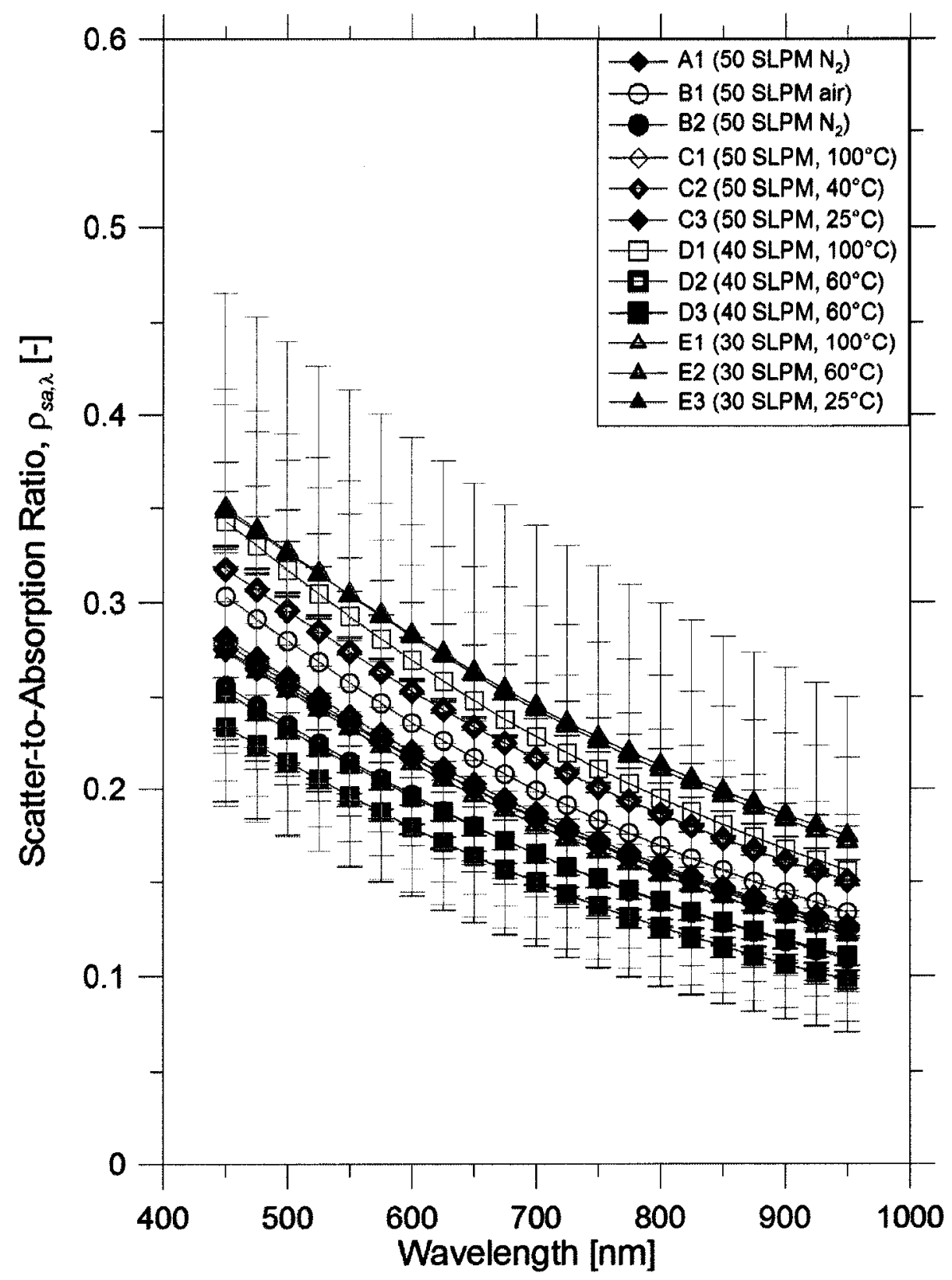

Figure 4.9: Scatter-to-absorption ratio results for all conditions, calculated using the self-preserving distribution (refer to Table 3.1 on page 44 for further description of the burner conditions) 
Table 4.6: The 95\% confidence limits of $\rho_{s a}$ parameters for case C3

\begin{tabular}{|c|c|c|c|c|}
\hline & Best & Lower & Upper \\
\hline & $D_{f}$ & 1.69 & 1.66 & 1.72 \\
\hline & $k_{f}$ & 9.40 & 8.49 & 10.3 \\
\hline & $d_{p}[\mathrm{~nm}]$ & 33.2 & 31.0 & 35.4 \\
\hline $\mathrm{LN}$ & $N_{g}$ & 176 & 117 & 234 \\
\hline & $\sigma_{2 g}$ & 3.41 & 2.81 & 4.00 \\
\hline $\mathrm{SP}$ & $\overline{M_{1}}$ & 155 & 129 & 182 \\
\hline & $\tau$ & -0.59 & -0.83 & -0.34 \\
\hline
\end{tabular}

\subsection{Soot Absorption Function}

Data from the preceding diagnostics were combined according to Equation (2.29) to quantify the wavelength-dependent soot absorption function $E(m)_{\lambda}$, using scatter corrections calculated with both log-normal and self-preserving distributions. Figure 4.10 shows $E(m)_{\lambda}$ results for condition A1. Note that the discontinuity between values at lower and higher wavelengths is due to the discontinuity in the two sets of extinction measurements, as discussed in Section 4.2. The wavelength trend seen in this figure, where there was little variation in $E(m)_{\lambda}$ with the visible wavelengths (up to $700-750 \mathrm{~nm}$ ), and a decrease with increasing wavelength in the infrared, was common to all conditions. Also for all cases, the analysis using the log-normal distribution produced values slightly lower than when using the self-preserving distribution. The discrepancies between the two approaches were generally within the uncertainty limits.

Figure 4.11 compares results for the three dilution rates in which the additional dilution air was injected at room temperature. The apparent trend of $E(m)$ increasing proportionally to dilution rate seen in this figure is somewhat misleading, as the condition C3 is somewhat of an outlier; this trend is not found when comparing the effect of dilution rate in the 1 - and 2- series. 


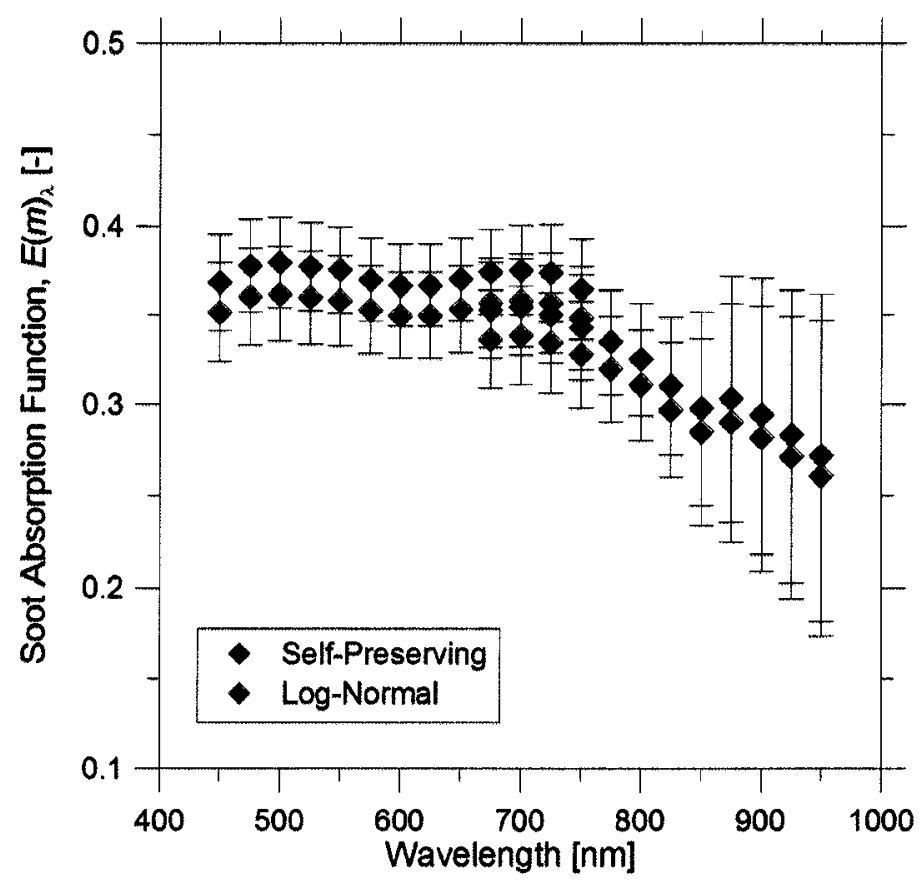

Figure 4.10: Soot absorption function results for condition A1

Figures 4.12 and 4.13 show $E(m)_{\lambda}$ results for all conditions, using $\rho_{s a, \lambda}$ values calculated using the log-normal and the self-preserving distribution, respectively. These figures demonstrate that the wavelength trend discussed above, where there is little variation in $E(m)_{\lambda}$ across the visible wavelengths and a monotonic decrease with increasing wavelength in the infrared, is common to all conditions and for both distributions. In fact, the differences seen as a result of the choice of distribution are small compared to the uncertainty in the measurements.

\section{Uncertainty}

The determination of $E(m)_{\lambda}$ is a calculation rather than a measurement, requiring only error propagation without the addition of further uncertainty. With the uncertainties on all the required variables known, the total uncertainty on the absorption function $E(m)_{\lambda}$ can be determined by combining individual contributions in quadra- 


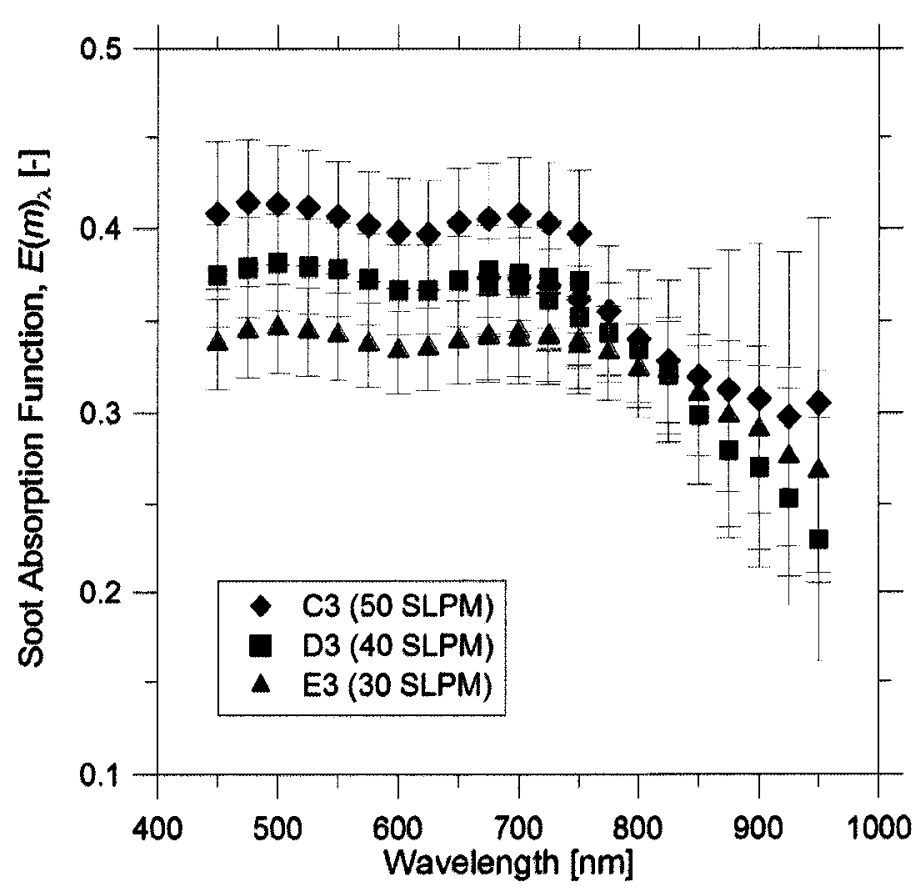

(a) Using the log-normal distribution

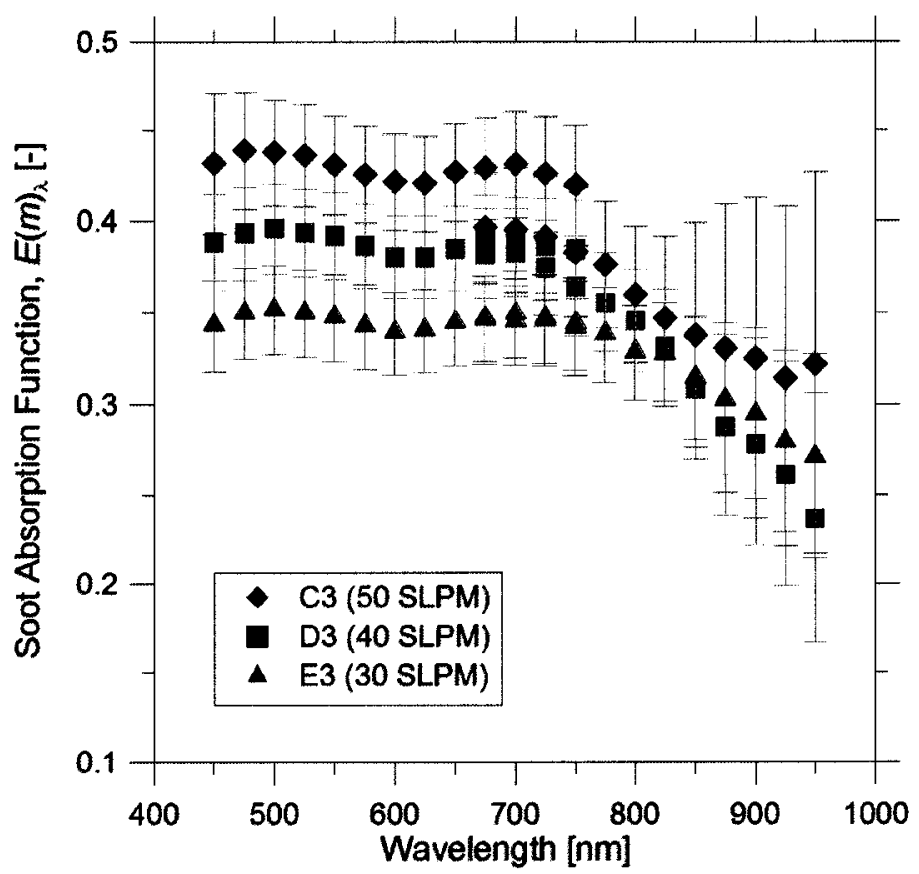

(b) Using the self-preserving distribution

Figure 4.11: Soot absorption function results, comparing dilution rates 


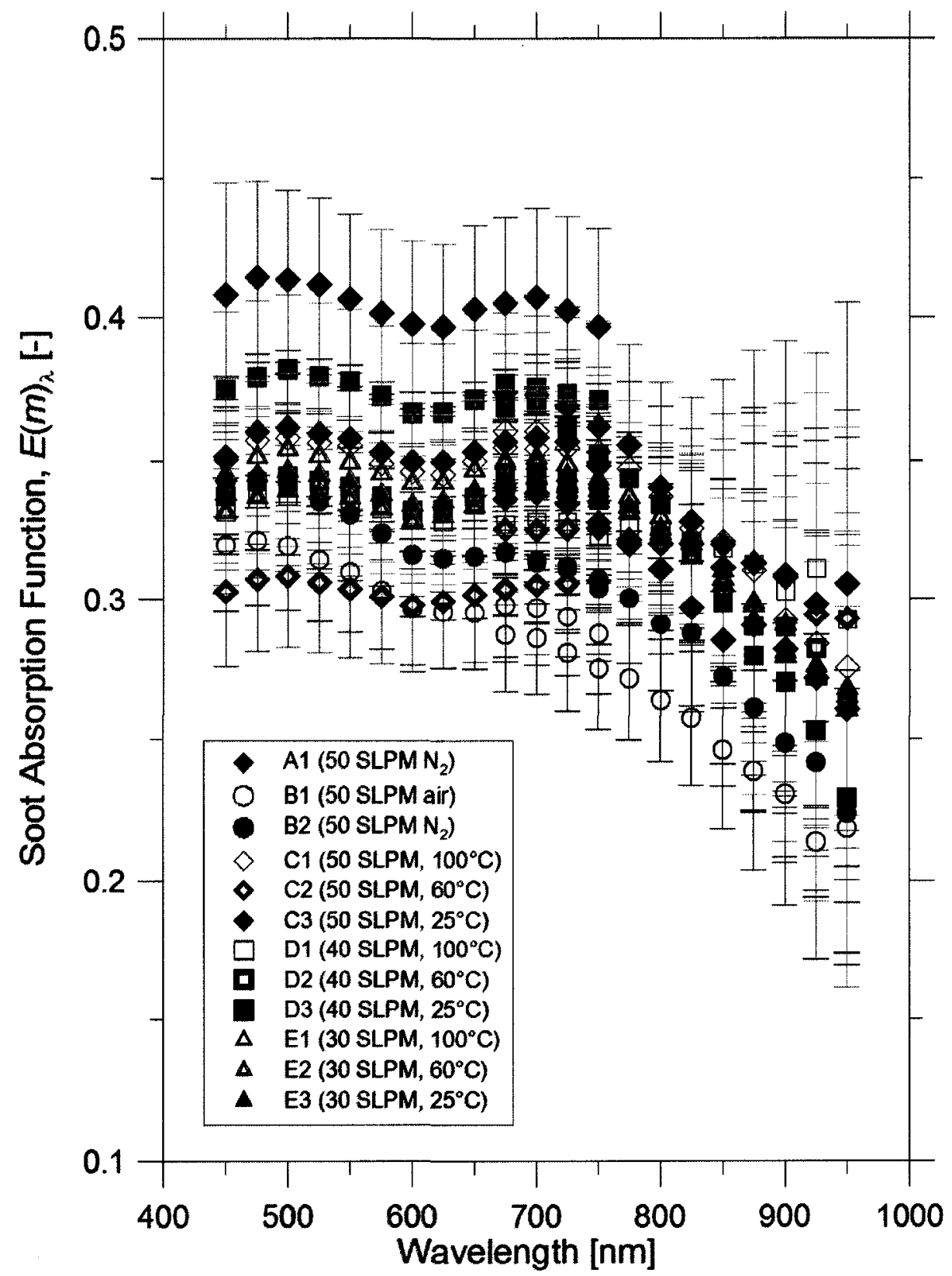

Figure 4.12: Soot absorption function results for all conditions, with $\rho_{s a, \lambda}$ calculated using log-normal distribution (refer to Table 3.1 on page 44 for further description of the burner conditions) 


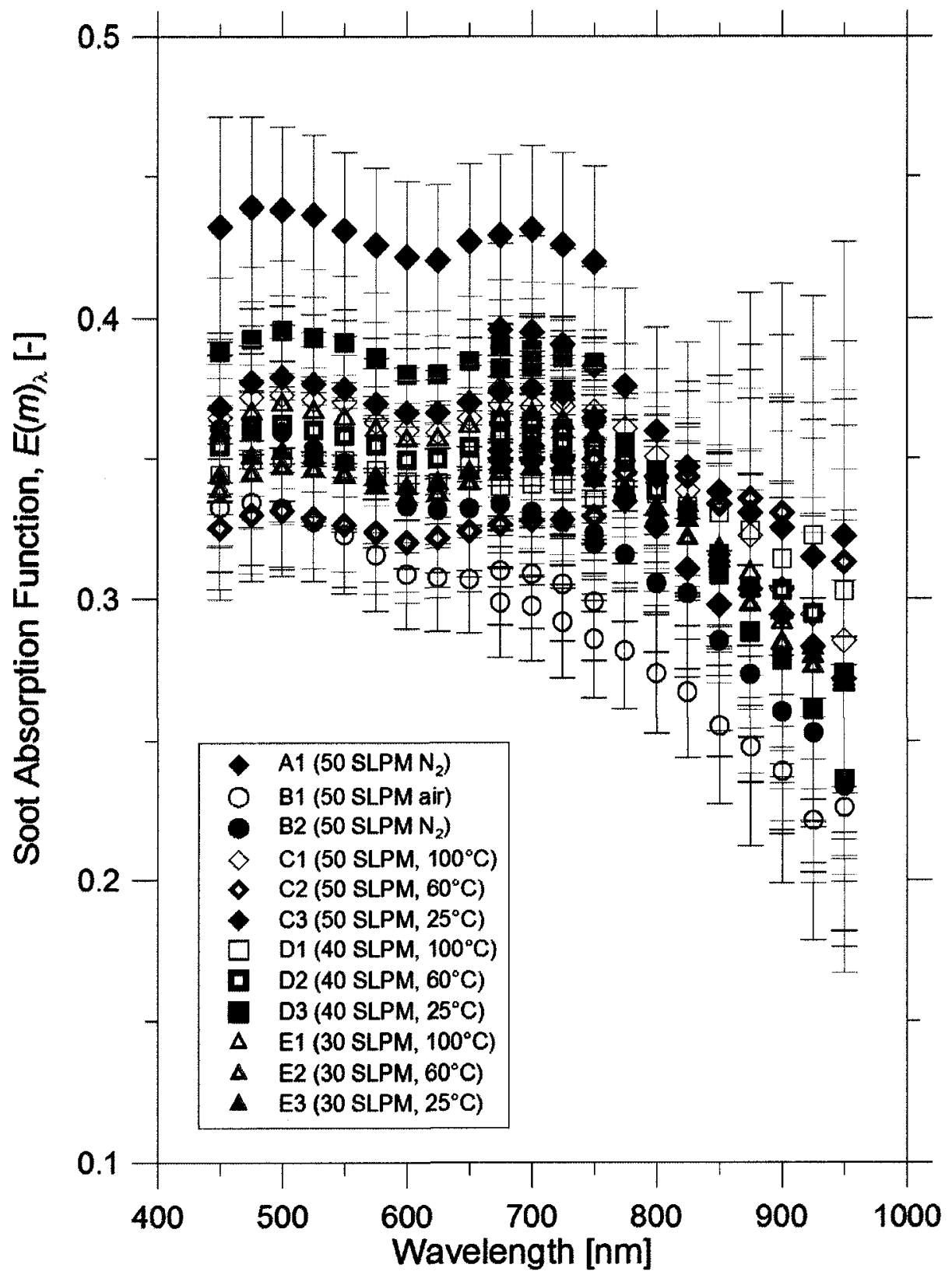

Figure 4.13: Soot absorption function results for all conditions, with $\rho_{s a, \lambda}$ calculated using self-preserving distribution (refer to Table 3.1 on page 44 for further description of the burner conditions) 
ture as before, shown in Equation (4.3).

$$
\frac{U_{E(m)_{\lambda}}}{E(m)_{\lambda}}=\sqrt{\left(\frac{U_{K_{e x t, \lambda}}}{K_{e x t, \lambda}}\right)^{2}+\left(\frac{U_{\rho_{s a, \lambda}}}{1+\rho_{s a, \lambda}}\right)^{2}+\left(\frac{U_{f_{v}}}{f_{v}}\right)^{2}}
$$

\subsection{Soot Volume Fractions}

Laser-induced incandescence data were analyzed using the wavelength-independent value of $E(m)=0.4$, given by Snelling et al. [2004] (the value currently used within the combustion group at NRC-ICPET), as well as the results of the present study given in the previous section. These results can be seen in Figures 4.14 and 4.15, respectively. Measured LII data is plotted as a function of laser fluence used to heat the particles, which, at least for the case of the cooled soot being measured here, is observed to have a strong influence on measured soot volume fraction. While deeper analysis and discussion for the possible reasons of this trend are beyond the scope of this thesis, the data and discussion on $E(m)_{\lambda}$ and $\rho_{s a, \lambda}$ dependencies could support deeper understanding of this behaviour.

The peak values of soot volume fraction from the LII measurements using different $E(m)_{\lambda}$ functions were compared to the soot volume fraction obtained by gravimetric analysis, as shown in Figure 4.16. In nearly all cases, the LII soot volume fraction obtained using the present $E(m)_{\lambda}$ values are closer to the gravimetric result than are the LII results with a flat $E(m)=0.4$. Moreover, the analysis using the flat $E(m)=$ 0.4 greatly underpredicted the soot volume fraction for the underventilated " $B$ " series, represented by the two rightmost data points in the figure. Interestingly, however, the analysis using the present condition-specific results produced more scatter in the data (i.e., lower precision), despite the values being closer to the gravimetric result (i.e., greater accuracy). 


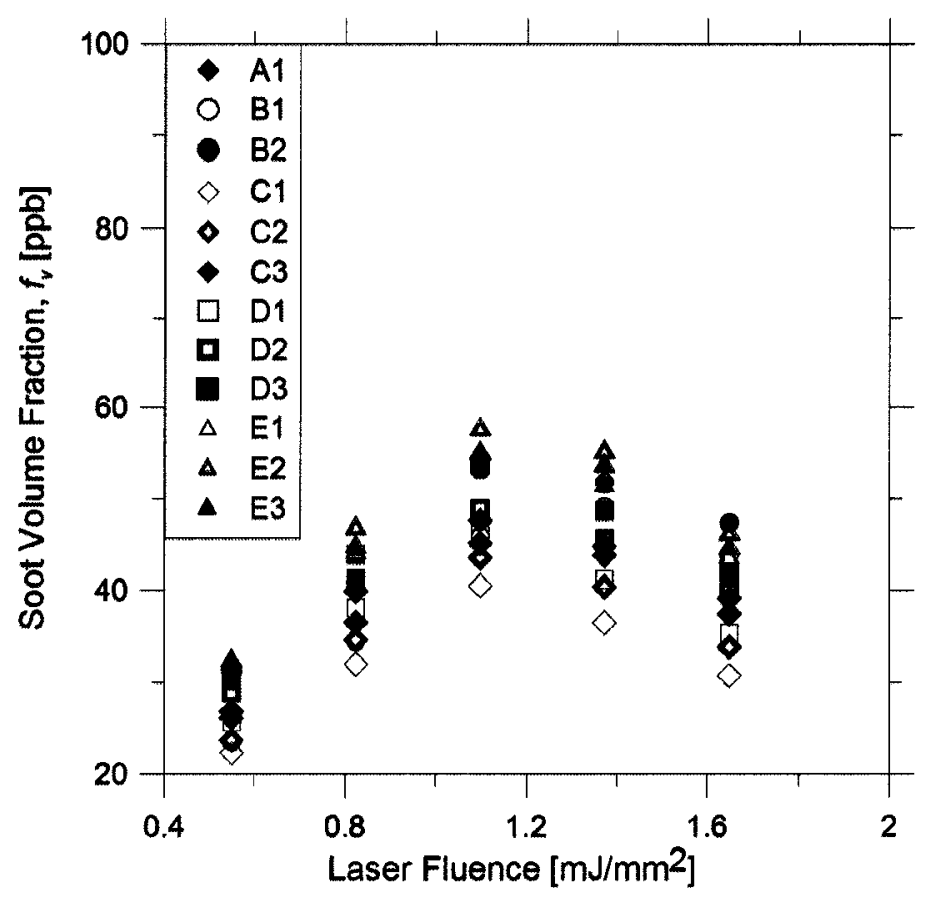

Figure 4.14: LII soot volume fraction versus laser fluence, using flat $E(m)$ of 0.4 (refer to Table 3.1 on page 44 for further description of the burner conditions)

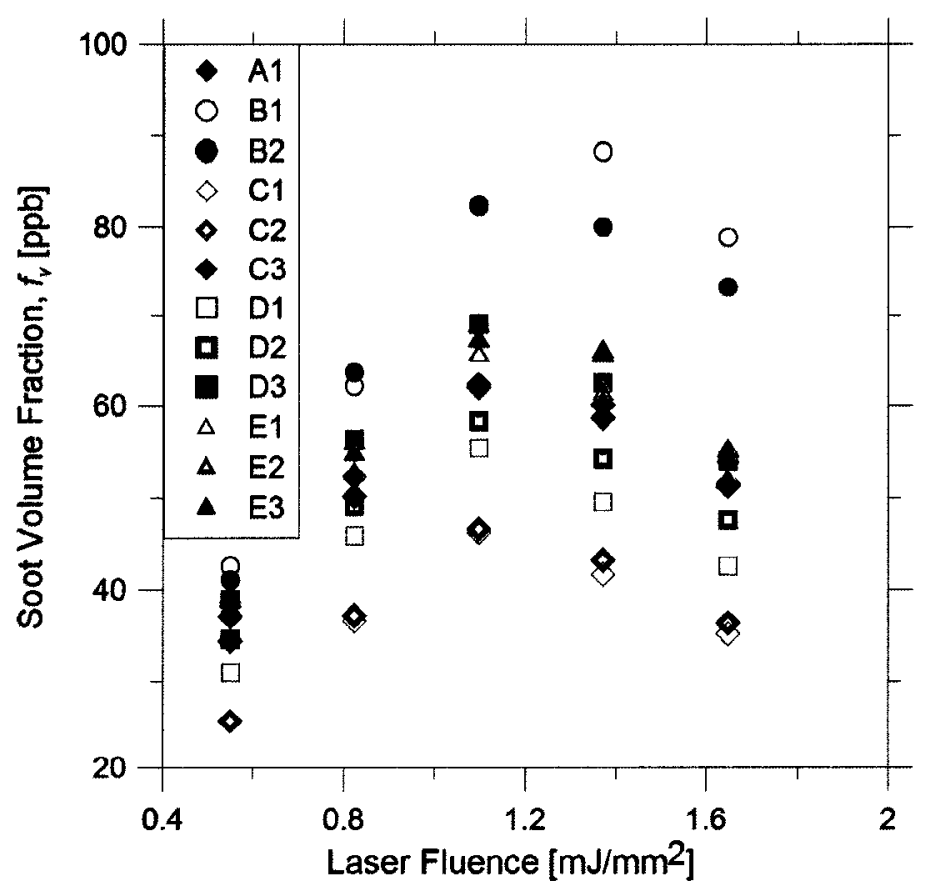

Figure 4.15: LII soot volume fraction versus laser fluence, using $E(m)_{\lambda}$ values from the present work (refer to Table 3.1 on page 44 for further description of the burner conditions) 


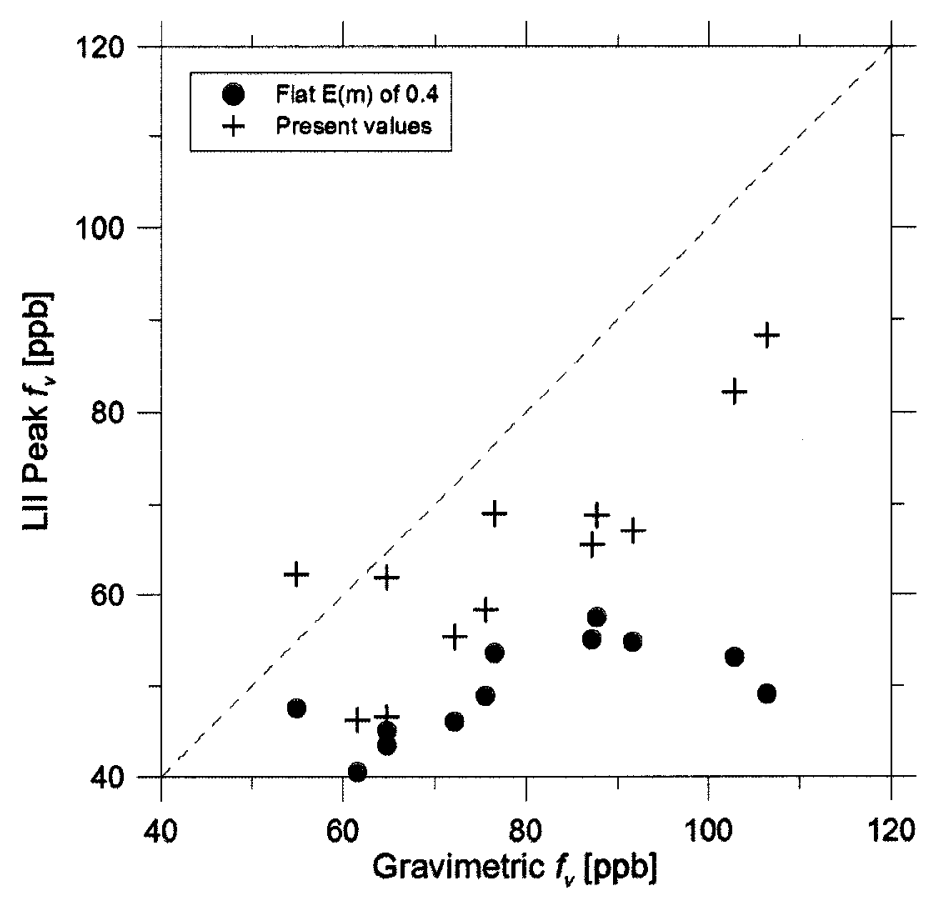

Figure 4.16: Comparison of peak soot volume fractions found by LII and gravimetric analysis 


\section{Chapter 5}

\section{Discussion}

\subsection{Effect of Distribution Choice}

In the context of overall uncertainties, the calculated $E(m)_{\lambda}$ data presented in Figures 4.12 and 4.13, using the log-normal and the self-preserving distributions, showed little difference. However, data derived using the self-preserving distribution had slightly narrower confidence limits, thereby reducing the uncertainty associated with the scatter correction. The self-preserving distribution also has a theoretical basis in aggregating systems, as cited in Section 1.1.2, giving it further advantage over the log-normal distribution. Because of these advantages and because the data are quite similar between the two, this discussion will focus solely on the results found using the self-preserving distribution.

\subsection{Comparison Among Conditions}

The experimentally derived values of $E(m)_{\lambda}$, shown in Figure 4.13 for the twelve burner conditions investigated in this work, were plotted against several relevant pa- 
rameters specific to the experiment in an attempt to better understand the variability. These parameters included the measured physical or structural quantities of the soot $\left(D_{f}, k_{g}, d_{p}, N, R_{g}, f_{v}\right)$, indirect quantities relating to soot formation calculated from experiment operating parameters (exhaust stream residence time, characteristic aggregation time), other experimental parameters (wavelength, dilution ratio), as well as several combinations of the above. A subset of these plots is available in Appendix C. However, correlation was found with only one, somewhat obvious, parameter: the wavelength of light.

The lack of additional correlation is perhaps unsurprising considering the relatively small variation in the $E(m)_{\lambda}$ results among conditions at a given wavelength; all measurements of $E(m)_{\lambda}$ fell within $20 \%$ of the mean, while the standard deviation among conditions was only $8 \%$ at $450 \mathrm{~nm}$, growing to a maximum of just over $11 \%$ at $950 \mathrm{~nm}$. The $E(m)_{\lambda}$ values calculated for condition C3 were a significant outlier, without which the data fell within $10 \%$ of the mean at the shorter wavelengths, with a standard deviation as low as $5 \%$. The consistency in the $E(m)_{\lambda}$ data persisted despite large variations in several of the input parameters, including a nearly factor of two variation in the dilution ratio, which, coupled with the dilution air temperature variation, resulted in a $50 \%$ difference in the exhaust residence time.

The lack of variability in $E(m)_{\lambda}$ among the tested conditions suggests that, within uncertainty limits, the parameters varied have little effect on the resultant optical properties of soot. Negligible differences were found with varying dilution rate (which affects exhaust residence time and temperature history), with dilution temperature (which also affects residence time and temperature history), or with dilution gas (which would reflect post-flame oxidation effects). If the measurements made for the twelve burner conditions studied here are considered repeated measurements of the same values, the spread in their results can be seen as a measure of the precision 


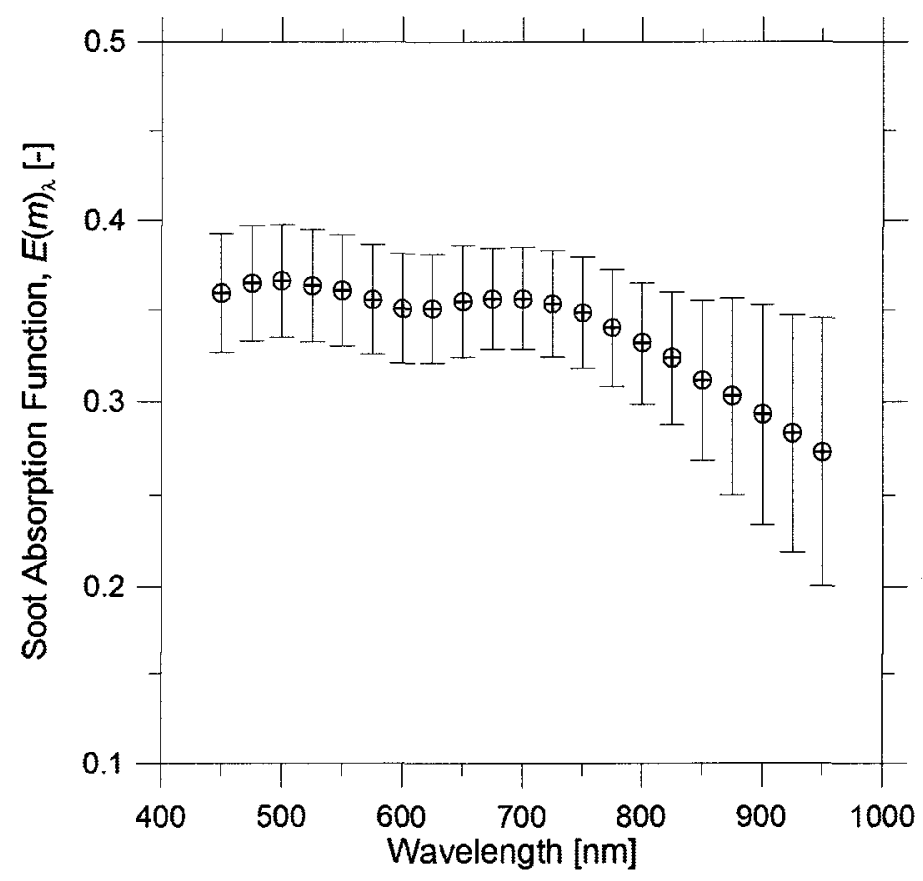

Figure 5.1: $E(m)$ results, treating the various conditions as repeated measurements

error. The results of such a treatment can be seen in Figure 5.1.

The relatively narrow uncertainty bars seen in the figure suggest that it could be reasonable to treat the twelve conditions as repeated measurements. Under this assumption, a wavelength-dependent soot absorption function could be defined for more generalized use at a range of conditions. Figure 5.1 also serves to clarify the wavelength dependence of $E(m)$ seen in the present work, the consistency of which is indicative of a universal trend. A discussion of the spectral variation of $E(m)$ is presented in the following section.

\subsubsection{Variation with Wavelength}

The variation of the absorption function with wavelength is important for optical diagnostics that rely on data from more than a single wavelength, such as two-colour pyrometry and time -resolved laser-induced incandescence. Indeed, this was the mo- 
tivation for acquiring spectrally-resolved data in this work. The ratio of $E(m)$ values between wavelengths is sometimes referred to as the relative $E(m)$, which we shall denote as $\tilde{E}(m)$. Its importance in the LII diagnostic was seen in Equation (1.13), where $\tilde{E}(m)$ is used in determining the particle temperature.

Figure 5.2 shows calculated results of $\tilde{E}(m)$ for all conditions, where each condition is normalized with respect to a reference value:

$$
\tilde{E}(m)_{\lambda}=\frac{E(m)_{\lambda}}{E(m)_{r e f}}
$$

Some cases show a significant discontinuity between the lower-wavelength and upperwavelength extinction data, so each wavelength range was normalized separately. The results were normalized both by the maximum value of the set in (a), and by the value at a fixed wavelength of $700 \mathrm{~nm}$ in (b). Also included in the figures are $6^{\text {th }}$-order polynomial fits to the condition-averaged data. This functional form has no known physical basis and was chosen simply as the lowest order fit that captured the visible trend in the data.

Figure 5.2(b) shows that for all conditions, the soot absorption function displays the same general dependence on wavelength. Agreement is excellent at the lower wavelengths (1-3\% standard deviations) and results diverge only slightly at the higher wavelengths (with standard deviations less than $4 \%$ below $800 \mathrm{~nm}$, and as high as $10 \%$ above). Data for the two conditions in the underventilated "B" series are possible exceptions, showing a difference in slope at the lower wavelengths compared to the other conditions. That this difference in slope was seen only in the tests done at underventilated conditions could indicate that flame condition has some impact on $\tilde{E}(m)$.

As for the source of this difference, recall that three variables appear in the cal- 
culation of $E(m)$ : scatter-to-absorption ratio, soot volume fraction, and volumetric extinction coefficient. Referring back to Figures 4.8 and 4.9, no abnormalities were seen in the scatter correction for these two conditions, which would have reflected any significant differences in the physical structure of the soot. The soot volume fraction is not a wavelength-dependent property, thus responsibility for the slope change rests with the extinction measurements. Indeed, inspection of Figure 4.3 does reveal a difference in slope in the B-series at wavelengths below $600 \mathrm{~nm}$ compared to the other conditions. However, the reason for this is unclear at this point, and could be something worth investigating in future work.

\subsection{Dimensionless Extinction}

Several authors [e.g. Dobbins and Megaridis, 1991, Choi et al., 1995, Krishnan et al., 2000] have reported a dimensionless extinction constant as the result of their extinction measurements. This dimensionless constant, denoted $K_{e}$ here, is formulated as follows:

$$
\tau=\frac{I_{\lambda}}{I_{\lambda 0}}=\exp \left[\frac{-K_{e, \lambda}\left(1+\rho_{s a, \lambda}\right) f_{v} L}{\lambda}\right]
$$

This equation, when compared to Equation (1.15), results in the dimensionless extinction constant being related to the volumetric extinction coefficient by the following expression:

$$
K_{e, \lambda}=\frac{K_{e x t, \lambda} \lambda}{f_{v}}
$$

Equation (5.3) has a similar form to Equation (2.29), used to calculate $E(m)_{\lambda}$, only without the dependence on $\rho_{s a, \lambda}$ for the scatter correction. Since a fair amount of uncertainty is introduced by the scatter correction, it is perhaps a useful exercise to re-cast the present results in terms of the dimensionless extinction constant. As with 


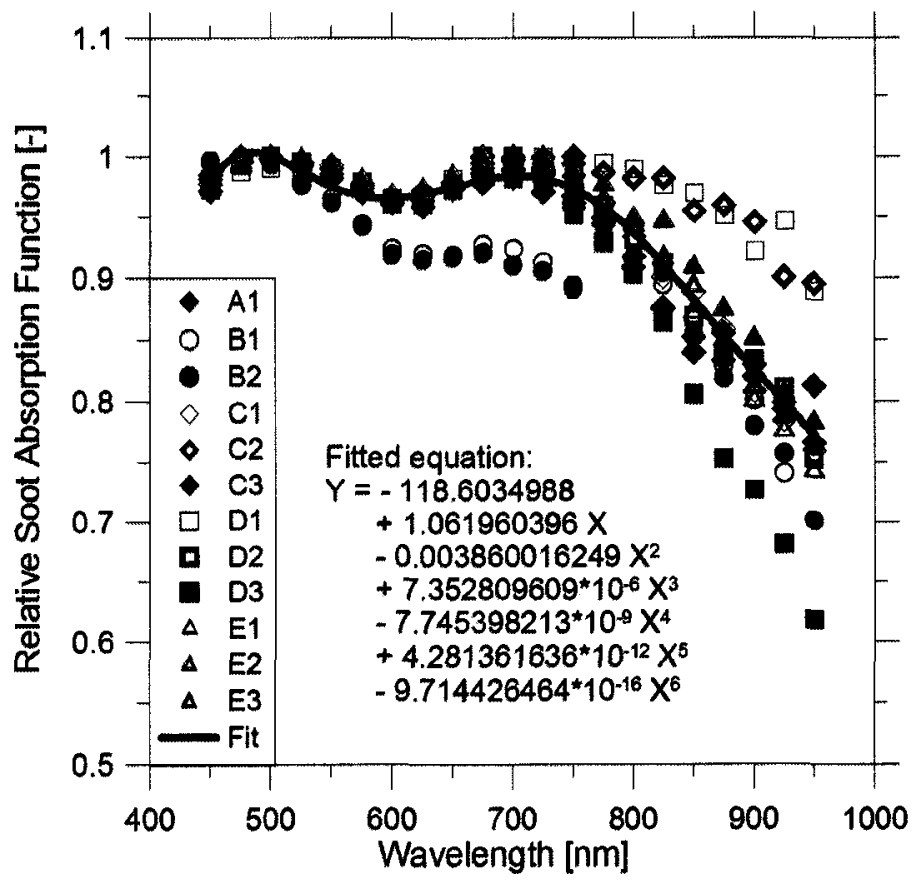

(a)

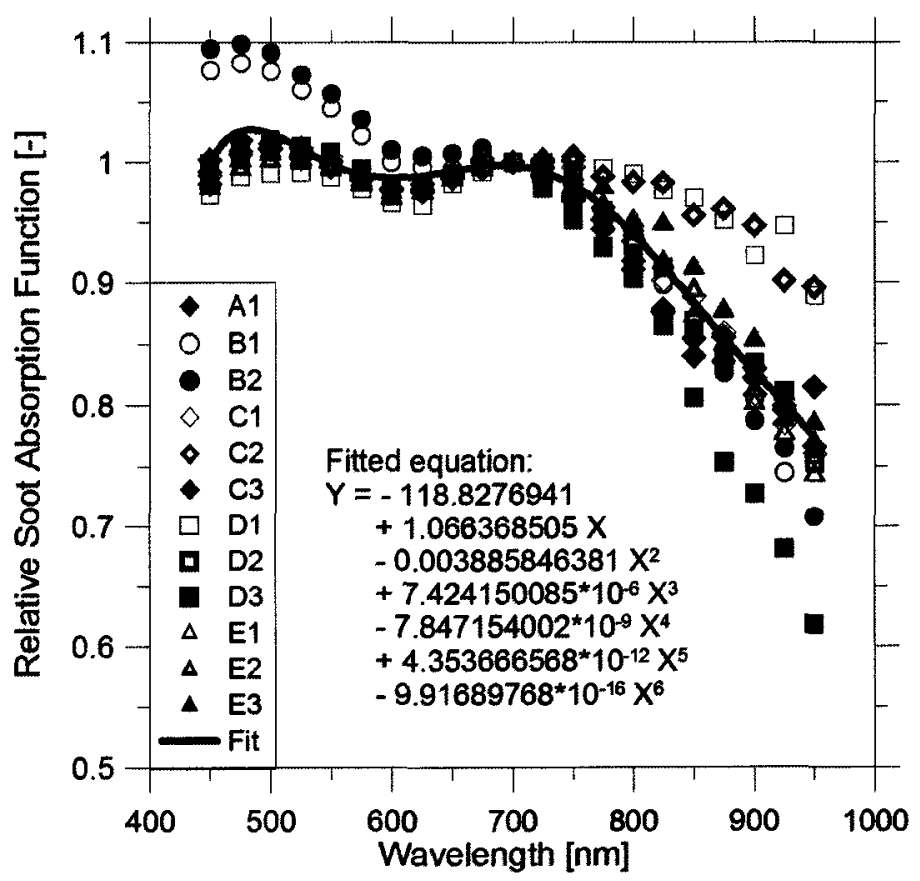

(b)

Figure 5.2: $\tilde{E}(m)$ results for all conditions, each wavelength range normalized by (a) the maximum value in that set and (b) the value corresponding to a wavelength of $700 \mathrm{~nm}$ in that set (refer to Table 3.1 on page 44 for further description of the burner conditions) 
the $E(m)$ results, $K_{e}$ data were plotted against many variables in an attempt to find trends. However, once again, the only correlation found was with wavelength. Figure 5.3 shows $K_{e, \lambda}$ results for the twelve burner conditions, plotted against wavelength. Again, C3 is the outlier, otherwise the results are rather consistent. Indeed, the standard deviation in the results ranges from approximately $7 \%$ at $450 \mathrm{~nm}$ (less than $4 \%$ if $\mathrm{C} 3$ is excluded) to around $12 \%$ at $950 \mathrm{~nm}$, where uncertainty is significantly greater.

\subsection{Mass Absorption Cross-section}

Another representation of the absorption properties of particulate matter is the socalled mass absorption cross-section (MAC), which is used, for example, in the atmospheric particulate field [Bond and Bergstrom, 2006]. MAC represents the massnormalized absorption cross-section of particulate aerosol, which is found as follows [e.g. Schnaiter et al., 2003, Dobbins et al., 1994]:

$$
\mathrm{MAC}=\frac{6 \pi}{\lambda \rho_{s}} E(m)_{\lambda}
$$

Substituting Equation (2.29) into Equation (5.4), the following result is obtained:

$$
\mathrm{MAC}=\frac{K_{e x t, \lambda}}{\rho_{s}\left(1+\rho_{s a}\right) f_{v}}
$$

where $\rho_{s}$ is the mass density of soot. Figure 5.4 shows the data obtained in the present work in terms of MAC, plotted against wavelength. The results are similar: relatively little variation is seen among tested conditions (standard deviations of less than $9 \%$ at all wavelengths) and again condition $\mathrm{C} 3$ is the outlier. Removing data from this condition results in standard deviations among conditions as low as $5 \%$ at 


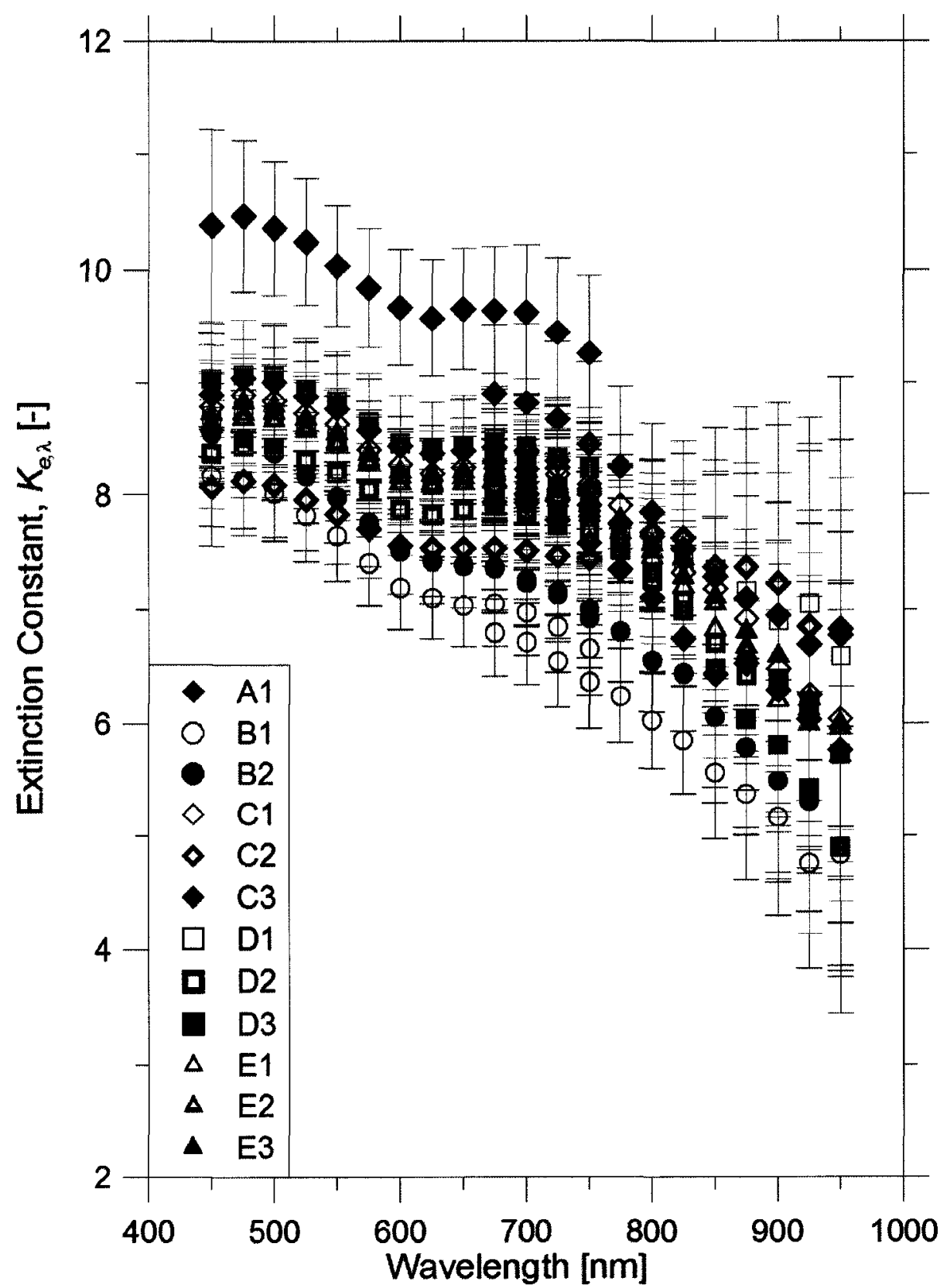

Figure 5.3: Dimensionless extinction constant versus wavelength for all conditions (refer to Table 3.1 on page 44 for further description of the burner conditions) 
Table 5.1: Summary of condition-averaged results

\begin{tabular}{|c|c|c|c|c|c|c|c|c|}
\hline$\lambda[\mathrm{nm}]$ & $\rho_{s a}$ & $U_{\rho_{s a}}$ & $E(m)$ & $U_{E(m)}$ & $K_{e}$ & $U_{K_{e}}$ & MAC $\left[\frac{m^{2}}{g}\right]$ & $U_{\mathrm{MAC}}\left[\frac{m^{2}}{g}\right]$ \\
\hline \hline 450 & 0.29 & 0.06 & 0.35 & 0.03 & 8.7 & 0.6 & 8.0 & 0.7 \\
\hline 500 & 0.27 & 0.06 & 0.36 & 0.03 & 8.8 & 0.6 & 7.3 & 0.6 \\
\hline 550 & 0.25 & 0.06 & 0.35 & 0.03 & 8.5 & 0.6 & 6.5 & 0.5 \\
\hline 600 & 0.23 & 0.05 & 0.34 & 0.03 & 8.1 & 0.6 & 5.8 & 0.5 \\
\hline 650 & 0.21 & 0.05 & 0.35 & 0.03 & 8.1 & 0.6 & 5.4 & 0.4 \\
\hline 700 & 0.20 & 0.05 & 0.35 & 0.03 & 8.0 & 0.5 & 5.1 & 0.4 \\
\hline 750 & 0.18 & 0.05 & 0.34 & 0.03 & 7.8 & 0.6 & 4.7 & 0.4 \\
\hline 800 & 0.17 & 0.04 & 0.33 & 0.03 & 7.3 & 0.7 & 4.2 & 0.4 \\
\hline 850 & 0.15 & 0.04 & 0.31 & 0.04 & 6.8 & 0.9 & 3.7 & 0.5 \\
\hline 900 & 0.14 & 0.04 & 0.29 & 0.06 & 6.3 & 1.3 & 3.7 & 0.7 \\
\hline 950 & 0.13 & 0.04 & 0.27 & 0.07 & 5.8 & 1.5 & 3.3 & 0.8 \\
\hline
\end{tabular}

the shorter wavelengths. The total uncertainty is less than $10 \%$ at wavelengths of 800 $\mathrm{nm}$ and below.

\subsection{Comparison to Literature}

As discussed above, the variability in the results among conditions is small compared to the variation in input parameters and to the propagated uncertainty. Thus, for comparison purposes, the obtained values have been averaged across all tested conditions and summarized in Table 5.1.

In Figure 5.5, the condition-averaged $E(m)_{\lambda}$ results seen in Figure 5.1 are plotted alongside the values from literature seen in Figure 1.2. Notwithstanding the wide spread of results reported in the literature, the present results show consistency with several previous studies. In particular, the $E(m)_{\lambda}$ results of Dobbins et al. [1994] and Schnaiter et al. [2003] show similar magnitudes, and results of Krishnan et al. [2000] and Snelling et al. [2004] compare favourably in terms of spectral variation $(\tilde{E}(m))$. The results of Köylü and Faeth [1996] at the lower wavelengths were also in reason- 


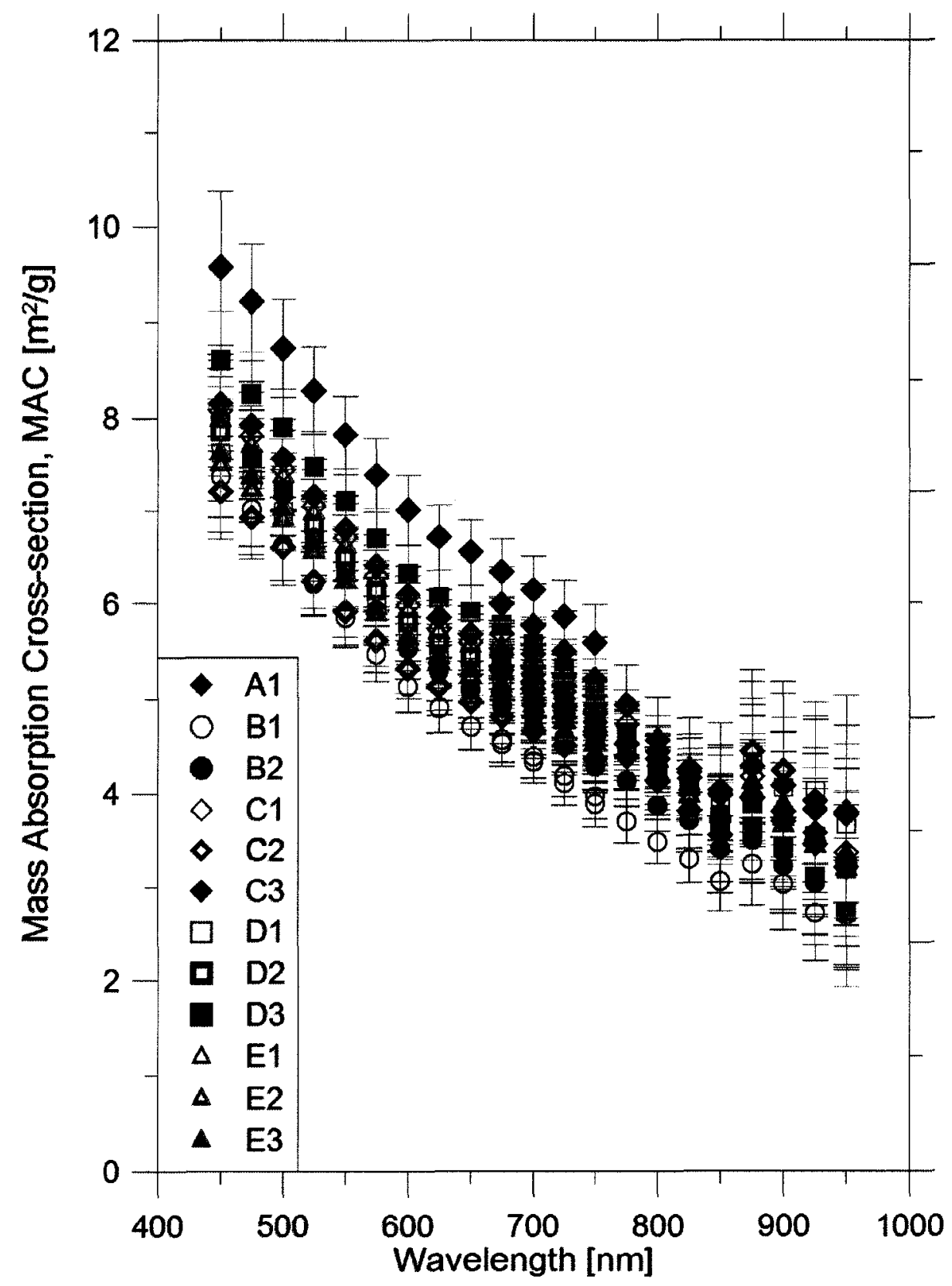

Figure 5.4: Mass absorption cross-sections for all conditions (refer to Table 3.1 on page 44 for further description of the burner conditions) 


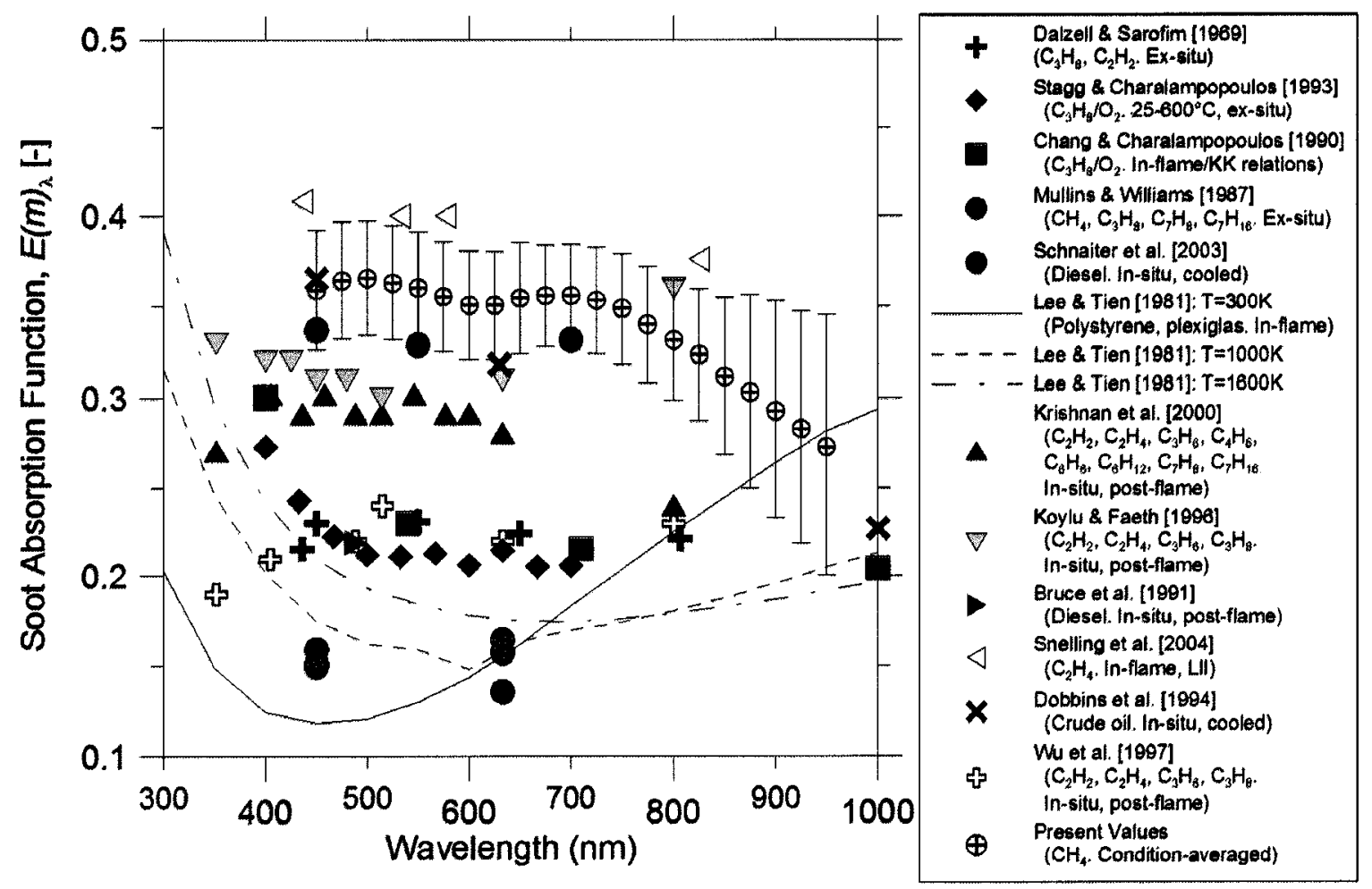

Figure 5.5: Condition-averaged $E(m)_{\lambda}$ data plotted alongside literature values. Samples for all ex-situ measurements were collected from within a flame.

able agreement, although they showed essentially the opposite trend with increasing wavelength as that found in the present work. This agreement further bolsters the generally accepted notion that soot optical properties are reasonably independent of fuel type, since the present study and the five listed above all use significantly different fuels (crude oil, various gaseous and liquid fuels, diesel, and ethylene). Nevertheless, an attempt was made to find correlation between the measured absorption properties and parameters pertaining to the fuel being burned. Appendix D contains a subset of these plots, using data from the studies shown in Figure 5.5.

One common assumption made is that the refractive index (and hence $E(m)$ ) is constant across the visible wavelengths. The present results show that this approximation is reasonable, although a small but consistent dip at around $600 \mathrm{~nm}$ was 
common to all measurements. However, the magnitude of this dip is small compared to the measurement uncertainty.

Many studies have reported values for the MAC, which were reviewed and presented by Bond and Bergstrom [2006]. After reviewing dozens of previous studies, they concluded that the value of $\sigma_{a}=10 \mathrm{~m}^{2} / \mathrm{g}$, commonly used in the field of atmospheric modelling [e.g. Seinfeld and Pandis, 1998] was erroneous. Instead, they suggested an alternative value of $\sigma_{a}=7.5 \pm 0.6 \mathrm{~m}^{2} / \mathrm{g}^{1}$ at a wavelength of $550 \mathrm{~nm}$, which they found as the average value of results in the literature for freshly-generated combustion aerosol. They considered 17 studies, and adjusted the values as required to account for both the filtration medium and for the wavelength of light used in the measurements. For the latter it was assumed that the refractive index of soot is constant across the visible range, resulting in an inverse relationship between MAC and wavelength. The present results support this assumption, and their recommended value is consistent (within uncertainty limits) with the present findings of $6.5 \pm 0.5 \mathrm{~m}^{2} / \mathrm{g}$ at that wavelength. Figure 5.6 shows a comparison between the present results and the literature values (original and adjusted) reviewed by Bond and Bergstrom [2006].

\subsection{Implications}

The choice of $E(m)_{\lambda}$ values have significant impact on the results of optical diagnostics. LII in particular is sensitive not only to the absolute value of the absorption function, but also the spectral variation or relative $E(m)$. It is often assumed that $E(m)$ is constant over the visible wavelengths [e.g. Michelsen, 2003, Snelling et al., 2005], with common values being $E(m)=0.26$ [Snelling et al., 2005], derived from the popular $m=1.57-0.56 \imath$ [e.g. Smyth and Shaddix, 1996], as well as $E(m)=0.4$ as

\footnotetext{
${ }^{1}$ The uncertainty has been converted to $95 \%$ confidence limits for comparison. The original uncertainty figure given in the paper was $1.2 \mathrm{~m}^{2} / \mathrm{g}$, corresponding to a single standard deviation.
} 


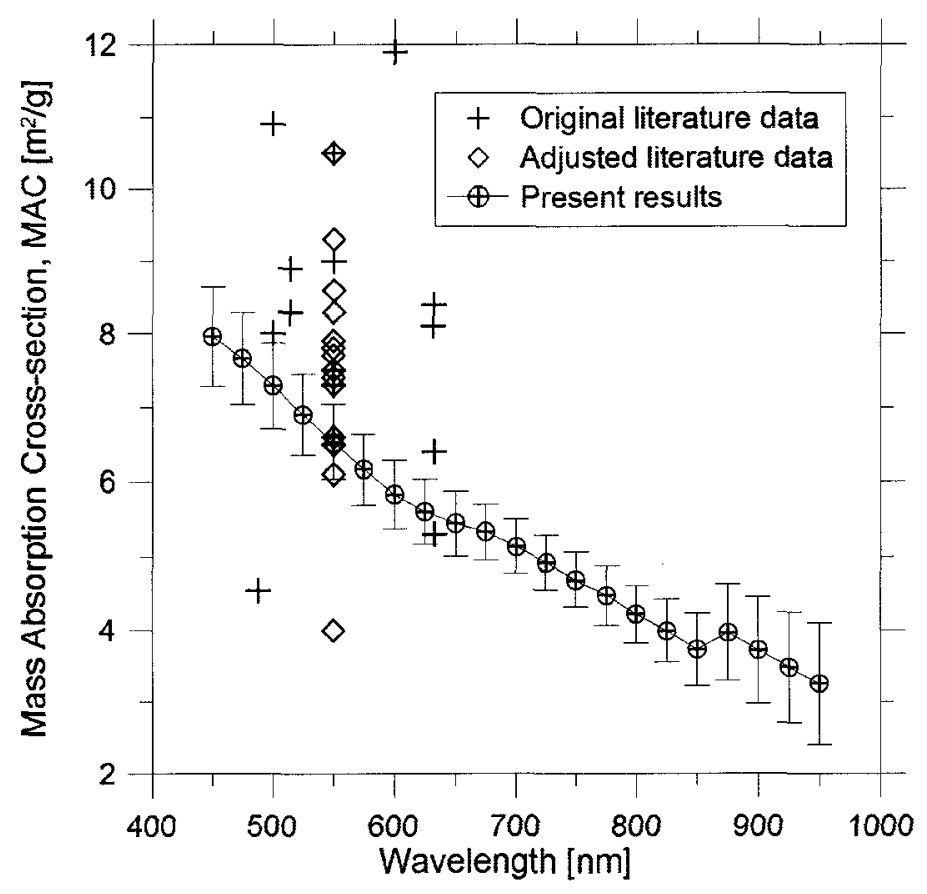

Figure 5.6: Comparison of present MAC results to the literature values reviewed and adjusted by Bond and Bergstrom [2006]

found by Snelling et al. [2004] and currently in use at the NRC. The peak soot volume fraction found using these values, as well as the current results (both condition-specific and averaged) are compared to those obtained by gravimetric analysis in Figure 5.7. Surprisingly, it is the commonly-used $E(m)=0.26$ that gives the best agreement in most cases, followed by the present results. The left-most data point and the two right-most data points are exceptions to the general linear trend seen between the gravimetric soot volume fraction and that found by LII. The left-most point corresponds to condition $\mathrm{C} 3$, which was found to be an outlier throughout this work. The two right-most data points correspond to the underventilated "B"-series, showing that these conditions are not well approximated by any of the constant or averaged values. This is perhaps an indication that flame conditions do have an effect on resulting optical properties. What is obvious, however, is that the fluence dependence of LII results for cooled soot is not simply a case of shifted optical properties; or 


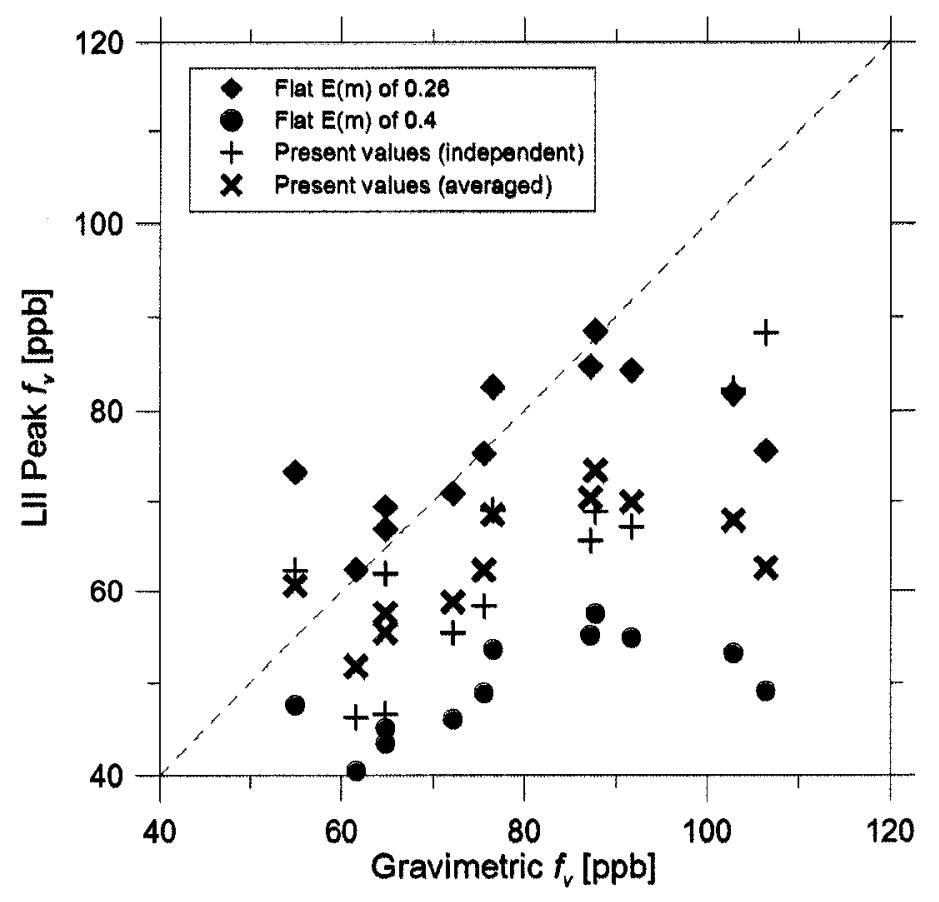

Figure 5.7: Comparison of peak soot volume fractions found by LII and gravimetric analysis

at least there is some significant difference between effective properties in extinction measurements and those in LII.

These results also have potential implications outside the combustion community. In the field of climate modelling, optical properties of soot have a critical impact on climate forcing models used in predicting global climate change. Soot is the primary absorber of visible solar radiation in the atmosphere, and its radiative forcing effect on the global energy budget is significant. Indeed, the magnitude of its forcing effect is estimated to be $16-28 \%$ of the widely-known greenhouse effect of $\mathrm{CO}_{2}$ [IPCC, 2007], with estimates as high as 55\% [Ramanathan and Carmichael, 2008], and greater than the contributions of other greenhouse gases such as methane, CFCs, $\mathrm{N}_{2} \mathrm{O}$, or tropospheric ozone [Ramanathan and Carmichael, 2008]. Chylek and Wong [1995] put forward a simple relationship to calculate the direct radiative forcing of an opticallythin aerosol layer, modified slightly by Bond and Bergstrom [2006] to obtain the 
following "simple forcing efficiency" (SFE) relation:

$$
\mathrm{SFE}=\frac{S_{0}}{4} \tau_{a t m}^{2}\left(1-F_{c}\right)\left[2\left(1-a_{s}\right)^{2} \beta \cdot \mathrm{MSC}-4 a_{s} \cdot \mathrm{MAC}\right]
$$

where $S_{0}$ is the solar irradiance, $\tau_{a t m}$ is the atmospheric transmission, $F_{c}$ is the cloud fraction, $a$ is the surface albedo (ratio of scatter to extinction), $\beta$ is the backscatter fraction, and MSC is the mass-specific scattering cross-section. SFE has units of watts per gram. If the assumption that the ratio of scatter to absorption (i.e., MSC/MAC or $\rho_{s a}$ ) is constant, then the SFE is simply directly proportional to the value of MAC. Thus, the present results return a SFE $35 \%$ lower than using the traditional MAC of $10 \mathrm{~m}^{2} / \mathrm{g}$, and $13 \%$ lower than the average value reported by Bond and Bergstrom [2006]. The effects of such differences could be significant considering the important contribution of radiative forcing by atmospheric soot on the global energy budget. 


\section{Chapter 6}

\section{Conclusions and Recommendations}

\subsection{Conclusions}

The objectives of this work were to measure the optical properties of soot from a methane flame that had been allowed to age and cool in the exhaust stream, to better reflect real-world conditions such as tailpipe or smokestack measurements. An inverted co-flow diffusion flame burner was designed as a repeatable source of soot for analysis, and several burner conditions were identified for study. The parameters varied among conditions were dilution rate, dilution temperature, and dilution gas. For the same flame condition, three dilution rates, each at three dilution temperatures, were identified, as well as one analogous condition with nitrogen as the diluent rather than air. In addition, two conditions were identified with a different, underventilated flame condition, one with air as the diluent and the other with nitrogen; in this latter case, there is no excess oxygen in the system, so possible oxidation effects could be isolated.

Soot absorption properties were measured by means of spectrally-resolved optical extinction measurements, with soot volume fraction measured by gravimetric analysis 
and a scattering correction based on RDG-PFA theory and soot morphology measured by electron microscopy. A detailed uncertainty analysis was performed to determine the accuracy and precision of the results.

The resulting values of the soot absorption function, $E(m)_{\lambda}$, displayed a distinct correlation with wavelength. This spectral variation $(\tilde{E}(m))$ was remarkably consistent in the visible range for wide variations in the cooling and dilution conditions in the exhaust. Some variation in $\tilde{E}(m)$ was noted for tests with underventilated flames, suggesting that flame condition could have an effect on the resulting optical properties of soot, although the differences were only slightly greater that calculated uncertainty limits.

Comparatively little variation was found in $E(m)$ at fixed wavelength for differences in dilution rate, dilution temperature, and dilution gas. No correlations were found between any of these properties and the physical soot properties, varied burner parameters, or other derived parameters.

The present results are in good agreement with several other studies found in the literature. In particular, the $E(m)_{\lambda}$ results of Dobbins et al. [1994] and Schnaiter et al. [2003] show similar magnitudes, and results of Krishnan et al. [2000] and Snelling et al. [2004] compare favourably in terms of $\tilde{E}(m)$. This agreement supports the notion that fuel type has little effect on optical properties, as these four studies all use different, widely-varied fuels; both liquid and gaseous, from crude oil to researchgrade methane. Thus, these results are encouraging for the combustion diagnostics community, as they show that cooled soot from a methane flame is comparable to other measurements made for cooled or aged soot [Dobbins et al., 1994, Schnaiter et al., 2003], for soot measured shortly post-flame [Krishnan et al., 2000], and for in-flame soot [Snelling et al., 2004].

Data from laser induced incandescence (LII) measurements were analyzed using 
$E(m)$ data derived in the present work as well as from other sources and compared with gravimetric measurements. Results suggest that variations in optical properties between in-flame and cooled soot are not likely responsible for the fluence dependence seen in LII measurements of cooled soot that does not exist for measurements of inflame soot.

In addition to the soot absorption function, results were re-cast in the form of dimensionless extinction constant and mass-specific absorption cross-section (MAC) in an attempt to find trends there as well. This analysis supported the common assumption of constant refractive index over the visible range, which should be encouraging for the climate modelling community. Calculated values were in agreement with the recommended MAC value given by Bond and Bergstrom [2006] for freshly-generated combustion aerosol, further strengthening their recommendation.

\subsection{Recommendations}

A significant amount of uncertainty was introduced in the extinction measurements because of the relatively weak signal; the rapid increase in uncertainty at the higher wavelengths indicated that the sensitivity limits of the spec-LOSA diagnostic were being approached. The results of this study also strengthen the idea that fuel type does not have a strong influence over the resulting optical properties of soot. It is therefore recommended for future workers to choose a fuel with a higher sooting propensity in order to increase the soot concentration, and hence the signal-to-noise ratio of the extinction measurement.

The image analysis of SEM microphotographs was also problematic. Uncertainties were introduced by coating the sample with a conductive material, which could be avoided through use of a Field Emission Gun SEM (FEG-SEM) or an Environmen- 
tal SEM (ESEM), which do not require such a coating. Further uncertainties were introduced in the image processing procedure; separating soot aggregates from the background filter material was difficult, requiring filtration and some corresponding losses in accuracy and spatial resolution. It is therefore recommended that an alternative method of obtaining a scattering correction, such as direct measurement of scattered light, be implemented.

A small difference in the relative $E(m)$ was seen between the two flame conditions tested. Although no physical differences in the soot were apparent in the SEM or TEM data, this does suggest that flame condition could have some effect on the resulting properties. It is recommended that this effect be investigated further in future work. 


\section{References}

T. Acharya and A.K. Ray. Image Processing: Principles and Applications. Wiley, 2005.

J. Appel, H. Bockhorn, and M. Frenklach. Kinetic modeling of soot formation with detailed chemistry and physics: Laminar premixed flames of $\mathrm{C}_{2}$ hydrocarbons. Combustion and Flame, 121(1-2):122-136, 2000.

ASME. Part 1: Measurement Uncertainty, Instruments and Apparatus. ANSI/ASME PTC 19.1, 1985.

I. Ayranci, R. Vaillon, N. Selcuk, F. Andre, and D. Escudie. Determination of soot temperature, volume fraction and refractive index from flame emission spectrometry. Journal of Quantitative Spectroscopy and Radiative Transfer, 104(2):266-276, March 2007.

H. Bockhorn, editor. Soot Formation in Combustion - Mechanisms and Models. Springer-Verlag, 1994.

H. Bockhorn, H. Geitlinger, B. Jungfleisch, Th. Lehre, A. Schön, Th. Streibel, and R. Suntz. Progress in characterization of soot formation by optical methods. Physical chemistry, chemical physics, 4(15):3780-3793, 2002. 
C.F. Bohren and D.R. Huffman. Absorption and Scattering of Light by Small Particles. Wiley-Interscience, 1983.

T.C. Bond and R.W. Bergstrom. Light absorption by carbonaceous particles: An investigative review. Aerosol Science and Technology, 40:27-67, 2006.

A.M. Brasil, T.L. Farias, and M.G. Carvalho. A recipe for image characterization of fractal-like aggregates. Journal of Aerosol Science, 30(10):1379-1389, 1999.

C.W. Bruce, T.F. Stromberg, K.P. Gurton, and J.B. Mozer. Trans-spectral absorption and scattering of electromagnetic radiation by diesel soot. Applied Optics, 30:1537$1546,1991$.

CEPA. Canadian Environmental Protection Act (CEPA). c.33, 1999.

H. Chang and T.T. Charalampopoulos. Determination of the wavelength dependence of refractive indices of flame soot. Proceedings of the Royal Society: Mathematical and Physical Sciences, 430(1880):577-591, September 1990.

M.Y. Choi, A. Hamins, G.W. Mulholland, and T. Kashiwagi. Simultanous optical measurement of soot volume fraction and temperature in premixed flames. Combustion and Flame, 99:174-186, 1994.

M.Y. Choi, G.W. Mulholland, A. Hamins, and T. Kashiwagi. Comparisons of the soot volume fraction using gravimetric and light extinction techniques. Combustion and Flame, 102:161-169, 1995.

P. Chylek and J. Wong. Effect of absorbing aerosols on global radiation budget. Geophysical Research Letters, 22(8):929-931, 1995.

A. D'Alessio, A. Di Lorenzo, F. Beretta, and C. Venitozzi. Optical and chemical investigations on fuel-rich methane-oxygen premixed flames at atmospheric pressure. In 
Fourteenth Symposium (International) on Combustion, pages 941-953, Pittsburgh, 1973. The Combustion Institute.

W.H. Dalzell and A.F. Sarofim. Optical constants of soot and their application to heat-flux calculations. Journal of Heat Transfer, 91:100-104, 1969.

W.H. Dalzell, G.C. Williams, and H.C. Hottel. A light-scattering method for soot concentration measurements. Combustion and Flame, 14(2):161-169, April 1970.

C.J. Dasch. One-dimensional tomography: a comparison of Abel, onion-peeling, and filtered backprojection methods. Applied Optics, 31(8):1146-1152, 1992.

J.L. Devore. Probability and Statistics for Engineering and the Sciences. Brooks/Cole, sixth edition, 2004.

R.A. Dobbins and C.M. Megaridis. Morphology of flame-generated soot as determined by thermophoretic sampling. Langmuir, 3:254-259, 1987.

R.A. Dobbins and C.M. Megaridis. Absorption and scattering of light by polydisperse aggregates. Applied Optics, 30(33):4747-4754, 1991.

R.A. Dobbins, G.W. Mulholland, and N.P. Bryner. Comparison of a fractal smoke optics model with light extinction measurements. Atmospheric Environment, 28 $(5): 889-897,1994$.

B.T. Draine and P.J. Flateau. Discrete-dipole approximation for scattering calculations. Journal of the Optical Society of America, 11(4):1491-1499, 1994.

S. Duhr and D. Braun. Why molecules move along a temperature gradient. Proceedings of the National Academy of Sciences of the USA, 103(52):19678-19682, December 2006. 
C.D. Elvidge, K.E. Baugh, B.T. Tuttle, A.T. Howard, D.W. Pack, C. Milesi, and E.H. Erwin. A twelve year record of national and global gas flaring volumes estimated using satellite data. Final report to the World Bank, NOAA, May 2007.

U.S. E.P.A. Method 9 - Visual determination of the opacity of emissions from stationary sources. NSPS Test Method EMTIC TM-009, United States Environmental Protection Agency, Emission Measurement Branch, October 1990.

ERCB. Upstream petroleum industry flaring and venting report: Industry performance for year ending December 31, 2007. Technical Report ST60B-2008, Energy Resources Conservation Board, June 2008.

T. L. Farias, Ü.Ö. Köylü, and M. G. Carvalho. Range of validity of the RayleighDebye-Gans theory for optics of fractal aggregates. Applied Optics, 35(33):65606567, November 1996.

A.V. Filippov, M. Zurita, and D.E. Rosner. Fractal-like aggregates: Relation between morphology and physical properties. Journal of Colloid and Interface Science, 229 (1):261-273, 2000 .

S.K. Friedlander and C.S. Wang. The self-preserving particle size distribution for coagulation by Brownian motion. Journal of Colloid and Interface Science, 22(2): 126-132, August 1966.

E. Hecht. Optics. Addison Wesley, fourth edition, 2002.

A.J. Hurd and W.L. Flower. In situ growth and structure of fractal silica aggregates in a flame. Journal of Colloid and Interface Science, 122(1):178-192, March 1988.

IEA. Key world energy statistics. Technical report, International Energy Agency, 2006. 
IPCC. Climate Change 2007 - The Physical Science Basis. Contribution of Working Group I to the Fourth Assessment Report of the IPCC. Technical report, Intergovernmental Panel on Climate Change, 2007.

M.F. Iskander, H.Y. Chen, and J.E. Penner. Optical scattering and absorption by branched chains of aerosols. Applied Optics, 28(15):3083-3091, 1989.

M.R. Johnson, J.L. Spangelo, and L.W. Kostiuk. A characterization of solution gas flaring in Alberta. Journal of the Air and Waste Management Association, 51: 1167-1177, 2001.

Ü. Ö. Köylü and G. M. Faeth. Structure of overfire soot in buoyant turbulent diffusion flames at long residence times. Combustion and Flame, 89(2):140-156, May 1992.

Ü. Ö. Köylü and G.M. Faeth. Optical properties of overfire soot in buoyant turbulent diffusion flames at long residence times. Journal of Heat Transfer, 116(1):152-159, 1994a.

Ü. Ö. Köylü and G.M. Faeth. Optical properties of soot in buoyant laminar diffusion flames. Journal of Heat Transfer, 116:971-979, 1994b.

Ü. Ö. Köylü and G.M. Faeth. Spectral extinction coefficients of soot aggregates from turbulent diffusion flames. Journal of Heat Transfer, 118(2):415-421, 1996.

Ü. Ö. Köylü, G.M. Faeth, T.L. Farias, and M.G. Carvalho. Fractal and projected structure properties of soot aggregates. Combustion and Flame, 100:621-633, $1995 \mathrm{a}$.

Ü. Ö. Köylü, Y. Xing, and D.E. Rosner. Fractal morphology analysis of combustiongenerated aggregates using angular light scattering and electron microscope images. Langmuir, 11(12):4848-4854, 1995b. 
S.S. Krishnan, K.C. Lin, and G.M. Faeth. Optical properties in the visible of overfire soot in large buoyant turbulent diffusion flames. Journal of Heat Transfer, 122(3): $517-524,2000$.

J.C. Ku and K.-H. Shim. A comparison of solutions for light scattering and absorption by agglomerated or arbitrarily-shaped particles. Journal of Quantitative Spectroscopy and Radiative Transfer, 47(3):201-220, 1992.

S.C. Lee and C.L. Tien. Optical constants of soot in hydrocarbon flames. In Proceedings of the 18th International Symposium on Combustion, pages 1159-1166. The Combustion Institute, 1981.

M.Y. Lin, R. Klein, H.M. Lindsay, H.A. Weitz, R.C. Ball, and P. Meakin. The structure of fractal colloidal aggregates of finite extent. Journal of Colloid Interface Science, 137(1):263-280, 1990.

F. Liu and D.R. Snelling. Evaluation of the accuracy of the RDG approximation for the absorption and scattering properties of fractal aggregates of flame-generated soot. In $A I A A$ 40th Thermophysics Conference, pages AIAA 2008-4362, 2008.

Media Cybernetics. Image-Pro Plus Reference Guide for Windows. Media Cybernetics, Silver Spring, MD, 2002.

C.M. Megaridis and R.A. Dobbins. Morphological description of flame-generated materials. Combustion Science and Technology, 71:95-109, 1990.

H.A. Michelsen. Understanding and predicting the temporal response of laser-induced incandescence from carbonaceous particles. Journal of Chemical Physics, 118(15): 7012-7045, 2003.

M.F. Modest. Radiative Heat Transfer. Academic Press, second edition, 2003. 
I.D. Morrison and S. Ross. Colloidal Dispersions: Suspensions, Emulsions, and Foams. Wiley-Interscience, 2002.

R.D. Mountain and G.W. Mulholland. Light scattering from simulated smoke agglomerates. Langmuir, 4(6):1321-1326, November 1988.

J. Mullins and A. Williams. The optical properties of soot: A comparison between experimental and theoretical values. Fuel, 66(2):277-280, February 1987.

C. A. Pope III, R.T. Burnett, M.J. Thun, E.E. Calle, K. Ito, and G.D. Thurston. Lung cancer, cardiopulmonary mortality, and long-term exposure to fine particulate air pollution. Journal of the American Medical Association, 287:1132-1141, 2002.

V. Ramanathan and G. Carmichael. Global and regional climate changes due to black carbon. Nature Geoscience, 1:221-227, 2008.

D.M. Roesslor. Kramers-Kronig analysis of reflection data. British Journal of Applied Physics, 16(8):1119-1123, 1965.

P. Salembier. Morphological multiscale segmentation for image coding. Signal Processing, 38(3):359-386, 1994.

M. Schnaiter, H. Horvath, O. Möhler, K.-H. Naumann, H. Saathoff, and O.W. Schöck. UV-VIS-NIR spectral optical properties of soot and soot-containing aerosols. Journal of Aerosol Science, 34(10):1421-1444, October 2003.

J.H. Seinfeld and S.N. Pandis. Atmospheric Chemistry and Physics: From Air Pollution to Climate Change. Wiley-Interscience, 1998.

R. Siegell and J.R. Howell. Thermal Radiation and Heat Transfer. McGraw-Hill, second edition, 1981. 
G.J. Smallwood. Personal communication, 2007.

K.C. Smyth and C.R. Shaddix. The elusive history of $m=1.57-0.56 \imath$ for the refractive index of soot. Combustion and Flame, 107(3):314-320, November 1996.

D.R. Snelling. Personal communication, 2008.

D.R. Snelling, K.A. Thomson, G.J. Smallwood, and Ö.L. Gülder. Two-dimensional imaging of soot volume fraction in laminar diffusion flames. Applied Optics, 38(12): 2478-2485, April 1999.

D.R. Snelling, F. Liu, G.J. Smallwood, and Ö.L. Gülder. Determination of the soot absorption function and thermal accommodation coefficient using low-fluence LII in a laminar coflow ethylene diffusion flame. Combustion and Flame, 136(1-2): 180-190, January 2004.

D.R. Snelling, G.J. Smallwood, F. Liu, Ö.L. Gülder, and W.D. Bachalo. A calibrationindependent laser-induced incandescence technique for soot measurement by detecting absolute light intensity. Applied Optics, 44(31):6773-6785, 2005.

C.M. Sorensen. Light scattering by fractal aggregates: A review. Aerosol Science and Technology, 35(2):648-687, August 2001.

C.M. Sorensen, J. Cai, and N. Lu. Test of static structure factors for describing light scattering from fractal soot aggregates. Langmuir, 8(8):2064-2069, August 1992a.

C.M. Sorensen, J. Cai, and N. Lu. Light-scattering measurements of monomer size, monomers per aggregate, and fractal dimension for soot aggregates in flames. Applied Optics, 31(30):6547-6557, 1992b. 
B.J. Stagg and T.T. Charalampopoulos. Refractive indices of pyrolytic graphite, amorphous carbon, and flame soot in the temperature range $25^{\circ}$ to $600^{\circ} \mathrm{C}$. Combustion and Flame, 94(4):381-396, 1993.

C.B. Stipe, B.S. Higgins, D. Lucas, C.P. Koshland, and R.F. Sawyer. Inverted co-flow diffusion flame for producing soot. Review of Scientific Instruments, 76(2):023908, 2005 .

E. Taylor. Filter weighing procedure for 2007 and newer heavy duty diesel engines. Master's thesis, West Virginia University, 2006.

K.A. Thomson, M.R. Johnson, D.R. Snelling, and G.J. Smallwood. One-dimensional spectral line-of-sight attenuation for soot optical property measurement. In Proceedings of the Combustion Institute / Canadian Section Spring Technical Meeting, 2008, pages 23-28, May 2008a.

K.A. Thomson, M.R. Johnson, D.R. Snelling, and G.J. Smallwood. Diffuse-light twodimensional line-of-sight attenuation for soot concentration measurements. Applied Optics, 47(5):694-703, February 2008b.

C.-J. Tsai, C.-T. Chang, B.-H. Shih, S.G. Aggarwal, S.-N. Li, H.M. Chein, and T.-S. Shih. The effect of environmental conditions and electrical charge on the weighing accuracy of different filter materials. The Science of the Total Environment, 293: 201-206, 2002.

P. Van-Hulle, M.-E. Weill, M. Talbaut, and A. Coppalle. Comparison of numerical studies characterizing optical properties of soot aggregates for improved EXSCA measurements. Particle and Particle Systems Characterization, 19:47-57, 2002. 
R.L. Vander Wal and K.A. Jensen. Laser-induced incandescence: excitation intensity. Applied Optics, 37(9):1607-1616, 1998.

J.-S. Wu, S.S. Krishnan, and G.M. Faeth. Refractive indices at visible wavelengths of soot emitted from buoyant turbulent diffusion flames. Journal of Heat Transfer, 119(2):230-238, 1997.

J. Xi and B.-J. Zhong. Soot in diesel combustion systems. Chemical Engineering and Technology, 29(6):665-673, 2006.

J. Yon, C. Roze, T. Girasole, A. Coppale, and L. Mees. Extension of RDG-FA for scattering prediction of aggregates of soot taking into account interactions of large monomers. Particle and Particle Systems Characterization, 25(1):54-67, 2008.

A.T. Young. Rayleigh scattering. Physics Today, 35(1):42-48, 1982. 


\section{Appendix A}

\section{Burner Repeatability}

The inverted flame burner was selected for its steady, repeatable production of soot [Stipe et al., 2005]. The repeatability of the burner designed for the present work was verified using a scanning mobility particle sizer (SMPS) (TSI, Model 3936), which measures the particle size distribution in terms of electric mobility diameter. Note that due to the difficulty of converting the electric mobility diameter to a physical parameter such as radius of gyration, the SMPS data were considered to be qualitative only. Figure A.1 demonstrates the low variability found among repeated measurements of the same burner condition. 


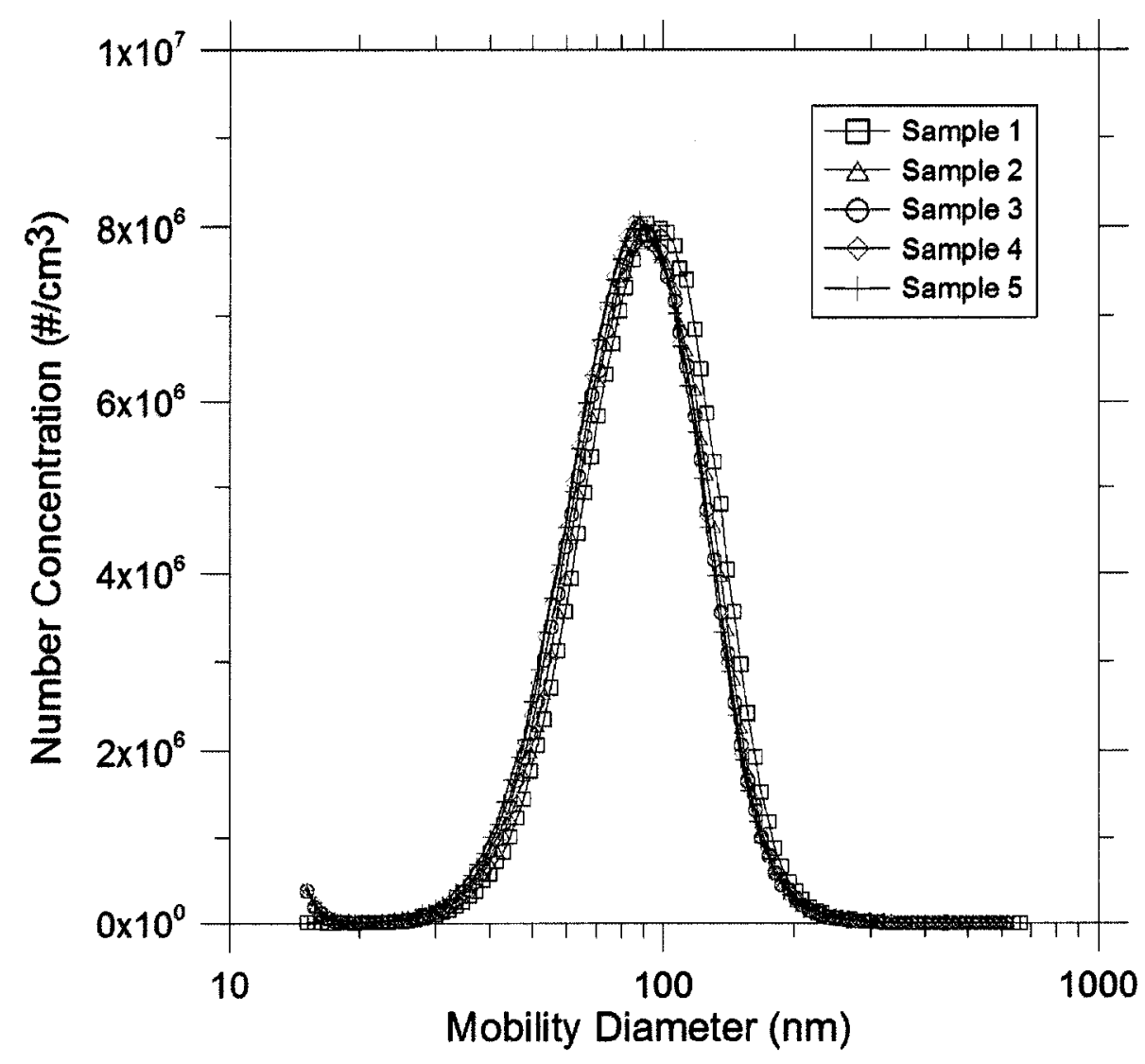

Figure A.1: Repeated SMPS measurements of a singe condition 


\section{Appendix B}

\section{Mathcad Analysis Files}

\section{B.1 Fitting Aggregate Size Distributions}

The following Mathcad sheet was used to fit statistical distributions to the aggregate size data from the SEM analysis described in Chapter 3. An outline of the steps taken in this sheet are as follows:

- Read in raw aggregate size data $(N)$

- Create normalized histogram of $N$ data with log-based bin widths

- Fit data to log-normal distribution with $95 \%$ confidence limits

- Fit data to self-preserving distribution with $95 \%$ confidence limits

- Output parameters to file for later use 


\title{
Analysis of $N$ values from SEM data
}

The analysis is derived from Dave's analysis of previous TEM work. The original file is:

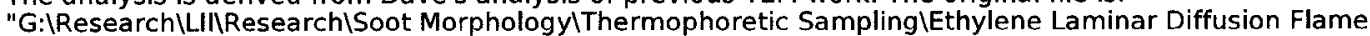
tests\Analysis of $\mathrm{N}$ values from TEM images.xmcdz"

Which condition are we analyzing? condition: $=" \mathrm{A1}$

Are we writing output files? (1 for yes) write bool $=0$

\section{Define file locations, bring in references.}

\author{
$\boxplus$ Reference:K:|LII|Templates(Useful_2001.mcd

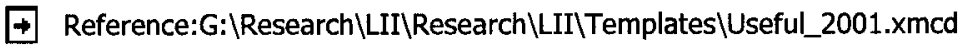 \\ $\pitchfork$ Reference:Z:|Research|LII \Templates|Useful_2001.xmcd

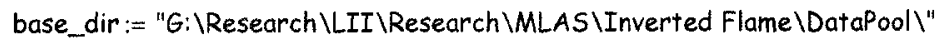 \\ input_file := concat(base_dir, condition, "-N.tXt")

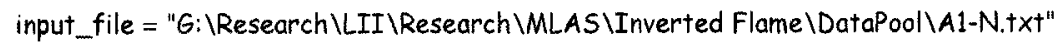

Read in data: N_data:=READPRN(input_file) $\langle\hat{\psi}$

\section{Normalized Histogram}

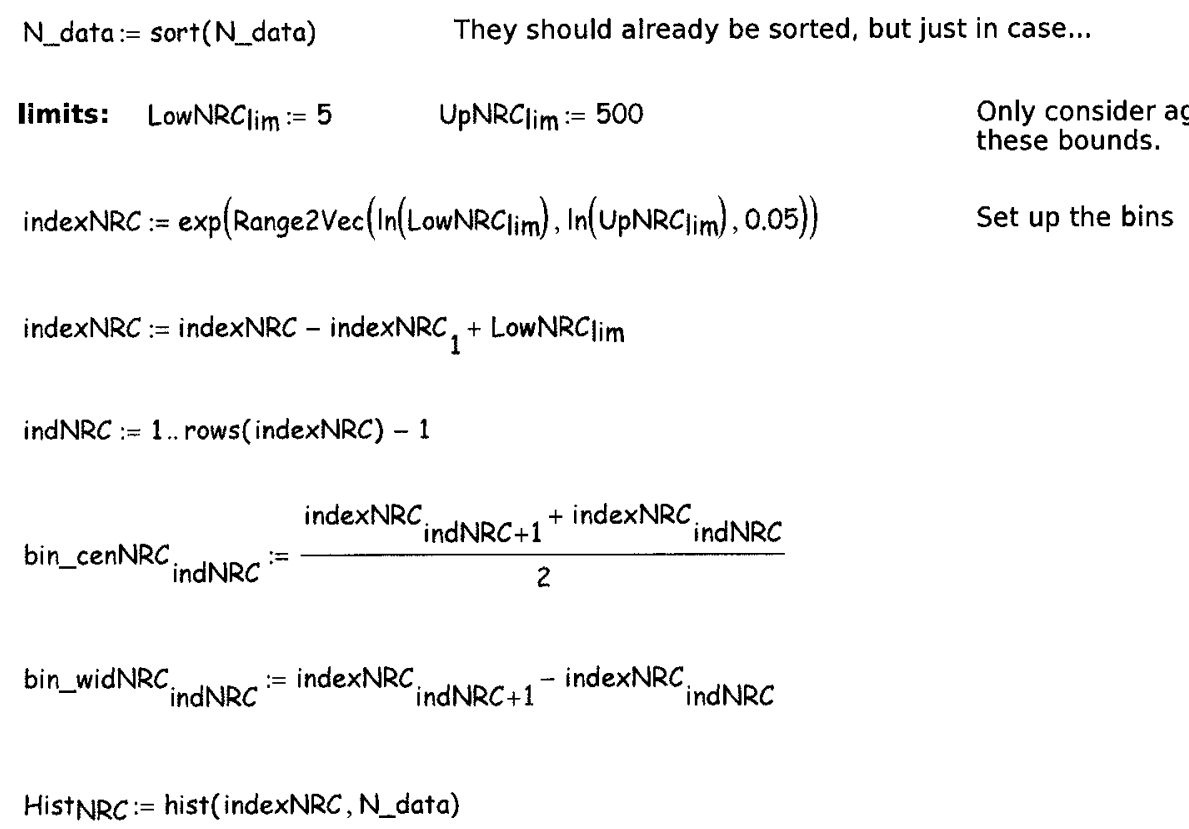


HistNRC: $=\frac{\text { HistNRC }}{\sum_{i=1}^{\text {rows }(\text { HistNRC) }} \text { HistNRC }_{i, 1}}$

Normalize distribution: $\quad \sum_{i=1}^{\operatorname{rows}\left(\text { HistNRC } \text { HistNRC }_{\mathrm{i}, 1}=1\right.}$

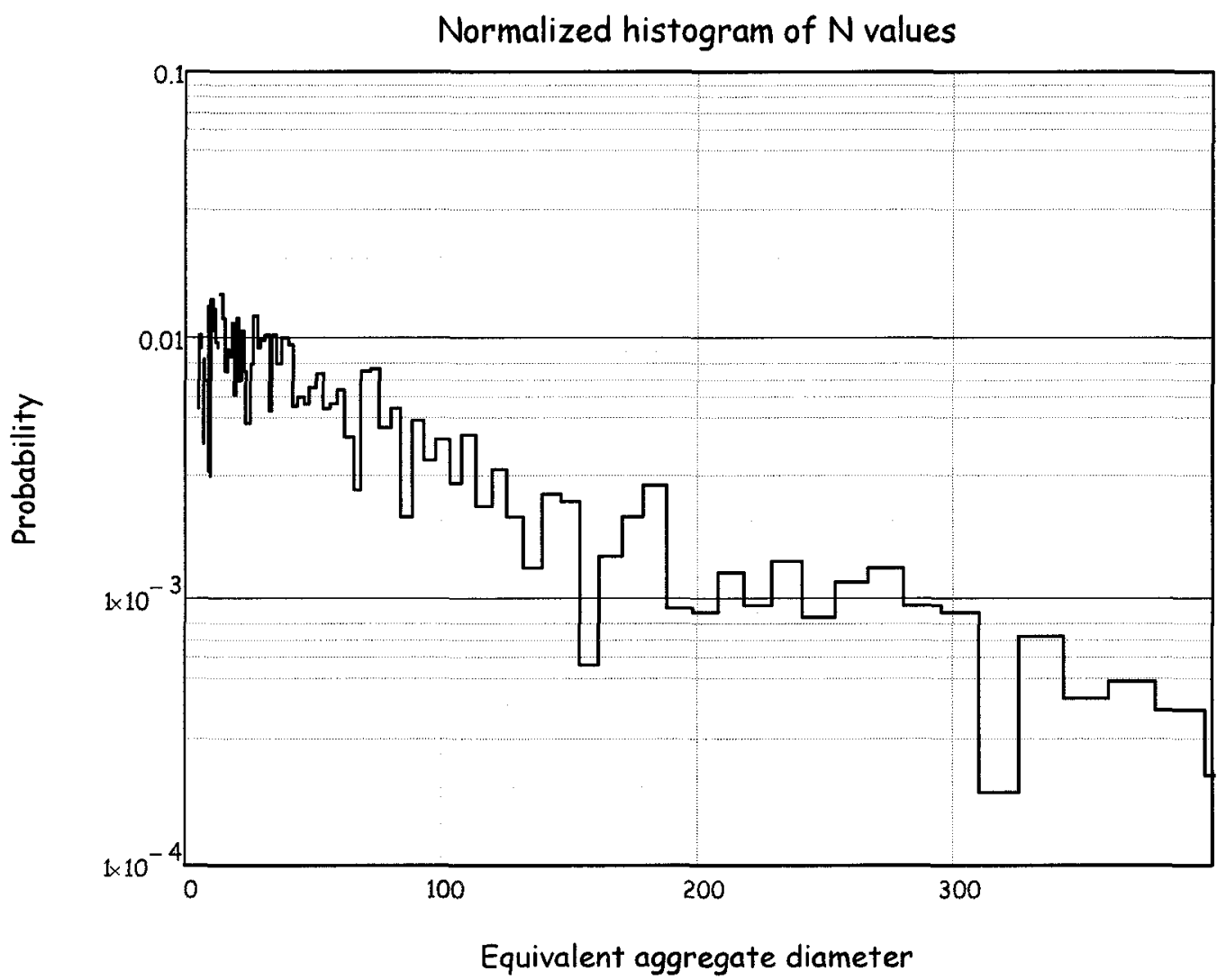

Fit data using LeastSquareFit function that returns confidence limits

Lognormal fit

General form of log-normal distribution:

$$
F_{-} \operatorname{lognorm}(N, u):=\frac{\exp \left[-\left[\frac{\ln (N)-\ln \left(u_{1}\right)}{\frac{1}{2} \cdot \ln \left(u_{2}\right)}\right)^{2}\right]}{N \cdot \ln \left(u_{2}\right) \cdot(2 \cdot \pi)^{5}}
$$


Fitting function (normazized within the limits above):

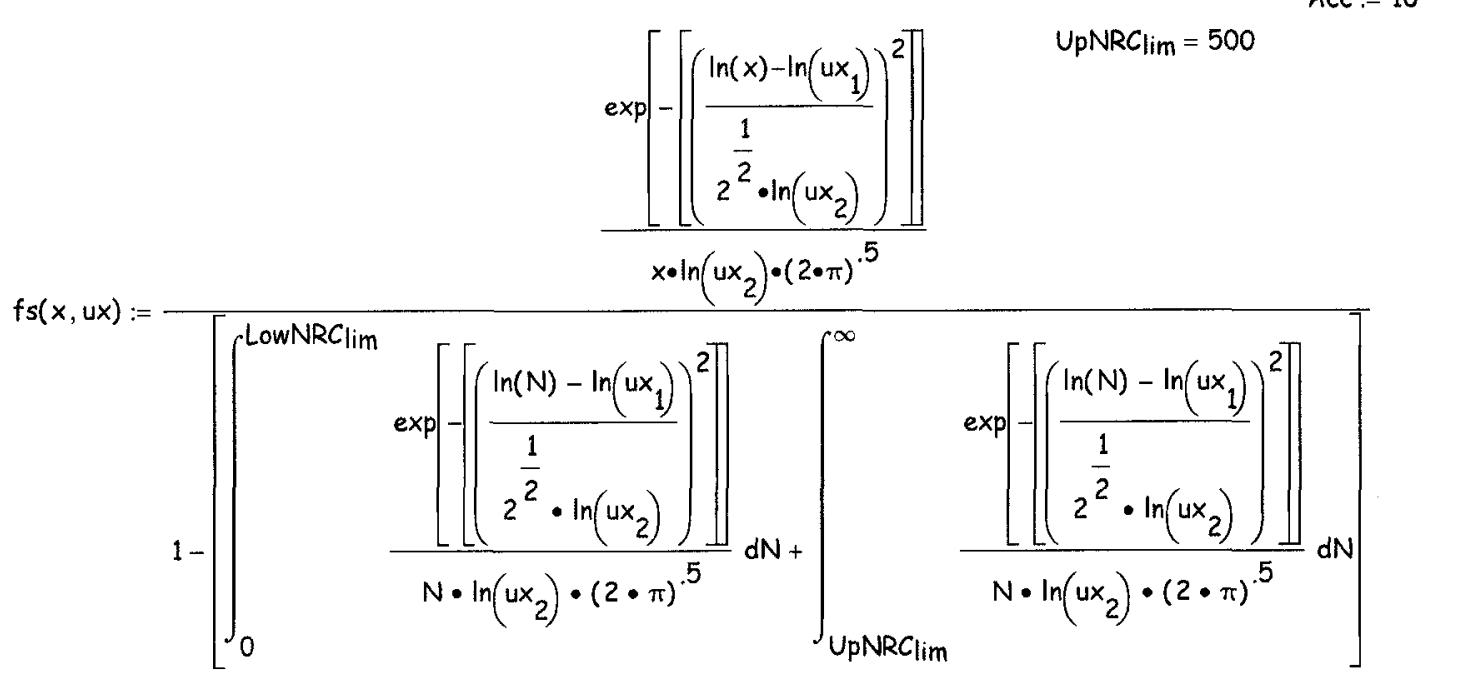

Guess values: $\quad$ initial_guess :=( $\left.\begin{array}{c}95 \\ 2.75\end{array}\right) \quad \frac{H_{i s t N R C_{i}}}{\operatorname{bin} \_w i d N R C_{i}} \cdot\left(1-f_{\ln }\right)-f \operatorname{logn}($ bin_cenNRC,$u)$

Fit the function:

$$
\text { ResLN := LeastSquaresFit }\left[\text { bin_cenNRC, }\left(\frac{\text { HistNRC }}{\text { bin_widNRC }}\right) \text {,fs, initial_guess, 0.95, Acc }\right]
$$

Inputs

The $x$ and $y$ data are the first two arguments, followed by the fit function, $f(x$,$) . Next is the guess vector guess for$ the parameter values, followed by the desired confidence limit on the parameters, 0.95 , or $95 \%$. Accv is an optional variable whose default is $10^{\wedge}-7$ the solution here is obtained with a value of $A c c=10^{\wedge}-12$

$$
\text { ir }:=1 . .3
$$

$$
\operatorname{Res} L N=\left(\begin{array}{ccc}
80.892 & 66.05 & 95.734 \\
3.392 & 2.939 & 3.846
\end{array}\right) \quad N_{\text {mean }}=N g \exp \left(0.5 \cdot \ln (\sigma 2 g)^{2}\right)
$$

Mean aggregate sizes are: $\quad(\operatorname{Res} L N)_{1, \mathrm{ir}} \exp \left[0.5 \cdot\left(\ln \left(\operatorname{Res} L N_{2}, \mathrm{ir}\right)\right)^{2}\right]=$

170.584

118.099

237.181 


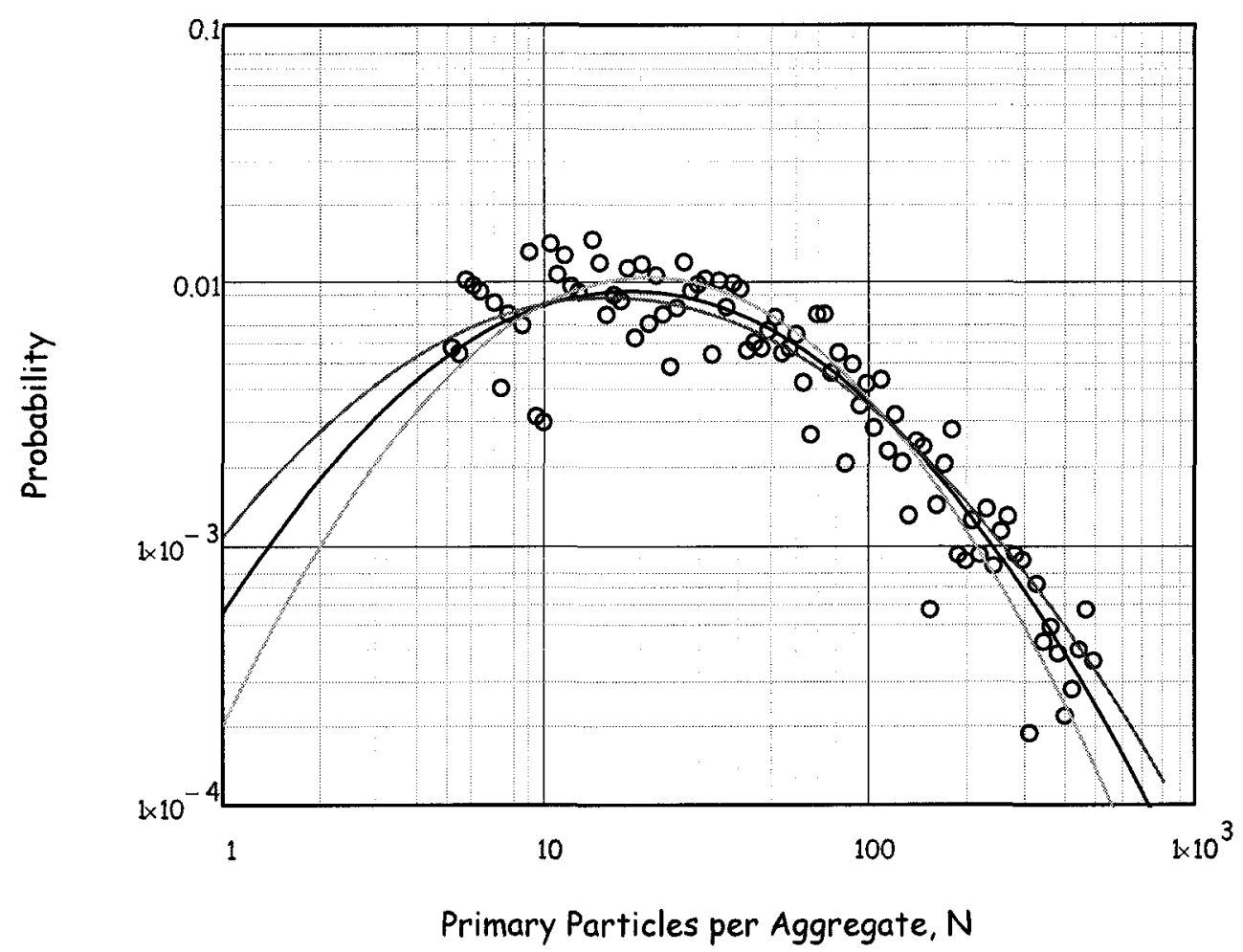

Ooo Data

Best fit

Lower $95 \%$ confidence limits

Upper $95 \%$ confidence limit

$i:=1 . .3$

$j:=1 . .3$

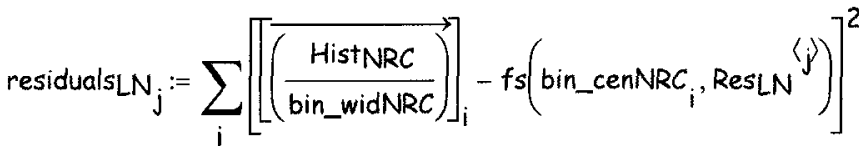

residuals $L N_{j}=$

$1.921 \cdot 10^{-5}$

$2.823 \cdot 10^{-5}$

$1.459 \cdot 10^{-5}$

Note concening function Res.

The solver uses sequential quadratic programming (SQP) to solve the resulting least-squares problem. By introducing additional variables, the original problem is transformed into a general equality constrained nonlineal programming problem, which is, in general, faster and more stable than some other methods.

The first column of output contains the values for the fitted parameters. The second column contains the left and the third column the right boundary for the confidence interval on the parameters. 


\section{Self preserving fit}

General form: $\quad n(N)=\frac{M_{1}}{s_{p}^{2}} \cdot \phi\left(\frac{N}{s_{p}}\right)=\frac{M_{1}}{s_{p}^{2}} \cdot\left[A \cdot\left(\frac{N}{s_{p}}\right)^{-\tau} \cdot \exp \left(-\alpha \cdot \frac{N}{s_{p}}\right)\right] \quad \begin{aligned} & \begin{array}{l}\text { where } A \text { and } \alpha \text { are } \\ \text { constants to be } \\ \text { determined }\end{array} \\ & \text { detants }\end{aligned}$

If we define $s_{p}$ as being the first moment i.e. $s_{p}=\frac{M_{1}}{M_{0}}$

and remembering we have a normalised distribution so that

Mo $=1$

the distribution takes the form:

$$
n(N)=\frac{1}{M_{1}} \cdot\left[\frac{(1-\tau)^{2-\tau}}{\Gamma(2-\tau)} \cdot\left(\frac{N}{M_{1}}\right)^{-\tau} \cdot \exp \left[\frac{-N \cdot(1-\tau)}{M_{1}}\right]\right]
$$

Fitting function

$$
\text { Acc }:=10^{-12}
$$

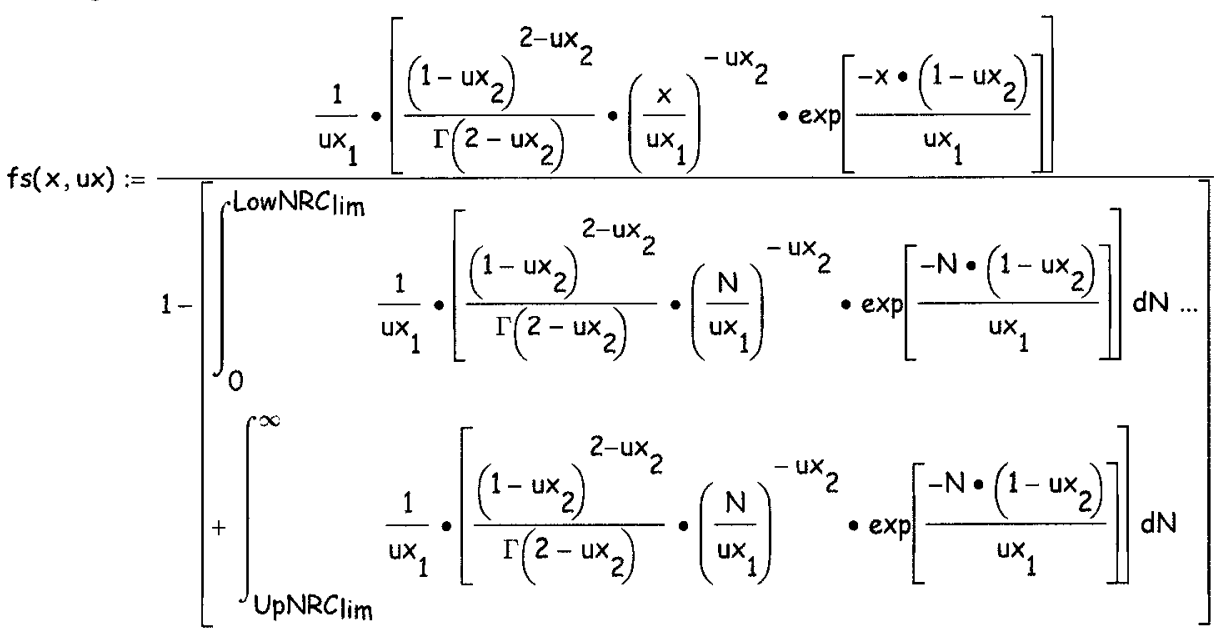

Guess values: $\quad$ initial_guess : $=\left(\begin{array}{l}75 \\ 0.4\end{array}\right)$

Fit the function:

$$
\text { ResSP:= LeastSquaresFit }\left[\text { bin_cenNRC, } \overrightarrow{\left(\frac{H i s t N R C}{\text { bin_widNRC }}\right)} \text {,fs, initial_guess, 0.95, Acc }\right] \text { si :=1..rows(bin_cenNRC) }
$$

Inputs

The $x$ and $y$ data are the first two arguments, followed by the fit function, $f(x$,$) . Next is the guess vector guess for$ the parameter values, followed by the desired confidence limit on the parameters, 0.95 , or $95 \%$. Accv is an optio variable whose default is $10^{\wedge}-7$ the solution here is obtained with a value of $A c c=10^{\wedge}-12$

$$
\text { Res SP }=\left(\begin{array}{ccc}
89.883 & 77.535 & 102.232 \\
-0.309 & -0.502 & -0.115
\end{array}\right) \quad i:=1 . .3
$$




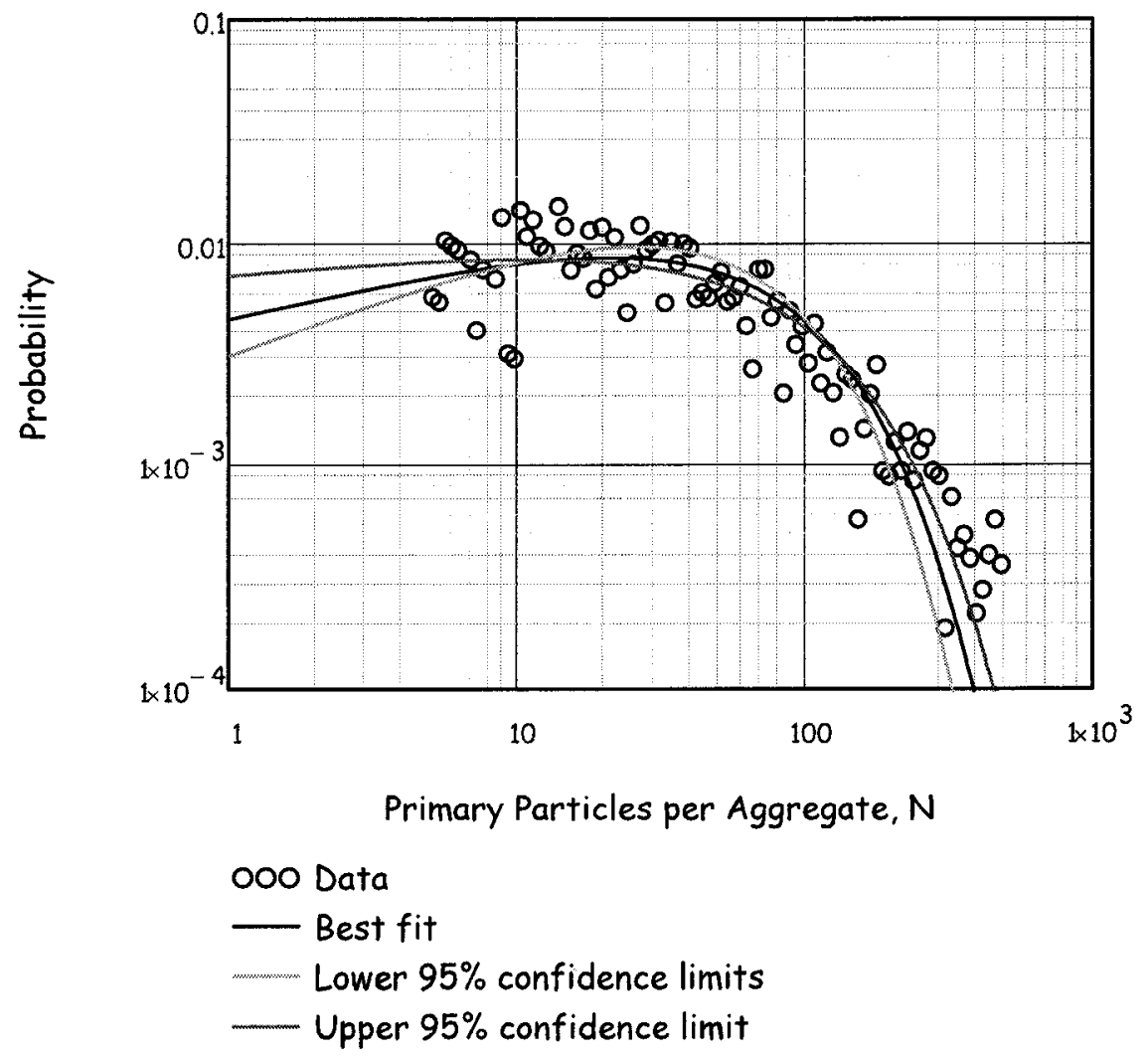

$$
\begin{aligned}
& \text { residuals } S P_{j}:=\sum_{i}\left[\left[\overrightarrow{\left(\frac{H i s t N R C}{\text { bin_widNRC }}\right)}\right]_{i}-f s\left(\text { bin_cenNRC } i, \operatorname{Res} S P^{\langle j}\right)\right]^{2} \quad \text { residuals } S P_{j}= \\
& 1.414 \cdot 10^{-5} \\
& 1.484 \cdot 10^{-5} \\
& 1.828 \cdot 10^{-5}
\end{aligned}
$$

\section{Output section:}

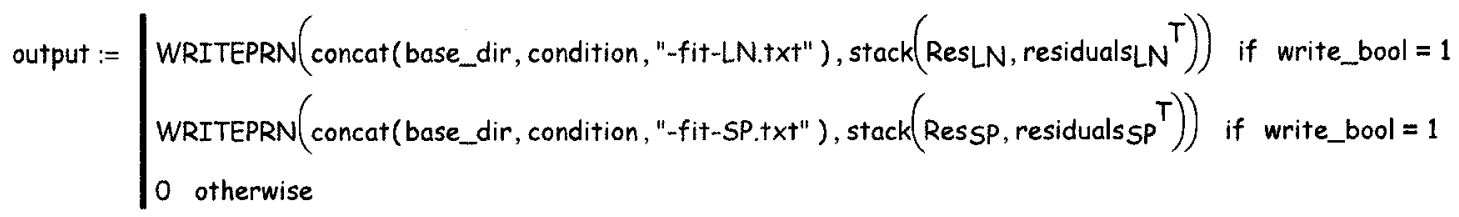




\section{B.2 Scatter-to-Absorption Ratio}

Two analysis files are included here. The first is a reference sheet, defining equations and functions to be used by the second sheet, which does the actual calculation.

\section{B.2.1 Reference Sheet}

Equations are defined in this sheet for use in calculating $\rho_{s a}$. This sheet is essentially the relevant subset of scattering equations developed into functional form by Dr. Snelling of the NRC. Note that several equations are much wider than a single page, and have been truncated by the software. This sheet is used by including it as a reference in other sheets, thereby giving access to the equations contained herein. 


\section{RDG/PFA scattering/absorption equations}

This is indended as a reference, other sheets do the actual calculations

This sheet is based off Dave Snelling's scattering worksheets. (Internal: See K:|LIIIExperiments\DavelScattering|Scattering Theory use me.mcd for equation development)

Define constants and distribution equations:

$$
n m:=10^{-9} \cdot m \quad N_{A}:=6.0221367 \cdot 10^{23} \cdot \text { mole }^{-1}
$$

Lognormal equation:

$$
\text { F2_lognorm }(N, N g, \sigma 2 g):=\frac{\exp \left[-\left[\left(\frac{\ln (N)-\ln (N g)}{2^{5} \cdot \ln (\sigma 2 g)}\right)^{2}\right]\right]}{N \cdot \ln (\sigma 2 g) \cdot(2 \cdot \pi)^{.5}}
$$

First moment (mean value of $N$ ) of lognormal distribution

$$
N_{\text {mean }}(N g, \sigma 2 g):=N g \exp \left(0.5 \cdot \ln (\sigma 2 g)^{2}\right)
$$

Self preserving distribution

$$
\operatorname{PSP}\left(N, M_{1}, \tau\right):=\frac{1}{M_{1}} \cdot\left[\frac{(1-\tau)^{2-\tau}}{\Gamma(2-\tau)} \cdot\left(\frac{N}{M_{1}}\right)^{-\tau} \cdot \exp \left[\frac{-N \cdot(1-\tau)}{M_{1}}\right]\right]
$$

where $M_{1}$ is the first moment or mean particle size

\section{Scattering and absorption equation integrated over lognormal distribution:}

Note that this is the total scattering coefficient for the normalized lognormal distribution and to get the total differential scattering per unit volume we must multiply the result by the number density of aggregates $\mathrm{N}_{a g}$ given by:

$$
N_{a g}(f v, N g, d p, \sigma 2 g)=\frac{f v \cdot 10^{-6}}{\frac{\pi \cdot d p^{3}}{6} \cdot\left(N g \exp \left(0.5 \cdot \ln (\sigma 2 g)^{2}\right)\right)}
$$


Using the confluent hypergeometric function (Sorensen, 1992, 2001)

Integrated aggregate cross section over lognormal distribution

$L N \_\sigma_{-} C H G_{v v}(\theta, D f, k f, \lambda, d p, N g, \sigma 2 g, F M):=\left(\frac{\pi^{4} \cdot d p^{6} \cdot F M}{4 \cdot \lambda^{4} \cdot s r}\right) \cdot\left[\int_{1}^{\infty} n^{2} \cdot F 2 \_l o g n o r m(n, N g, \sigma 2 g) \cdot\right.$ if $\left[\frac{4 \cdot \pi}{\lambda} \cdot \sin \left(\frac{e}{i}\right.\right.$

Calculate total scattering cross section for lognormal distribution of aggregates:

$$
\text { Scatteringtotal }=\int_{0}^{\pi}\left[\frac{d \sigma}{d \Omega} \cdot\left(\frac{1+\cos (\theta)^{2}}{2}\right) \cdot 2 \cdot \pi \cdot \sin (\theta)\right] d \theta=\frac{8}{3} \cdot \frac{d \sigma}{d \Omega} \cdot \pi
$$

see K:|LIIIExperiments|DavelScattering|Geometry_of_scattering.mcd

$\sigma A_{-} C F G_{s c a}(D f, k f, F M, \lambda, d p, N g, \sigma 2 g):=2 \cdot \pi \cdot \mathrm{nm}^{2} \cdot \int_{0.000}^{\pi} \frac{L N_{-} \sigma_{-} C H G_{v v}(\theta, D f, k f, \lambda, d p, N g, \sigma 2 g, F M)}{n^{2}} \cdot \frac{1+\cos (t}{2}$

Absorption cross section:

$\sigma A a b s(E M, \lambda, d p, N g, \sigma 2 g):=\pi^{2} \cdot \frac{d p^{3}}{\lambda} \cdot E M \cdot \int_{1}^{\infty} F 2 \_\operatorname{lognorm}(n, N g, \sigma 2 g) \cdot n d n$

Scatter-to-absorption ratio for lognormal distribution of aggregates:

Using above functions

$$
\rho S A_{-} C H G(D f, k f, E M, \lambda, d p, N g, \sigma 2 g, F M):=\frac{\sigma A_{\_} C F G_{s c a}(D f, k f, F M, \lambda, d p, N g, \sigma 2 g)}{\sigma A_{a b s}(E M, \lambda, d p, N g, \sigma 2 g)}
$$

An examination of the equations above shows that $\sigma A_{-} C F G_{s c a}$ is linearly proportional to $F(m)$ and that $\sigma A_{\text {abs }}$ is linearly proportional to $E(m)$ so that we can recast the equation for the scattering to absorption ratio in the form

$$
\rho S A 2 \_C H G(D f, k f, \lambda, d p, N g, \sigma 2 g, F M E M):=\frac{\sigma A \_C F G_{\text {sca }}(D f, k f, F M E M, \lambda, d p, N g, \sigma 2 g)}{\sigma A \text { abs }(1, \lambda, d p, N g, \sigma 2 g)}
$$

\section{Scattering and absorption equations integrated over self preserving distribution:}


Using the confluent hypergeometric function (Sorensen, 1992, 2001)

Integrated aggregate cross section over self preserving distribution

$S P_{-} \sigma_{-} C H G_{w}\left(\theta, D f, k f, \lambda, d p, M_{1}, \tau, F M\right):=\left(\frac{\pi^{4} \cdot d p^{6} \cdot F M}{4 \cdot \lambda^{4} \cdot s r}\right) \cdot\left[\int_{0}^{\infty} n^{2} \cdot P_{S P}\left(n, M_{1}, \tau\right) \cdot\right.$ if $\left[\frac{\left[\frac{4 \cdot \pi}{\lambda} \cdot \sin \left(\frac{\theta}{2}\right) \cdot\left(\frac{n}{k f}\right)^{\bar{C}}\right.}{D f}\right.$

Total scattering

$\sigma A_{-} C F G_{-} \operatorname{scaSP}\left(D f, \mathrm{kf}, \mathrm{FM}, \lambda, \mathrm{dp}, M_{1}, \tau\right):=2 \cdot \pi \cdot \mathrm{nm}^{2} \cdot \int_{0.000}^{\pi} \frac{S P_{-} \sigma_{-} C H G_{\mathrm{vv}}\left(\theta, D f, \mathrm{kf}, \lambda, \mathrm{dp}, M_{1}, \tau, F M\right)}{\mathrm{nm}^{2}} \cdot \frac{1+\cos (\theta)^{i}}{2}$

Absorption cross section:

$$
\sigma A_{-} \text {abs SP }\left(E M, \lambda, d p, M_{1}, \tau\right)=\pi^{2} \cdot \frac{d p^{3}}{\lambda} \cdot E M \cdot \int_{0}^{\infty} P S P\left(N, M_{1}, \tau\right) \cdot n d n
$$

The integral is the first moment or mean aggregate size and is given by $M_{1}$, so that:

$$
\sigma A \_a b s S P\left(E M, \lambda, d p, M_{1}\right):=\pi^{2} \cdot \frac{d p^{3}}{\lambda} \cdot E M \cdot M_{1}
$$

\section{Scattering to absorption ratio for self preserving distribution}

Using above functions

$$
\rho S A \_C H G S P\left(D f, k f, E M, \lambda, d p, M_{1}, \tau, F M\right):=\frac{\sigma A \_C F G \_s c a s P\left(D f, k f, F M, \lambda, d p, M_{1}, \tau\right)}{\sigma A_{-} \text {abs SP }\left(E M, \lambda, d p, M_{1}\right)}
$$

As per above, this equation can be re-cast using the ratio of $F(m) / E(m)$, as follows:

$$
\rho S A 2 \_C H G S P\left(D f, k f, \lambda, d p, M_{1}, \tau, F M E M\right):=\frac{\sigma A \_C F G \_s c a S P\left(D f, k f, F M E M, \lambda, d p, M_{1}, \tau\right)}{\sigma A_{-} a b s S p\left(1, \lambda, d p, M_{1}\right)}
$$




\section{B.2.2 Calculation of $\rho_{s a, \lambda}$}

This sheet was used to calculate the scatter-to-absorption ratio over the range of 450 $950 \mathrm{~nm}$, in $25-\mathrm{nm}$ increments. Inputs to this sheet are the fractal parameters $\left(D_{f}, k_{g}\right)$ and morphological information ( $d_{p}$, distribution parameters) found through ex-situ diagnostics as discussed in Chapter 3. Outputs are tables of $\rho_{s a, \lambda}$ values at $25-\mathrm{nm}$ increments, along with fractional uncertainty limits, for both the log-normal and the self-preserving distributions. Uncertainty propagation was performed by parametric analysis, whereby the contributing parameters were varied to their bias limits and the resulting change in calculated values was observed. For simplicity, uncertainty was assumed to be symmetric about the mean value, which implies that $\rho_{s a}$ varies linearly with each input parameter. While this is not strictly the case, sample calculations show that the differences are small. 


\section{$\rho_{S A}$ from RDG-PFA}

This sheet is based on Dr. Snelling's RDG/PFA scattering worksheets.

See K:|LIIIExperiments|DavelScattering|Scattering Theory use me.mcd for equation development.

Equations and whatnot imported from the following reference:

$\oplus$ Reference:K:\MLAS Inverted Flame\MathCAD\Reference - RDG_PFA equations.xmcd

Inputs are fractal info, stat fits of SEM data and $d_{p}$. Output is a table of $\rho_{\text {SA }}$ values at given wavelengths, along with fractional $95 \%$ confidence limits.

Please select the condition to analyze: condition:- "E3"

Are we writing out results to file? (1 for $y)$ : write_bool:=0

Read in data:

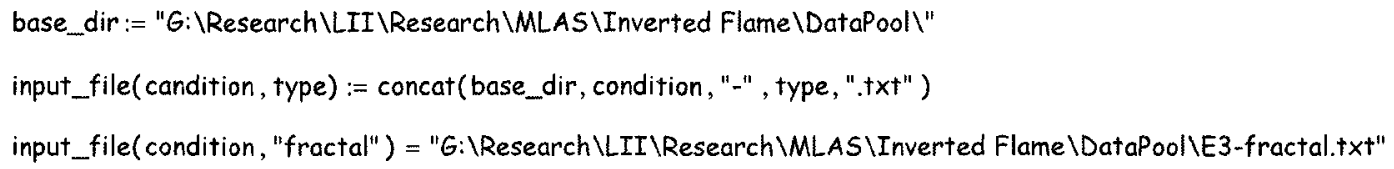

M1, $\tau$, residual for best, lower, upper $95 \%$.

$$
\begin{aligned}
& \text { SEM fractal: TEM result: Lognormal: } \\
& D f:=\left(\text { fractal }_{1}^{\langle i}\right)_{1}=1.701 \quad d_{p}:=d p_{1} n m=43.303 \cdot \mathrm{nm} \quad \mathrm{Ng}:=L N_{1,1}=106.52 \\
& k f:=\left(\text { fractal }^{\langle 1\rangle}\right)_{2}=7.222 \\
& \sigma 2 g:=\mathrm{LN}_{2,1}=2.633 \\
& M 1:=S P_{1,1}=120.66 \\
& T:=S P_{2,1}=0.13
\end{aligned}
$$

Adding in FM/EM ratio as a function of wavelength from Krishnan data. Using Table 3 from Krishnan, 2000, I get the following correlation (in the visible, 350-633)

$$
\operatorname{FMEM}(\lambda):=0.9118 \cdot \ln (\lambda)-4.7198
$$

Using wavelength as the subscript for easy indexing.

$$
\lambda_{\text {set }}:=450,475 . .950 \quad \text { FMEM }{\lambda_{\text {set }}}:=\operatorname{FMEM}\left(\lambda_{\text {set }}\right)
$$


Calculate the scatter-to-absorption ratio:

Best fit values:

$$
\begin{aligned}
& \rho S A L N_{\lambda_{\text {set }}}:=\rho S A 2_{2} C H G\left(D f, \mathbf{k f}, \lambda_{\text {set }} \cdot \mathrm{nm}, \mathrm{d}_{\mathrm{p}}, \mathrm{Ng}, \sigma 2 \mathrm{\sigma g}, \mathrm{FMEM}{\lambda_{\text {set }}}\right) \\
& \rho S \mathrm{SP}_{\lambda_{\text {set }}}:=\rho S A 2_{2} \mathrm{CHGSP}\left(\mathrm{Df}, \mathrm{kf}, \lambda_{\text {set }} \cdot \mathrm{nm}, \mathrm{d}_{\mathrm{p}}, M 1, \tau, \mathrm{FMEM}{\lambda_{\text {set }}}\right)
\end{aligned}
$$

Find uncertainty on p.SA parametrically:

Lower $95 \%$ on fit

$$
\begin{aligned}
& \rho S A L N . l f i{ }_{\lambda_{\text {set }}}:=\rho S A 2_{2} C H G\left(D f, k f, \lambda_{\text {set }} \bullet n m, d_{p}, L N_{1,2}, L N_{2,2}, F M E M_{\lambda_{\text {set }}}\right) \\
& \rho S A S P . I f f_{\lambda_{\text {set }}}:=\rho S A 2_{2} C H G S P\left(D f, k f, \lambda_{\text {set }} \cdot n m, d_{p}, S P_{1,2}, S P_{2,2}, F M E M_{\lambda_{\text {set }}}\right)
\end{aligned}
$$

Upper $95 \%$ on fit

$$
\begin{aligned}
& \rho S \text { LN.ufit }_{\lambda_{\text {set }}}:=\rho S A 2_{2} C H G\left(D f, \mathrm{kf}, \lambda_{\text {set }} \cdot \mathrm{nm}, d_{p}, \mathrm{LN}_{1,3}, \mathrm{LN}_{2,3}, \mathrm{FMEM}_{\lambda_{\text {set }}}\right) \\
& \rho S A \text { SP.u.fit } \\
& \lambda_{\text {set }}:=\rho S A 2_{2} C H G S P\left(D f, \mathrm{kf}, \lambda_{\text {set }} \cdot \mathrm{nm}, d_{p}, S P_{1,3}, S P_{2,3}, F M E M_{\lambda_{\text {set }}}\right)
\end{aligned}
$$

Lower $95 \%$ on dp

$$
\begin{gathered}
d p l i m:=d p_{1} \cdot \frac{d p_{2}}{100} \cdot n m=2.468 \times 10^{-9} \mathrm{~m} \quad \text { Mean *\%error } \\
\rho S A L N .1 . d p_{\lambda_{\text {set }}}:=\rho S A 2_{2} C H G\left[D f, k f, \lambda_{\text {set }} \cdot n m,\left(d_{p}-d p l i m\right), N g, \sigma 2 g, F M E M_{\lambda_{\text {set }}}\right] \\
\rho S A S P . I . d p_{\lambda_{\text {set }}}:=\rho S A 2 \_C H G S P\left[D f, k f, \lambda_{\text {set }} \cdot n m,\left(d_{p}-d p l i m\right), M 1, \tau, F M E M_{\lambda_{\text {set }}}\right]
\end{gathered}
$$

Upper $95 \%$ on dp

$$
\begin{aligned}
& p S A L N . u . d p_{\lambda_{\text {set }}}:=\rho S A 2_{2} C H G\left[D f, \mathrm{kf}, \lambda_{\text {set }} \cdot \mathrm{nm},\left(d_{p}+d_{p l i m}\right), N g, \sigma 2 g, F M E M_{\lambda_{\text {set }}}\right] \\
& p S A S P . d_{\lambda_{\text {set }}}:=p S A 2_{2} C H G S P\left[D f, k f, \lambda_{\text {set }} \cdot n m,\left(d_{p}+d p l i m\right), M 1, \tau, F M E M_{\lambda_{\text {set }}}\right]
\end{aligned}
$$

Lower $95 \%$ on fractal params

$$
\begin{aligned}
& \rho S A L N . I \text { frac }_{\lambda_{\text {set }}}:=\rho S A 2_{2} C H G\left(\text { fractal }_{1,2}, \text { fractal }_{2,2}, \lambda_{\text {set }} \cdot n m, d_{p}, N_{g}, \sigma 2 g, F_{E M} \lambda_{\lambda_{\text {set }}}\right)
\end{aligned}
$$

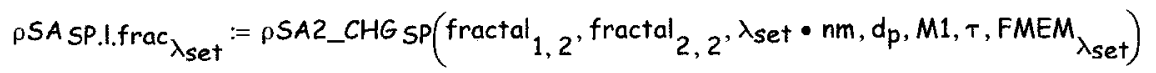

Upper $95 \%$ on fractal params

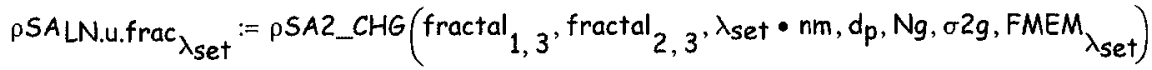

$$
\begin{aligned}
& \rho S A S P . u . \text { frac }_{\lambda_{\text {set }}}:=\rho S A 2_{2} C H G_{S P}\left(\text { fractal }_{1,3}, \text { fractal }_{2,3}, \lambda_{\text {set }} \cdot n m, d_{p}, M 1, \tau, F M E M_{\lambda_{\text {set }}}\right)
\end{aligned}
$$


Estimated fractional uncertainty on $\rho . s a$ is (with $10 \%$ added for RDG approx):

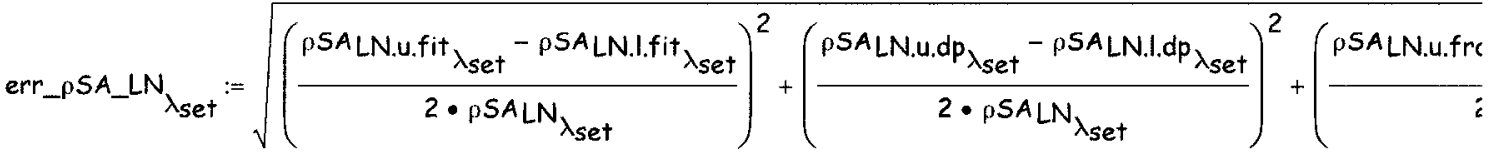

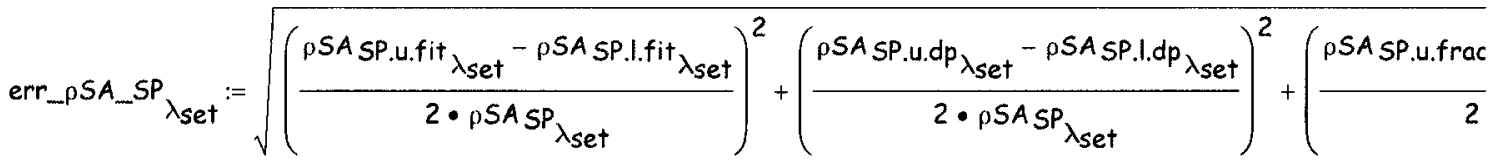

Note that these values represent the average

$$
e_{\text {avg }}=\frac{e_{h}+e \mid}{2}=\frac{\frac{x h-x}{x}+\frac{x-x \mid}{x}}{2}=\frac{x h-x \mid}{2 x}
$$

Tabulated results:

Lognormal:

\begin{tabular}{|c|c|c|c|}
\hline$\lambda_{\text {set }}=$ & FMEM $_{\lambda_{\text {set }}}$ & $\rho S A L N_{\lambda_{\text {set }}}=$ & $e r r_{-} \rho S A_{-}$ \\
\hline 450 & 0.851 & 0.3688 & 0.187 \\
\hline 475 & 0.9 & 0.3579 & 0.189 \\
\hline 500 & 0.947 & 0.3467 & 0.192 \\
\hline 525 & 0.991 & 0.3355 & 0.194 \\
\hline 550 & 1.034 & 0.3244 & 0.197 \\
\hline 575 & 1.074 & 0.3135 & 0.199 \\
\hline 600 & 1.113 & 0.3029 & 0.202 \\
\hline 625 & 1.15 & 0.2926 & 0.204 \\
\hline 650 & 1.186 & 0.2827 & 0.206 \\
\hline 675 & 1.22 & 0.2732 & 0.209 \\
\hline 700 & 1.253 & 0.264 & 0.211 \\
\hline 725 & 1.285 & 0.2552 & 0.214 \\
\hline 750 & 1.316 & 0.2468 & 0.216 \\
\hline 775 & 1.346 & 0.2387 & 0.218 \\
\hline 800 & 1.375 & 0.2309 & 0.221 \\
\hline 825 & 1.403 & 0.2235 & 0.223 \\
\hline 850 & 1.431 & 0.2164 & 0.226 \\
\hline 875 & 1.457 & 0.2096 & 0.228 \\
\hline 900 & 1.483 & 0.2031 & 0.231 \\
\hline 925 & 1.508 & 0.1969 & 0.233 \\
\hline 950 & 1.532 & 0.1909 & 0.236 \\
\hline
\end{tabular}

Best Frac. error:
Self-preserving:

\begin{tabular}{|c|c|}
\hline${ }_{S A} S P_{\lambda_{\text {se }}}$ & err_ $\rho S A$ \\
\hline 0.3481 & 0.191 \\
\hline 0.3372 & 0.193 \\
\hline 0.3261 & 0.196 \\
\hline
\end{tabular}

Best Frac.error: 


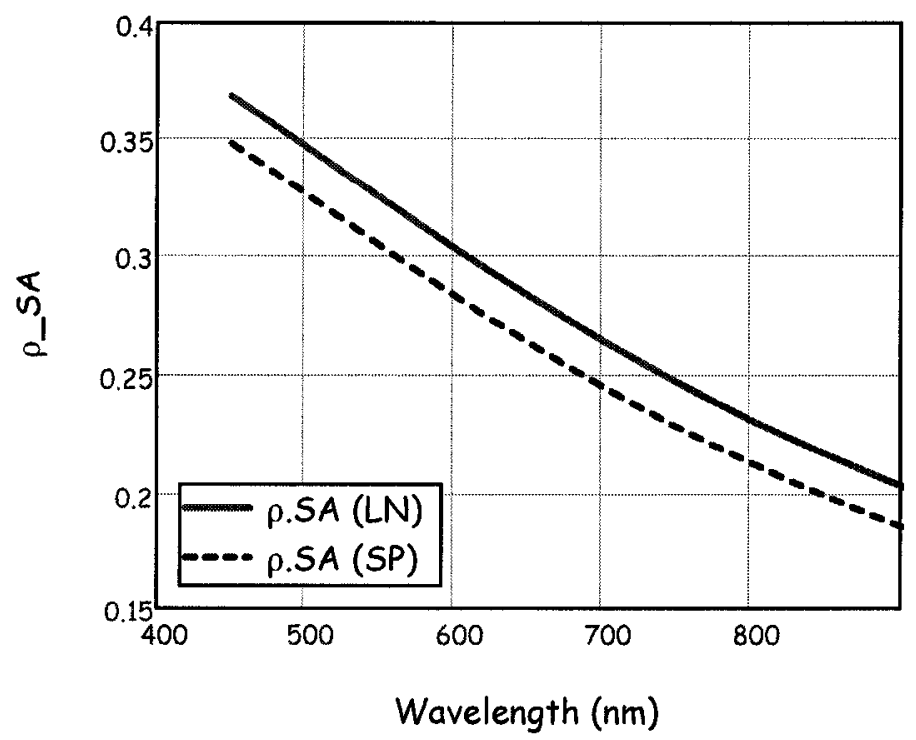

\section{Output Section:}

It turns out that having the wavelength as the array index means I have to re-index for output looping:

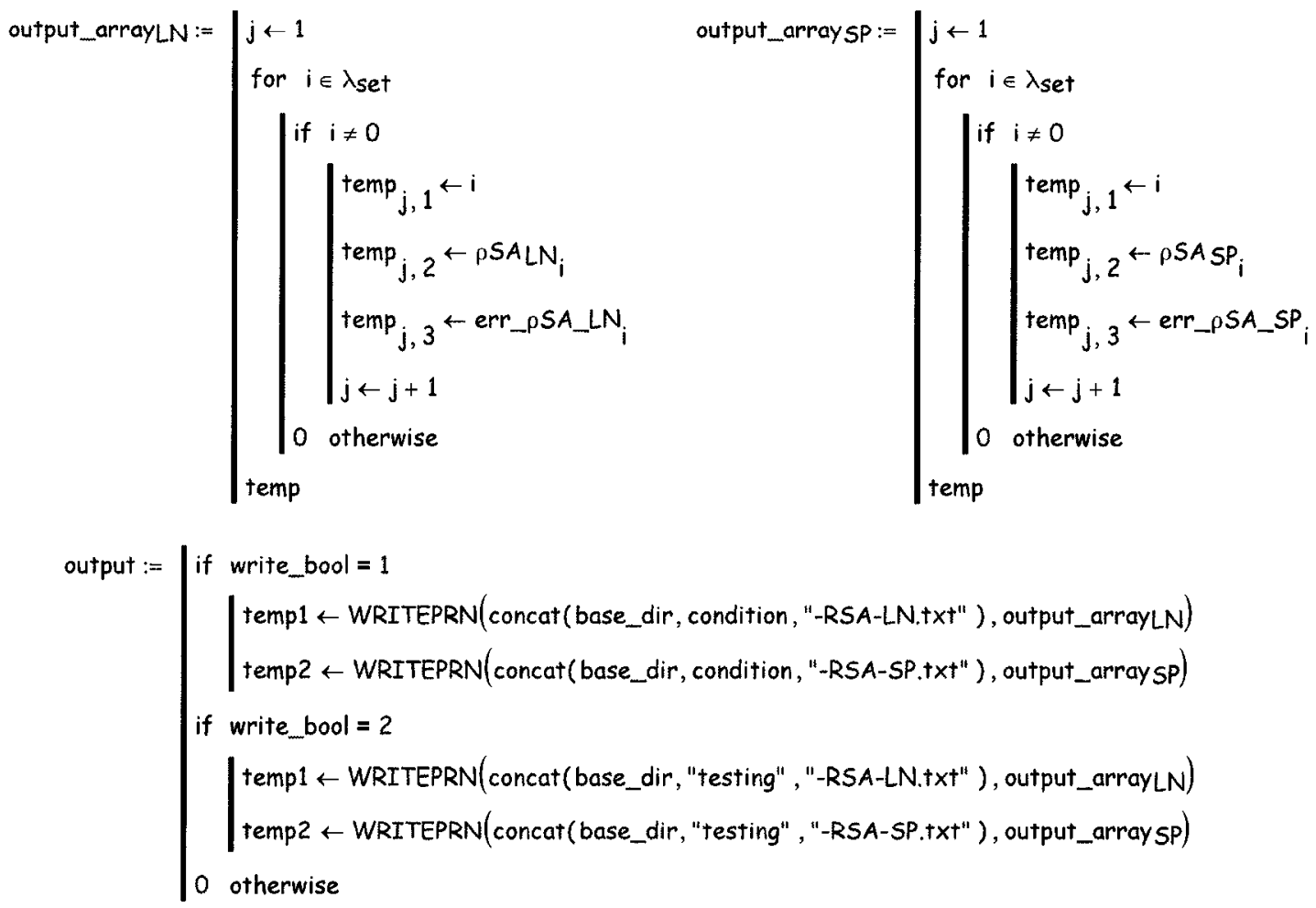




\section{B.3 LOSA Analysis}

The following Mathcad LOSA analysis sheet was adapted from the work of Drs. Snelling and Thomson at the NRC. Inputs are the raw images (plume, lamp, dark) for the condition and data range being analyzed. Outputs are tables of volumetric extinction coefficient and associated fractional uncertainty at 25-nm increments, as well as tables of spatially- and spectrally-resolved values of the optical thickness $(-\ln (\tau))$. The following steps are performed in the analysis:

- Read in images, bin data spatially and spectrally.

- Calculate the transmissivity, and normalize data with respect to the unattenuated portion on the left hand side of the images.

- Locate the centre of the plume, such that data from the left- and right-hand sides can be analyzed separately.

- Smooth data using least squares regression, convert transmissivity to optical thickness in preparation for tomographic inversion.

- Invert the data to obtain a radial transmissivity profile.

- Find the mean volumetric extinction coefficient and associated propagated uncertainty from the central section with radii from 0 to $5 \mathrm{~mm}$.

- Output tables of extinction coefficient and optical thickness for use in later analyses. 


\section{Spectral line-of-sight attenuation analysis: volumetric extinction coefficient}

This sheet has been adapted from the LOSA analysis sheet developed by Drs Snelling and Thomson.

Enter the name of the data set to run $(\mathrm{Al}, \mathrm{B} 1$, etc): condition := "Al"

Enter wavelength range $-\mathrm{h}$ for high (filtered), I for low (unfiltered): set := "1"

Turn on file writing by setting this to 1 : write bool $:=0$

Set the filenames:

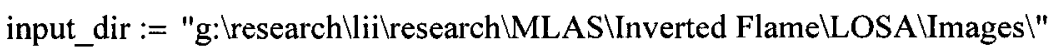

input_file(what, type) $:=$ concat(input_dir, what, "-" , set, "-" ,type, ".SPE")

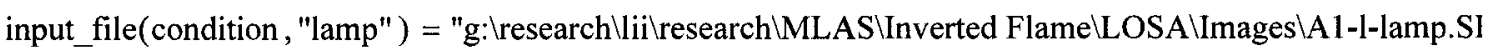

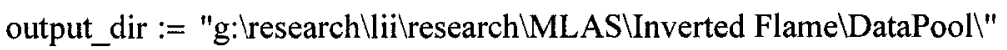

output_file(label) := concat(output_dir, condition, "-" , set, "-" , label, ".txt" )

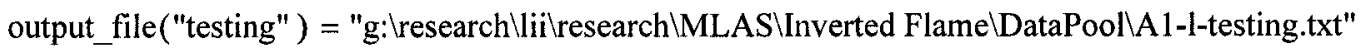

Read in the header from a representative image to determine data type and image dimensions

$$
\begin{aligned}
& \text { datatypes }:=\left(\begin{array}{c}
\text { "float" } \\
\text { "uint32" } \\
\text { "float" } \\
\text { "uint16" }
\end{array}\right) \text { datasizes : }=\left(\begin{array}{l}
4 \\
4 \\
4 \\
2
\end{array}\right) \\
& \text { headersize }:=4100 \\
& \text { header := READBIN(input_file(condition, "lamp" ), "uint16" , 0, 1,0, } \left.\frac{\text { headersize }}{2}\right)
\end{aligned}
$$

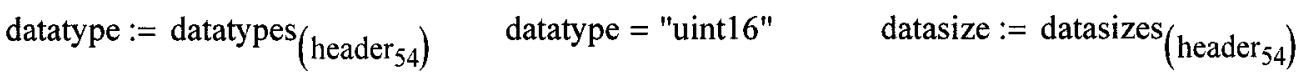

$$
\begin{aligned}
& \text { pwidth }:=\text { header }_{21} \quad \text { pheight }:=\text { header }_{328} \quad \text { num_frames }:=\text { header }_{723} \\
& \text { pwidth }=1 \times 10^{3} \quad \text { pheight }=400 \quad \text { num_frames }=30 \\
& \operatorname{cropx} 1:=125 \operatorname{cropx} 2:=\text { pwidth }-125 \text { cropy } 1:=0 \text { cropy } 2:=\text { pheight }-1 \\
& \text { offset(frame) }:=\text { headersize }+ \text { datasize pwidth.pheight frame } \\
& \text { image := submatrix(READBIN(input_file(condition, "lamp" ), datatype, } 0 \text {, pwidth, offset( } 0 \text { ), pheight), cropy }
\end{aligned}
$$


Preview a sample frame from the image:

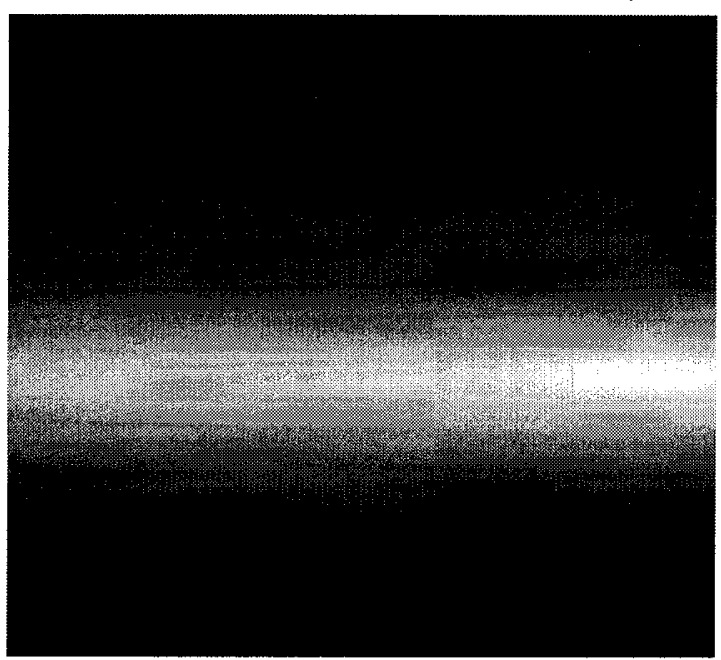

Bin the data by wavelength grouping and spatially and generate transmission curves

$$
\mathrm{m} \lambda:=-2.0214 \quad \mathrm{~b} \lambda:=1065.184 \quad \text { Spectral calibration information }
$$

$f \lambda($ pixel $):=m \lambda \cdot$ pixel $+b \lambda$

half_height $:=\operatorname{round}\left[12 \cdot\left(\sqrt{\mathrm{m} \lambda^{2}}\right)^{-1}\right] \quad$ half_height $=6 \quad$ total_height $:=$ half_height $\cdot 2+1$

$$
\begin{array}{lll}
\operatorname{pixel}(\lambda):=\operatorname{round}\left(\frac{\mathrm{b} \lambda-\lambda}{-\mathrm{m} \lambda}\right) & \operatorname{pixel}(500)=280 & \text { total_height }=13 \\
\operatorname{bin\_ top}(\lambda):=\operatorname{pixel}(\lambda)-\text { half_height } & \operatorname{bin\_ top}(1000)=26 & \mathrm{f} \lambda(0)=1.065 \times 10^{3} \\
& \mathrm{f} \lambda(325)=408.229
\end{array}
$$

bin_bottom $(\lambda):=\operatorname{pixel}(\lambda)+$ half_height

$$
\begin{aligned}
& \lambda \_ \text {start }:=\mid \begin{array}{l}
450 \text { if set }=\text { "l" } \\
675 \text { if } \text { set }=\text { "h" }
\end{array} \quad \lambda_{-} \text {end }:=\mid \begin{array}{l}
750 \text { if } \text { set }=\text { "l" } \\
950 \text { if set }=\text { "h" }
\end{array} \\
& \text { bin_bottom }\left(\lambda \_ \text {start }\right)=310
\end{aligned}
$$

$$
\mathrm{i}:=0 . . \text { total_height }-1 \quad \text { ones }_{\mathrm{i}}:=1
$$

$$
j:=0 . . \frac{\lambda \_ \text {end }-\lambda \_s t a r t}{\lambda \_ \text {inc }} \quad \lambda_{j}:=\lambda \_ \text {start }+\lambda \_ \text {inc } \cdot j
$$

Spatial calibration:

$$
\text { scale }:=\frac{20}{777-179}
$$

xbin $:=3 \quad \mathrm{j}:=0 . . x$ bin $-1 \quad$ ones $2_{j}:=1$

scale $=0.033$

$\mathrm{xbin} \cdot$ scale $=0.1 \quad \mathrm{~m} \lambda \cdot$ total_height $=-26.278$ 
Start reading in the data from multiple frames

$$
\begin{array}{ll}
\mathrm{j}:=0 . . \text { num_frames }-1 & \mathrm{x}:=0 . . \text { pwidth }-1 \\
& \mathrm{y}:=0 . . \text { pheight }-1
\end{array}
$$

Read in dark data:

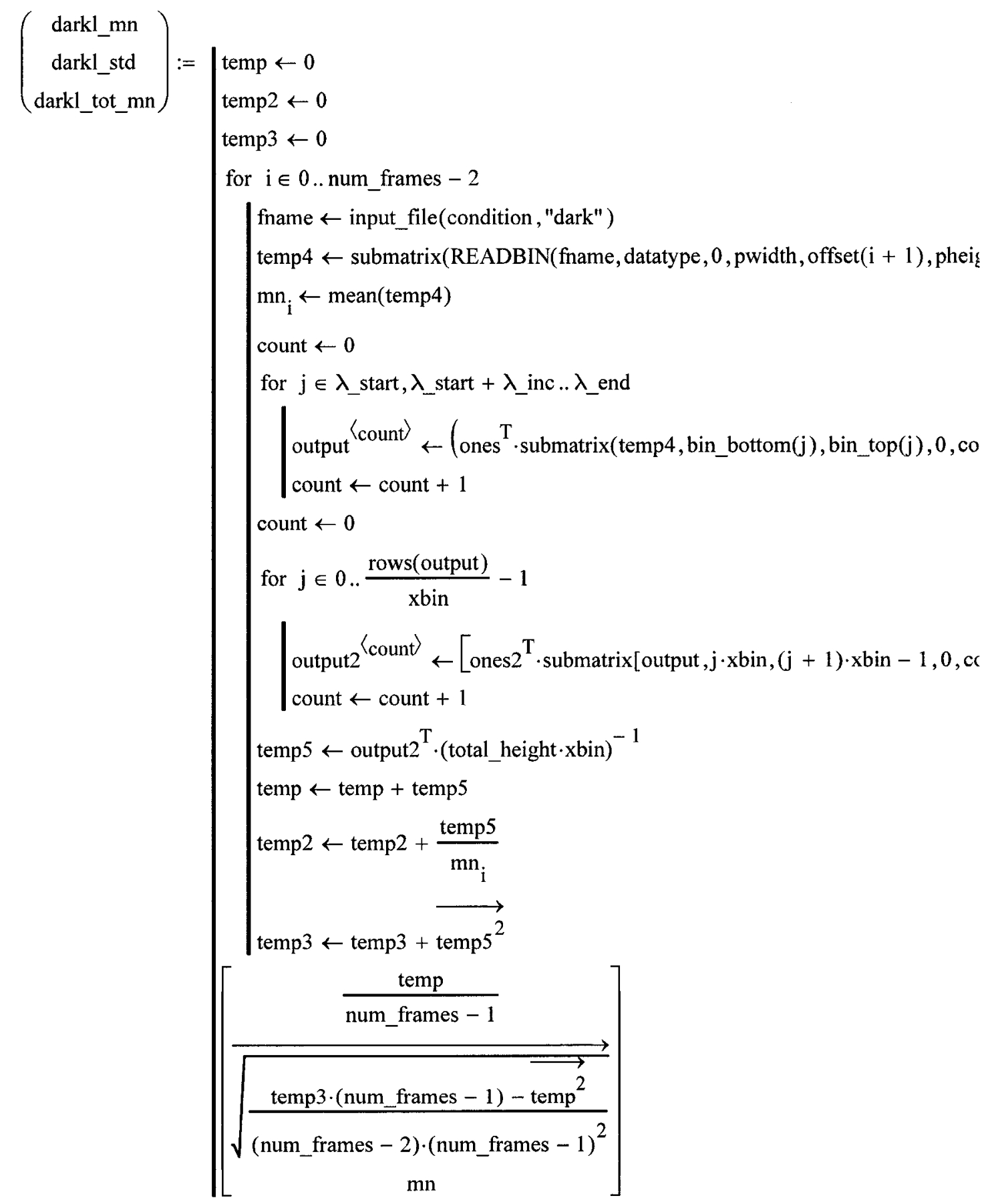

$$
\begin{aligned}
& \text { mean }\left(\overrightarrow{\frac{\text { darkl_std }}{\text { darkl_mn }}}\right)=5.345 \times 10^{-4} \frac{\text { Stdev(darkl_tot_mn })}{\text { mean }(\text { darkl_tot_mn }) \cdot \sqrt{\text { num_frames }}}=1.417 \times 10^{-4}
\end{aligned}
$$


mean $($ darkl_std $)=0.071$

Left in the calcs from the legacy sheet. No flame in this case, so the two dark images are equivalent.

$$
\left(\begin{array}{c}
\text { darkf_mn } \\
\text { darkf_std } \\
\text { darkf_tot_mn }
\end{array}\right):=\left(\begin{array}{c}
\text { darkl_mn } \\
\text { darkl_std } \\
\text { darkl_tot_mn }
\end{array}\right)
$$

Read in lamp data:

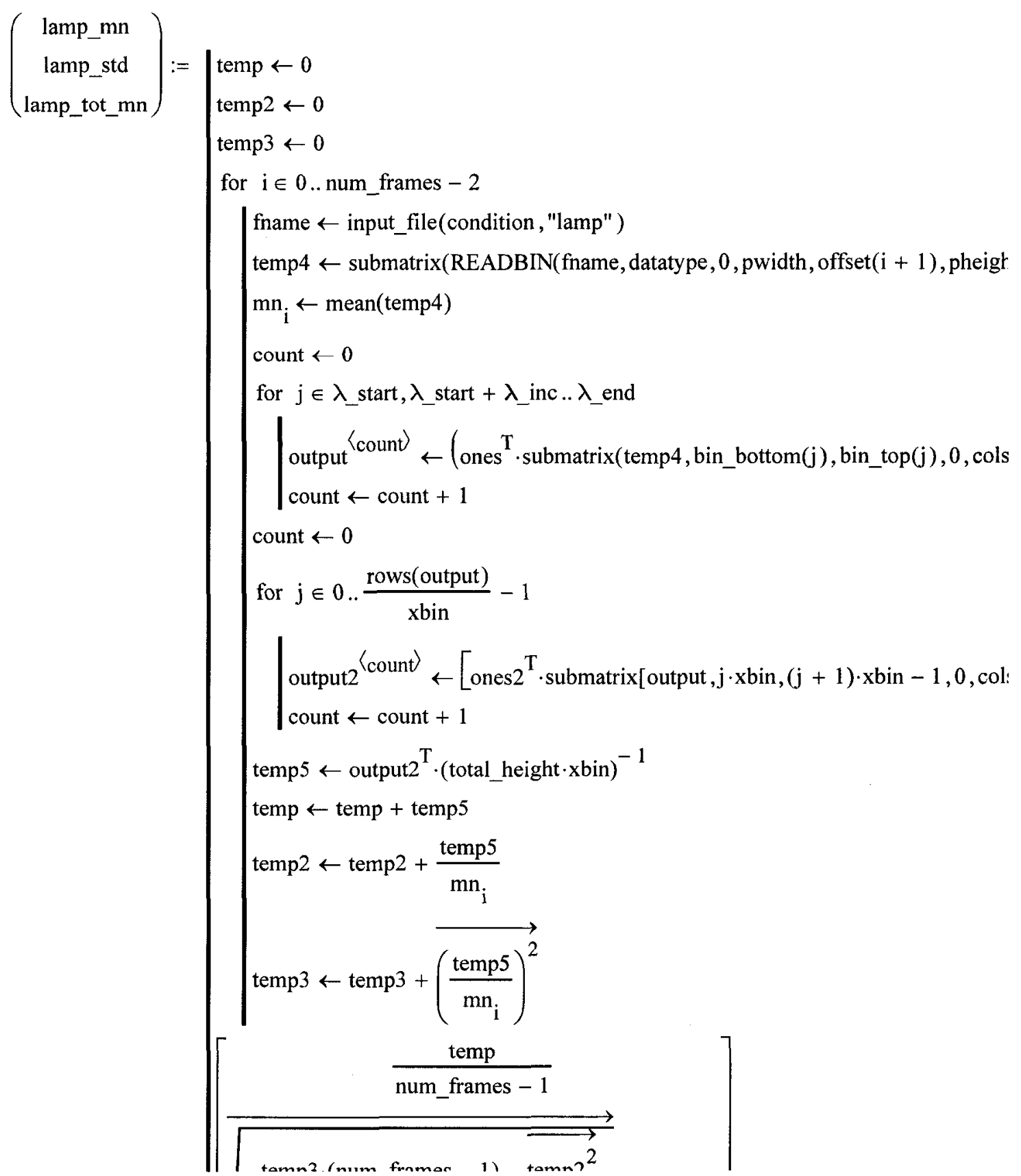




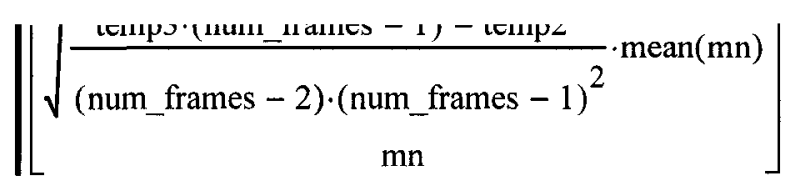

mean $\left(\overrightarrow{\frac{\text { lamp_std }}{\text { lamp_mn }}}\right)=9.082 \times 10^{-5} \quad \frac{\text { Stdev(lamp_tot_mn })}{\text { mean }(\text { lamp_tot_mn }) \cdot \sqrt{\text { num_frames }}}=3.194 \times 10^{-4}$

mean $($ lamp_std $)=2.556$

$\frac{1}{\sqrt{\text { mean(lamp_tot_mn }) \cdot 10 \cdot \text { num_frames } \cdot \text { total_height } \cdot \text { xbin }}}=7.429 \times 10^{-5}$

\section{Read in plume data:}

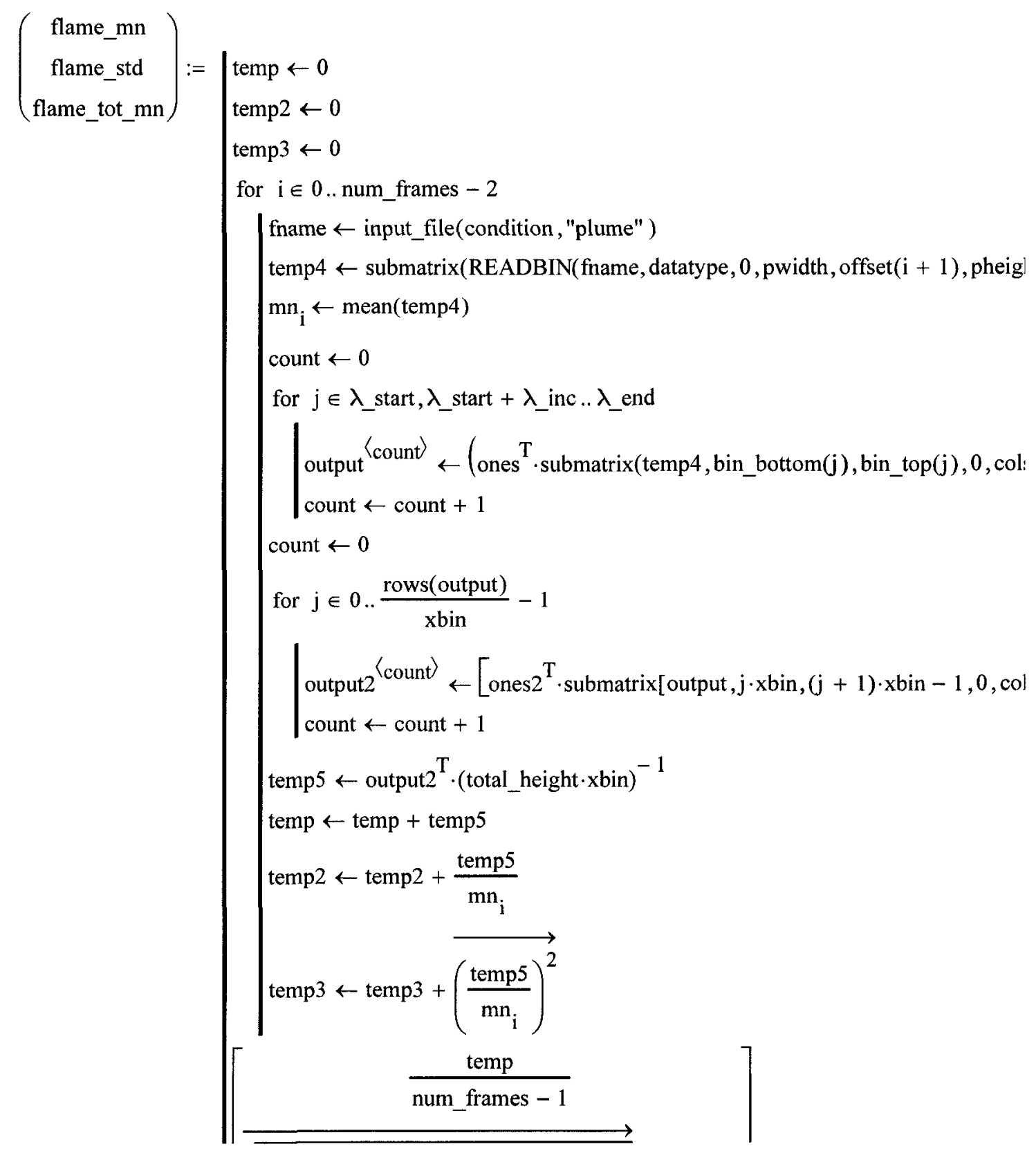




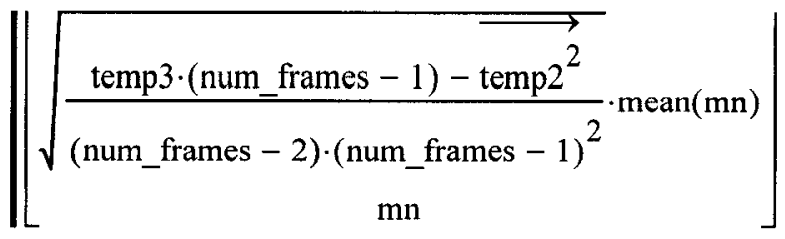

Run some quick stats:

$$
\begin{aligned}
& \text { mean }\left(\overrightarrow{\frac{\text { flame_std }}{\text { flame_mn }}}\right)=8.883 \times 10^{-5} \frac{\text { Stdev(flame_tot_mn })}{\text { mean(flame_tot_mn }) \cdot \sqrt{\text { num_frames }}}=2.275 \times 10^{-4} \\
& \text { mean }(\text { flame_std })=2.497 \quad \frac{1}{\sqrt{\text { mean(lamp_tot_mn }) \cdot 10 \cdot \text { num_frames } \cdot \text { total_height }}}=1.287 \times 10^{-4} \\
& \tau:=\frac{\overrightarrow{\text { flame_mn }- \text { darkf_mn }}}{\text { lamp_mn }- \text { darkl_mn }+0.0001}
\end{aligned}
$$

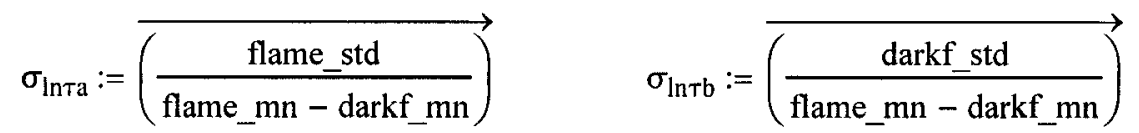

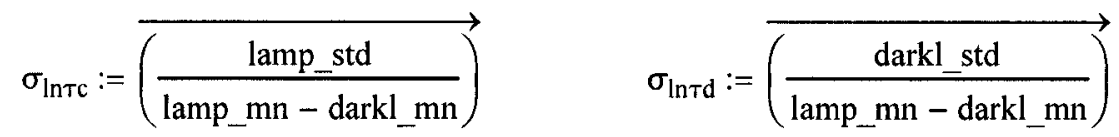

$$
\begin{aligned}
& \sigma_{\ln \tau 2}:=\sqrt{\overrightarrow{\sigma_{\ln \tau a}^{2}+\sigma_{\ln \tau b}^{2}+\sigma_{\ln \tau c}^{2}}+\overrightarrow{\sigma_{\ln \tau d}}} \quad \operatorname{mean}(\tau)=0.996 \\
& \operatorname{rows}(\tau)=250 \quad \operatorname{cols}(\tau)=13 \quad \mathrm{i}:=0 . . \operatorname{rows}(\tau)-1 \quad \text { ind }:=3
\end{aligned}
$$

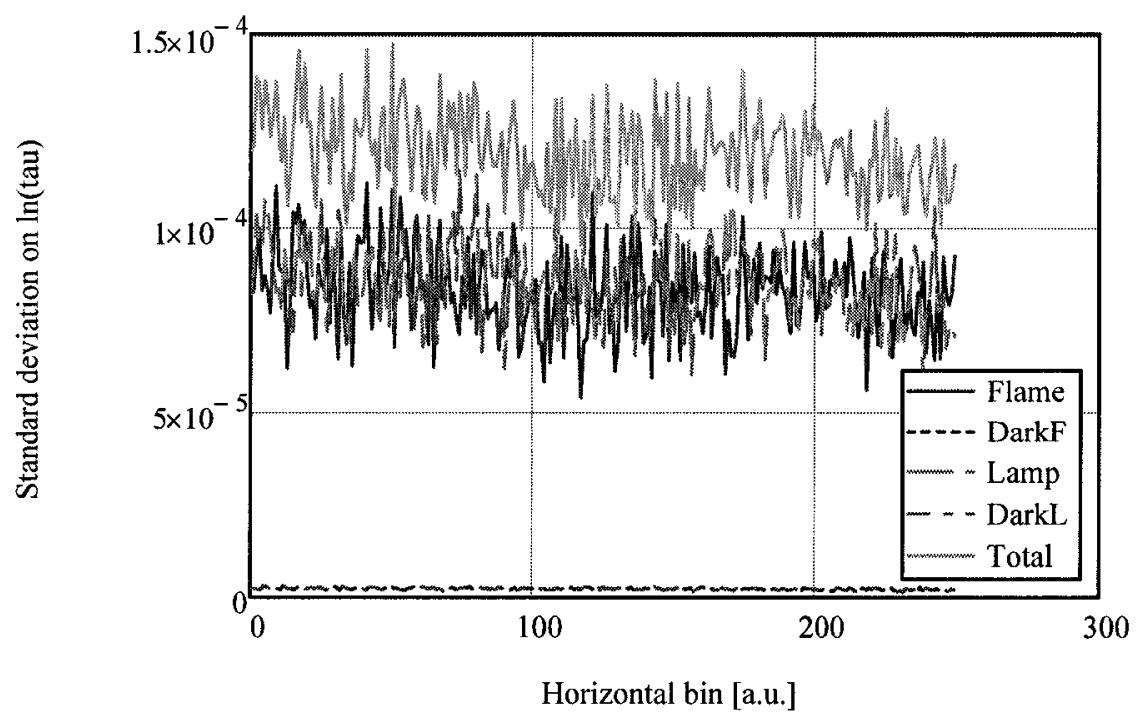


Raw transmissivity curves for selected wavelengths:

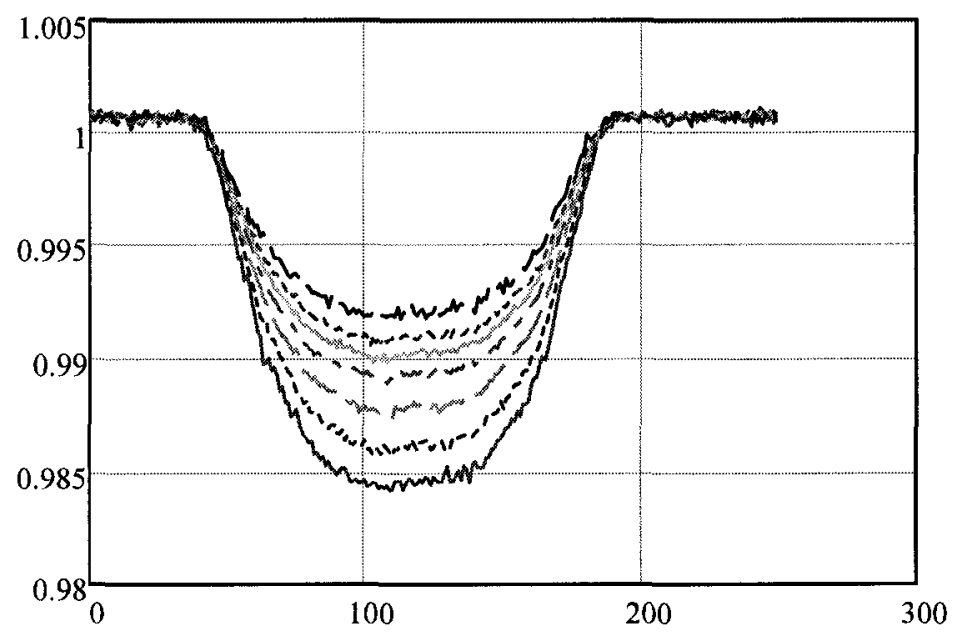

Define the unattenuated ranges

$$
\begin{array}{ll}
\text { LHS Left }:=\operatorname{round}\left(0 \cdot \mathrm{xbin}^{-1}\right)=0 & \text { RHS Left }:=\operatorname{round}(600 \mathrm{xbin}-1)=200 \\
\text { LHS_Right }:=\operatorname{round}\left(90 \cdot \mathrm{xbin}^{-1}\right)=30 & \text { RHS_Right }:=\operatorname{round}\left(750 \cdot \mathrm{xbin}^{-1}\right)-1=249
\end{array}
$$

Define the middle guess and make position array

$$
\begin{aligned}
& \text { CentreSeed }:=\operatorname{round}\left(375 \cdot \operatorname{xbin}^{-1}\right)=125 \\
& \mathrm{i}:=0 . \operatorname{rows}(\tau)-1 \quad \operatorname{pos}_{\mathrm{i}}:=\mathrm{i}
\end{aligned}
$$

Normalize the curves using the mean of the LHS region

$$
\begin{aligned}
& \text { set }:=0 . . \operatorname{cols}(\tau)-1 \quad \text { Normal }_{\text {set }}:=\operatorname{mean}(\operatorname{submatrix}(\tau, \text { LHS_Left,LHS_Right, set, set })) \\
& \operatorname{norm} \tau^{\langle\text {set }\rangle}:=\tau^{\langle\text {set }\rangle} \cdot\left(\text { Normal }_{\text {set }}\right)^{-1} \quad \text { radius }_{\mathrm{i}}:=\left(\mathrm{i}-\mathrm{154.75}^{-1} \cdot \mathrm{xbin} \cdot \mathrm{scale}\right. \\
& a:=0 \ldots \operatorname{rows}(\tau)-1 \\
& \text { Intau_write }:=-\overrightarrow{\ln (\text { norm } \tau)}
\end{aligned}
$$

Normalized transmission curves 


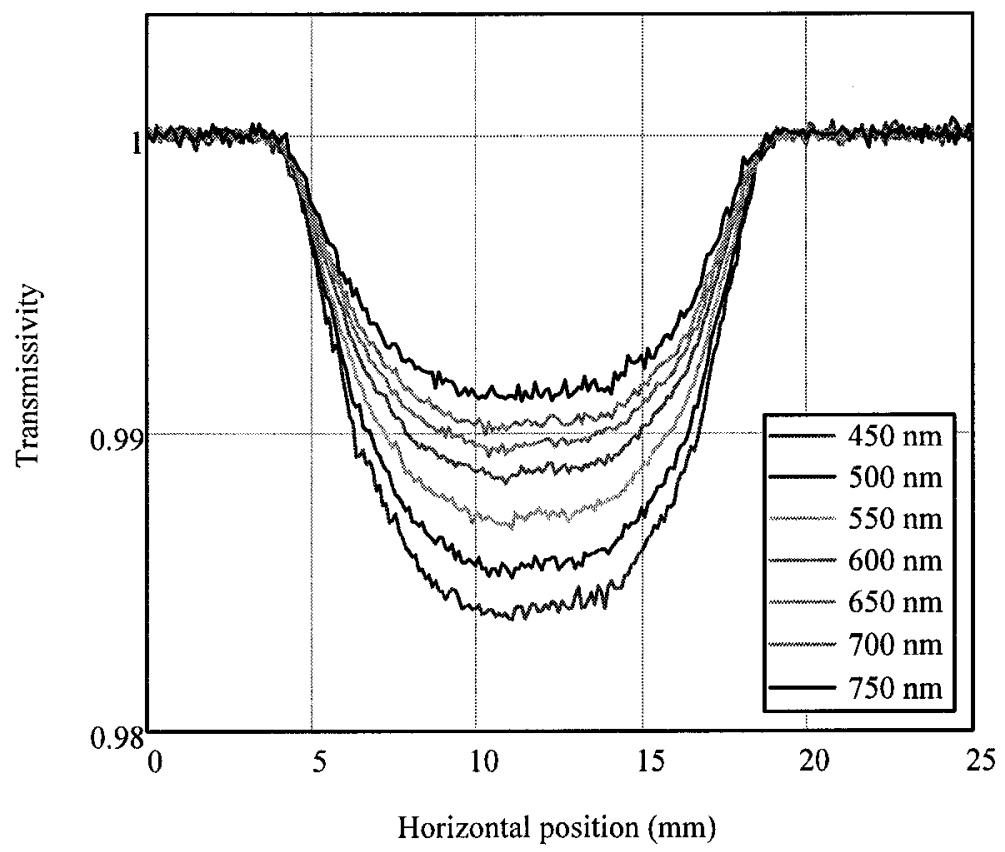

Visualization of the soot plume: $\operatorname{Tmax}:=1.005 \tau \min :=0.98$
$0 \mathrm{~mm}$
$25 \mathrm{~mm}$

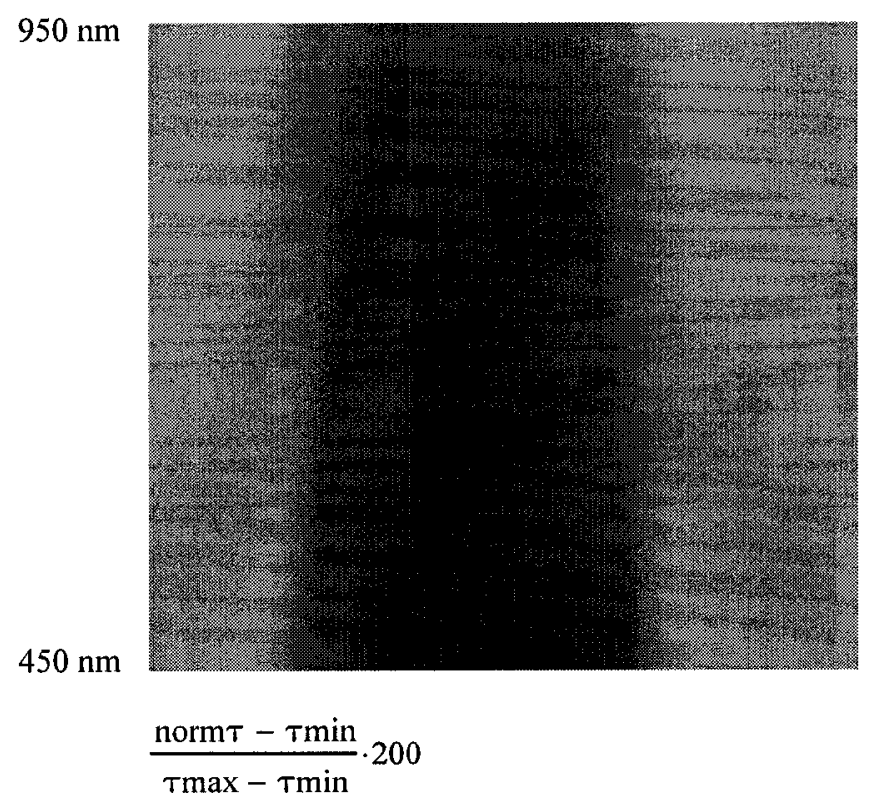

Calculate the stats on the LHS unattenuated region

set $:=0 . . \operatorname{cols}($ norm $\tau)-1$

$\mathrm{LHSmean}_{\text {set }}:=\operatorname{mean}(\operatorname{submatrix}($ norm $\tau$, LHS_Left,LHS_Right, set, set)) 
$\operatorname{LHSstdev}_{\text {set }}:=\operatorname{stdev}($ submatrix(norm $\tau$, LHS_Left, LHS_Right, set, set))

LHSstdmean $\tau:=$ LHSstdev $\tau \cdot(\text { LHS_Right }- \text { LHS_Left }+1)^{-0.5}$

Calculate the stats on the RHS unattenuated region

$$
\begin{aligned}
& \text { RHSmean }_{\text {set }}:=\text { mean(submatrix(norm }, \text { RHS_Left, RHS_Right, set, set)) }
\end{aligned}
$$

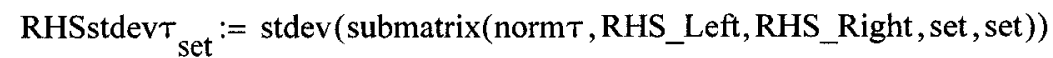

$$
\begin{aligned}
& \text { RHSstdmean } \tau:=\text { RHSstdev } \tau \cdot(\text { RHS_Right }- \text { RHS_Left }+1)^{-0.5} \\
& \text { LHSmean } \tau=\begin{array}{|r|r|}
\hline & 0 \\
\hline 0 & 1 \\
\hline 1 & 1 \\
\hline 2 & 1 \\
\hline 3 & 1 \\
\hline 4 & 1 \\
\hline 5 & 1 \\
\hline 6 & 1 \\
\hline 7 & 1 \\
\hline 8 & 1 \\
\hline 9 & 1 \\
\hline 10 & 1 \\
\hline 11 & 1 \\
\hline 12 & 1 \\
\hline
\end{array}
\end{aligned}
$$

\begin{tabular}{|c|c|c|}
\hline & & 0 \\
\hline & 0 & $3.8 \cdot 10^{-5}$ \\
\hline & 1 & $2.841 \cdot 10^{-5}$ \\
\hline & 2 & $2.533 \cdot 10^{-5}$ \\
\hline & 3 & $2.404 \cdot 10^{-5}$ \\
\hline & 4 & $2.1 \cdot 10^{-5}$ \\
\hline & 5 & $1.891 \cdot 10^{-5}$ \\
\hline 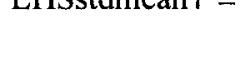 & 6 & $1.326 \cdot 10^{-5}$ \\
\hline & 7 & $2.05 \cdot 10^{-5}$ \\
\hline & 8 & $2.071 \cdot 10^{-5}$ \\
\hline & 9 & $1.805 \cdot 10^{-5}$ \\
\hline & 10 & $1.873 \cdot 10^{-5}$ \\
\hline & 11 & $2.959 \cdot 10^{-5}$ \\
\hline & 12 & $2.751 \cdot 10^{-5}$ \\
\hline
\end{tabular}

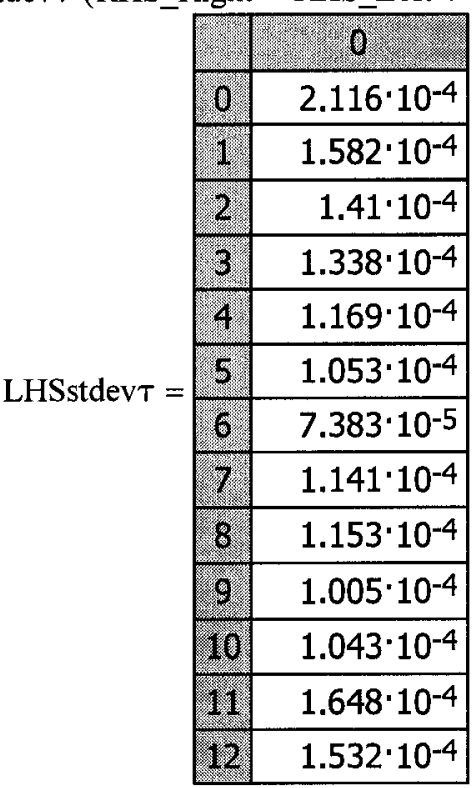

RHSmean $\tau=$\begin{tabular}{|r|r|}
\hline & 0 \\
\hline 0 & 1 \\
\hline 1 & 1 \\
\hline 2 & 1 \\
\hline 3 & 1 \\
\hline 4 & 1 \\
\hline 5 & 1 \\
\hline 6 & 1 \\
\hline 7 & 1 \\
\hline 8 & 1 \\
\hline 9 & 1 \\
\hline 10 & 1 \\
\hline 11 & 1 \\
\hline 12 & 1 \\
\hline
\end{tabular}

\begin{tabular}{|c|c|}
\hline & 0 \\
\hline 0 & $2.087 \cdot 10^{-4}$ \\
\hline 1 & $1.529 \cdot 10^{-4}$ \\
\hline 2 & $1.302 \cdot 10^{-4}$ \\
\hline 3 & $1.009 \cdot 10^{-4}$ \\
\hline 4 & $1.036 \cdot 10^{-4}$ \\
\hline 5 & $6.165 \cdot 10^{-5}$ \\
\hline 6 & $7.557 \cdot 10^{-5}$ \\
\hline 7 & $7.686 \cdot 10^{-5}$ \\
\hline 8 & $8.642 \cdot 10^{-5}$ \\
\hline 9 & $8.546 \cdot 10^{-5}$ \\
\hline 10 & $1.186 \cdot 10^{-4}$ \\
\hline 11 & $1.46 \cdot 10^{-4}$ \\
\hline 12 & $1.544 \cdot 10^{-4}$ \\
\hline
\end{tabular}

\begin{tabular}{|c|c|}
\hline & 0 \\
\hline 0 & $2.952 \cdot 10^{-5}$ \\
\hline 1 & $2.162 \cdot 10^{-5}$ \\
\hline 2 & $1.842 \cdot 10^{-5}$ \\
\hline 3 & $1.427 \cdot 10^{-5}$ \\
\hline 4 & $1.465 \cdot 10^{-5}$ \\
\hline 5 & $8.719 \cdot 10^{-6}$ \\
\hline 6 & $1.069 \cdot 10^{-5}$ \\
\hline 7 & $1.087 \cdot 10^{-5}$ \\
\hline 8 & $1.222 \cdot 10^{-5}$ \\
\hline 9 & $1.209 \cdot 10^{-5}$ \\
\hline 10 & $1.678 \cdot 10^{-5}$ \\
\hline 11 & $2.064 \cdot 10^{-5}$ \\
\hline 12 & $2.184 \cdot 10^{-5}$ \\
\hline
\end{tabular}




\section{Data Centring}

We first smooth the data so that the algorithm works at low burner heights with large signal to noise ratios. If we didn't do this, the algorithm would "jump out" of the loop if the noise crossed the "half max" line. The reason it is smoothed is to eliminate or smooth the noise without comprimizing the shape of the intensity. The variable span can be adjusted to get better fits. The variable Centreseed is an estimate of burner center and was defined at the top of the worksheet.

Display the CentreSeed variable

CentreSeed $=125$

Define range variable sets

$$
\text { sets }:=0 . . \operatorname{cols}(\tau)-1
$$

Define argument span used by the Loess function $\quad$ span $:=0.1$

Use the loess function to define vector vs required by interp function

$$
\text { vs }^{\langle\text {sets }\rangle}:=\operatorname{loess}\left(\text { pos, norm } \tau^{\langle\text {sets }\rangle}\right. \text {, span) }
$$

Create a new matrix of Smoothed data using the interp function

$$
\text { Smoothed }(x, \text { sets }):=\operatorname{interp}\left(v^{\langle}{ }^{\langle\text {sets }\rangle}, \text { pos, } \tau^{\langle\text {sets }\rangle}, \mathrm{x}\right)
$$

$$
\text { CLtol }:=.001 \quad \text { Cinc }:=0.1
$$

For each vertical height calculate a new midpoint. The midpoint is defined as the radial position half way between the left and right radial position for which the transmission value has dropped $1 / 3$ of the way to the minimum. This code uses the variable Centreseed as an estimate of burner center where the minimum transmission should be.

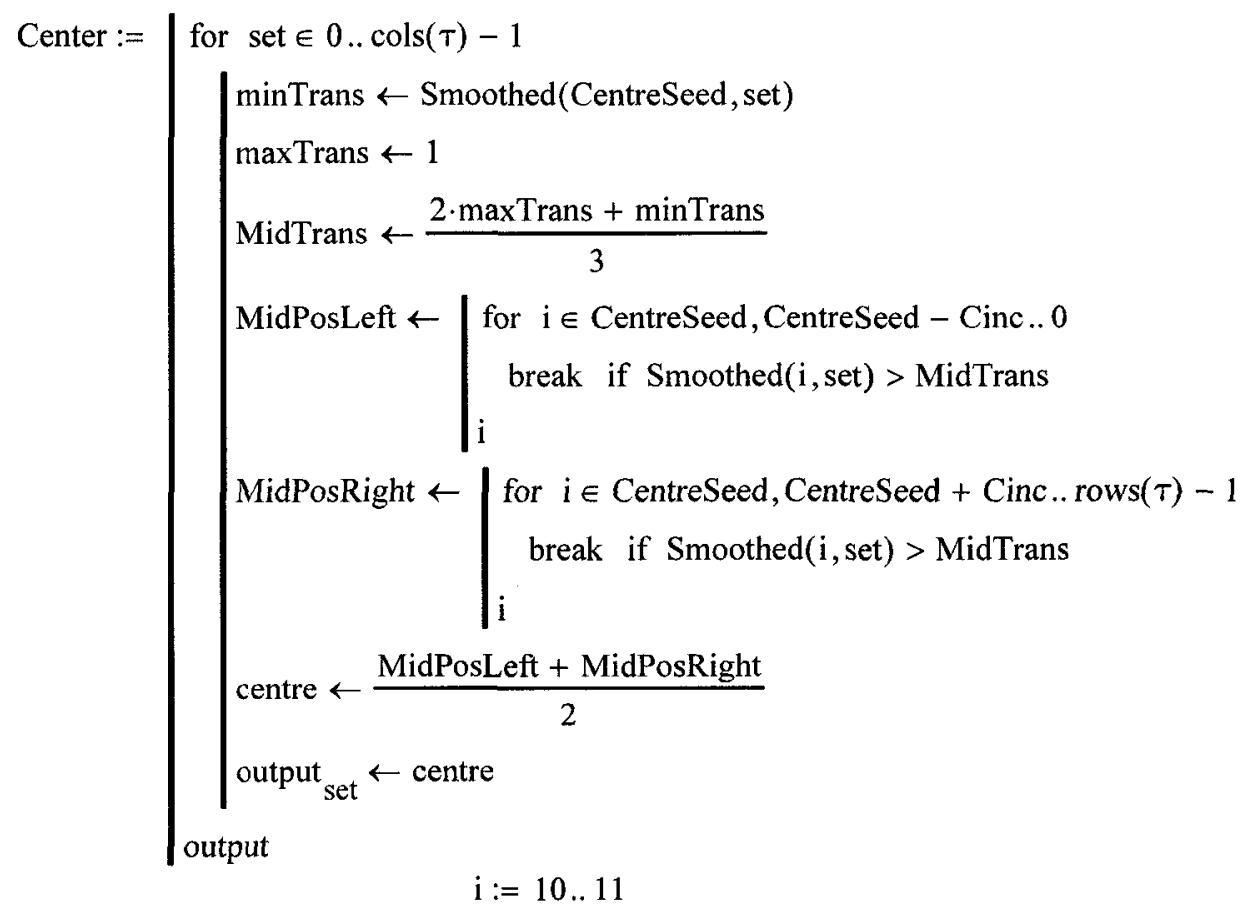




\section{Loess Smooth the Data}

We want to generate smoothed transmission curves which run from flame centre out. We can generate both left and right side curves. If the transmission data is noisy then analyze the noisy data with a higher LoessPar. Define the two Loess parameters (LoessParLQ and LoessParHQ) and change the cutoff point with Iqend.

\section{Smoothing Global Variables}

\section{Data Radial Increment}

$$
\text { Dinc }:=1
$$

$$
\begin{aligned}
& \text { Loess Smooth } \\
& \text { Parameters } \\
& \text { LoessParLQ }:=0.05 \\
& \text { LoessParHQ }:=\text { LoessParLQ }
\end{aligned}
$$

\section{Radial \\ Points}

RadLim : $=$ round $\left(\frac{300}{x b i n}\right)$

Break Data into HQ and LQ and smooth accordingly. Iqstart and lqend define the first and last height to be smoothed with LoessParLQ parameter. Increase the Loess parameter for noisy data range. Set1 uses the LoessParLQ parameter and Set2 uses the LoessParHQ parameter. Remember low set indexes correspond to strips with higher vertical heights. The nosiy data usually occurs at locations where there is very little soot - near the burner tip and near the tip of the flame.t

$$
\begin{aligned}
& \text { lqstart }:=0 \quad \text { lqend }:=8 \quad \text { hqend }:=\operatorname{cols}(\operatorname{norm} \tau)-1 \\
& \mathrm{i}:=0 . . \operatorname{cols}(\text { norm } \tau)-1 \quad \operatorname{span}_{\mathrm{i}}:=\text { if }(\mathrm{i}>\text { lqend, LoessParHQ, LoessParLQ }) \\
& \operatorname{pos} 2:=\text { pos } \cdot \text { scale } \cdot x b i n \quad \text { Center } 2:=\text { Center } \cdot \text { scale } \cdot x \text { bin } \quad \text { scale } \cdot x b i n=0.1 \quad \text { int }:=\text { scale } \cdot x b i n
\end{aligned}
$$

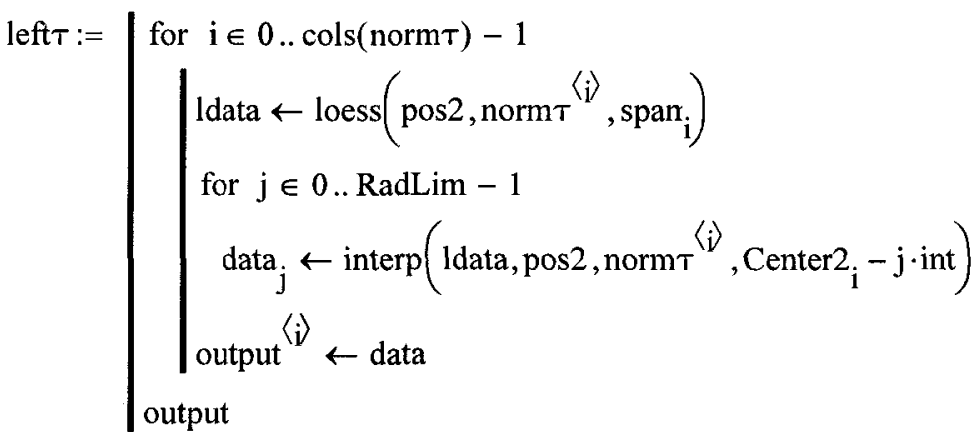

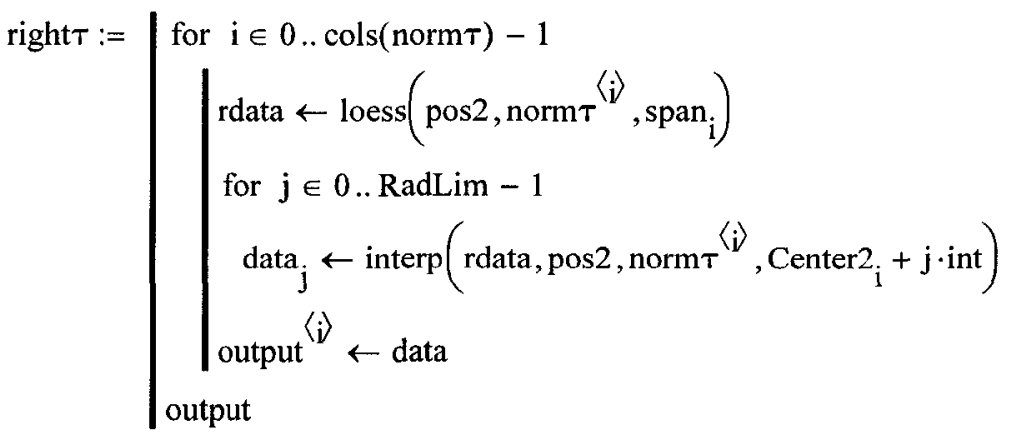




$$
\begin{aligned}
& \operatorname{left} \sigma \ln \tau:=\mid \text { for } i \in 0 . . \operatorname{cols}(\operatorname{norm} \tau)-1 \\
& \text { Idata } \leftarrow \operatorname{loess}\left(\operatorname{pos} 2, \sigma_{\ln \tau 2}\langle i\rangle, \operatorname{span}_{i}\right) \\
& \text { for } \mathrm{j} \in 0 \text {... RadLim - } 1 \\
& \text { data }_{\mathrm{j}} \leftarrow \text { interp }\left(\text { ldata, pos } 2, \sigma_{\ln \tau 2}\langle i\rangle, \text { Center } 2_{i}-\mathrm{j} \cdot \text { int }\right) \\
& \text { output }^{\langle i\rangle} \leftarrow \text { data } \\
& \text { output }
\end{aligned}
$$

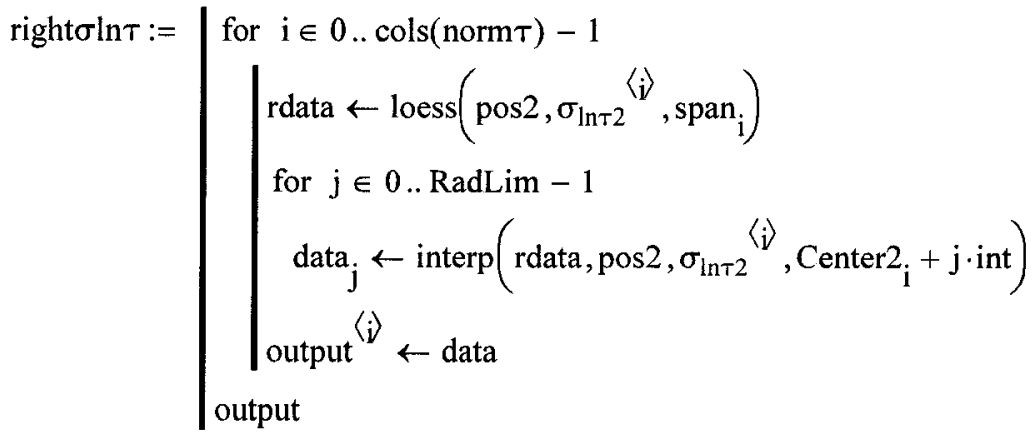

$\mathrm{i}:=0 .$. RadLim -1

Smoothed Transmissivity Data

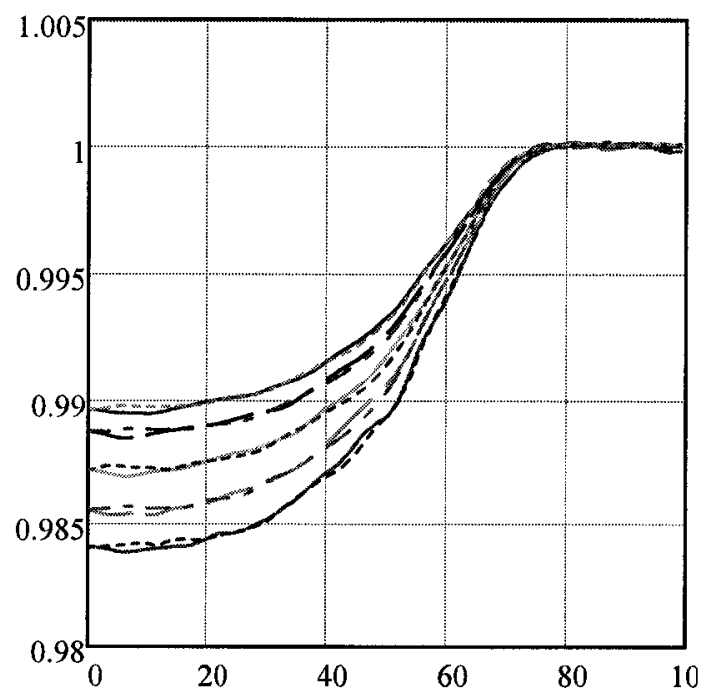

Standard Deviations

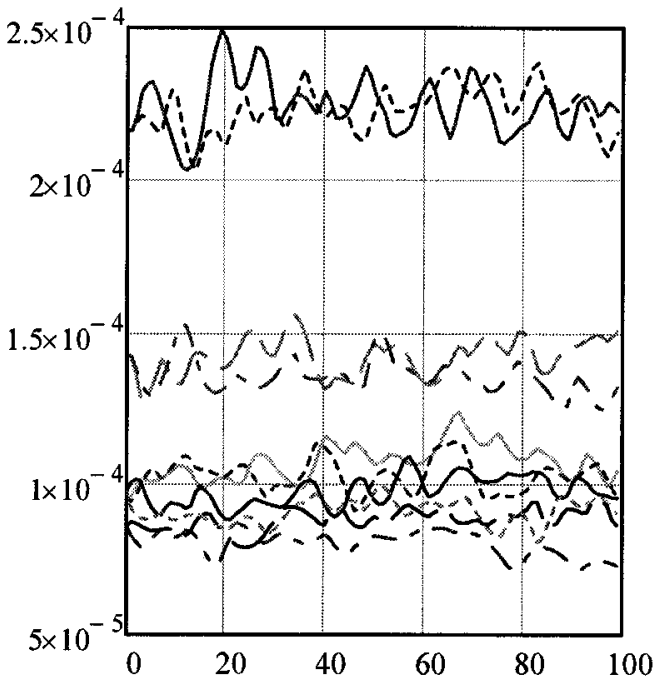

The field variable is $-\ln (\tau)$ so we must therefore take $-\ln$ of the data.

leftlnT := $\overrightarrow{-\ln (\operatorname{left} \tau)}$

rightln $\tau:=-\overrightarrow{\ln (\text { right } \tau)}$ 


\section{1-Dimensional Tomography}

The one-dimensional tomography is performed using a three-point Abel inversion method. The algorithm used was developed by Cameron J. Dasch (Dasch, Cameron J. "One-dimensional tomography: a comparison of Abel, onion-peeling, and filtered backprojection methods." Applied Optics. Vol 31, No. 8. 10 March 1992).

Enter the interval between radial points: gridint := int gridint $=0.1$

The number of data points:

$M:=\operatorname{rows}(\operatorname{left} \ln \tau)$

$\mathrm{M}=100$

Deconvolution operator follows. Matrix " $D$ " provides the linear operator coefficients, independent of $r$, that are used to deconvolve the input data.

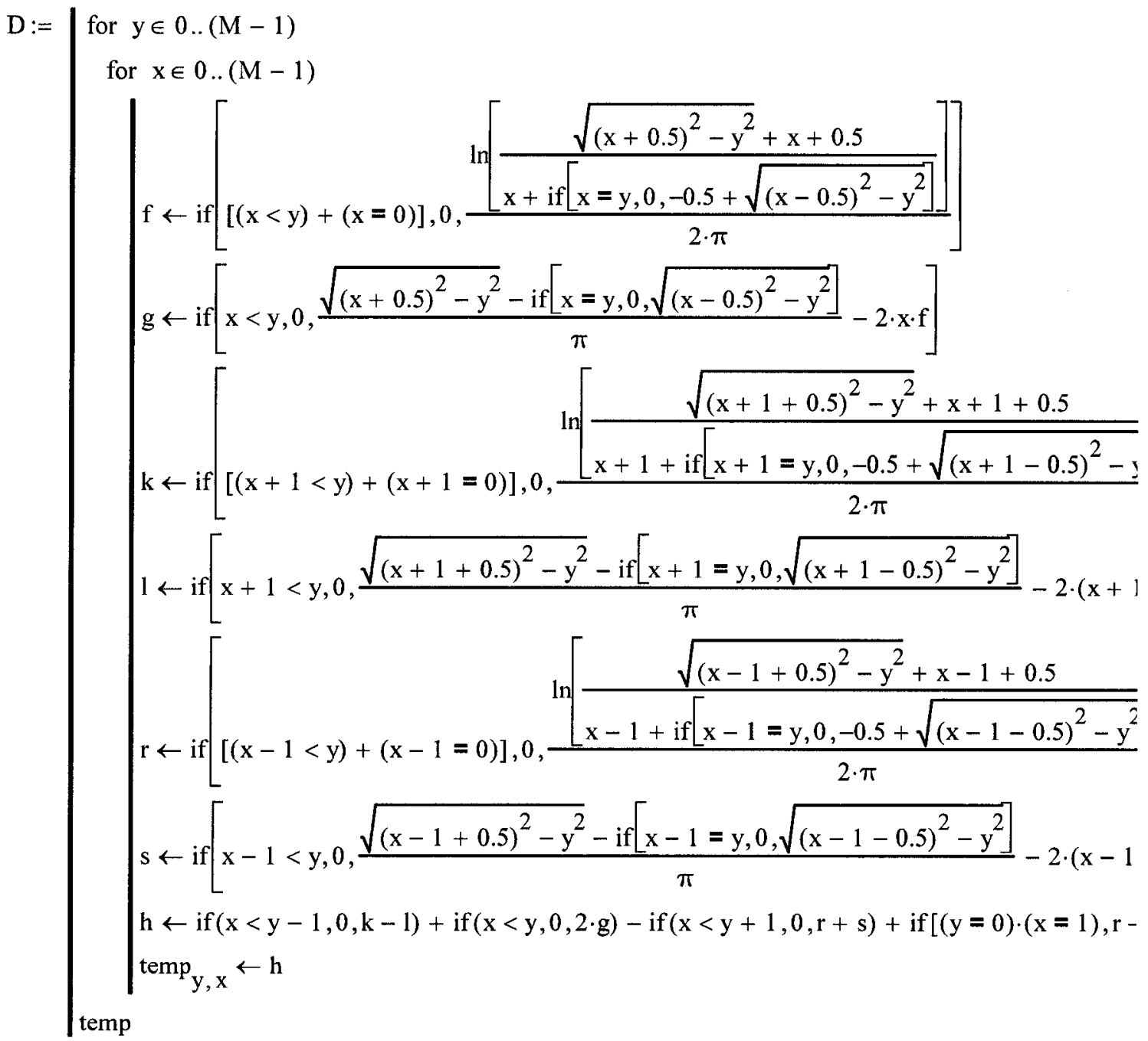

$\operatorname{rows}(D)=100 \quad \operatorname{cols}(D)=100 j$ 
Deconvolved Results: the input projection data 'a', at a spacing $r$ deconvolves to give $b$, the field distribution. Each element of $b$ contains a field distribution ( $a$ different matrix.)

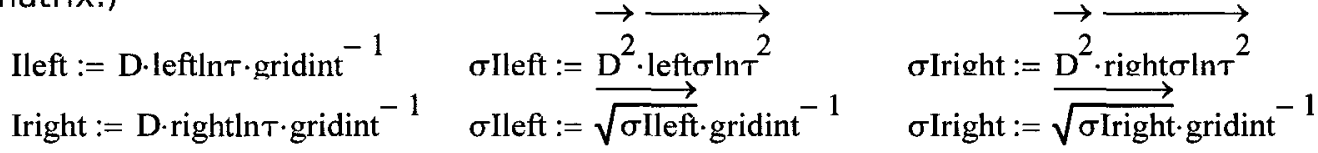

$$
\begin{aligned}
& \operatorname{rows}(\text { Ileft })=100 \quad \mathrm{i}:=0 . . \operatorname{rows}(\text { Ileft })-1 \\
& \text { Iav }:=\frac{\text { Ileft }+ \text { Iright }}{2} \quad \sigma_{\mathrm{av}}:=\frac{\sigma \text { Ileft }+\sigma \text { Iright }}{2}
\end{aligned}
$$

Inverted $\mathrm{dl} / \mathrm{dr}$ data, average of left and right (per $\mathrm{mm}$ )

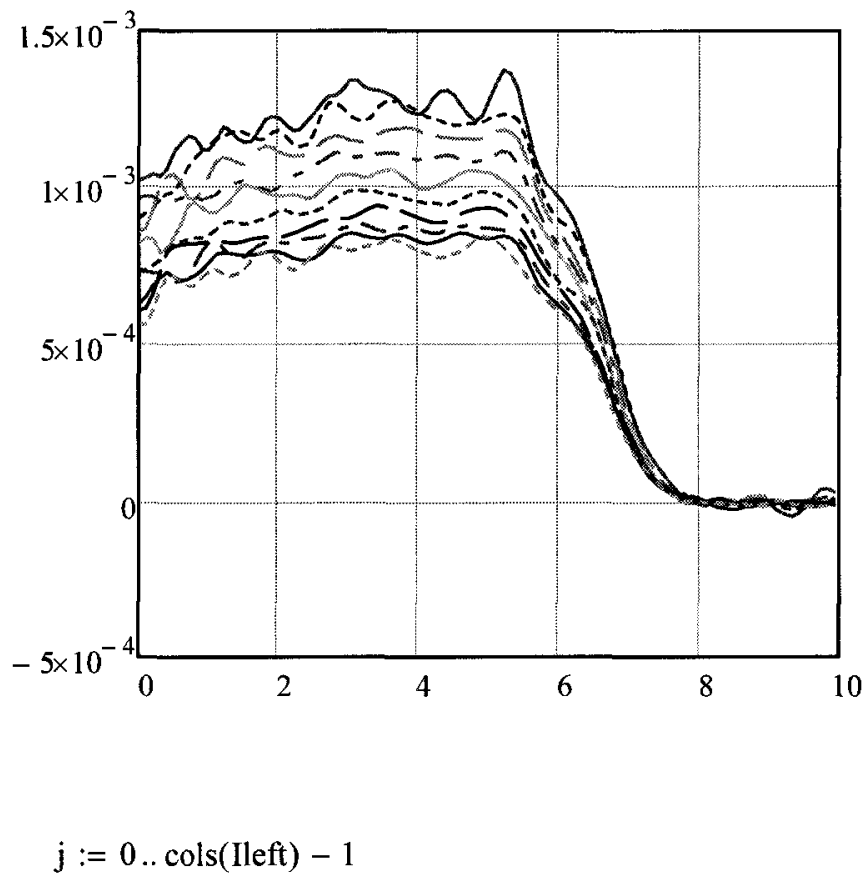

These limits define the radial limits to average within: Limit1 $:=0 \quad$ Limit2 $:=5$

$$
\begin{aligned}
& \text { round }\left({\text { Limit2. } \left.\text { gridint }^{-1}\right)}^{-1}\right. \\
& \sum^{\left(\operatorname{Iav}_{i, j} \cdot 2 \pi \cdot \operatorname{gridint}^{2} \cdot i\right)} \\
& \mathrm{K}_{\mathrm{ext}}:=\frac{\mathrm{i}=\text { round }_{\mathrm{j}}\left(\text { Limitl-gridint }^{-1}\right)}{\pi \cdot\left[\left(\text { round }\left(\text { Limit2 gridint }^{-1}\right) \cdot \text { gridint }^{2}-\left(\text { round }\left(\text { Limit1 gridint }^{-1}\right) \cdot \text { gridint }^{2}\right]\right.\right.}
\end{aligned}
$$




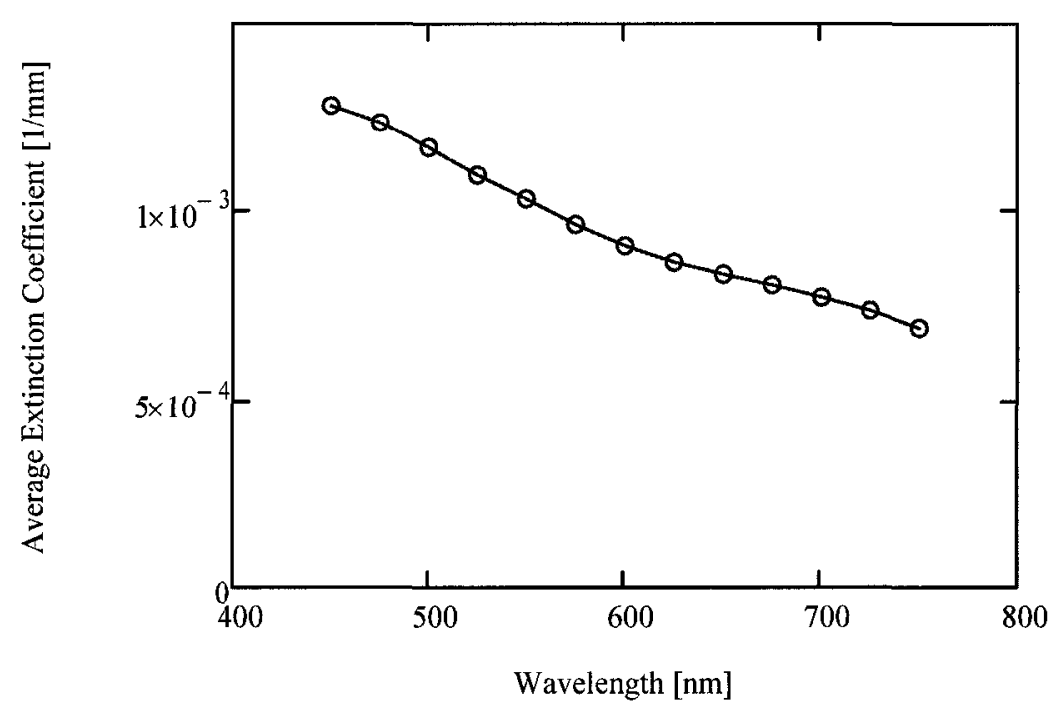

Propagating uncertainty in $95 \%$ confidence limit form for output:

$$
\begin{aligned}
& \text { round }\left(\text { Limit2. gridint }^{-1}\right) \\
& \sum^{2}\left(\sigma_{a_{i}, j} \cdot 2 \pi \cdot \text { gridint }^{2} \cdot \mathrm{i}\right)
\end{aligned}
$$

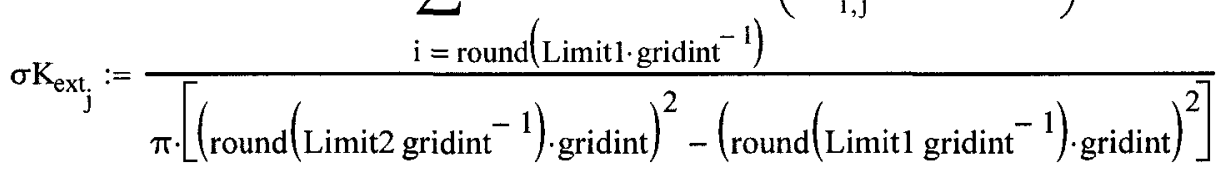

$$
\begin{aligned}
& \operatorname{errK}_{\mathrm{ext}}:=2.045 \cdot \frac{\sigma \mathrm{K}_{\mathrm{ext}}}{\sqrt{30}}
\end{aligned}
$$

Finally, output text files with the results, for use in later sheets.

$$
\text { output : }=\left\{\begin{array}{l}
\text { WRITEPRN }\left(\text { output_file("Kext"), augment }\left(\lambda, \mathrm{K}_{\mathrm{ext}}, \frac{\text { errK }_{\mathrm{ext}}}{\mathrm{K}_{\mathrm{ext}}}\right)\right) \text { if write_bool = } 1 \\
\text { WRITEPRN(output_file("Intau"), augment(radius, Intau_write) ) if write_bool = } 1 \\
0 \text { otherwise }
\end{array}\right.
$$




\section{Appendix C}

\section{Correlations in the Results}

An attempt was made at correlating the $E(m)$ results of this work with a series of parameters that varied among the tested conditions. Included among these parameters are those pertaining to the physical measurements of structure and concentration $\left(D_{f}, k_{g}, d_{p}, N, R_{g}, f_{v}\right)$, indirect quantities related to experimental operating parameters (exhaust stream residence time, characteristic aggregation time), and with other experimental parameters (wavelength, dilution ratio). Data were limited to four wavelengths, evenly spaced over the range tested $(450 \mathrm{~nm}, 600 \mathrm{~nm}, 750 \mathrm{~nm}$, and $900 \mathrm{~nm})$. A note of caution is warranted when interpreting the data at $900 \mathrm{~nm}$, as the uncertainty limits are considerably wider at such high wavelengths, as seen in Figure 4.13. Only values calculated using the self-preserving distribution were considered. 


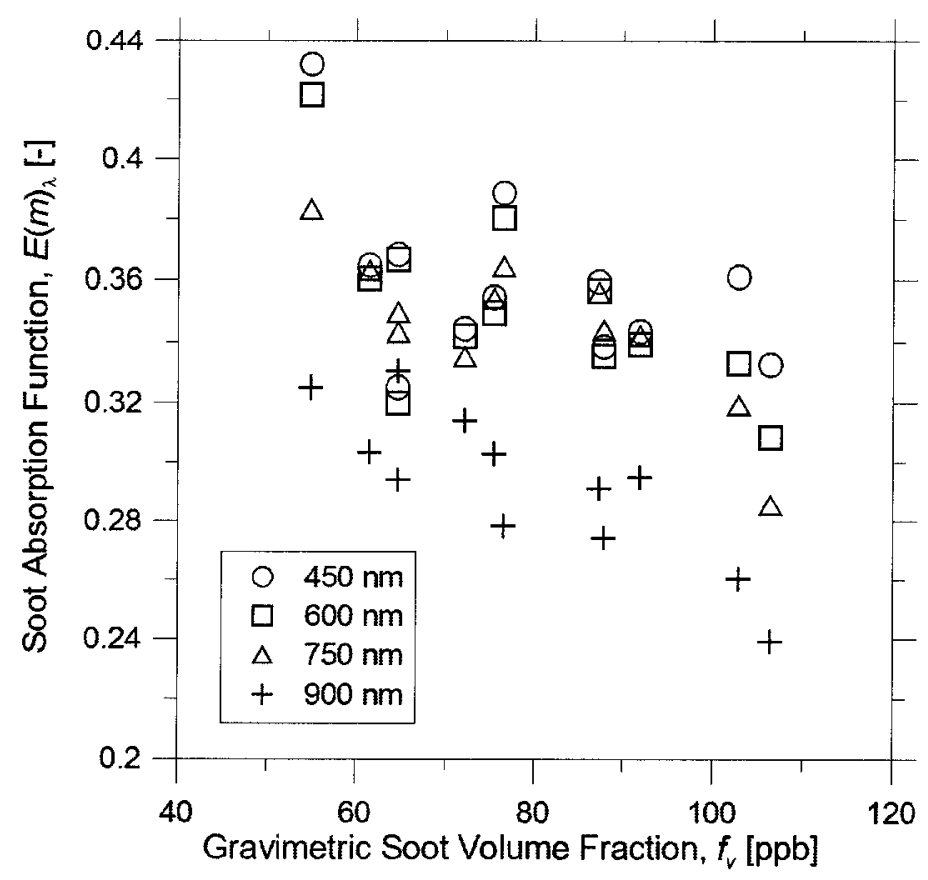

Figure C.1: Plot of $E(m)$ results versus gravimetric soot volume fraction, $f_{v}$

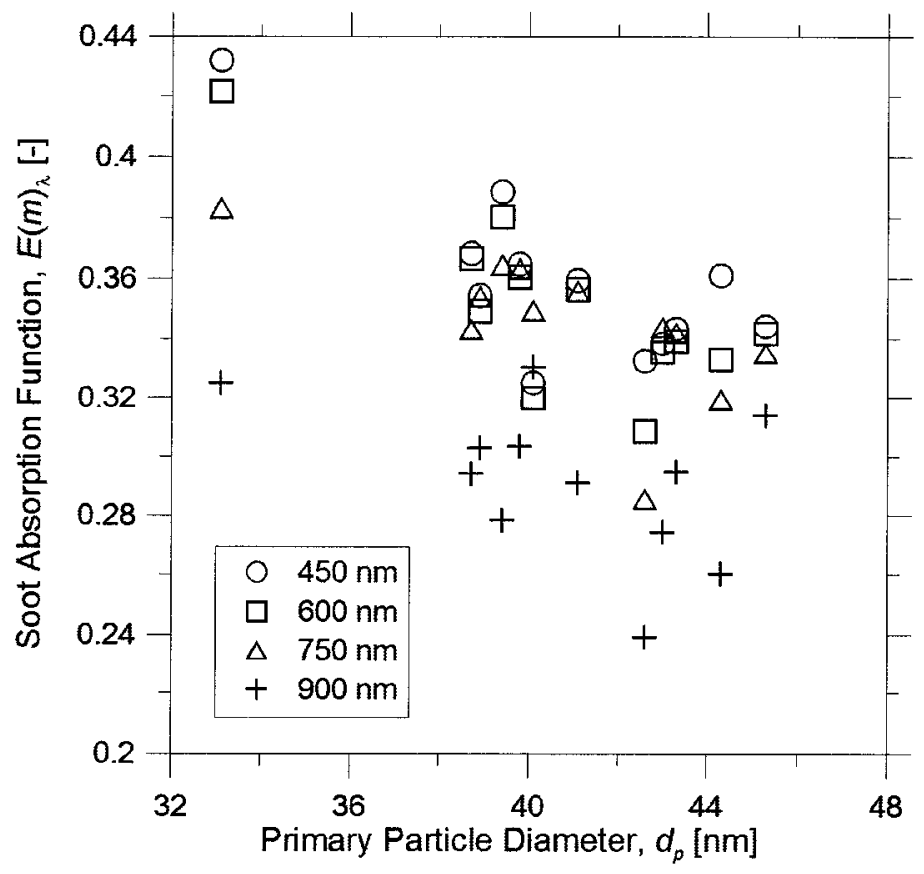

Figure C.2: Plot of $E(m)$ results versus primary particle diameter, $d_{p}$ 


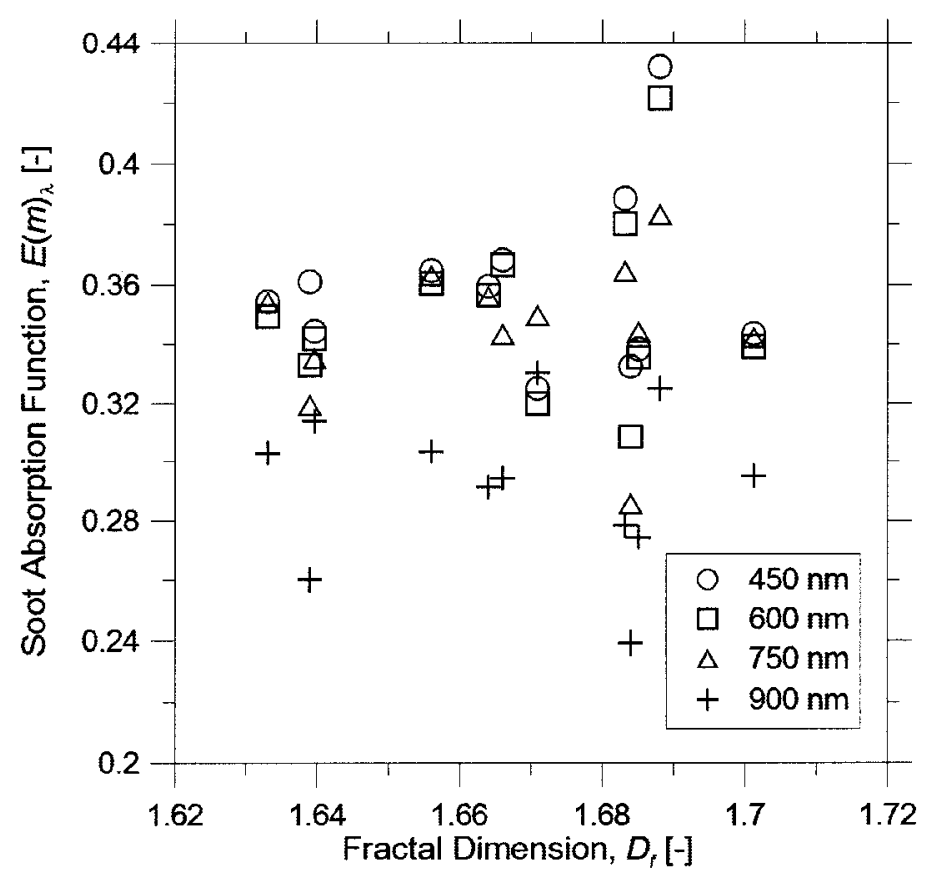

Figure C.3: Plot of $E(m)$ results versus fractal dimension, $D_{f}$

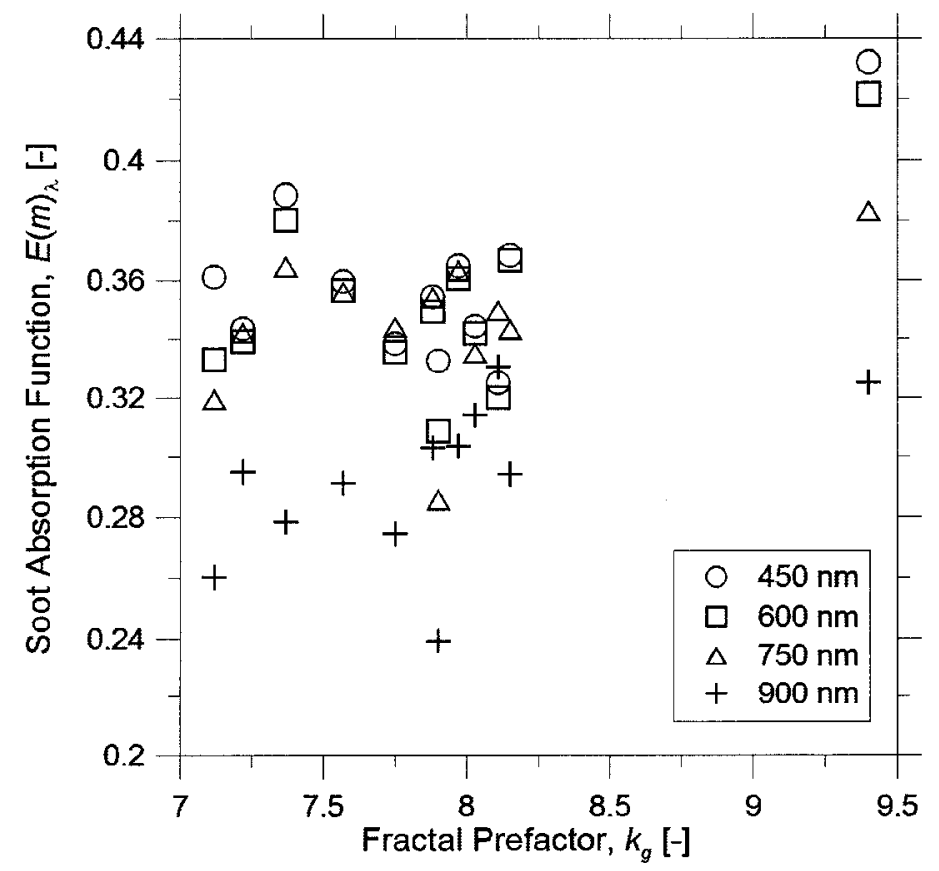

Figure C.4: Plot of $E(m)$ results versus fractal prefactor, $k_{g}$ 


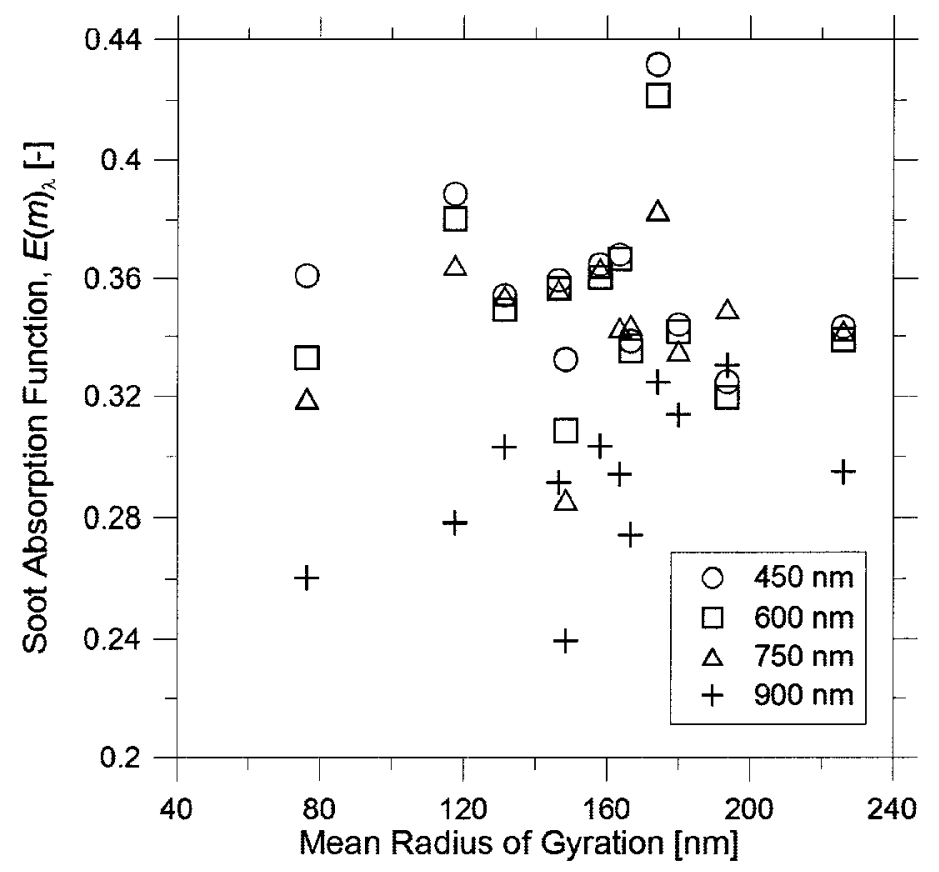

Figure C.5: Plot of $E(m)$ results versus radius of gyration, $R_{g}$

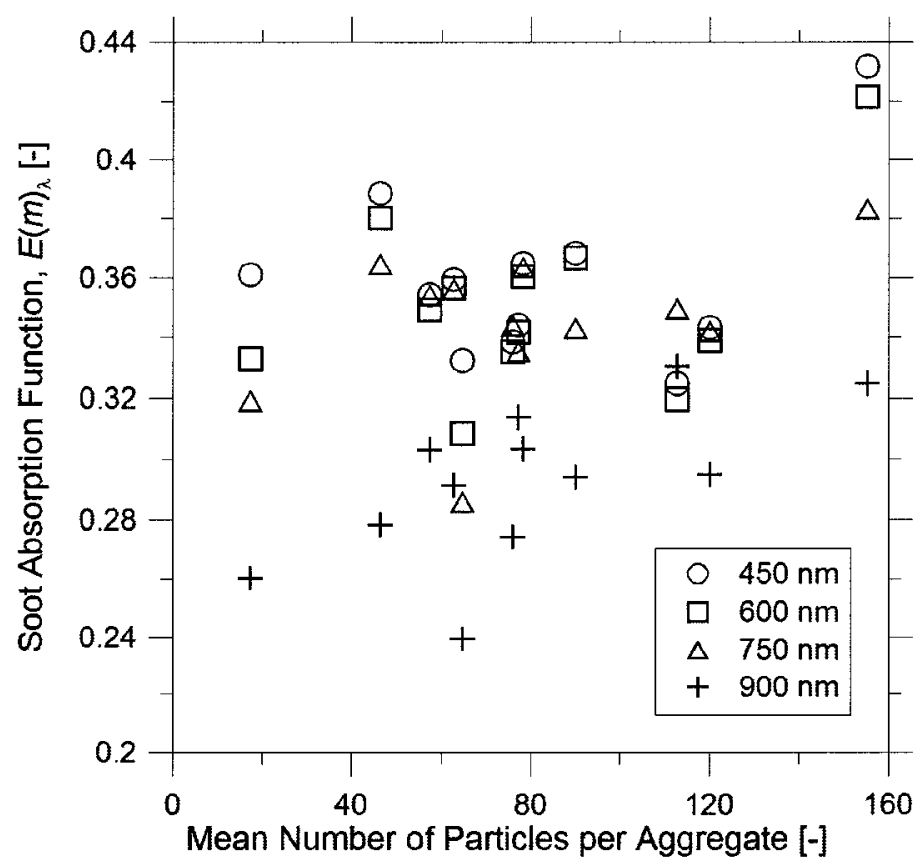

Figure C.6: Plot of $E(m)$ results versus mean value of $N$ 


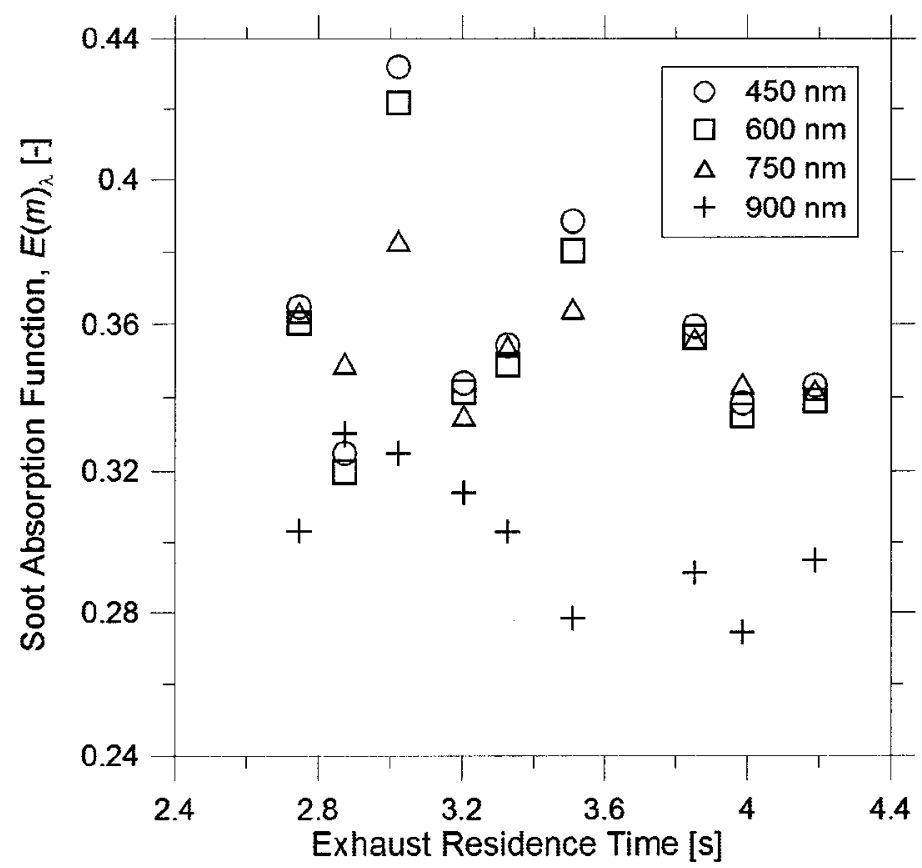

Figure C.7: Plot of $E(m)$ results versus exhaust residence time

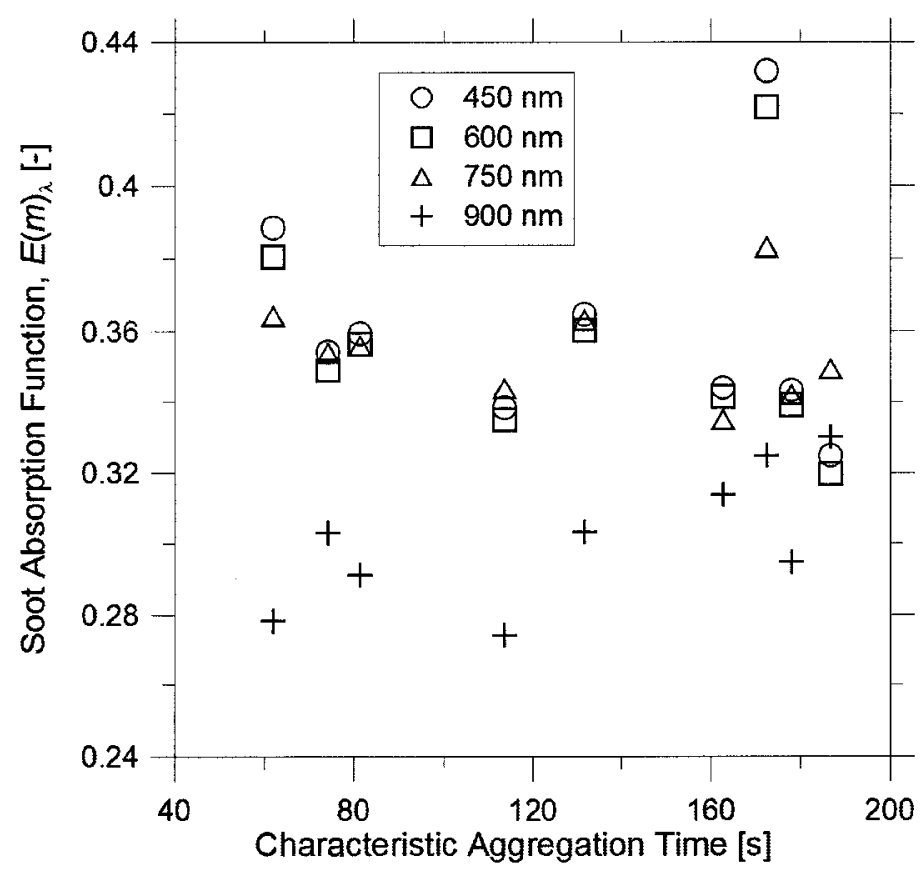

Figure C.8: Plot of $E(m)$ results versus characteristic aggregation time, $t_{c}$ 


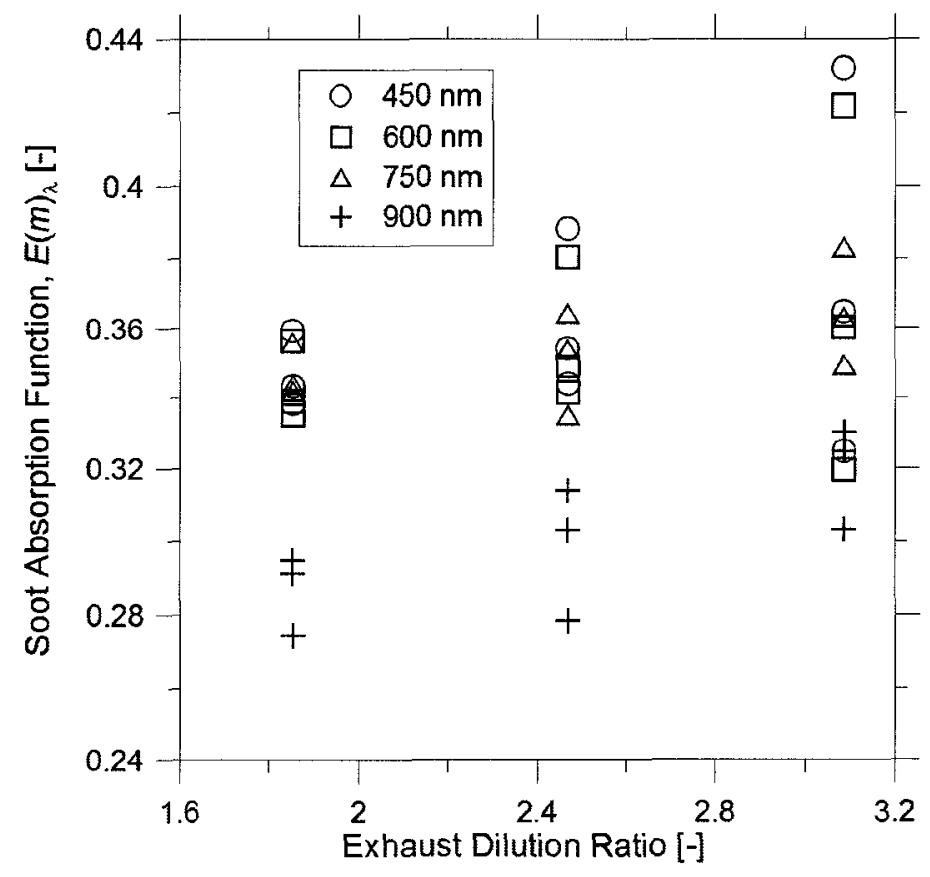

Figure C.9: Plot of $E(m)$ results versus dilution ratio, $D R$ 


\section{Appendix D}

\section{Correlations in Optical and Fuel}

\section{Properties}

Many studies have reported no apparent relationship between the fuel being burned and the optical properties of the resulting soot. Nevertheless, an attempt was made here to find correlations, using the results of the present work as well as the data from the studies shown in Figure 5.5. These data represent a broad spectrum of fuels, although they were obtained by differing measurement methods. With that caveat in mind, the following figures show the soot absorption function $E(m)$ plotted against several fuel parameters. Two fixed wavelengths were considered, $450 \mathrm{~nm}$ and $633 \mathrm{~nm}$, where much data exists. In cases where data did not exist at these wavelengths, they were estimated by linear interpolation between the two closest values. Nothing noteworthy was found, strengthening the notion that soot optical properties are independent of fuel type. 


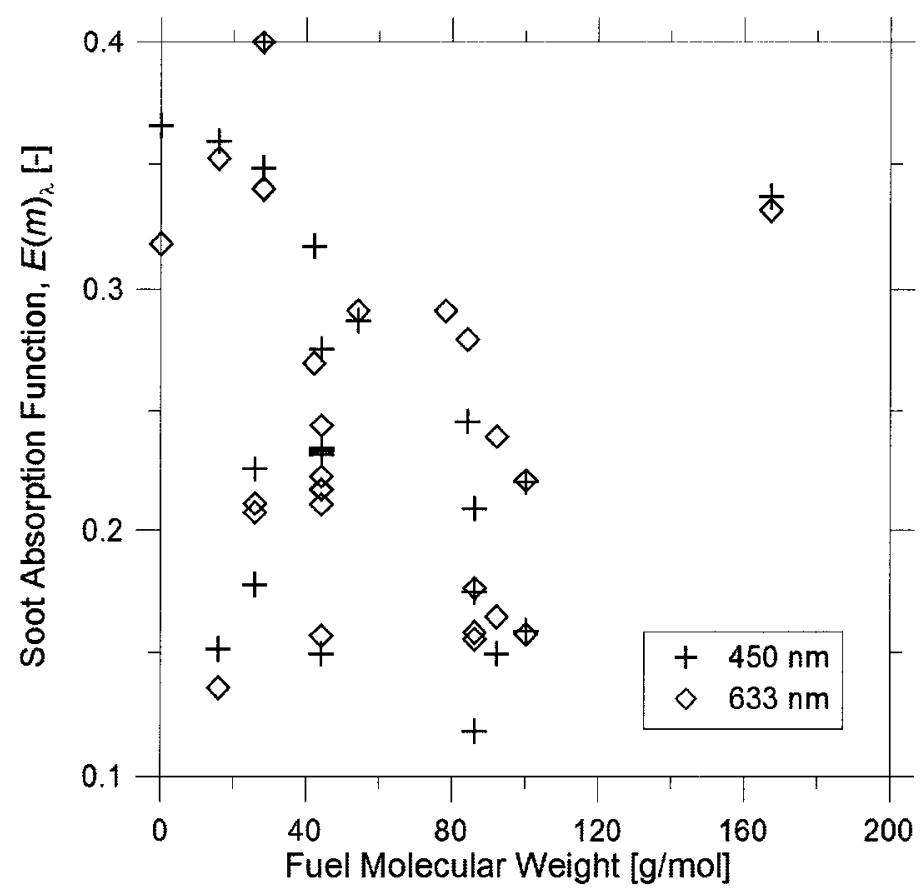

Figure D.1: Plot of $E(m)$ values versus fuel molecular weight

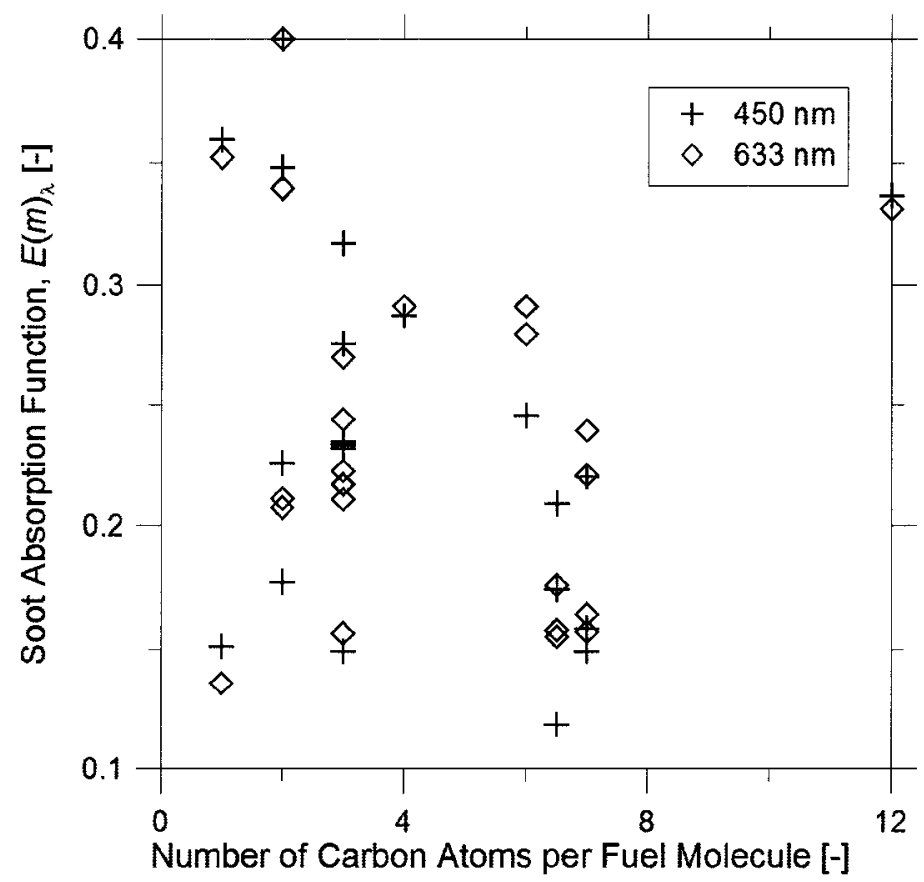

Figure D.2: Plot of $E(m)$ values versus number of carbon atoms per molecule of fuel 


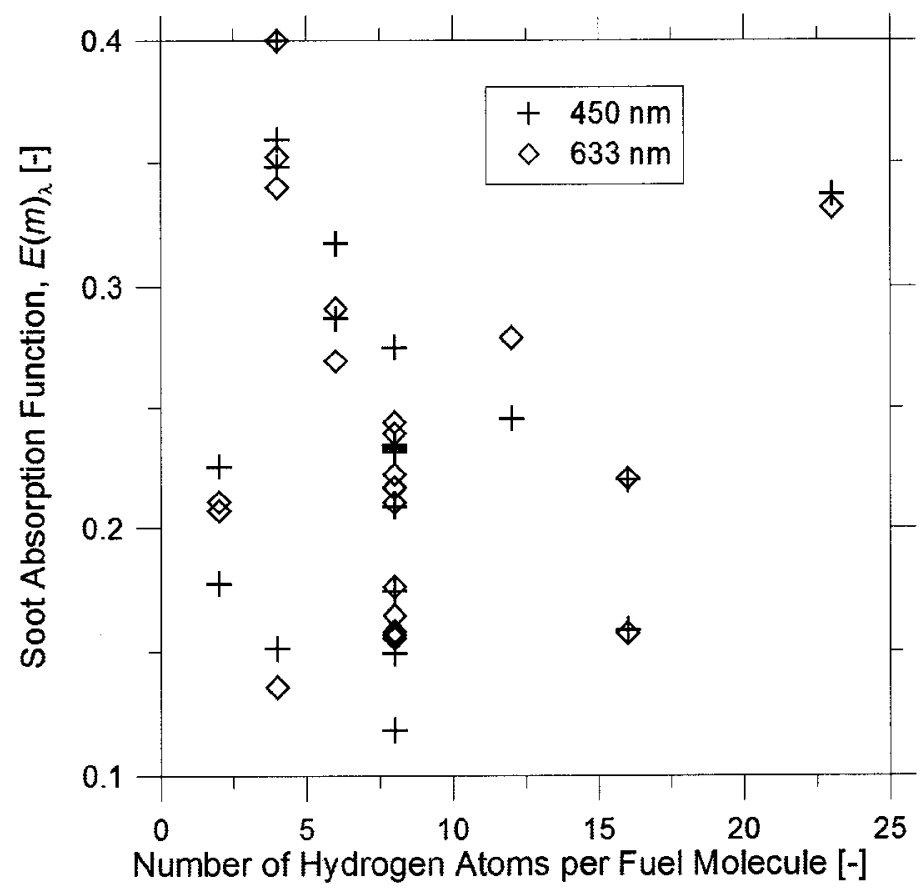

Figure D.3: Plot of $E(m)$ values versus number of hydrogen atoms per molecule of fuel

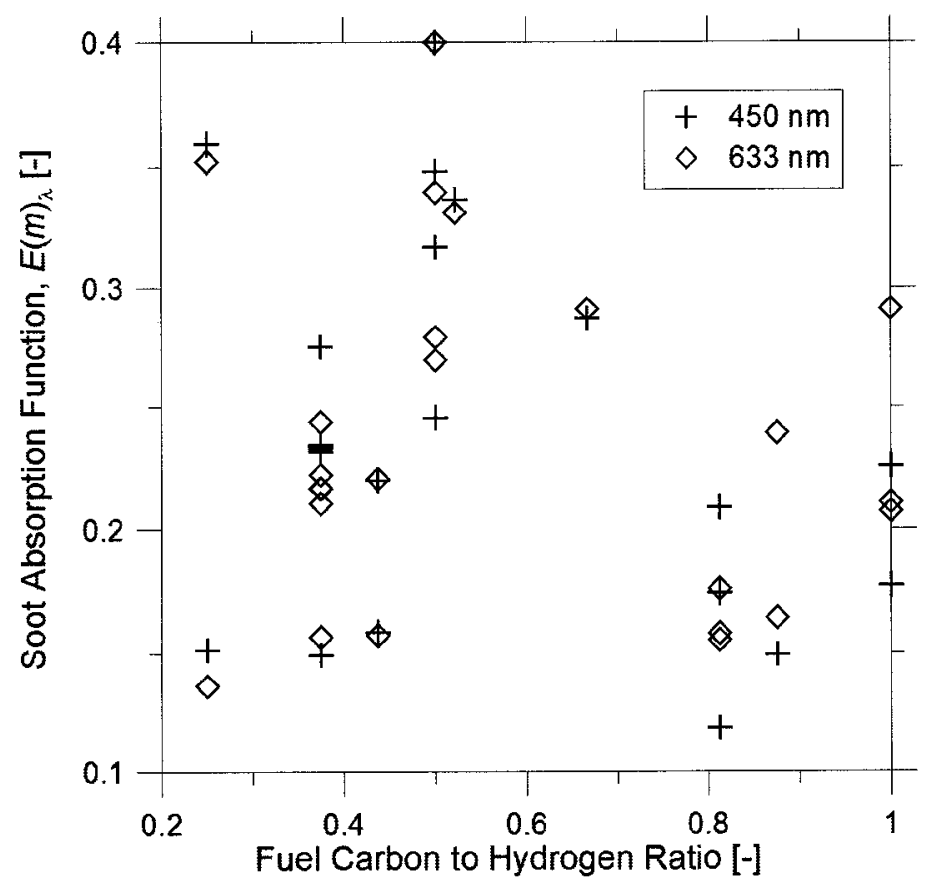

Figure D.4: Plot of $E(m)$ values versus a ratio of carbon to hydrogen 